\title{
Regularized Vortex Sheet Evolution in Three Dimensions
}

\author{
Thesis by \\ Mark A. Brady \\ In Partial Fulfillment of the Requirements \\ for the Degree of \\ Doctor of Philosophy
}

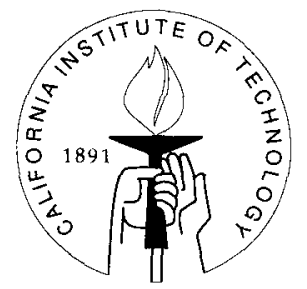

California Institute of Technology

Pasadena, California

2000

(Submitted June 15, 1999) 
(c) 2000

Mark A. Brady

All Rights Reserved 


\section{Acknowledgements}

Caltech would not have been an option for me were it not for the inspiring lectures of my undergraduate advisor, Professor Pozrikidis. I thank my current advisors, Professors Leonard and Pullin, for their guidance and support. They provided, I believe, an ideal combination of opinions, feedback, and distance. Grégoire Winckelmans also provided valuable insights of which I have taken advantage.

I am grateful for my wife Theresa's support and acceptance, both of life in Los Angeles and my frequent evening visits to the lab. I thank also friends and fellow students of Caltech - Chris Eckett, Ayhan Irfanoglu, Michel Jabbour, Ashish Misra, Piet Moeleker, Aurelius Prochazka, and Shaun Shariff - for countless conversations of levity and academics. I would also like to thank my parents, who have always wanted the best for me and watched over me.

This work was partially supported by NSF Grant CTS 9634222, ONR-N0001494-1-0793, and DE-AC03-98EE50506. 


\section{Abstract}

A computational method is presented to follow the evolution of regularized threedimensional (3D) vortex sheets through an otherwise irrotational, inviscid, constantdensity fluid. The sheet surface is represented by a triangulated mesh with interpolating functions locally defined inside each triangle. $C^{1}$ continuity is maintained between triangles via combinations of cubic Bézier triangular interpolants. The self-induced sheet motion generally results in a highly deformed surface which is adaptively refined as needed to capture regions of increasing curvature and to avoid severe Lagrangian deformation. Automatic mesh refinement is implemented with an advancing front technique. Sheet motion is regularized by adding a length scale cut-off to the BiotSavart kernel. Velocity evaluation takes less time than the standard $O\left(N^{2}\right)$ scaling, due to utilization of multi-pole expansions of the kernel. Zero, singly, and doubly periodic vortex sheets are simulated, modeling vortex rings, vortex/jet combinations and standard shear layers. Comparisons with previous two-dimensional (2D) results are favorable and 3D simulations are presented. The perturbed 3D planar shear layer is simulated and compared with a similar experiment revealing qualitatively similar results and agreement on the mechanism by which streamwise vorticity is created. We find the ratio of spanwise to streamwise vorticity to vary between 7 and 9 during early stages of roll-up.

A new technique for estimating the curvature singularity time of true vortex sheets (i.e., non-regularized motion) is presented. The motion and singularity time of a planar, doubly periodic sheet, evolving from a 3D normal mode perturbation, is found to reduce to that of a well known singly periodic (and only two-dimensional) problem, an unexpected extension of Moore's [38] non-linear analysis for 2D vortex sheets. 


\section{Contents}

Acknowledgements

Abstract

List of Figures

List of Tables

1 Introduction

1.1 Ill-Posedness of Vortex Sheets . . . . . . . . . . . . . . . . 2

1.2 Regularization of Vortex Sheet Motion . . . . . . . . . . . 3

1.3 Suitability of the Smooth Vortex Sheet Model . . . . . . . . . . 4

1.4 3D Regularized Vortex Sheets ... . . . . . . . . . . . . 6

1.5 Overview ............................. 7

2 Theoretical Development $\quad 8$

2.1 Parametric Surface . . . . . . . . . . . . . . . 8

2.2 The Velocity Field . . . . . . . . . . . . . . . . 10

2.3 Vortex Sheet Motion .................. 11

2.4 Surface Types: SWP0, SWP1 and SWP2 . . . . . . . . . 15

2.5 Invariants . . . . . . . . . . . . . . . . 17

3 Computational Development $\quad 19$

3.1 Surface Interpolation . . . . . . . . . . . . . . . . . . . 19

3.2 Integration . . . . . . . . . . . . . . . . . 21

3.3 Parallelism . . . . . . . . . . . . . . . . 22

3.4 SWP1 Summation Approximation . . . . . . . . . . . . . 23

3.5 Trigger for Mesh Refinement . . . . . . . . . . . . . . . 23 
3.6 Pseudocode . . . . . . . . . . . . . . . . . . . . . 24

3.7 Computational Hardware . . . . . . . . . . . . . . . . . . 24

4 Mesh Generation $\quad 27$

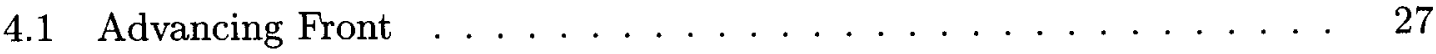

4.2 Density Function . . . . . . . . . . . . . . . . 27

4.3 Triangle Size Scaling . . . . . . . . . . . . . . . . 28

4.4 Stretching Directions . . . . . . . . . . . . . . 29

4.5 Boundary Node Placement . . . . . . . . . . . . . . . . . . 29

4.6 Interior Ideal Node Placement $\ldots \ldots \ldots$

4.7 Implementation . . . . . . . . . . . . . . . 30

4.8 Mesh Post-Processing . . . . . . . . . . . . . . . . . . . 31

5 Improved Velocity Evaluation $\quad 35$

5.1 Introduction . . . . . . . . . . . . . . . 35

5.2 Velocity Evaluation . . . . . . . . . . . . . . . 36

5.3 Scaling Arguments . . . . . . . . . . . . . . . . . . 37

5.4 Expansion, Error Bound, and Critical Distance $\ldots \ldots \ldots . \ldots 39$

6 Periodic Velocity Evaluation $\quad 44$

6.1 Introduction . . . . . . . . . . . . . . . . . 44

6.2 Example . . . . . . . . . . . . . . . . . . 45

6.3 Implementation . . . . . . . . . . . . . . . 47

7 Performance $r$

7.1 Example Initial Condition . . . . . . . . . . . . . 50

7.2 Run-time Parameters . . . . . . . . . . . . . . . . 51

7.3 Remeshing Frequency . . . . . . . . . . . . . . . . . 51

7.4 Convergence Analysis . . . . . . . . . . . . . . . . . 52

7.5 Comparison with Exact Velocities . . . . . . . . . . . . 54

$7.5 .1 \quad$ SWP1 ............................. 54

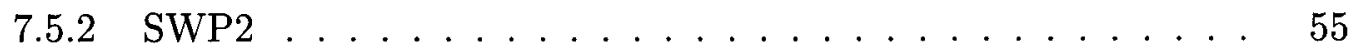


vii

7.6 Comparison with 2D Results . . . . . . . . . . . . 55

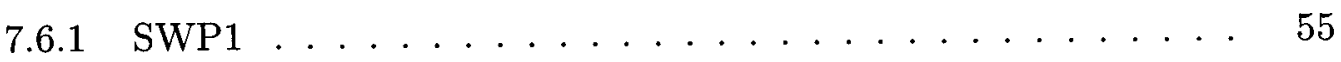

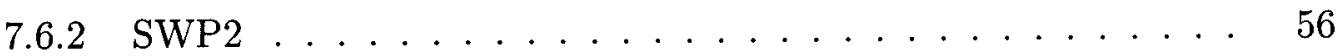

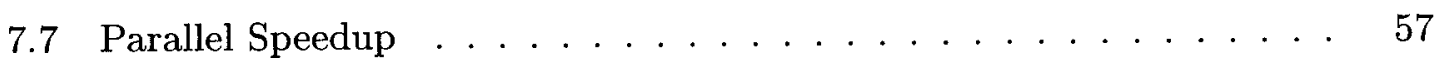

8 Examples $\quad 71$

8.1 Jet $(\mathrm{SWP} 1) \ldots \ldots \ldots 71$

8.2 Vortex Rings, Co-axial $(\mathrm{SWPO} \times 2) \ldots \ldots . . \ldots 72$

8.3 Vortex Rings, Offset $(\mathrm{SWP0} \times 2) \ldots \ldots . \ldots 73$

8.4 Vortex Rings, Interlocked $(\mathrm{SWP0} \times 2) \ldots . . . . . . . . .74$

9 Shear Layer Application $\quad \mathbf{8 5}$

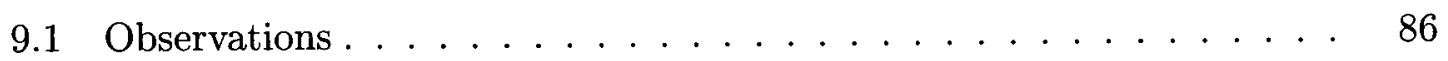

9.2 Other Experiments . . . . . . . . . . . . . . . 87

10 Vortex Sheet Critical Time $\quad 100$

10.1 Background ...................... . . . 100

10.2 Finite-time Singularity for 2D Vortex Sheets . . . . . . . . . . . 101

10.3 Numerical Estimation of the Critical Time, $t_{c}$. . . . . . . . . 102

10.3.1 2D evolution, $2 \mathrm{D}$ initial condition . . . . . . . . . 104

10.3.2 3D evolution, $2 \mathrm{D}$ initial condition . . . . . . . . . 105

10.3.3 3D evolution, 3D initial condition . . . . . . . . . 106

11 3D Normal Mode Perturbations 112

11.1 Introduction . . . . . . . . . . . . . . . . . 112

11.2 Vortex Sheet Evolution . . . . . . . . . . . . . . 112

11.3 Evolution of an Initially Cylindrical Vortex Sheet . . . . . . . . . 113

11.4 Finite-time Singularity for 3D Vortex Sheets . . . . . . . . . . . 116

12 Discussion $\quad 119$

12.1 Directions for Future Work . . . . . . . . . . . . . . 120 
viii

Bibliography

122

$\begin{array}{ll}\text { A Notation } & 128\end{array}$

B Invariants $\quad 131$

C Surface Interpolant Details $\quad 134$

$\begin{array}{ll}\text { D Regularizing the 2D, Periodic Kernel } & 136\end{array}$

E Evaluation of the Double Periodic Summations $\quad 138$ 


\section{List of Figures}

1.1 Comparison of vortex ring formation (reproduced from [42]). Experiment visualization above with dyed material line of fluid. Simulation below utilizing smooth kernel $(\sigma=0.2)$ depicting calculated material line. . . . . . . . . . . . . . . . . . . 5

2.1 Mapping from parameter to physical space. . . . . . . . . . . . 9

2.2 Vortex sheet dividing an ideal fluid. . . . . . . . . . . . . . 11

3.1 (a) Raw and (b) post-processed parameter and corresponding physical space for a torus. . . . . . . . . . . . . .

3.2 Demonstration via mesh skeletons of the need for mesh refinement. Lagrangian deformation of material elements in (a) reveals a loss of resolution with a fixed mesh (600 triangles). With the same initial condition, timely remeshing in (b) allows for an accurate representation of the sheet $(5000$ triangles $) \ldots \ldots \ldots \ldots \ldots$

4.1 Example ideal node placement and usage, active edge shown in red. In (a), an existing node (filled circles) in the front is close enough to the ideal position (open circle) to be substituted. In (b) there are no nearby existing nodes - a new node will be inserted at the ideal position

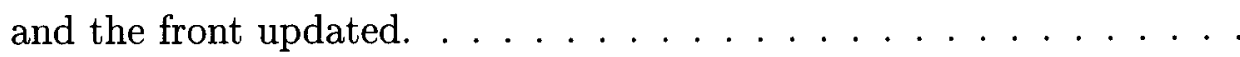

4.2 Advancing front in parameter space $\Sigma$ with $185,1000,2000$, and 2650 triangles. Note how in (d) the front has pinched itself closed, generating two child fronts. . . . . . . . . . . . . . . . . . . . 33

4.3 Dashed lines represent (a) swapped and (b) un-swappable edges. . . . 33 
4.4 The two black triangles conform nicely to the local surface topology. The edge in the valley is considered for swapping with the alternate red edge. The ECD of the black edge is approximately zero, as its midpoint is essentially on the surface, but the distance of the midpoint of the red edge to the surface is significantly more. Thus the black edge will not be swapped for the red.

5.1 An example triangulated sheet encased in a non-hierarchical subdivision of boxes, each with side length $L_{B} \ldots \ldots \ldots \ldots \ldots$

5.2 Duration of one time step (single processor) versus the number of triangles, $N . O\left(N^{2}\right)$ method in red; $O\left(N^{3 / 2}\right)$ method in green and blue. Solid lines have slopes $2.0,1.55$ and 1.57 respectively. With $E_{T O L}=10^{-3}$, speed is increased by a factor of 10 near $N=20,000$. .

5.3 CPU time per time step plotted against the number of current triangles in the mesh $(N)$ for an example simulation (see Appendix 7.1). Direct $O\left(N^{2}\right)$ method in green, $O\left(N^{3 / 2}\right)$ method in red. Time steps in the simulation implicitly travel from left to right, $N$ increasing as the sheet evolves. Numbers next to lines specify the slope. . . . . . . . . . . . .

5.4 Patch of triangulated vortex sheet inside box with volume $V . \tilde{\boldsymbol{x}}$ is the box centroid and $\boldsymbol{x}^{\prime}$ is a point on the sheet surface. . . . . . . . .

5.5 Patch of triangulated vortex sheet inside box with volume $V$. Schematic of box-target interaction. . . . . . . . . . . . . . . . . . . .

5.6 PDF of scaled difference between $O\left(N^{3 / 2}\right)$ time step and an $O\left(N^{2}\right)$ time step. $E_{T O L}$ decreases with symbol darkness from $10^{-2}$ to $10^{-3}$ to $10^{-4}$. Average strain (error) drops roughly from $10^{-3}$ to $10^{-4}$ to $10^{-5}$.

6.1 Images about the origin of a doubly periodic function. Shaded regions are labelled: the (a) base period $P_{0}$, (b) first ring $P_{1}$, (c) first and all remaining rings $P_{1 \infty}$, (d) second and all remaining rings $P_{2 \infty}$. Black circles represent the accompanying lattice of period centered points, $\tilde{P}_{i}$. In (c) and (d) the shaded region and lattice extend without bound. 
7.1 Shaded mesh skeleton of the example configuration, a self-impinging jet (SWP0), with $\sigma=0.2$ relative to initial tube diameter: (a) $\mathrm{t}=0$, (b) $\mathrm{t}=1.1$, (c) $\mathrm{t}=2.2$, (d) $\mathrm{t}=3.0$. Contours depict slices through the surface. 58

7.2 Variation of invariants with resolution $R . R=10$ (red), 20 (green), 30 (blue), 40 (cyan). Initial condition from $\$ 7.1 \ldots \ldots \ldots \ldots \ldots$

7.3 Variation of invariants with error tolerance $E_{T O L}: E_{T O L}=0.001$ (red), 0.0001 (green), 0 (blue). Initial condition from $\S 7.1 \ldots \ldots \ldots$. . . .

7.4 Variation of invariants with the number of Gauss integration points: 3 points (red), 4 (green), 6 (blue). Initial condition from $\$ 7.1$. . . . .

7.5 Variation of invariants with the time step $\Delta t: \Delta t=0.2$ (red), 0.1 (green), 0.05 (blue). Initial condition from $\$ 7.1 \ldots \ldots \ldots \ldots$

7.6 Variation of invariants with different mesh refinement triggering. Example simulation (from $\S 7.1$ ) with parameters chosen in $\$ 7.2$. No remeshing (red), moderate remeshing with $t_{L R}=2, t_{N C}=20 \%$ (green), frequent remeshing with $t_{L R}=1.5, t_{N C}=10 \%$ (blue) . . . . .

7.7 Maximum error $\mathrm{E}$ versus the number of triangles for each component of $\mathbf{X}$ (hollow points), $\mathbf{X}_{\xi}$ (filled), and $\mathbf{X}_{\eta}$ (filled). Dashed line has slope $m=-1.5$. Solid line has slope $m=-1.0$.

7.8 Spatial order of convergence with a regular grid and the example configuration as a shaded contour plot over the parameter space, $\Sigma$. Dots represent vertices for the lowest resolution case $(R=5)$. (a) 4 point Gaussian quadrature, (b) 6 point Gaussian quadrature. . . . . . . . .

7.9 Qualitative spatial convergence demonstrated with a slice through the sheet surface at constant $z$ at $t=2.6, R=10,20,40 \ldots \ldots \ldots$

7.10 Shaded mesh skeleton and contour slices with reduced kernel smoothing parameter, $\sigma=0.1$ relative to initial diameter. Contours are slices through the surface. . . . . . . . . . . . . . . . 
7.11 Comparison of numerical method (points) with exact velocities (lines) from the modified Biot-Savart kernel Eq. 2.22 with $\sigma=0.25,0.1$, (filled circles) and 0.01 (open circles). Curves steepen with decreasing $\sigma$. Note the dramatic difference in accuracy for $\sigma=0.01$ in (a) 500 and (b) 8000 triangles. . . . . . . . . . . . . . . . . . .

7.12 Sampled velocity profile above the regularized planar vortex sheet (red) and the exact solution from Eq. 7.9 (black). Samples taken from a uniform 25 by 25 grid over the sheet. Absolute error of 0.005 at a height of $5 \sigma \ldots \ldots \ldots \ldots \ldots \ldots \ldots \ldots \ldots$

7.13 Comparison of current 3D method (solid lines representing surface, projected into $y-z$ plane) with 2D cylindrical vortex sheet (dashed lines). (a) $\sigma=0.1$ (b) $\sigma=0.05 \ldots \ldots \ldots \ldots \ldots$

7.14 Comparison of current 3D method (SWP2 in red), projected into the the $(x-z)$ plane with 2D singly periodic vortex sheet (black line). $\sigma=0.05$, initial perturbation amplitude $\epsilon=0.05 . \ldots \ldots \ldots$

7.15 Program speed-up $S$ relative to a single processor execution at each time step of the example simulation (from $§ 7.1$ ). Two processors (red), three (green), and four (blue). Only the velocity evaluation routine is parallelized; hence the speed-up is not perfect.

7.16 Cumulative program speed-up $S$ incorporating both four processors and the multi-pole velocity evaluation technique at each time step of the example simulation (from $§ 7.1$ ).

8.1 SWP1 vortex sheet with strength distribution simulating a hollow-core jet with initial $1^{\text {st }}$ mode axial perturbation, $\sigma=0.2$ (relative to initial average diameter). One period calculated, two shown with second in cut-away. Shaded mesh skeleton: (a) $\mathrm{t}=0$; (b) $\mathrm{t}=0.2 ;$ (c) $\mathrm{t}=0.4$; (d) $\mathrm{t}=0.6$; (e) zoom of roll-up region in (d); (f) actual smooth surface representation of $(\mathrm{e}) \ldots \ldots \ldots \ldots \ldots \ldots$ 
xiii

8.2 Shaded mesh skeleton of SWP1 vortex sheet with strength distribution simulating a hollow-core jet at $t=0.12$. Initial $1^{\text {st }}$ mode axial perturbation. One period calculated, two shown with second in cut-away. (a)

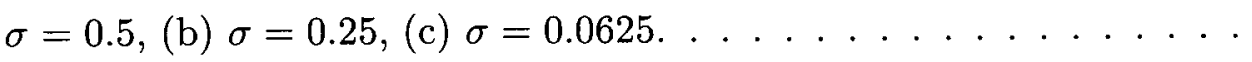

8.3 Slice through the vortex ring model, depicting instantaneous $(t=0)$ velocity field in a frame moving with the vortex ring. The axes are radial distance $r$ from the center of the ring, and distance $x$ parallel to the impulse vector. Color contours represent the velocity magnitude, from zero (dark blue) to 1.8 times the propagation velocity (red); black lines are instantaneous streamlines; the white circle is the vortex sheet surface.

8.4 Shaded mesh skeleton of hollow-core vortex rings (SWP0) with in-line impulse vectors, $\sigma=0.2$ (relative to initial tube diameters). Ring to tube radius ratio is 4 . Right side is a vertical slice through the ring centers; (a) $\mathrm{t}=0$, (b) $\mathrm{t}=0.44$, (c) $\mathrm{t}=0.88$.

8.5 Shaded mesh skeleton of hollow-core vortex rings (SWP0) with offset impulse vectors, $\sigma=0.2$ (relative to initial tube diameters). Ring to tube radius ratio is 4 . Right side is a vertical slice through the ring centers; (a) $\mathrm{t}=0$, (b) $\mathrm{t}=0.44$, (c) $\mathrm{t}=0.88 \ldots \ldots \ldots$

8.6 Time slice corresponding to Fig. 8.5(c), colored by sheet strength $|\gamma|$. Vorticity has localized to thin bands on each surface. . . . . . . .

8.7 Mesh skeleton corresponding to Fig. 8.5(c), detailing triangulation. Note the high curvature fold developing in the bottom portion of the surface.

8.8 Shaded mesh skeleton of interlocked hollow-core vortex rings (SWP0) with perpendicular impulse vectors. $(\sigma=0.2$ relative to initial tube diameter; ring to tube radius ratio is 4.) Vertical ring impulse points left and into the page, while horizontal ring impulse points up. (a) $\mathrm{t}=0$, (b) $\mathrm{t}=0.28,(\mathrm{c}) \mathrm{t}=0.54,(\mathrm{~d})$ zoom of pinched region in (c). . . . 
xiv

9.1 Roll-up of a perturbed shear layer (SWP2). One period calculated, three shown shaded, one shown with two vertical slices; (a) $t=0$, (b) $t=1.0$, (c) $t=1.8$; (d) only triangles with vorticity magnitude over a threshold are plotted, revealing the connectivity of the streamwise vorticity. Vortex lines follow the arrows: spanwise (blue), streamwise

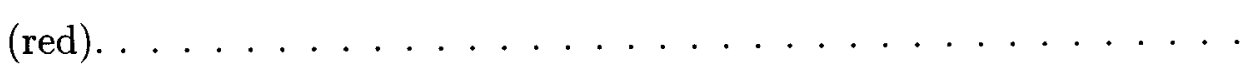

9.2 Different viewing angle of Fig. 9.1(c), revealing sheet strength $|\gamma|$ and vortex lines. Vorticity has localized to streamwise loops (red) and spanwise rollers (red, hidden), leaving a majority of the sheet surface with little vorticity (blue). . . . . . . . . . . . . . . .

9.3 Approximate circulation (a) held in the spanwise ( 0 ) and streamwise $(\diamond)$ vortices as a function of time; (b) ratio of the spanwise to streamwise circulations. . . . . . . . . . . . . . . .

9.4 Variation of the Kelvin-Helmholtz roll-up with span at the final computed time, $t=1.8$. (a) $y=-0.125$, (b) $y=0$. In (b), the developing streamwise vorticity is not uniformly parallel to the $x$ axis, as implied by the spanwise deviation of the fold from the planar cut. . . . . . . .

9.5 Variation of the Kelvin-Helmholtz roll-up with streamwise location at the final computed time, $t=1.8$. (a) $x=-0.25$, (b) $x=-0.125$. . .

9.6 Close-up of spiral region in Fig. 9.5(c), shaded with sheet strength magnitude, $|\boldsymbol{\gamma}|$, from maximum in red to minimum in blue. Head on (a), and at a slight angle (b).

9.7 Similarity of perturbed shear layer evolution: (a) snapshot of the slowspeed side of a spatially and temporally developing shear layer (experiment by Lasheras \& Choi [30]), (b) shear layer interface of doubly periodic calculation as described in $\S 9.1$. See text for conditions of the

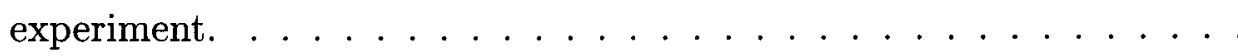

10.1 Sketch of a planar vortex sheet with velocity jump $U . \ldots . . . . .$. 
10.2 Example $\left(t, \kappa_{\mathrm{m}}\right)$ data (dots) from 2D evolution defined by Eq. D.3 and sheet parameters $\epsilon=0.01, \sigma=.05$ (see Eq. 10.9). Also four different curve fits (lines from left to right following arrow) of Eq. 10.8 with $t_{1}=0.75 t_{2}, 0.8 t_{2}, 0.85 t_{2}$ and $0.9 t_{2}$. In this case $t_{2}=0.84 \ldots \ldots 104$

10.3 Example roll-up of a 2D, periodic vortex sheet $(\epsilon=.01$ and $\sigma=.05)$ modeling a shear layer, subject to evolution Eq. D.3. Three snapshots represent the times (a) $t=0$, (b) $t=t_{c, \sigma}$, and (c) $t>t_{c, \sigma} \ldots \ldots \ldots$ 106

10.4 Quadratic fits used to extrapolate $t_{c, \sigma}$ to $\sigma=0$ with data from sheets driven by (a) Eq. D.2 and (b) Eq. D.3, both with initial condition given by Eq. 10.9 and $\epsilon=0.01$. Both approach $t_{c} \approx 0.39$ as $\sigma \rightarrow 0$, independent of the temporal window used ( $t_{1}$ is a larger fraction of $t_{2}$ following arrow $) \ldots \ldots \ldots \ldots \ldots \ldots$

10.5 Good agreement with techniques used to evaluate $t_{c}$ : data from [28] $(\bullet)$; extrapolated $t_{c}$ values from Eq. D.2 $(\square)$; extrapolated $t_{c}$ values from Eq. D.3 $(\diamond)$; theoretical prediction from Eq. 10.6 (line). . . . . . 108

10.6 One period ( $P_{0}$ from Fig. 6.1) of the doubly periodic shear layer vortex sheet (Eq. 10.10) with $\sigma=0.0375, \epsilon=0.05$, and (a) $t=0$, (b) $t>t_{c, \sigma}$. Even for large time $t$, the solution remains 2D, as shown by the projected side view $(\mathrm{c}) \ldots \ldots \ldots \ldots \ldots$

10.7 Extrapolated $(\sigma \rightarrow 0)$ singularity time for the 3D calculation of a $2 \mathrm{D}$ initial condition, as a function of spanwise position, $y_{s}$. Compare with corresponding $\epsilon=0.01$ in Fig. 10.5. . . . . . . . . .

10.8 3D normal mode initial condition and associated vortex lines. Flow is in the positive $x$ direction below the sheet, negative $x$ direction above the sheet. Vertical scale is exaggerated to demonstrate geometry: (a) view from above; (b) rotated view from above, revealing $2 \mathrm{D}$ geometry. Vortex lines are not exactly parallel to the $y$ axis. . . . . . . . . 111

11.1 Sketch of a planar vortex sheet with velocity jump $U \ldots \ldots 113$

C.1 Typical triangle in parameter space $(\Sigma) . \ldots \ldots \ldots \ldots \ldots$ 


\section{List of Tables}

6.1 Terms $T^{i, j}$ of the double summations $S_{i, j}$ needed to evaluate $C^{n}$. The first row represents the numerator and the first column represents the denominator of the summed term in each $S_{i, j}$, as exemplified in Eq. 6.10. 49

E.1 Summation values $S_{i, j}$ for $\sigma=1 / 10 \ldots \ldots \ldots \ldots \ldots \ldots \ldots$ 


\section{Chapter 1 Introduction}

Many fluid flows of interest are characterized by isolated regions of concentrated vorticity. Common examples include jets, vortex rings, free shear layers and turbulent flows. An underlying technique for computing such flows is to discretize the vorticity field with a Lagrangian mesh. These markers move with the fluid, and furthermore in an incompressible fluid, the velocity field can be calculated from the vorticity field and boundary conditions alone. Computation in this sense is efficient, as only a small portion of the flow domain is filled with the computational mesh. Diffusion of vorticity due to viscosity causes these isolated regions to grow with time, requiring the computational mesh also to grow and forcing the update of vorticity values in the mesh. This methodology is the basis of "vortex methods" (see reviews by Leonard [31] and Meiburg [34]), a broad category of computational fluid dynamics, the full span of which will not be reviewed here. Subsequent discussions in this thesis, though falling under the category of vortex methods, are limited to those involving the simulation of shear layers, or generally flows in which vorticity is localized to a three-dimensional surface.

At high Reynolds number and short times after creation, diffusion of vorticity from the previously mentioned isolated regions is negligible; in particular these flows are often modeled with zero viscosity - a common simplification which has been found to maintain the underlying dynamics in many respects [51]. In particular, free shear layers are often modeled as vortex sheets, surfaces of vorticity embedded in an otherwise irrotational, inviscid fluid. The free shear layer is a classic problem to study in fluid mechanics because the interaction of two different-speed fluids in contact with one another is one of the most basic dynamics of a fluid. Further motivation comes from the field of turbulence, in which high Reynolds numbers dominate and vortex sheet surfaces, with their associated roll-up, are seen as the mechanism for generating common vortex tube structures [58] (see [47] for a review of vortex methods 
in turbulence). This thesis is concerned with inviscid, constant-density fluids within which free shear layers, modeled as surfaces of vorticity, or vortex sheets, move as material elements. The fluid is considered to be irrotational save for the sheet surface itself. Henceforth the term 'vortex sheet' will refer to an isolated surface of vorticity, while the modifiers 'regularized' or 'smooth' will imply an approximation to the vortex sheet, as described below.

\subsection{Ill-Posedness of Vortex Sheets}

Numerical simulations of vortex sheet motion date back to Rosenhead's [49] calculations in 1931 in which he modeled, at fairly low precision, a sheet representing a perturbed planar shear layer as a row of two-dimensional (2D) point vortices. An attempt to refine Rosenhead's calculations by Birkhoff and Fisher [8] in 1959 was foiled by the discovery that increased resolution lead to irregular motion of the point vortices rather than convergence to a smooth solution.

Indeed, the linear stability of flat, 2D vortex sheets is ill-posed in the sense of Hadamard [17] - perturbations grow linearly with their wavenumber (this particular instability is known as the Kelvin-Helmholtz instability). Furthermore, the non-linear evolution of $2 \mathrm{D}$ vortex sheets was shown to develop a singularity (infinite surface curvature and a cusp in the sheet strength) in finite time by Moore [38], with further refinement by others $[13,28,35,55]$. Singularity formation has also been observed for axi-symmetric vortex sheets [40,41] and suggested for fully 3D vortex sheets [23, 24], though in each of these cases only specific initial conditions are considered and the results cannot be considered general. In addition, the results in the fully $3 \mathrm{D}$ case are only suggestive and it is not fully understood what effects the added spatial freedom has, if any, on singularity development. In general the implications of ill-posedness and singularity formation on the motion of vortex sheets past the singularity time is not rigorously understood; though it is entirely possible that after the singularity time a weak solution exists which may be calculated with a consistent regularization of the problem. Below we present a brief review of past regularization techniques and 
then focus on one which will be utilized in this thesis.

\subsection{Regularization of Vortex Sheet Motion}

Several methods have been introduced since 1959 which attempt to regularize the vortex sheet problem, potentially allowing calculations past the singularity time. A large collection of techniques are based on Rosenhead's [48] modification of the Biot-Savart kernel, in which a length scale parameter $\sigma$ is introduced, smoothing the kernel's behavior. This approximate Biot-Savart kernel effectively smoothes the vorticity distribution over a length scale $\sigma$. When applied to 2D point vortices, this smoothing technique produces "vortex blobs" $[3,12]$, the motion of which converges to a distinct sheet profile, both before and after the singularity time, as the number of vortex blobs increases; additionally, the solution converges for both finite values of the regularization parameter and apparently in the limit as the parameter tends to zero [27]. Extension of the vortex blobs to 3D line vortices, results in what is referred to as vortex "filaments" $[4,26,32]$. Large numbers of filaments are combined adjacent to one another to discretize a $3 \mathrm{D}$ vortex sheet. 3D regularized vortex sheets have also been represented by particles [60], a triangulated surface [2], and a level set method [20]. Of particular interest, regularized vortex sheet models utilizing finite sheet thickness [5, 37], Rosenhead's kernel smoothing [27] and viscosity [57] have been shown to converge to vortex sheet motion at times before the singularity formation, as the pertinent regularization parameter tends to zero. Incorporation of surface tension terms into the model [45] (1) eliminates roll-up completely with strong surface tension, but (2) introduces a new singularity as the interface collides with itself under weak surface tension [22]. The vortex-in-cell technique has been shown to generate roll-up past the singularity time with outer turns that converge to those of vortex blob calculations, as the regularization parameter tends to zero [56]. Explicit time dependence has been eliminated by examining self-similar vortex sheet rollup $[44,46,52]$, but matching this solution to Moore's sheet profile at the singularity time does not seem possible. 
We now focus on Rosenhead's modification of the Biot-Savart kernel as a regularization technique. In this shear layer model, a length scale $\sigma$ is introduced in the denominator of the Biot-Savart kernel, smoothing its behavior (for $\sigma>0$ ). The true (original) kernel is regained in the limit of $\sigma \rightarrow 0$. Regularization with $\sigma>0$ not only attenuates the unbounded growth rates at large wave number but also removes the finite time singularity, allowing computation of long-time evolution, provided sufficient accuracy [27]. The price is, of course, that the Euler equations with an embedded vorticity surface are no longer being simulated, but rather some effective model equation defined by the regularization. An important issue to address, then, is the relation between (Rosenhead's) regularized vortex sheets and (a) incompressible Euler calculations, (b) incompressible Navier-Stokes calculations, and (c) experimental observation.

\subsection{Suitability of the Smooth Vortex Sheet Model}

Krasny [29] performed several 2D calculations with the smooth Biot-Savart kernel to compare with experimental observations of the wake behind a cylinder in a $2 \mathrm{D}$ thin-film flow. The simulations, with moderate values of the smoothing parameter $(\sigma=0.3)$, are shown to capture important dynamical features of the experimental wake. Other $2 \mathrm{D}$ simulations (with $\sigma=0.025$ ) of the separated shear layer roll-up behind an impulsively driven flat plate produce a spiral in good agreement with the self-similar roll-up calculations of Pullin [44].

Nitsche and Krasny [42] performed axi-symmetric calculations with the smooth Biot-Savart kernel in order to model a previous experiment by Didden [15], in which a vortex ring was generated by the impulsive motion of a piston through a cylinder. Experimental visualization was provided by a thin, dyed column of fluid spanning the diameter of the cylinder, as seen in the top half of Fig. 1.1. The simulation (bottom half of Fig. 1.1) clearly shows good qualitative agreement with the experiment. Furthermore, the ring's path is correctly predicted, though its velocity is approximately $15 \%$ faster than in the experiment. The shape of the outer turns of the roll-up is 

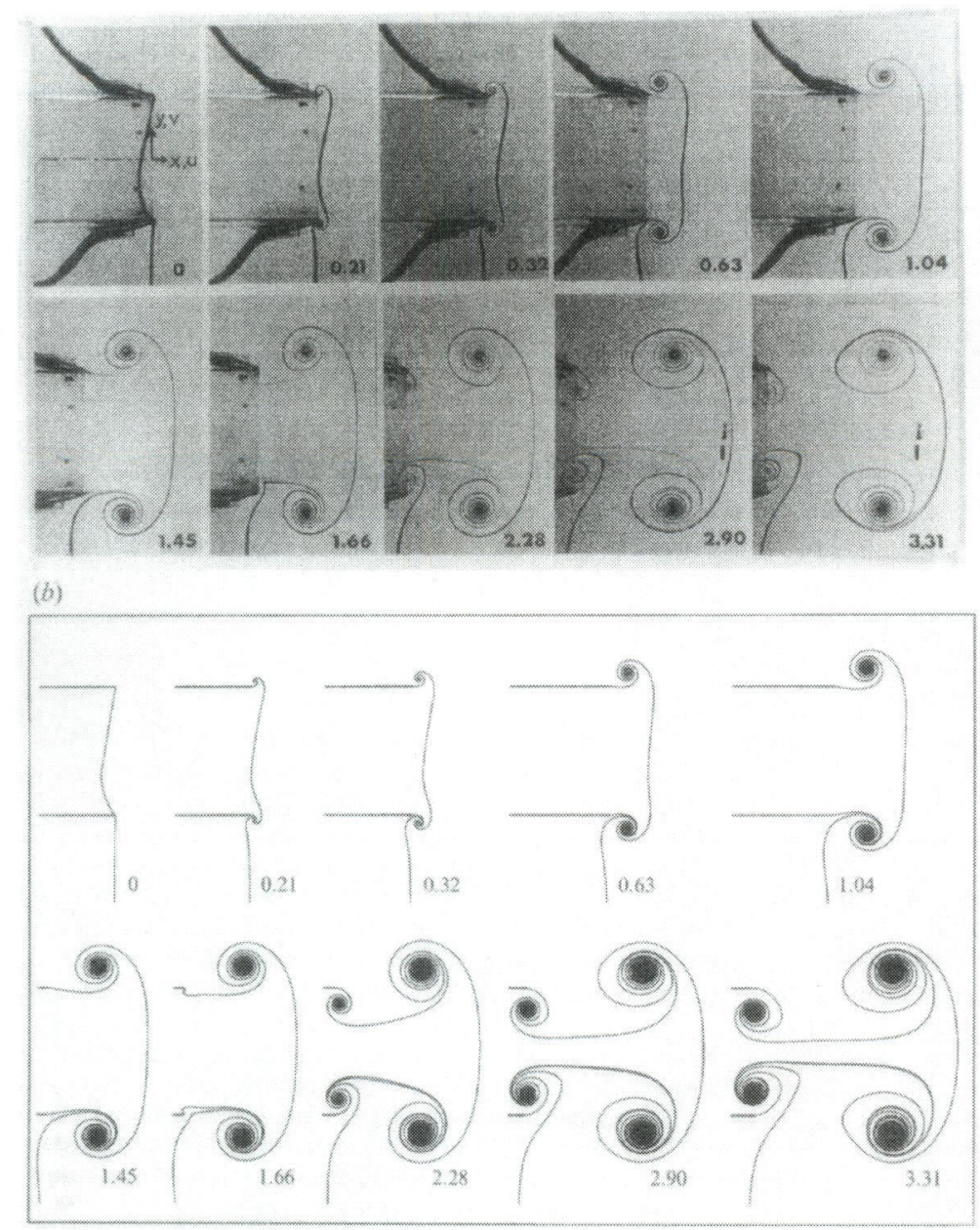

Figure 1.1: Comparison of vortex ring formation (reproduced from [42]). Experiment visualization above with dyed material line of fluid. Simulation below utilizing smooth kernel $(\sigma=0.2)$ depicting calculated material line.

fairly insensitive to the smoothing parameter $\sigma$, but the slight changes seen as $\sigma \rightarrow 0$ improve the agreement with experiment.

Tryggvason et al. [57] performed calculations of 2D periodic shear layer roll-up with two methods: (1) a viscous incompressible Navier-Stokes solver characterized by Reynolds number and initial shear layer thickness, and (2) the vortex blob method characterized by the Biot-Savart kernel smoothing parameter, $\sigma$. The viscous simulation was given an initial condition to precisely match that of the regularized vortex sheet with specified $\sigma$. Spanning a range of Reynolds numbers $\left(10^{3}\right.$ to $\left.2 \cdot 10^{4}\right)$ and thicknesses ( $\sigma=0.32$ to 0.08 ), their results imply that the limit of high Reynolds 
number and small initial thickness in the viscous simulations is the same as that for the vortex blob method as $\sigma$ tends to zero. Also, at moderate Reynolds number and initial thickness, the outer turns of the roll-up region agree quite well between the two methods. However, in this moderate thickness case, the methods do not agree as well in the vortex core. Incompressible Euler simulations (2D) with initial conditions matched to the regularized vortex sheet reveal a similar correspondence: agreement in the outer turns, but differences in the roll-up core.

Overall, Rosenhead's smoothing regularization proves to be a highly functional method for approximating the motion of vortex sheets, both before and after the singularity time. Furthermore, evidence suggests the regularized equations provide a good model of real flow, deserving of new refinements and applications.

\subsection{D Regularized Vortex Sheets}

$3 \mathrm{D}$ simulations to date have generally involved sufficient smoothing of the BiotSavart kernel to damp the smallest wavelengths, completely avoiding singularity formation issues. Each method is forced, however, to devote more effort than its 2D counterpart with regard to "mesh" refinement, in order to maintain accurate vorticity field representation despite 3D Lagrangian deformation. Of course, the beauty of a $3 \mathrm{D}$ simulation is the ability to capture vortex stretching and fully $3 \mathrm{D}$ instabilities not found in any $2 \mathrm{D}$ calculations. The computational portion of the present work is an extension of [2], adding higher order sheet representation; global, automatic mesh refinement; faster multi-pole velocity evaluation; and singly and doubly periodic surface geometries.

We also present a new numerical technique for estimating the critical time (of singularity formation) for vortex sheets with arbitrary initial configuration, which is shown to work with the smooth Biot-Savart kernel. Finally, following a lead from calculations, we present an extension of Moore's [38] analysis, showing that his 2D singularity prediction also applies to $3 \mathrm{D}$ normal mode perturbations of the planar shear layer. 


\subsection{Overview}

The following chapter provides the necessary background material and describes the governing equations for vortex sheets as well as invariants of the system. Chapter 3 is an overview of the computational technique, which leads into more detail of mesh generation, fast velocity evaluation and periodic velocity evaluation in the subsequent chapters. Chapter 7 is an analysis of the remaining computational issues. Chapter 8 presents several case studies as examples of what can be simulated with this regularized vortex sheet implementation. Chapter 9 is an application of the regularized vortex sheet model to the planar shear layer with 3D perturbations. Chapters 10 and 11 cover a specific study into certain aspects of singularity development in vortex sheets. Chapter 12 concludes this thesis with a discussion and presents possible directions for future work. 


\section{Chapter 2 Theoretical Development}

A brief derivation of the equations governing the evolution of vortex sheets is given in the following three sections. The first two provide the necessary background information from differential surface geometry and fluid kinematics respectively.

\subsection{Parametric Surface}

We employ a parametric surface description

$$
\mathbf{X}(\xi, \eta)=\left(\begin{array}{c}
X \\
Y \\
Z
\end{array}\right)(\xi, \eta)
$$

where, as shown in Fig. 2.1, $(X, Y, Z)$ represents a point in $(x, y, z)$ space on the surface and $(\xi, \eta) \in \Sigma$ is a point in a 2-D isoparametric space. The first fundamental coefficients [59] of the mapping are

$$
\begin{aligned}
E & =\mathbf{X}_{\xi} \cdot \mathbf{X}_{\xi} \\
F & =\mathbf{X}_{\xi} \cdot \mathbf{X}_{\eta} \\
G & =\mathbf{X}_{\eta} \cdot \mathbf{X}_{\eta}
\end{aligned}
$$

so that an infinitesimal segment in parameter space with components $d \boldsymbol{\xi}=(d \xi, d \eta)$ is mapped to a corresponding segment of length $d X$ in physical space, where

$$
d X^{2}=E d \xi^{2}+2 F d \xi d \eta+G d \eta^{2}
$$




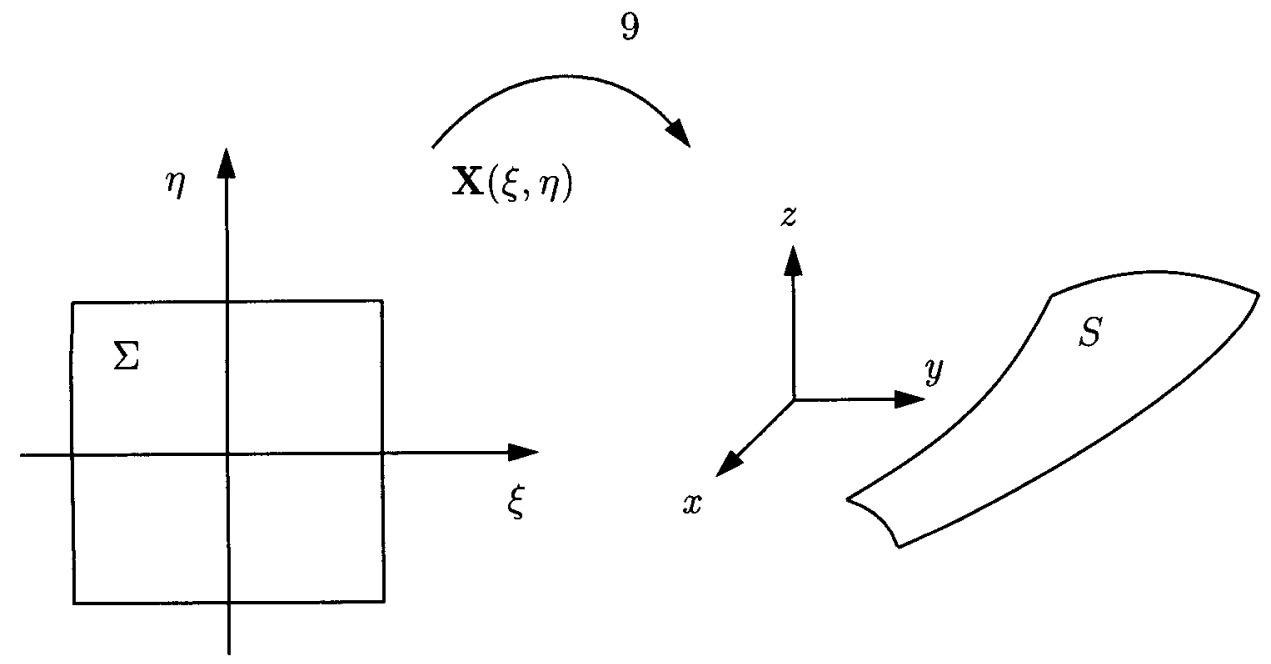

Figure 2.1: Mapping from parameter to physical space.

Subscripts $\xi$ and $\eta$ denote partial differentiation with respect to the corresponding parameter. Two other useful quantities are the Jacobian of the mapping,

$$
J=\left|\mathbf{X}_{\xi} \times \mathbf{X}_{\eta}\right|=\sqrt{E G-F^{2}}
$$

and unit surface normal

$$
\mathbf{n}=\mathbf{X}_{\xi} \times \mathbf{X}_{\eta} / J
$$

Points for which $J=0$ are singular, such as the north and south poles of a sphere parameterized with latitude and longitude. The Gaussian curvature $K$ is

$$
K=\kappa_{a} \kappa_{b}=\frac{L N-M^{2}}{J^{2}}
$$

where $\kappa_{a}$ and $\kappa_{b}$ are the principal curvatures of the surface [59], and $L, M$, and $N$ are the second fundamental coefficients defined by

$$
\begin{aligned}
L & =\mathbf{n} \cdot \mathbf{X}_{\xi \xi} \\
M & =\mathbf{n} \cdot \mathbf{X}_{\xi \eta} \\
N & =\mathbf{n} \cdot \mathbf{X}_{\eta \eta} .
\end{aligned}
$$


The mean curvature $\mu$ is

$$
\mu=\frac{1}{2}\left(\kappa_{a}+\kappa_{b}\right)=\frac{E N+G L-2 F M}{2\left(E G-F^{2}\right)},
$$

and the square of the total curvature $\kappa_{T}$ is given by

$$
\kappa_{T}^{2}=\kappa_{a}^{2}+\kappa_{b}^{2}=2\left(2 \mu^{2}-K\right)
$$

Note that in other literature the total curvature is sometimes defined as the integral of the Gaussian curvature over the surface [59].

\subsection{The Velocity Field}

Consider an unbounded uniform density and inviscid fluid. Vorticity is defined as the curl of the velocity field

$$
\boldsymbol{\omega}=\nabla \times \mathbf{u}
$$

and circulation around a closed loop $\partial \mathcal{D}$ (vorticity flux through $\mathcal{D}$ ) is

$$
\Gamma=\oint_{\partial \mathcal{D}} \mathbf{u} \cdot d \boldsymbol{\ell}=\int_{\mathcal{D}} \boldsymbol{\omega} \cdot \mathbf{n} d \mathcal{D}
$$

Combining the curl of Eq. 2.10 with the incompressibility condition $(\nabla \cdot \mathbf{u}=0)$ gives a Poisson equation for velocity,

$$
\nabla^{2} \mathbf{u}=-\nabla \times \boldsymbol{\omega}
$$

the solution of which is

$$
\mathbf{u}(\boldsymbol{x})=\frac{1}{4 \pi} \int_{V} \frac{\boldsymbol{\omega}\left(\boldsymbol{x}^{\prime}\right) \times\left(\boldsymbol{x}-\boldsymbol{x}^{\prime}\right)}{\left|\boldsymbol{x}-\boldsymbol{x}^{\prime}\right|^{3}} d^{3} \boldsymbol{x}^{\prime}
$$




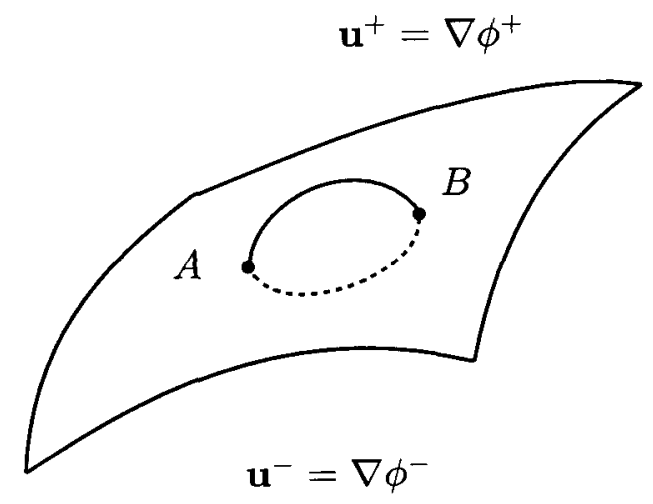

Figure 2.2: Vortex sheet dividing an ideal fluid.

where $V$ is the vorticity containing region [51]. A homogeneous solution of Eq. 2.12 also exists, in the form of a Laplacian velocity potential; this solution is omitted as it is not relevant to the remaining discussion.

\subsection{Vortex Sheet Motion}

As discussed in Chapter 1, we wish to restrict the vorticity field to thin layers in the fluid, which become vortex sheets in the limit as the thickness tends to zero. Of course in this regime viscous effects are lost, but the vortex sheet provides a good model for the large scale dynamics of a flow [42]. In order for the strength of the sheet (that is, the magnitude of the difference in flow velocity on either side) to remain constant throughout this limiting process, the magnitude of the vorticity in the layer must be increased while the layer thickness is decreased such that their product remains constant [51]. Thus the vorticity is in fact singular on the sheet surface. For parameterized vortex sheets, the vorticity vector takes a particular form, transforming Eq. 2.13 into an equation for the evolution of the sheet itself $[2,10,25]$. Consider a vortex sheet which divides an otherwise irrotational, inviscid fluid into two regions, as shown in Fig. 2.2. The solenoidal velocity in each region is given completely by velocity potentials, i.e., $\mathbf{u}^{+}=\nabla \phi^{+}$and $\mathbf{u}^{-}=\nabla \phi^{-}$. Vorticity $\boldsymbol{\omega}$ must 
lie tangent to the surface and may be defined as

$$
\boldsymbol{\omega}=\boldsymbol{\gamma} \delta(n)
$$

where $n$ is the distance normal to the sheet and $\delta$ is the Dirac delta function. $\gamma$ is the strength of the sheet given by

$$
\gamma=\mathbf{n} \times[\mathbf{u}]
$$

where $\mathbf{n}$ is the unit surface normal (pointing to the + side) and the brackets denote the jump across the sheet, i.e., $[\mathbf{u}]=\mathbf{u}^{+}-\mathbf{u}^{-}$, evaluated on the sheet surface. Consider the circulation around a closed curve $\mathcal{C}$ piercing the vortex sheet through points $A$ and $B$ in a clockwise sense. As the velocities on either side of the sheet have potentials and vorticity is non-zero only on the sheet, circulation depends only on the location of points $A$ and $B$, i.e.,

$$
\begin{aligned}
\Gamma_{\mathcal{C}} & =\int_{\mathcal{C}} \mathbf{u} \cdot d \boldsymbol{\ell} \\
& =\int_{A}^{B} \mathbf{u}^{+} \cdot d \boldsymbol{\ell}+\int_{B}^{A} \mathbf{u}^{-} \cdot d \boldsymbol{\ell} \\
& =\int_{A}^{B}[\mathbf{u}] \cdot d \boldsymbol{\ell} \\
& =\int_{A}^{B}\left(\nabla \phi^{+}-\nabla \phi^{-}\right) \cdot d \boldsymbol{\ell} \\
& =[\phi](B)-[\phi](A) .
\end{aligned}
$$

If the vortex sheet surface is represented by $\mathbf{X}(\xi, \eta)$, with $(\xi, \eta) \in \Sigma$, circulation can be regarded as a point-wise quantity by taking $A$ to be a fixed reference location in parameter space, i.e., $\Gamma_{0}=[\phi](A)$ and

$$
\Gamma(\xi, \eta)=[\phi](\xi, \eta)-\Gamma_{0}
$$


With this definition, $\Gamma$ is a single valued function of $(\xi, \eta)$. Thus, some care must be taken when considering sheets that contain irreducible curves such as a torus and infinite cylinder [51]. Fortunately, only the circulation gradients are required in the following discussion.

Using Eq. 2.5 as the normal direction, the vector identity $(a \times b) \times c=(a \cdot c) b-(b \cdot c) a$, and noting $\Gamma_{\xi}=[\nabla \phi] \cdot \mathbf{X}_{\xi}$ and $\Gamma_{\eta}=[\nabla \phi] \cdot \mathbf{X}_{\eta}$, it follows from Eq. 2.15 that

$$
\gamma(\xi, \eta)=\frac{\Gamma_{\xi} \mathbf{X}_{\eta}-\Gamma_{\eta} \mathbf{X}_{\xi}}{\left|\mathbf{X}_{\xi} \times \mathbf{X}_{\eta}\right|}
$$

Thus, Eq. 2.13 becomes

$$
\mathbf{u}(\boldsymbol{x})=\frac{1}{4 \pi} \int_{\Sigma} \frac{\mathbf{W}^{\prime} \times\left(\boldsymbol{x}-\mathbf{X}^{\prime}\right)}{\left|\boldsymbol{x}-\mathbf{X}^{\prime}\right|^{3}} d \Sigma^{\prime}
$$

where

$$
\mathbf{W}=\Gamma_{\xi} \mathbf{X}_{\eta}-\Gamma_{\eta} \mathbf{X}_{\xi}
$$

and all upper case vectors are functions of $(\xi, \eta, t)$, where $t$ is time. An evolution equation for the sheet is defined by restricting $\boldsymbol{x}$ to lie on $\mathbf{X}(\xi, \eta)$. From Eq. 2.19 we have

$$
\dot{\mathbf{X}}=\mathbf{u}(\mathbf{X})
$$

Note that in this case the integral must be interpreted as giving the average of velocities allowed when $x$ approaches the sheet from either side; that is Eq. 2.19 must be interpreted as as a Cauchy Principal Value or Hadamard finite part integral. For a related discussion and a specific calculation involving a steady $3 \mathrm{D}$ vortex sheet, see Moore and Pullin [39]. Although the sheet vorticity $\mathbf{W}$ changes with time, it is well known that $\Gamma$ is invariant, since vortex lines (those everywhere tangent to $\boldsymbol{\omega}$ ) move with the sheet [51]. Thus, in this formulation, no explicit updating of vorticity is required. Examining Eq. 2.20 it is clear that with a specific parameterization 
the circulation can be a function of only one variable $\left(\Gamma_{\eta}=0\right)$, leaving the parameterized vorticity as simply $\mathbf{W}=\Gamma_{\xi} \mathbf{X}_{\eta}$ (this approach is utilized in Appendix B). This simplifies the integrand of Eq. 2.19 slightly, but in turn complicates the manual parameterization of general surfaces.

Experience in 2-D [28] shows that time integration of Eq. 2.21 is inherently unstable due to amplification of round-off error, and a means of regularization is necessary. As discussed in Chapter 1, one method to remedy the ill-posedness is to introduce a length scale $\sigma$ into the denominator of the Biot-Savart kernel, smoothing its behavior. The modified equation is

$$
\mathbf{u}_{\sigma}(\boldsymbol{x})=\frac{1}{4 \pi} \int_{\Sigma} \frac{\mathbf{W}^{\prime} \times\left(\boldsymbol{x}-\mathbf{X}^{\prime}\right)}{\left[\left|\boldsymbol{x}-\mathbf{X}^{\prime}\right|^{2}+\sigma^{2}\right]^{3 / 2}} d \Sigma^{\prime}
$$

which as before leads to an evolution equation for the surface:

$$
\dot{\mathbf{X}}=\mathbf{u}_{\sigma}(\mathbf{X})
$$

This is precisely Rosenhead's kernel smoothing as discussed in Chapter 1. Clearly Eq. 2.19 is regained in the limit as $\sigma \rightarrow 0$ in Eq. 2.22. Recall, the term 'vortex sheet' is used in this thesis to refer to the $\sigma=0$ limit while the modifiers 'regularized' or 'smooth' are applied to distinguish the approximated surface and motion described by Eq. 2.23 .

With the modified, smooth kernel of Eq. 2.22, the velocity field is continuous across the regularized sheet surface and the integral involved is no longer complicated by different limiting values when approached from either side. Recall other vortex sheet simulations with the same regularization have proven to be good models of various flow phenomena (e.g., [42]) and the limiting case as $\sigma \rightarrow 0$ approaches that of (1) true vortex sheets before the singularity time [27] and also (2) thin viscous layers as the Reynolds number is increased and initial thickness decreased [57]. The relation of $\sigma$ to other aspects of the current smooth vortex sheet implementation is discussed in $\S 2.5, \S 5.1, \S 6.1, \S 7.4, \S 7.5, \S 8.1$, and $\S 10$. 
All variables mentioned hereafter are assumed to be non-dimensional, scaled with appropriate combinations of a characteristic length $L_{0}$ and circulation $\Gamma_{0}$, unless noted otherwise. An initial value problem is now clearly defined: calculate the time evolution of $\mathbf{X}(\xi, \eta, t)$, subject to Eq. 2.23 , given the initial sheet position $\mathbf{X}(\xi, \eta, 0)$ and relative circulation $\Gamma(\xi, \eta)$.

\subsection{Surface Types: SWP0, SWP1 and SWP2}

In order to be computationally tractable, only surfaces that can be described with a compact parameter space domain $\Sigma$ are considered. Specifically, we take $(\xi, \eta) \in[-\pi . . \pi]$ for all cases. Physical space surfaces with infinite extent are thus sliced at regular intervals and constrained to periodic motion. Categorization of surfaces is based on the prescribed constraints in parameter space. The first surface type considered is that which results from a mapping function $\mathbf{X}$, constrained to be simply periodic in both $\xi$ and $\eta$. $\mathbf{X}$ is then a torus-like surface, though the mapping is general enough to describe an arbitrarily distorted torus. This type of surface is used, for example, to follow the motion of hollow-core vortex rings and will be referred to henceforth as SWP0, an acronym for "surface with periodicity 0." Eq. 2.22 can be used directly to calculate the evolution of the toroidal surface, but for the following two surface types the nominally infinite domains must be accounted for explicitly.

The second surface type (SWP1) is similar to the first, but forces the first component of $\mathbf{X}$ to have an additional linear variation in $\xi$. This results in a singly periodic train of surfaces in physical space, connected smoothly from end to end. This type of surface is used to track hollow-core vortex tubes, jets and combinations of the two. As an example, consider the parameter space $(\xi, \eta) \in(-\infty . . \infty,-\pi . . \pi)$, where the periodic-train surface is sliced at intervals of $2 \pi$ in $\xi$, unit length in $x$, and points 
along the $x$ axis. Then Eq. 2.22 becomes

$$
\begin{aligned}
\mathbf{u}_{\sigma}(\boldsymbol{x}) & =\frac{1}{4 \pi} \int_{-\pi}^{\pi} \int_{-\infty}^{\infty} \frac{\mathbf{W}^{\prime} \times\left(\boldsymbol{x}-\mathbf{X}^{\prime}\right)}{\left[\left|\boldsymbol{x}-\mathbf{X}^{\prime}\right|^{2}+\sigma^{2}\right]^{3 / 2}} d \xi^{\prime} d \eta^{\prime} \\
& =\frac{1}{4 \pi} \int_{-\pi}^{\pi} \int_{-\pi}^{\pi} \sum_{m=-\infty}^{\infty} \frac{\mathbf{W}^{\prime} \times\left[\boldsymbol{x}-\left(\mathbf{X}^{\prime}+m \hat{\mathbf{i}}\right)\right]}{\left[\left|\boldsymbol{x}-\left(\mathbf{X}^{\prime}+m \hat{\mathbf{i}}\right)\right|^{2}+\sigma^{2}\right]^{3 / 2}} d \xi^{\prime} d \eta^{\prime}
\end{aligned}
$$

where $\hat{\mathbf{i}}$ is the unit vector along the $x$ axis. Direct integration of Eq. 2.24 now requires evaluation of the following summations:

$$
s_{1}(b, c)=\sum_{m=-\infty}^{\infty} \frac{1}{\left[(m-b)^{2}+c^{2}\right]^{3 / 2}}, \quad s_{2}(b, c)=\sum_{m=-\infty}^{\infty} \frac{m}{\left[(m-b)^{2}+c^{2}\right]^{3 / 2}},
$$

where $b$ and $c$ are combinations of variables from Eq. 2.24, treated as constant in the summations. As described in Chapter 6, there is an alternative, superior method to capture the influence of periodic images.

The last surface type (SWP2) forces the first and second components of $\mathbf{X}$ to have additional linear variations in $\xi$ and $\eta$, respectively. This results in a doubly periodic surface which splits physical space into two halves, clearly useful for examining standard shear layer configurations. To show the parameterization clearly, consider the parameter space $(\xi, \eta) \in(-\infty . . \infty,-\infty . . \infty)$, where the doubly periodic surface is sliced at intervals of $2 \pi$ in $(\xi, \eta)$ and unit length in $(x, y)$. Then Eq. 2.22 becomes

$$
\begin{aligned}
\mathbf{u}_{\sigma}(\boldsymbol{x}) & =\frac{1}{4 \pi} \int_{-\pi}^{\pi} \int_{-\infty}^{\infty} \frac{\mathbf{W}^{\prime} \times\left(\boldsymbol{x}-\mathbf{X}^{\prime}\right)}{\left[\left|\boldsymbol{x}-\mathbf{X}^{\prime}\right|^{2}+\sigma^{2}\right]^{3 / 2}} d \xi^{\prime} d \eta^{\prime} \\
& =\frac{1}{4 \pi} \int_{-\pi}^{\pi} \int_{-\pi}^{\pi} \sum_{m=-\infty}^{\infty} \sum_{n=-\infty}^{\infty} \frac{\mathbf{W}^{\prime} \times\left[\boldsymbol{x}-\left(\mathbf{X}^{\prime}+m \hat{\mathbf{i}}+n \hat{\mathbf{j}}\right)\right]}{\left[\left|\boldsymbol{x}-\left(\mathbf{X}^{\prime}+m \hat{\mathbf{i}}+n \hat{\mathbf{j}}\right)\right|^{2}+\sigma^{2}\right]^{3 / 2}} d \xi^{\prime} d \eta^{\prime}
\end{aligned}
$$

where $\mathbf{i}$ and $\mathbf{j}$ are unit vectors in the $x$ and $y$ directions, respectively. Direct integration of Eq. 2.26 requires more summations than for the SWP1 surface, and thus are not mentioned here in light of the forthcoming Chapter 6. 
Note that specific surface geometries are omitted due to their non-trivial parameterizations. For example, a spherical surface is omitted because of the coordinate singularities in its parameterization associated with the poles, as noted in $\S 2.1$. This surface type would be useful, however, for examining the motion of shocked density bubbles [21].

\subsection{Invariants}

Uniform density, inviscid fluids with an embedded vortex sheet maintain an infinite number of invariant quantities [51], though a select group of integrated quantities provide a good set of diagnostics for a numerical method. It is important to recognize here the difference between invariants of vortex sheet flow and those of a regularized vortex sheet flow [60]. Due to the specific regularization taken in Eq. 2.22, it is not obvious that regularized vortex sheet flows maintain any invariant quantities, even with the assumption of perfect spatial and temporal integrators. However, as highlighted in Appendix B, the following quantities, carefully regularized in the sense of Eq. 2.22, are preserved. Each invariant has an obvious counterpart for true vortex sheet flows. Unit density is assumed for the fluid. We introduce the subscript $\delta$ to refer explicitly to the velocity field $\left(\mathbf{u}_{\delta}\right)$ induced by a true vortex sheet with vorticity $\boldsymbol{\omega}_{\delta}=\boldsymbol{\gamma} \delta(n)$, as in Eq. 2.14 and Eq. 2.19 . 


$$
\text { Volume: } \quad V=\frac{1}{3} \int_{V_{S}} \nabla \cdot x d V=\frac{1}{3} \int_{\Sigma} \mathbf{X} \cdot\left(\mathbf{X}_{\xi} \times \mathbf{X}_{\eta}\right) d \Sigma
$$

Kinetic energy: $\quad T \equiv \frac{1}{2} \int_{V} \mathbf{u}_{\sigma} \cdot \mathbf{u}_{\delta} d V=\frac{1}{8 \pi} \iint_{\Sigma} \frac{\mathbf{W} \cdot \mathbf{W}^{\prime}}{\left|\mathbf{X}-\mathbf{X}^{\prime}\right|} d \Sigma d \Sigma^{\prime}$

$$
\text { Helicity: } \quad H \equiv \int_{V} \mathbf{u}_{\sigma} \cdot \boldsymbol{\omega}_{\delta} d V=\int_{\Sigma} \mathbf{U} \cdot \mathbf{W} d \Sigma
$$

Total vorticity: $\mathcal{W} \equiv \int_{V} \boldsymbol{\omega}_{\delta} d V=\int_{\Sigma} \mathbf{W} d \Sigma=\mathbf{0}$

Hydrodynamic impulse: $\quad \mathbf{I} \equiv \frac{1}{2} \int_{V} \boldsymbol{x} \times \boldsymbol{\omega}_{\delta} d V=\frac{1}{2} \int_{\Sigma} \mathbf{X} \times \mathbf{W} d \Sigma$

Angular impulse:

$$
\begin{aligned}
\mathbf{A} & \equiv \frac{1}{3} \int_{V} \boldsymbol{x} \times\left(\boldsymbol{x} \times \boldsymbol{\omega}_{\delta}\right) d V \\
& =\frac{1}{3} \int_{\Sigma} \mathbf{X} \times(\mathbf{X} \times \mathbf{W}) d \Sigma
\end{aligned}
$$

Here $V_{S}$ represents the volume enclosed by the vortex sheet surface, $V$ is all space with non-zero vorticity, and $\Sigma$ is the parameterized sheet surface. For SWP1 and SWP2 surface types, the above definitions need slight modification to account for physical space periodicity. 


\section{Chapter 3 Computational Development}

A summary of the computational technique used is given in the next paragraph; more details follow in the subsequent sections.

The integrand of Eq. 2.22 is approximated over an irregular, triangulated mesh covering the parameter space domain $\Sigma$, an example of which is depicted in the left of Fig. 3.1(a). Mesh refinement (resulting in non-uniform distribution of grid points) is essential in order to capture local areas of high deformation in the physical sheet which develop during the sheet evolution (see Fig. 3.2). Parameter space triangulation (both vertex placement and connection) is accomplished by an advancing front method. The vortex sheet surface is re-triangulated as needed, based on surface curvature and stretching, with triangle size and orientation conforming to local properties of the surface. Mesh post-processing techniques are implemented to increase desired characteristics of the mesh. The nine functions $\mathbf{X}(\xi, \eta), \mathbf{X}_{\xi}(\xi, \eta)$ and $\mathbf{X}_{\eta}(\xi, \eta)$ are interpolated in parameter space by a combination of cubic Bézier triangular patches [18], providing $C^{1}$ continuity between triangles in $\mathrm{X}$ and $C^{0}$ con-

tinuity in $\mathbf{X}_{\xi}, \mathbf{X}_{\eta}$. The spatial integration in Eq. 2.22 is approximated with Gauss quadrature points [14] positioned inside each triangle and time integration proceeds with standard Runge-Kutta methods. Note that in forthcoming figures, vortex sheet surfaces are, with one exception, depicted simply with their triangular mesh skeleton. In this piecewise planar representation, only the triangle vertices are necessarily on the interpolated sheet surface. An example of the smooth sheet surface is shown in Fig. 8.1(f).

\subsection{Surface Interpolation}

In order to evaluate the integrand of Eq. 2.22, $\mathbf{X}$ and its derivatives $\left(\mathbf{X}_{\xi}\right.$ and $\left.\mathbf{X}_{\eta}\right)$ must be interpolated within $\Sigma$. This could be done trivially with linear interpolants 
defined locally inside each triangle. Using local barycentric coordinates $\zeta_{1}, \zeta_{2}, \zeta_{3}$ to define a linear interpolant of the function $X$ in a particular triangle, with $X$ values $\left(X_{1}, X_{2}, X_{3}\right)$ at its vertices, gives

$$
X=\zeta_{1} X_{1}+\zeta_{2} X_{2}+\zeta_{3} X_{3}, \quad \zeta_{1}+\zeta_{2}+\zeta_{3}=1
$$

But this simple interpolant gives only $C^{0}$ continuity between triangles.

A higher order interpolant is given by a combination of cubic Bézier triangular patches defined over each triangle [18]. Again the patch is a function of local barycentric coordinates $\zeta_{1}, \zeta_{2}, \zeta_{3}$ and has the form

$$
\begin{aligned}
X\left(\zeta_{1}, \zeta_{2}, \zeta_{3}\right)= & \zeta_{1}^{3} b_{3,0,0}+\zeta_{2}^{3} b_{0,3,0}+\zeta_{3}^{3} b_{0,0,3} \\
& +3 \zeta_{1}^{2} \zeta_{2} b_{2,1,0}+3 \zeta_{1}^{2} \zeta_{3} b_{2,0,1}+3 \zeta_{1} \zeta_{2}^{2} b_{1,2,0} \\
& +3 \zeta_{1} \zeta_{3}^{2} b_{1,0,2}+3 \zeta_{2}^{2} \zeta_{3} b_{0,2,1}+3 \zeta_{2} \zeta_{3}^{2} b_{0,1,2} \\
& +6 \zeta_{1} \zeta_{2} \zeta_{3} \frac{\zeta_{2}^{2} \zeta_{3}^{2} b_{1,1,1}^{1}+\zeta_{1}^{2} \zeta_{3}^{2} b_{1,1,1}^{2}+\zeta_{1}^{2} \zeta_{2}^{2} b_{1,1,1}^{3}}{\zeta_{2}^{2} \zeta_{3}^{2}+\zeta_{1}^{2} \zeta_{3}^{2}+\zeta_{1}^{2} \zeta_{2}^{2}}
\end{aligned}
$$

where the constants $b_{i, j, k}$ and $b_{1,1,1}^{i}$ depend on the values of the function to be interpolated (for example $X$ ) and the first derivatives $\left(X_{\xi}\right.$ and $\left.X_{\eta}\right)$, at the triangle vertices.

Unfortunately, the first derivatives of the function to be interpolated must be specified at the triangle vertices. Thus, before the constants $b_{i, j, k}$ and $b_{1,1,1}^{i}$ are calculated, derivatives are estimated at each vertex (in $\Sigma$ ) by means of a parabolic least square fit of function values, taken from the vertices of the local triangle and neighboring triangles. Care is taken to ensure enough data for the fit by including vertices from the next ring of adjacent triangles if necessary. Once the fit is constructed, nodal derivative estimates are extracted from the analytic form of the paraboloid. Once function values and derivative estimates are known for the three vertices of a triangle, the constants $b_{i, j, k}$ and $b_{1,1,1}^{i}$ are trivially calculated (see [18]), and the analytic interpolant for the function is defined as above. The function's derivatives with respect to $\xi$ and $\eta$ are obtained from the analytical derivative of the interpolant (see 
Appendix $\mathrm{C})$. The functional interpolant is found to have $O\left(\Delta X^{3}\right)$ accuracy while its derivatives are only $O\left(\Delta X^{2}\right)$ accurate (see $\left.\S 7.4\right)$.

\subsection{Integration}

In addition to the interpolant, the time stepping method and number of Gauss points per triangle control the order of accuracy of each computation. Time stepping is done with one of the Runge-Kutta methods, selectable for second to fourth order accuracy. The time step size is regulated by three concerns: (1) stability, (2) preservation of invariants, and (3) control over triangle edge rotation and straining. The third is implemented by defining the strain for each edge as

$$
\epsilon=\frac{|\Delta U| \Delta t}{L}
$$

where $L$ is the original length of the edge (in physical space) and $\Delta U$ is the difference in velocities of the edge endpoints. The time step is varied so as to maintain $\epsilon<\epsilon_{0}$ for every edge. The parameter $\epsilon_{0}$ is chosen before computation so that invariants are maintained to the desired accuracy (as far as time integration contributes).

The integral in Eq. 2.22 is approximated for each triangle vertex in $\Sigma$. The integrand is evaluated at the Gauss quadrature points for each triangle, finally determining the global influence of the surface on each vertex. Thus, with this straightforward implementation, calculation of the surface velocity field is an $O\left(N^{2}\right)$ process. The spatial integration order is easily varied by changing the number of Gauss points used in each triangle ( $2^{\text {nd }}$ to $5^{\text {th }}$ order in $\Delta X$ with 1 to 6 points). The $O\left(N^{2}\right)$ discretization of Eq. 2.23 is

$$
\frac{\partial \mathbf{X}}{\partial t}\left(\xi_{i}, \eta_{i}\right)=\frac{1}{4 \pi} \sum_{n=1}^{N} \sum_{\left(\xi_{m}, \eta_{m}\right) \in G_{n}} w_{m} \frac{\mathbf{W}_{m} \times\left(\mathbf{X}_{i}-\mathbf{X}_{m}\right)}{\left[\left|\mathbf{X}_{i}-\mathbf{X}_{m}\right|^{2}+\sigma^{2}\right]^{3 / 2}}
$$

where $n$ is the index running over the triangles of the sheet surface, $\mathbf{X}_{m}$ implies $\mathbf{X}\left(\xi_{m}, \eta_{m}\right)$ and likewise with $\mathbf{W}_{m}$ and $\mathbf{X}_{i}$. The term $w_{m}$ represents the weight assigned 
to the $m^{\text {th }}$ Gauss point $\left(\xi_{m}, \eta_{m}\right)$ for triangle $n$, and $G_{n}$ refers to the set of Gauss points for the $n^{\text {th }}$ triangle. The double summation is performed for each vertex $i$ of the sheet surface. Smaller values of $\sigma$, or a tightly folded vortex sheet surface, lead to near singular behavior of the integrand, forcing more Gauss points or more triangles in order to maintain accuracy in spatial integration.

\subsection{Parallelism}

Both the $O\left(N^{2}\right)$ integration process described in the previous section, and the faster methods described in Chapter 5 are well-suited for shared memory, multiple thread parallelism. Most commercial modern-day compilers on computers with multiple CPUs and the ability to access a shared memory block, provide commands for flagging specific loops to be broken into $P$ divisions, where $P$ is the number of CPUs. As an example, consider the typical $O\left(N^{2}\right)$ velocity evaluation loop:

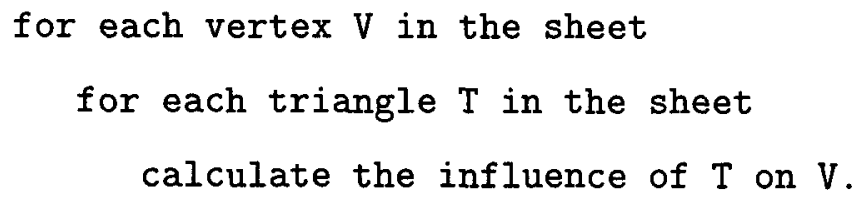

In this case well-suited means that each iteration through the outer (V) loop is independent of all other iterations, which allows us to write:

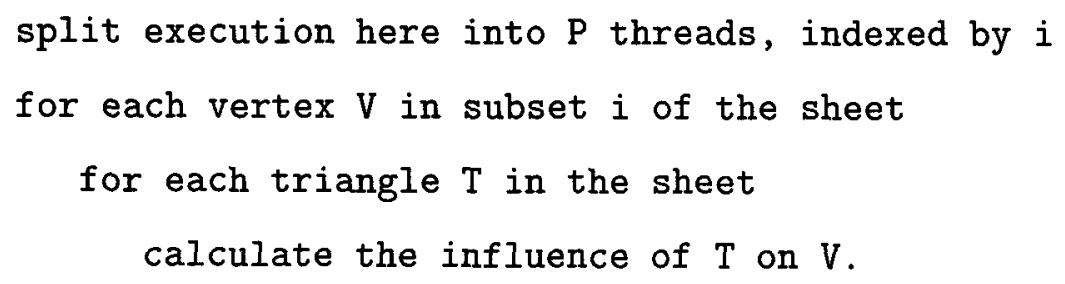

This velocity evaluation loop is of course just a portion of the entire program, but it is the most CPU intensive. Hence, only this section is parallelized. The effectiveness of this technique is discussed in $\S 7.7$. 


\subsection{SWP1 Summation Approximation}

As noted in $\S 2.4$, evaluation of the evolution equation for SWP1 surfaces requires the ability to compute the summations in Eq. 2.25. Fortunately, the two summations can be calculated once, before any runs, and stored at machine precision in a two-dimensional look-up table with the parameters $(b, c)$ as the axes. This is feasible because only a compact space from the $(b, c)$ domain is required in order to perform the numerical integration. Data is interpolated from the table with a $3^{\text {rd }}$ order accurate interpolant [1]. Keep in mind that these summations and the evolution equation modified for SWP1 surfaces (Eq. 2.24) are only relevant to the $O\left(N^{2}\right)$ spatial integration technique. An alternate method, though not implemented in this thesis, is described in Chapter 6.

\subsection{Trigger for Mesh Refinement}

As revealed in Fig. 3.2, there is an absolute need for mesh refinement to reach long times in a typical evolution. At which point in time (i.e., which time step) the remeshing occurs is non-trivial to optimize, however. The remesh process is significantly less time consuming than the velocity evaluation, so frequent remeshing is not a burden in terms of CPU time. However, each remesh does require interpolation for the new surface vertices, resulting in a loss of accuracy. This instantaneous accuracy loss is essentially traded for long time resolution. Once triggered, the remesh procedure is global, redefining every surface vertex and triangle.

The remeshing trigger implemented is based on the percentage of triangle edges with lengths not suitably matched with the local curvature of the surface (i.e., too short or too long, as detailed in $§ 4.3)$. We define the length ratio of an edge as its length divided by the length scale necessary to capture the local curvature (or the inverse, whichever is larger). Edges with a length ratio greater than a specified threshold $t_{L R}$ are deemed non-conforming. A remesh is triggered if the percentage of non-conforming edges is greater than a second specified threshold $t_{N C}$. Chapter 4 
gives details about the remeshing procedure and its relation to curvature of the surface. $\S 7.3$ shows how the accuracy of calculations is affected by different choices of the remeshing parameters.

\subsection{Pseudocode}

At this point it is informative to give the general outline of the entire program:

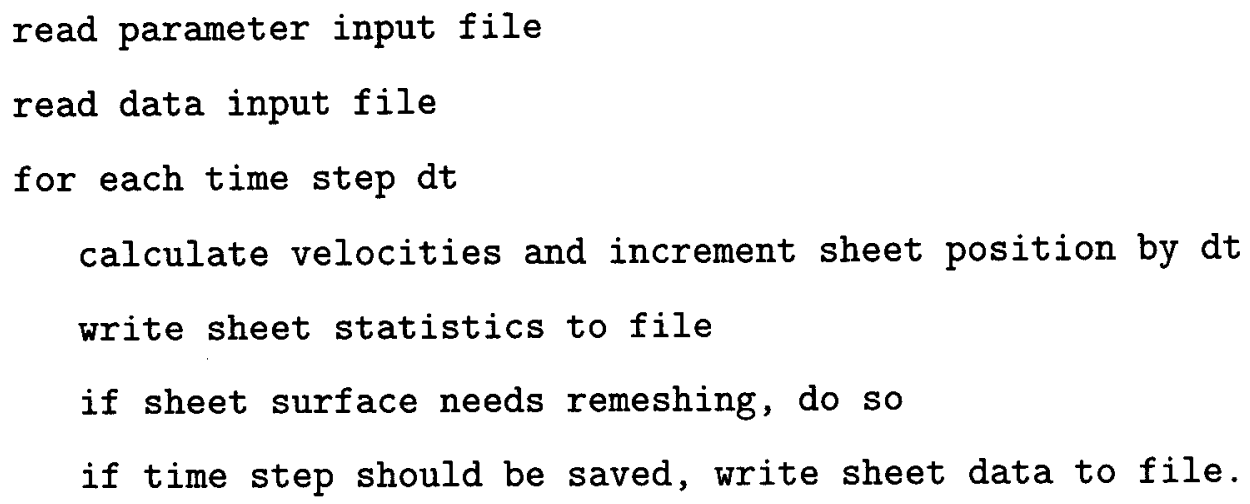

Clearly the calculate velocities and remeshing sections are the most complicated, and are discussed thoroughly in several of the remaining chapters.

\subsection{Computational Hardware}

Almost all computation was done on two or four processor Intel machines, running either Solaris or Linux, with Pentium Pro or Pentium II processors. Some runs were also done on a four processor (R8000) SGI. The hardware limitations of memory, including disk space and RAM, were never reached - computations were always CPU bound. 

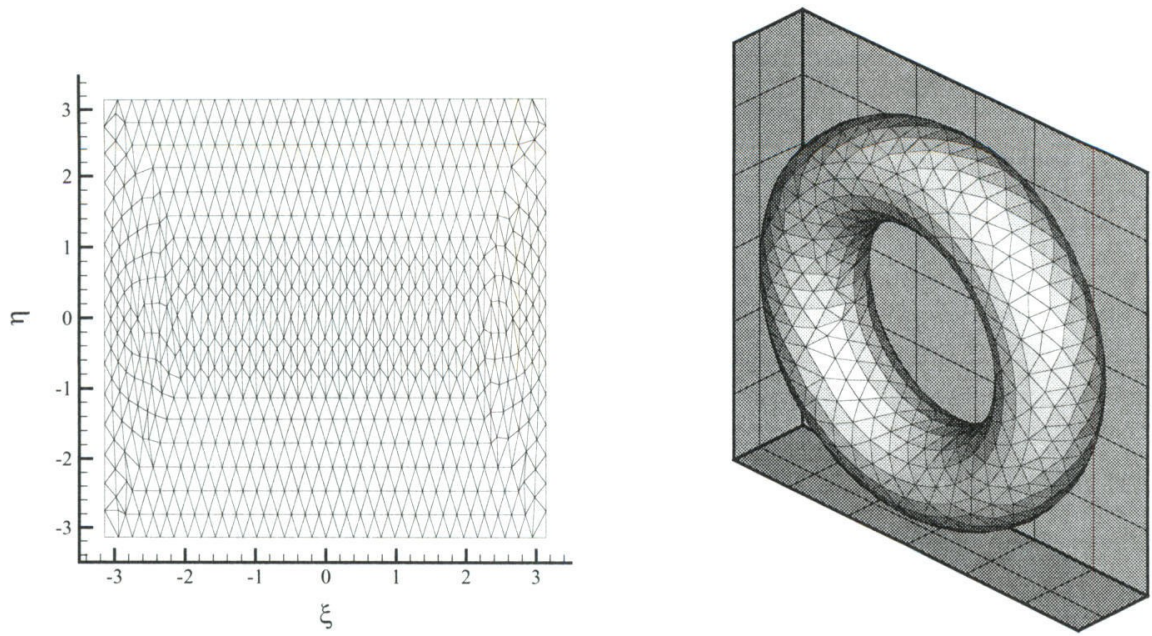

(a)
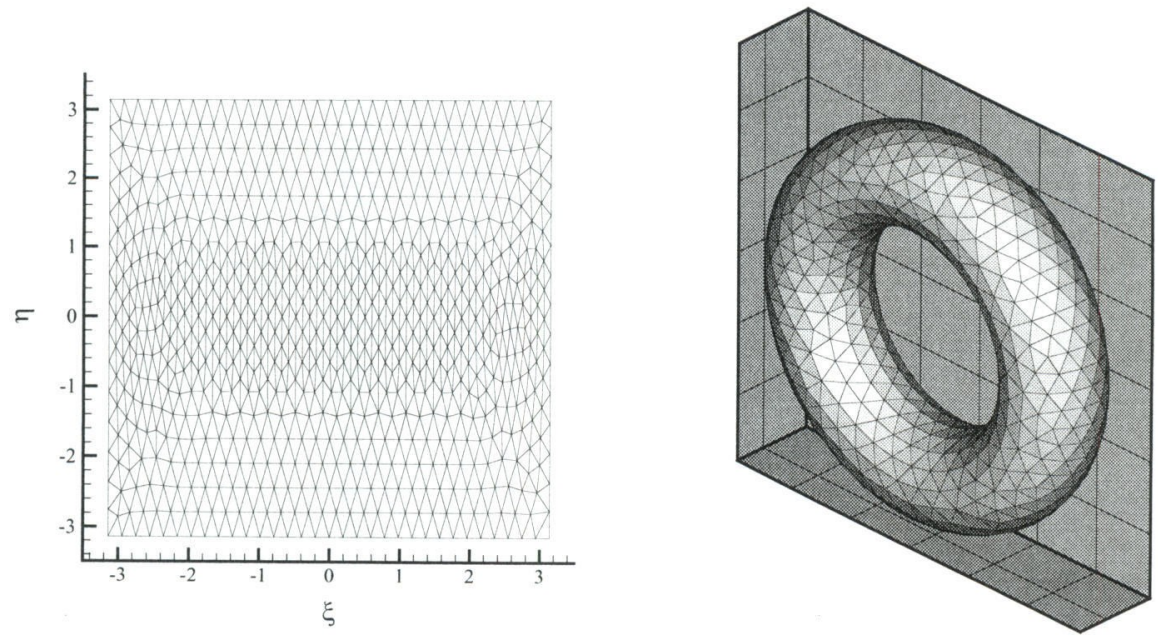

(b)

Figure 3.1: (a) Raw and (b) post-processed parameter and corresponding physical space for a torus. 


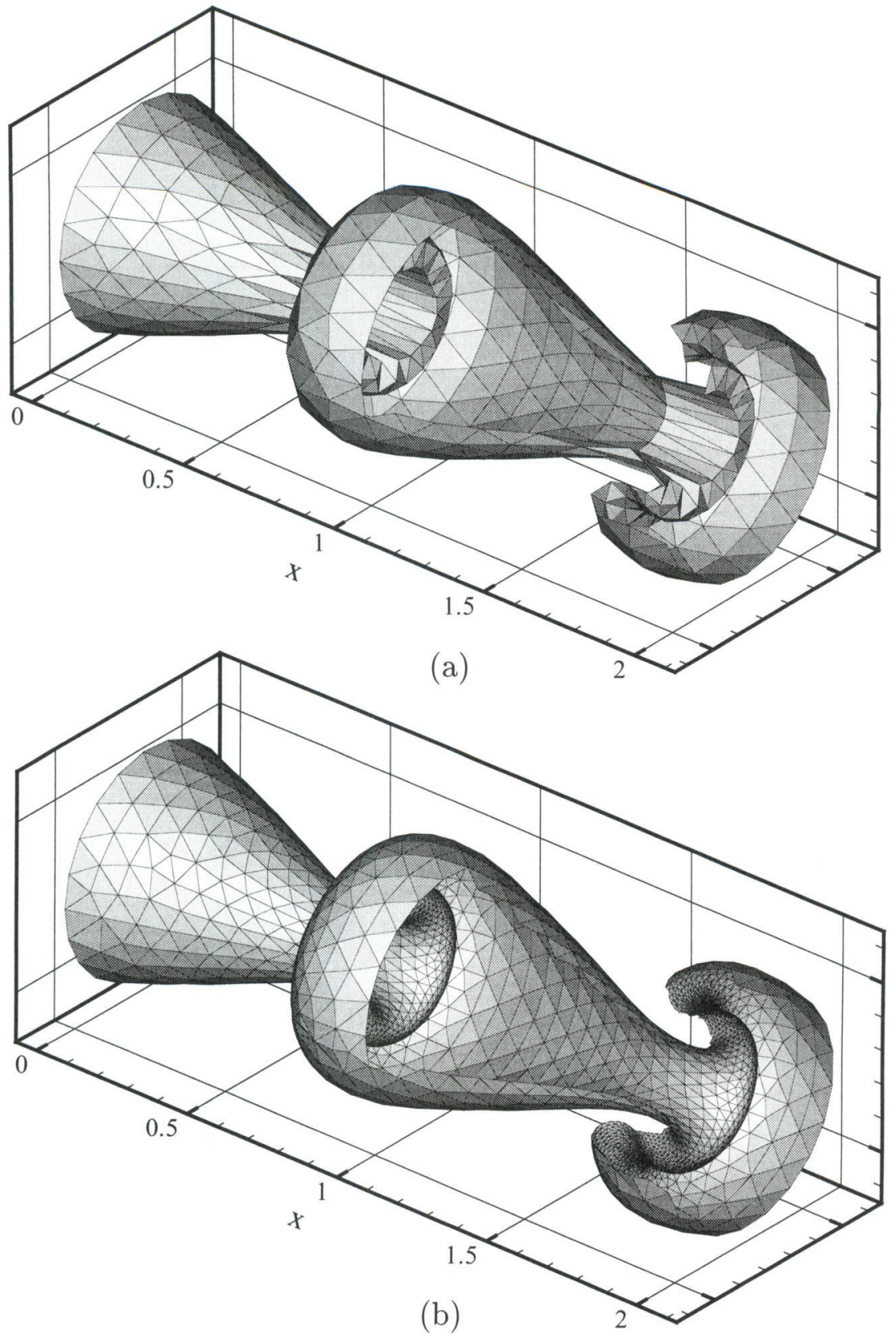

Figure 3.2: Demonstration via mesh skeletons of the need for mesh refinement. Lagrangian deformation of material elements in (a) reveals a loss of resolution with a fixed mesh (600 triangles). With the same initial condition, timely remeshing in (b) allows for an accurate representation of the sheet (5000 triangles). 


\section{Chapter 4 Mesh Generation}

\subsection{Advancing Front}

The advancing front technique is a powerful method for creating a mesh of triangles conforming to user specified size, stretching and boundary constraints [33, 43]. In a general sense, closed directed boundaries are given as input such that the right facing normal consistently points inside the domain. Nodes are then placed along these boundaries (fronts) at a specified, and possibly varying, spacing depending on the surface being triangulated. Each edge of the front (between nodes) is considered in turn as one side of a new triangle, and the ideal location of the triangle's third vertex is calculated following the given size and stretching criteria (described below). The front is adjusted as triangles are generated so as to close in on itself. This particular advancing front implementation fills a square region in parameter space $(\Sigma)$ with specifically sized and oriented triangles such that their mapping into physical space creates the desired surface. Additionally, the physical space triangles are designed to be nearly equilateral and have their area vary proportionally with the inverse of the total surface curvature squared.

\subsection{Density Function}

We define a target triangle density function $f(\xi, \eta)$ as

$$
f=\kappa_{T}^{2}
$$

where $f$ represents the desired number of triangles per unit area in physical space, and $\kappa_{T}^{2}$ is a conservative estimation of the maximum principal curvature. This type of curvature based scaling, e.g., keeps the same number of triangles on a sphere, 
independent of its radius. Bounds are placed on the density function in order to (1) inhibit excessively large triangles in relation to the regularization parameter $\sigma$, and (2) fix an upper bound on the possible number of triangles generated.

\subsection{Triangle Size Scaling}

Given the above density function, it is possible to write an approximate integral relation between the total number of triangles $N$ and the triangle density as

$$
N \cong R \iint_{S} f d S=R \iint_{\Sigma} f J d \Sigma,
$$

where $N$ is the number of triangles associated with the (dimensionless) resolution factor $R$, density function $f$, and mapping Jacobian $J$. For any given surface, $f$ and $J$ can be calculated. The desired resolution is then determined by the specification of $R . R$ is held constant as the sheet evolves, thereby fixing the local relation between density $f$ and triangle area $A$. In fact, under the assumption that triangles are equilateral in physical space with side length $\Delta X$, the local triangle area must be

$$
\frac{\sqrt{3}}{4} \Delta X^{2}=\frac{1}{R f}
$$

and the local physical space length scale is thus

$$
\Delta X=\frac{2}{3^{1 / 4}} \frac{1}{\sqrt{R f}} \cong \frac{1.52}{\sqrt{R f}}
$$

The "resolution" of a surface discretized with this procedure is best characterized with $R$, as $R$, not $N$, gives a better basis for comparison of different surface mesh

resolutions. As an example, Eq. 4.2 carried out for a sphere gives $N=\frac{4 \pi}{3} R$ - the number of triangles $N$ depends only on the resolution $R$, not the size of the sphere. Of course the typical meaning of resolution is a grid length scale like $\Delta X$. For surfaces with little curvature this is appropriate, but when there is high deformation, $\Delta X$ can vary significantly across the sheet, and loses meaning as a global grid characteristic. 


\subsection{Stretching Directions}

To first order the mapping $\mathbf{X}$ can be represented in parameter space as a scaling and rotation of the $(\xi, \eta)$ axis. Thus one can imagine an alternate coordinate system $(\tilde{\Sigma})$ in parameter space which locally has the same topology as the sheet surface. Equilateral triangles defined in this alternate coordinate system $(\tilde{\Sigma})$ will thus be equilateral (to first order) in physical space. $\tilde{\Sigma}$ is defined from a first order expression of Eq. 2.3:

$$
\Delta X=\Delta \xi \sqrt{E \cos ^{2} \theta+F \sin 2 \theta+G \sin ^{2} \theta}
$$

where $\Delta \xi$ is a short segment in $\Sigma$, inclined at an angle $\theta$ relative to the $\xi$ axis, and $\Delta X$ is the corresponding mapped length. The two extreme values of $\Delta X$ are obtained when $\theta$ satisfies

$$
\tan 2 \theta=\frac{2 F}{E-G}
$$

The two corresponding $\theta$ values $\left(\theta_{1}, \theta_{2}\right)$ designate the axes of $\tilde{\Sigma}$. When $E=G$ there is no stretching and $\tilde{\Sigma}$ is equivalent to $\Sigma$. The scaling along each axis in $\tilde{\Sigma}$ is

$$
s_{i}=\frac{\Delta X}{\Delta \xi}=\sqrt{E \cos ^{2} \theta_{i}+F \sin 2 \theta_{i}+G \sin ^{2} \theta_{i}}
$$

for $i=1,2$.

\subsection{Boundary Node Placement}

The advancing front technique begins by placing nodes along the perimeter of the domain, creating the initial "front." The first node is placed in one corner, then while marching along the perimeter, nodes are placed at a density corresponding to $f$. The incremental distance $(\Delta \xi)$ to the next node is determined by evaluating Eq. 4.3 for the current desired physical space length scale $\Delta X$. Eq. 4.4 is then used with the 
current parameter space direction $(\theta)$ to calculate $\Delta \xi$. When subsequent parameter space corners are not hit exactly (typically the case), the position of each node on the current boundary is scaled by a small amount such that they all fit exactly.

\subsection{Interior Ideal Node Placement}

The most common advancing front step involves examining a directional segment in $\Sigma$ between two nodes in order to decide where a third node should be placed such that a triangle is created with nearly equilateral mapping. To this end, the segment is transformed to $\tilde{\Sigma}$, using the midpoint of the segment to define $\left(\theta_{1}, \theta_{2}\right)$ and $\left(s_{1}, s_{2}\right)$. A new node is placed in $\tilde{\Sigma}$ such that the triangle formed with it and the segment is nearly equilateral, with side length $\Delta X$ from Eq. 4.3. Finally the new node is transformed back to $\Sigma$, its location deemed ideal.

\subsection{Implementation}

Implementation of the advancing front method begins by defining boundary nodes of the parameter space domain $(\xi= \pm \pi, \eta= \pm \pi)$. Since the domain is periodic (the mesh in $\Sigma$ is always periodic, independent of the surface type being represented: SWP0, 1, or 2), only two sides of the boundary are defined with the technique described previously; the other two sides are redundant copies. The segments between each node are the edges of future triangles. A single front is defined as the clockwise traversal of the segments between nodes. Each segment is examined in turn and a new triangle created, until the front has closed in on itself, and no segments remain. Triangles are generated for each segment by first calculating the corresponding ideal node location, as described in the previous section. A new node is inserted at that location only if feasible, as in Fig. 4.1(b); otherwise, as in Fig. 4.1(a), an existing node is used to create a new triangle and advance the front. Fig. 4.2 shows various stages during an example mesh creation. Note that the front may pinch itself off, generating two child fronts, as seen in Fig. 4.2(d). The initial mesh as well as subse- 


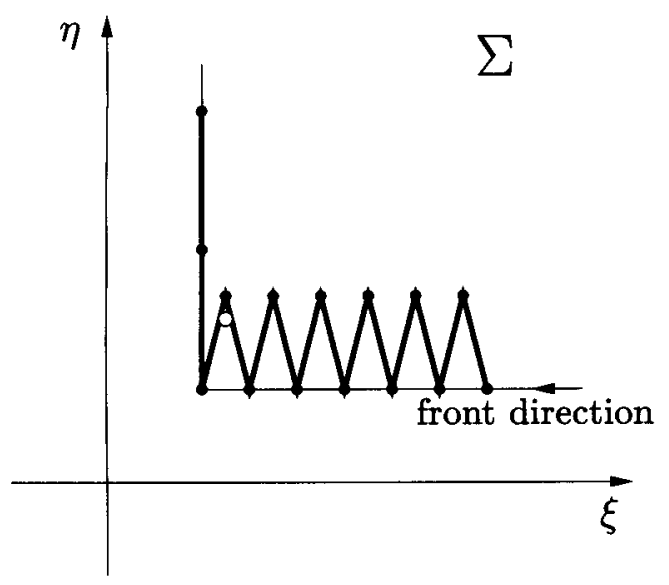

(a)

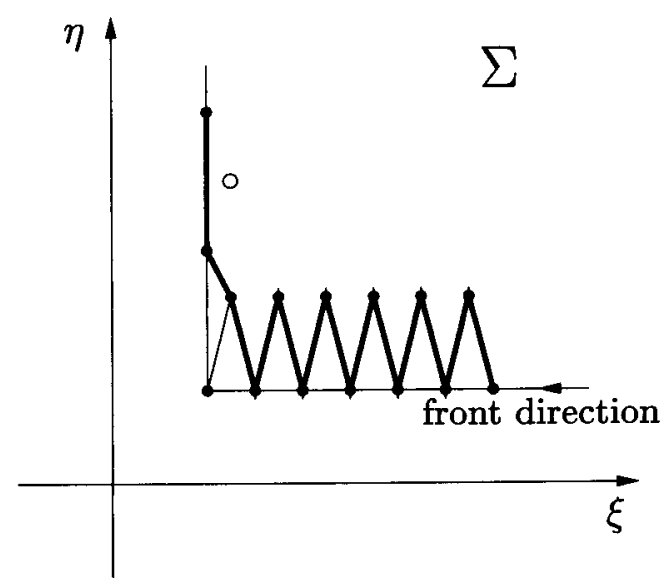

(b)

Figure 4.1: Example ideal node placement and usage, active edge shown in red. In (a), an existing node (filled circles) in the front is close enough to the ideal position (open circle) to be substituted. In (b) there are no nearby existing nodes - a new node will be inserted at the ideal position and the front updated.

quent re-meshes are generated with this procedure. While generating and refining a new mesh, the old existing one remains active in order to evaluate the density $f$ and stretching directions, and fill the new mesh with $\mathbf{X}$. The mesh generation process takes $O\left(N^{3 / 2}\right)$ time, as a search through $O\left(N^{1 / 2}\right)$ triangles (those with edges in the current front) is necessary for each of the $N$ triangles generated.

\subsection{Mesh Post-Processing}

The mesh generated by this advancing front algorithm is not ideal, as can be seen with a keen eye in the physical space representation in Fig. 3.1(a). Whenever two fronts collide, closing a region, ideal nodes are typically not selected and the corresponding triangles in physical space are far from equilateral. But a completed mesh can be improved with a variety of fast operations. Laplace smoothing [11] is the name given to a mesh process in which every node is moved to the center of gravity of its neighboring triangles. In this application, we wish to maintain equilateral triangles in physical space, so vertex coordinates in parameter space are moved to the physical space area-weighted average of the neighboring triangles. The method may 
be applied repetitively, but a few $(<5)$ iterations result in significant improvement. Mesh relaxation $[9,16]$ attempts to push the number of triangles adjacent to each node (the degree $d$ ) towards six. To this end, every edge between two triangles is considered in turn. The edge is swapped if (a) geometrically feasible (see Fig. 4.3) and (b) the regularity index, $\bar{d}=\sum_{i=1}^{4}\left(d_{i}-6\right)^{2}$, improves beyond a threshold. The summation index $i$ runs over the four vertices needed to define the edge under consideration and the two adjacent triangles. When $\bar{d}$ is zero, the four vertices have ideal connectivity with respect to mesh relaxation. The third and final mesh improvement technique swaps edges based on the desire to line them up with valleys and ridges of the surface. Define the edge-center-distance (ECD) as the distance in physical space from the mapping of the edge midpoint, to the middle of the physical space edge (see Fig. 4.4). Each edge is considered in turn, and is swapped if (a) geometrically feasible and (b) the swapped edge's ECD is some degree less than the ECD of the current edge. Fig. 3.1(b) shows the improvement made after two passes of Laplace smoothing followed one pass through ECD swapping and five passes of relaxation (thresholds from 11 to 1 by 2), followed finally by two more passes of Laplace smoothing. Post processing effectiveness is monitored by mesh statistics such as average skewness ( $\max / \min$ triangle lengths) and the distribution of $\bar{d}$. 


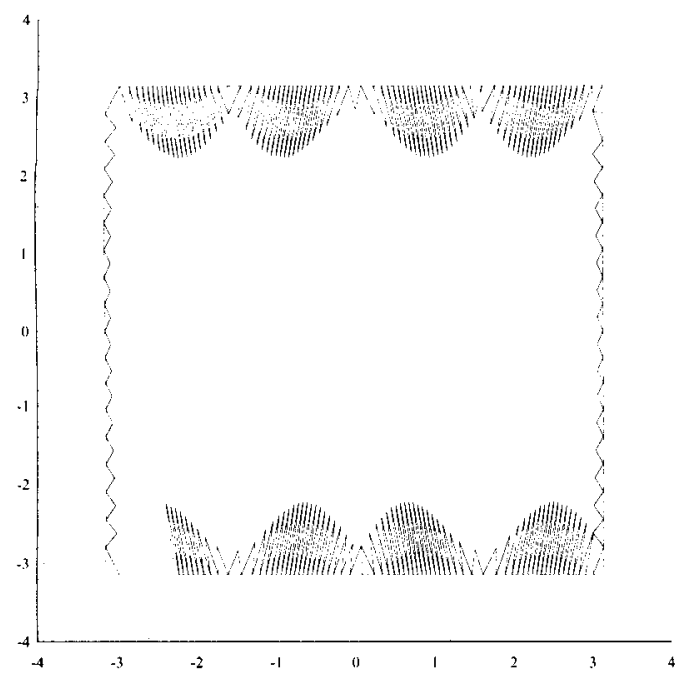

(a)

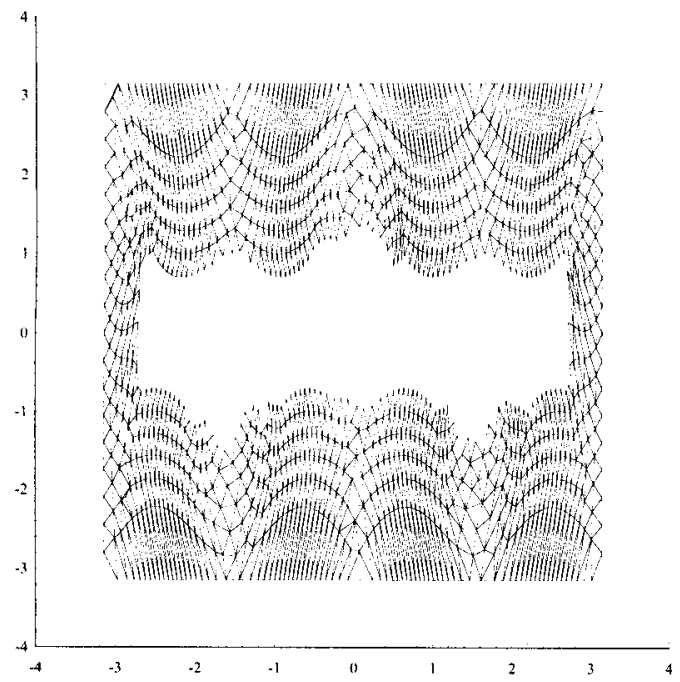

(c)

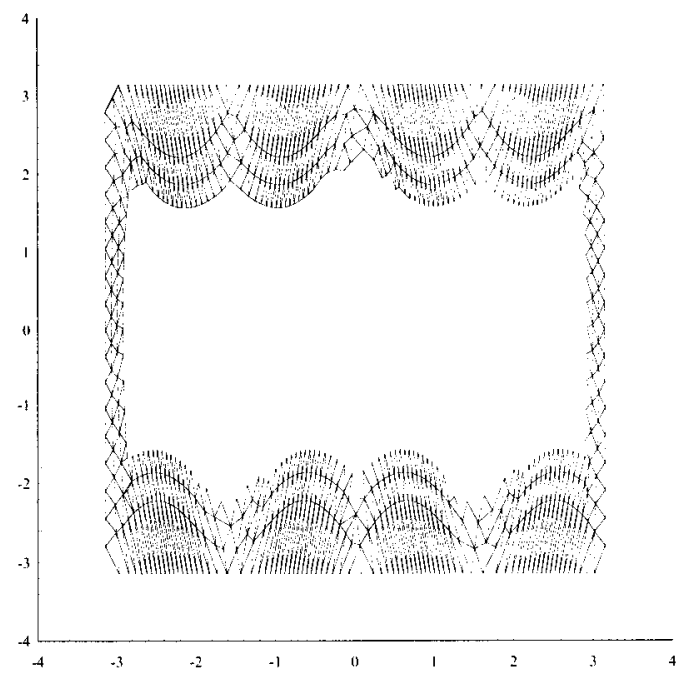

(b)

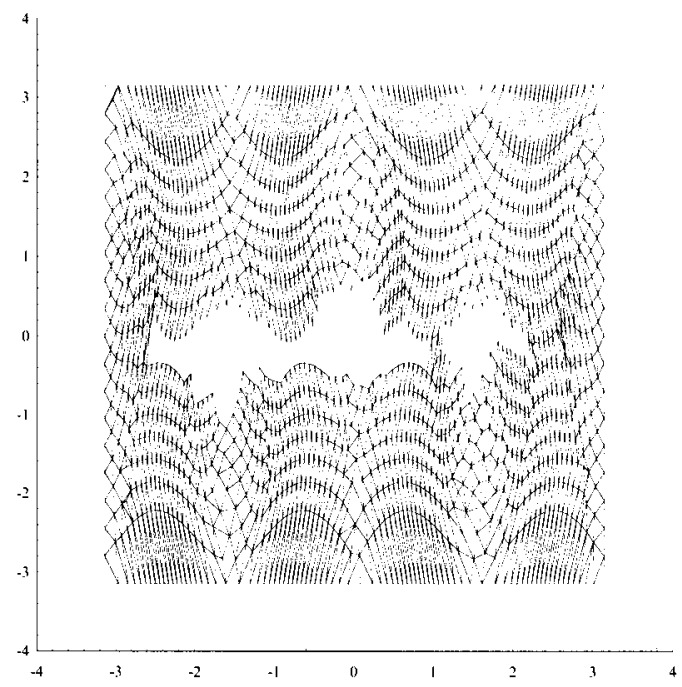

(d)

Figure 4.2: Advancing front in parameter space $\Sigma$ with 185, 1000, 2000, and 2650 triangles. Note how in (d) the front has pinched itself closed, generating two child fronts.

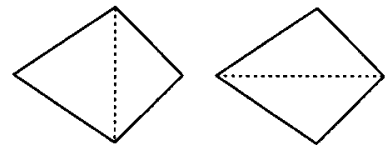

(a)

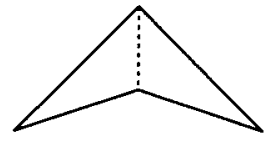

(b)

Figure 4.3: Dashed lines represent (a) swapped and (b) un-swappable edges. 


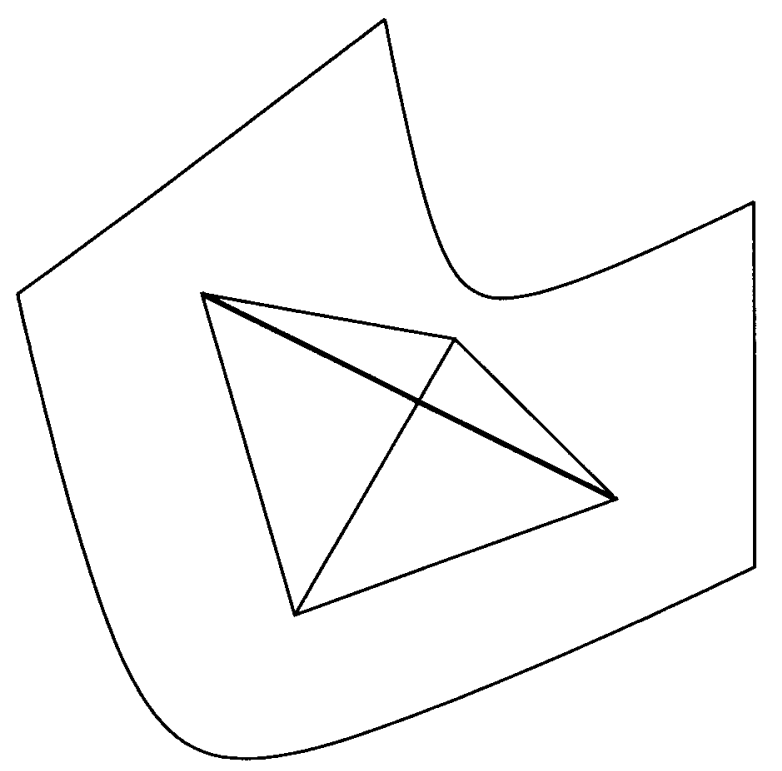

Figure 4.4: The two black triangles conform nicely to the local surface topology. The edge in the valley is considered for swapping with the alternate red edge. The ECD of the black edge is approximately zero, as its midpoint is essentially on the surface, but the distance of the midpoint of the red edge to the surface is significantly more. Thus the black edge will not be swapped for the red. 


\section{Chapter 5 Improved Velocity Evaluation}

\subsection{Introduction}

The overall algorithmic performance of a computer code, in terms of memory and time required, is often a matter of keen interest for the obvious reason of generating desired results as quickly as possible on a given hardware platform, with a specified error tolerance. This chapter discusses reducing the order of a Biot-Savart integrator from $O\left(N^{2}\right)$ to $O\left(N^{3 / 2}\right)$ with a multi-pole expansion technique. This technique is based on a simple Taylor expansion of the Biot-Savart kernel, but is powerful enough to reduce the order of velocity evaluation, in stages of implementation complexity, to $O\left(N^{3 / 2}\right), O(N \log N)$, and even $O(N)$. These ideas are not new and were in fact popularized by the thesis of Greengard [19]. However, the expansions presented here are different than the spherical harmonics used by Greengard, a necessity of the modified Biot-Savart kernel [32].

The advantage of the multi-pole expansion is that patches of vorticity (in this case neighboring triangles) can contribute to the velocity at a target point in groups, rather than individually. Furthermore, a large portion of each groups' influence can be pre-calculated for all target points. To determine the total velocity at a target point, contributions from nearby triangles are added individually, while contributions from distant triangles, grouped together in geometric bounding boxes, are taken as lump sums. An $O\left(N^{3 / 2}\right)$ method is achieved if each box has specific side dimension $L_{B}$ (defined below) and there is no hierarchy with boxes containing other boxes (as an example see Fig. 5.1). If, however, triangles are encased by boxes in a hierarchical subdivision (e.g., an oct-tree representation), a full velocity evaluation only takes $O(N \log N)$ time [32]. The final speed-up, to an $O(N)$ method, is realized by allowing boxes to influence other boxes (and hence many target points at once), rather than single target points. All three methods suffer from needing to re-calculate the box 
locations and generic influences prior to each full velocity evaluation (and there may be multiple velocity evaluations per time step, depending on the time integration technique).

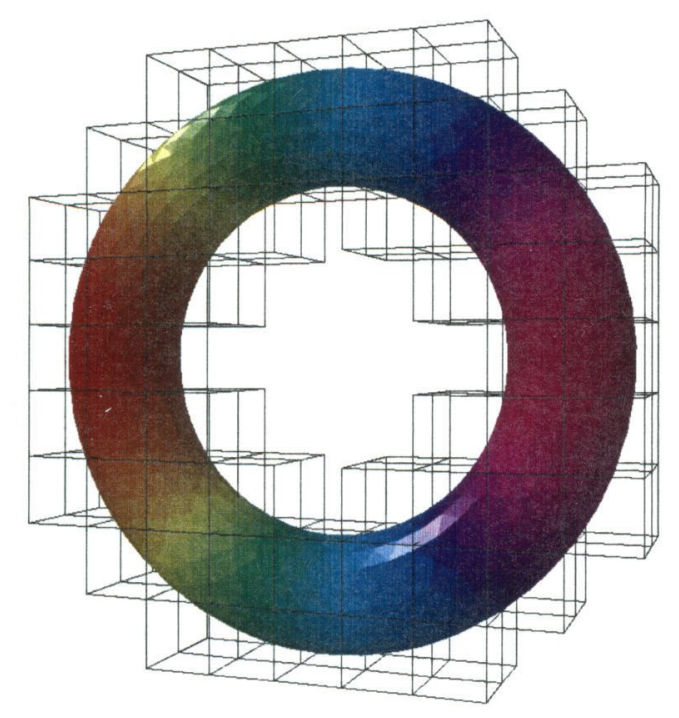

Figure 5.1: An example triangulated sheet encased in a non-hierarchical subdivision of boxes, each with side length $L_{B}$.

\subsection{Velocity Evaluation}

First, the optimum side length $\left(L_{B}\right)$ of each box is calculated as described in detail below. Then the triangulated vortex sheet is divided into cubes of side $L_{B}$, each containing a group of neighboring triangles. Subsequently, the influence (also described below) of each box is calculated and stored. Finally, the velocity at each vertex (target point) is calculated and time integration proceeds. (An example targetbox geometry is shown in Fig. 5.4.)

In order to determine when to use the target-box interaction (as opposed to target-triangle), we introduce the notion of an error tolerance, $E_{T O L}$. When the target point is "close" to the box in some sense (also described below), the error incurred with the multi-pole expansion is greater than when the box is far away. Thus, based on the desired maximum absolute error per target-box interaction $E_{T O L}$, 
a critical distance $d_{c r i t}$ is defined for each box of triangles, specifying the minimum distance between an acceptable target-box interaction.

\subsection{Scaling Arguments}

At any one time, the triangulated vortex sheet with $N$ triangles is encased by $B$ boxes, with an average of $T$ triangles per box. The velocity at a target point is determined by the influence of $\alpha B$ box interactions, plus $(1-\alpha) B T$ triangle interactions, where $\alpha$ denotes the average fraction of boxes used. Thus, the total time required to calculate the velocity at every vertex of the sheet (a full velocity evaluation) scales as

$$
t_{\mathrm{FVE}} \propto N[\alpha B+(1-\alpha) B T]
$$

Consistent with the definitions above, we must have $N=B T$. To achieve an $O\left(N^{3 / 2}\right)$ method, we also need $(1-\alpha) \propto N^{-1 / 2}$, as seen by the second term inside the square brackets of Eq. 5.1. It follows that both $B \propto N^{1 / 2}$ and $T \propto N^{1 / 2}$. The number of boxes (cubes) $B$ is determined by their side length $L_{B}$ and the amount of space filled by the vortex sheet. One may estimate $L_{B}$ by assuming the sheet to be locally planar inside each box, so that the total surface area $A$ may be approximated as $A \approx B L_{B}^{2}$ which leads to

$$
L_{B} \propto \sqrt{A / \sqrt{N}}
$$

To test all the scaling arguments discussed above, we consider a test case fixed in time. Varying only $N$, we examine the behavior of $t_{\mathrm{FVE}}$, the execution time. The results are summarized in Fig. 5.2, comparing two values of the error tolerance $E_{T O L}$ with the $O\left(N^{2}\right)$ method. With $E_{T O L}>0$ it is clear that we are quite close to an $O\left(N^{3 / 2}\right)$ velocity evaluation technique.

Unfortunately, in a typical simulation with adaptive refinement, triangles in different regions of the sheet generally develop significantly different length scales (see 


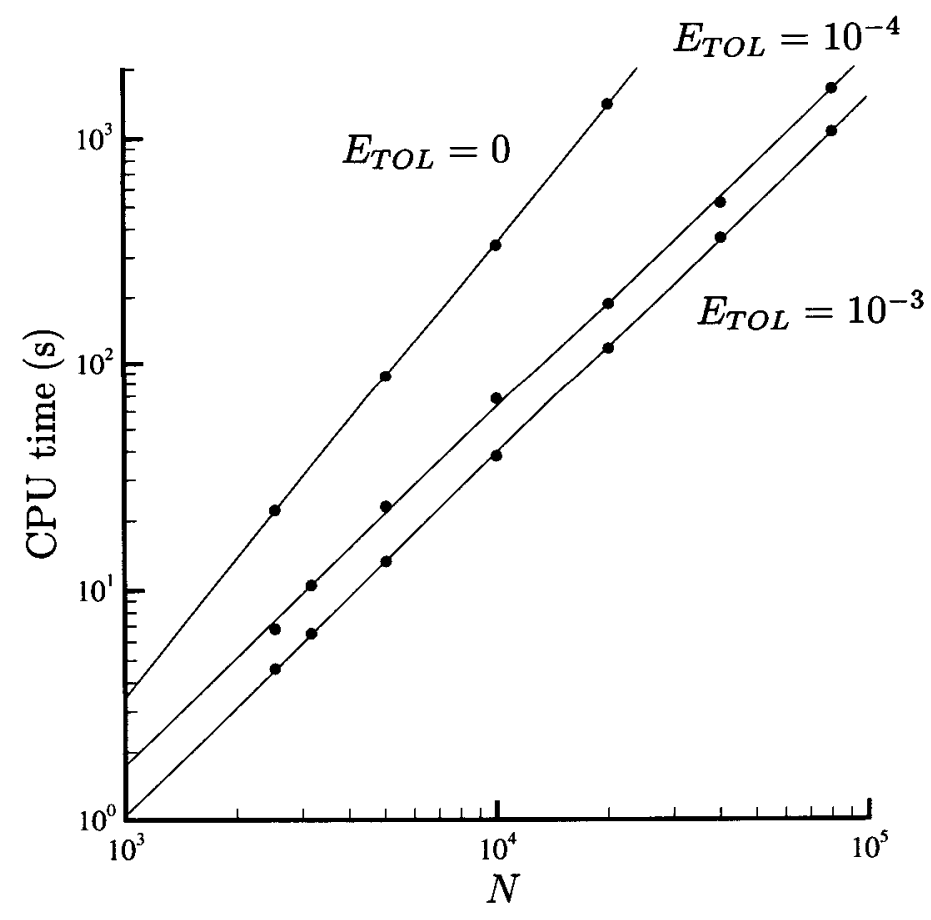

Figure 5.2: Duration of one time step (single processor) versus the number of triangles, $N$. $O\left(N^{2}\right)$ method in red; $O\left(N^{3 / 2}\right)$ method in green and blue. Solid lines have slopes $2.0,1.55$ and 1.57 respectively. With $E_{T O L}=10^{-3}$, speed is increased by a factor of 10 near $N=20,000$.

Chapter 8), leading to a wide variation in the number of triangles per box. In this situation, the desired scaling $(1-\alpha) \propto N^{-1 / 2}$ fails and the method reverts to $O\left(N^{2}\right)$. It is, however, still considerably faster than the original $O\left(N^{2}\right)$ method. Fig. 5.3 shows timing data from an actual simulation. The transition from $O\left(N^{3 / 2}\right)$ to $O\left(N^{2}\right)$ unfortunately occurs rather quickly, but clearly the method is significantly faster than the direct $O\left(N^{2}\right)$ method. Note the high red data point in the scatter for each mesh size $N$ represents the extra time taken for the automatic mesh refinement. 


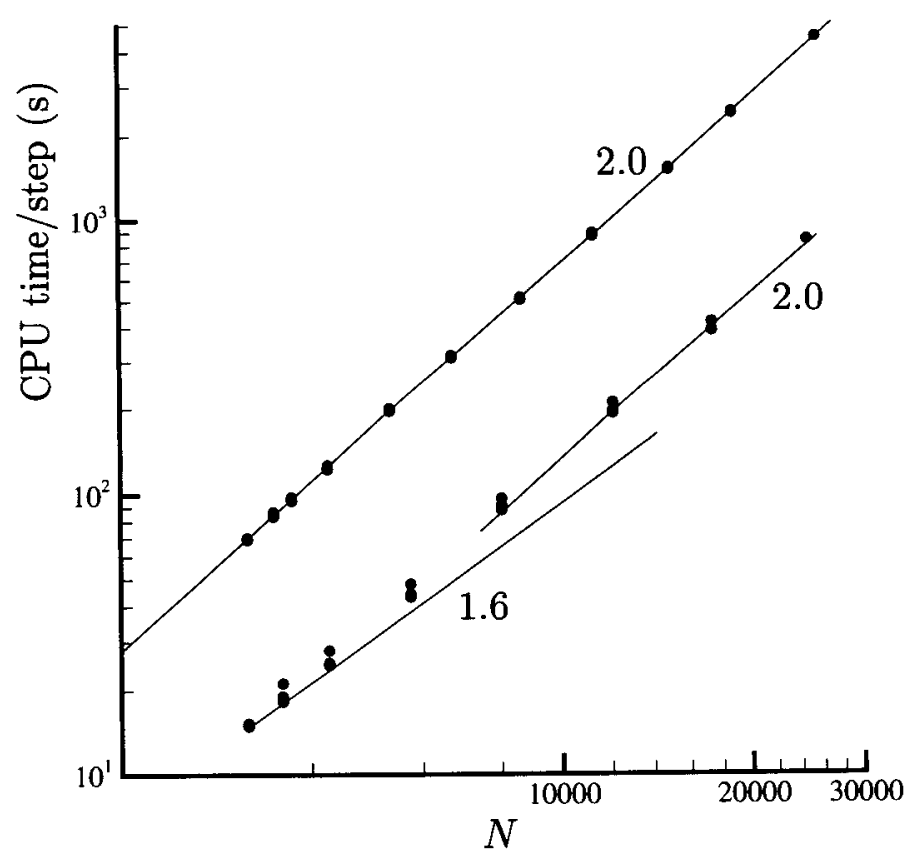

Figure 5.3: CPU time per time step plotted against the number of current triangles in the mesh $(N)$ for an example simulation (see Appendix 7.1). Direct $O\left(N^{2}\right)$ method in green, $O\left(N^{3 / 2}\right)$ method in red. Time steps in the simulation implicitly travel from left to right, $N$ increasing as the sheet evolves. Numbers next to lines specify the slope.

\subsection{Expansion, Error Bound, and Critical Distance}

The regularized Biot-Savart integral Eq. 2.22 may be written (non-parameterized) as

$$
\begin{aligned}
\mathbf{u}^{V}(\boldsymbol{x}) & =\frac{1}{4 \pi} \int_{V} \frac{\boldsymbol{\omega}\left(\boldsymbol{x}^{\prime}\right) \times\left(\boldsymbol{x}-\boldsymbol{x}^{\prime}\right)}{\left[\left|\boldsymbol{x}-\boldsymbol{x}^{\prime}\right|^{2}+\sigma^{2}\right]^{3 / 2}} d^{3} \boldsymbol{x}^{\prime} \\
& =\frac{1}{4 \pi} \int_{V} \boldsymbol{\omega}^{\prime} \times \mathbf{K} d^{3} \boldsymbol{x}^{\prime}
\end{aligned}
$$

where

$$
\mathbf{K}=\frac{\boldsymbol{x}-\boldsymbol{x}^{\prime}}{\mathbf{D}_{\sigma}^{3}}, \quad \mathbf{D}_{\sigma}=\left[\left|\boldsymbol{x}-\boldsymbol{x}^{\prime}\right|^{2}+\sigma^{2}\right]^{3 / 2}
$$


and $\mathrm{V}$ now represents the volume of a box containing a collection of neighboring

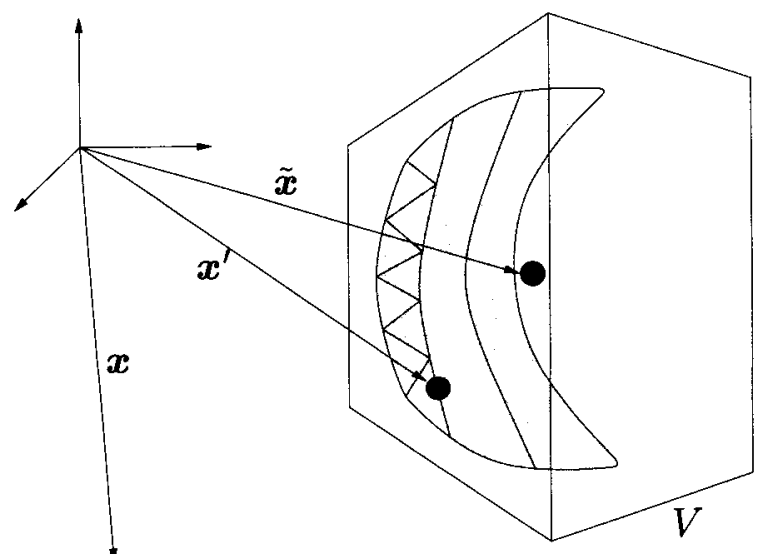

Target point

Figure 5.4: Patch of triangulated vortex sheet inside box with volume $V$. $\tilde{\boldsymbol{x}}$ is the box centroid and $\boldsymbol{x}^{\prime}$ is a point on the sheet surface.

triangles (as shown in Fig. 5.4). To derive the multi-pole expansion, we substitute the Taylor series of $\mathbf{K}$ about $\boldsymbol{x}^{\prime}=\tilde{\boldsymbol{x}}$ into Eq. 5.4. $\tilde{\boldsymbol{x}}$ represents a point inside the box, henceforth the box centroid. Switching to index notation and skipping intermediate steps, we have

$$
\begin{aligned}
u_{i}^{V}(\boldsymbol{x})= & \frac{1}{4 \pi} \epsilon_{i l m} \int_{V} \omega_{l}^{\prime} K_{m} d^{3} \boldsymbol{x}^{\prime} \\
= & \frac{1}{4 \pi} \epsilon_{i l m} \tilde{D}_{\sigma}^{-3}\left[\hat{x}_{m} M_{l}^{0}+3 \tilde{D}_{\sigma}^{-2} \hat{x}_{j} \hat{x}_{m} M_{l j}^{1}-M_{l m}^{1}\right. \\
& \left.\quad+\frac{15}{2} \tilde{D}_{\sigma}^{-4} \hat{x}_{j} \hat{x}_{k} \hat{x}_{m} M_{l j k}^{2}-\frac{3}{2} \tilde{D}_{\sigma}^{-2} \hat{x}_{m} M_{l j j}^{2}-3 \tilde{D}_{\sigma}^{-2} \hat{x}_{j} M_{l j m}^{2}+\cdots\right]
\end{aligned}
$$

where $M_{i}^{0}, M_{i j}^{1}, M_{i k j}^{2}$ represent the zero, first and second moments of vorticity respectively, i.e.,

$$
\begin{aligned}
M_{i}^{0} & =\int_{V} \omega_{i}^{\prime} d^{3} \boldsymbol{x}^{\prime} \\
M_{i j}^{1} & =\int_{V} \omega_{i}^{\prime} \hat{x}_{j}^{\prime} d^{3} \boldsymbol{x}^{\prime} \\
M_{i j k}^{2} & =\int_{V} \omega_{i}^{\prime} \hat{x}_{j}^{\prime} \hat{x}_{k}^{\prime} d^{3} \boldsymbol{x}^{\prime}
\end{aligned}
$$


and

$$
\begin{aligned}
\hat{x}_{i} & =(x-\tilde{x})_{i} \\
\hat{x}_{i}^{\prime} & =\left(x^{\prime}-\tilde{x}\right)_{i} \\
\tilde{D}_{\sigma} & =\left[|\boldsymbol{x}-\tilde{\boldsymbol{x}}|^{2}+\sigma^{2}\right]^{3 / 2} .
\end{aligned}
$$

In this way, the generic influence of each box is represented by the moments of vorticity, $M^{i}$. An error bound [53] for the above series expansion with $\sigma=0$, taken to second order as in Eq. 5.7, is

$$
E=\frac{B_{0}}{d^{2}} \frac{1}{(1-b / d)^{2}}\left(\frac{b}{d}\right)^{3}\left[4 \frac{B_{2}}{B_{0} b^{2}}-3\left(\frac{B_{2}}{B_{0} b^{2}}\right)^{2}\left(\frac{b}{d}\right)\right],
$$

where

$$
\begin{aligned}
& B_{0}=\int_{V}\|\boldsymbol{\omega}\| d^{3} \boldsymbol{x} \\
& B_{2}=\int_{V}\|\boldsymbol{\omega}\|(\boldsymbol{x}-\tilde{\boldsymbol{x}}) \cdot(\boldsymbol{x}-\tilde{\boldsymbol{x}}) d^{3} \boldsymbol{x} .
\end{aligned}
$$

Here $b$ represents the radius of the box, and $d$ is the distance from the box to the target point, as in Fig. 5.5. The first moment $B_{1}$ has been eliminated by a more aggressive expression involving $B_{2}$. In practice, for each box $B_{0}, B_{2}$, and b are known; $E_{T O L}$ is then specified in order to calculate $d_{c r i t}$ for the box, via iteration of Eq. 5.14. This error bound is considered appropriate to use even for $\sigma>0$ because we always have $d_{\text {crit }}$ several times larger than $\sigma$.

Finally, we recognize that the error bound applies for each target-box interaction. Of practical interest is the overall error incurred for the total velocity at each target point, as a function of $E_{T O L}$. To this end, we examine the PDFs of scaled differences (errors) between an $O\left(N^{3 / 2}\right)$ time step and an $O\left(N^{2}\right)$ time step. The non-dimensional error is defined at each triangle vertex as the difference in position (after one step with a very small $\Delta t$ ) between the $O\left(N^{3 / 2}\right)$ and $O\left(N^{2}\right)$ methods divided by the traveled 


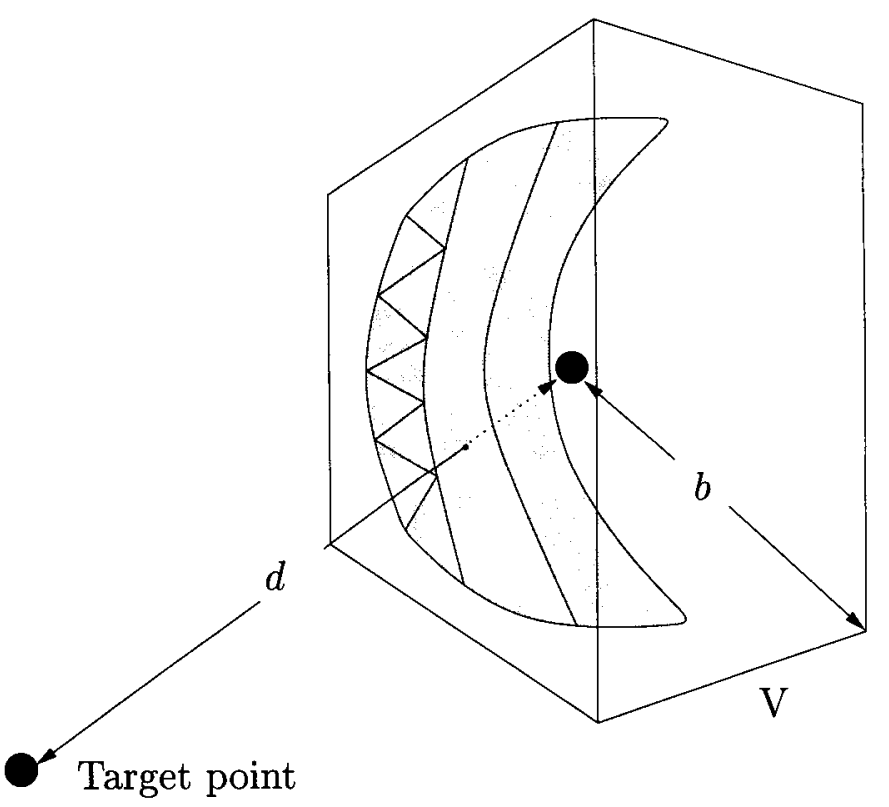

Figure 5.5: Patch of triangulated vortex sheet inside box with volume $V$. Schematic of box-target interaction.

distance of the $O\left(N^{2}\right)$ method. Thus at each vertex we effectively calculate

$$
e=\frac{\left|\boldsymbol{u}_{O\left(N^{3 / 2}\right)}-\boldsymbol{u}_{O\left(N^{2}\right)}\right|}{\left|\boldsymbol{u}_{O\left(N^{2}\right)}\right|}
$$

for which we approximate the PDF $f$, based on a 20 bin sampling of each vertex, as shown in Fig. 5.6. As $E_{T O L}$ drops by factors of ten, the average error does as well, revealing the error bound Eq. 5.14 scales properly with $E_{T O L}$. In Chapter $7, E_{T O L}$ is included in the set of parameters (time step, mesh resolution, etc.) which must be balanced to produce an efficient calculation with a chosen overall error tolerance. 


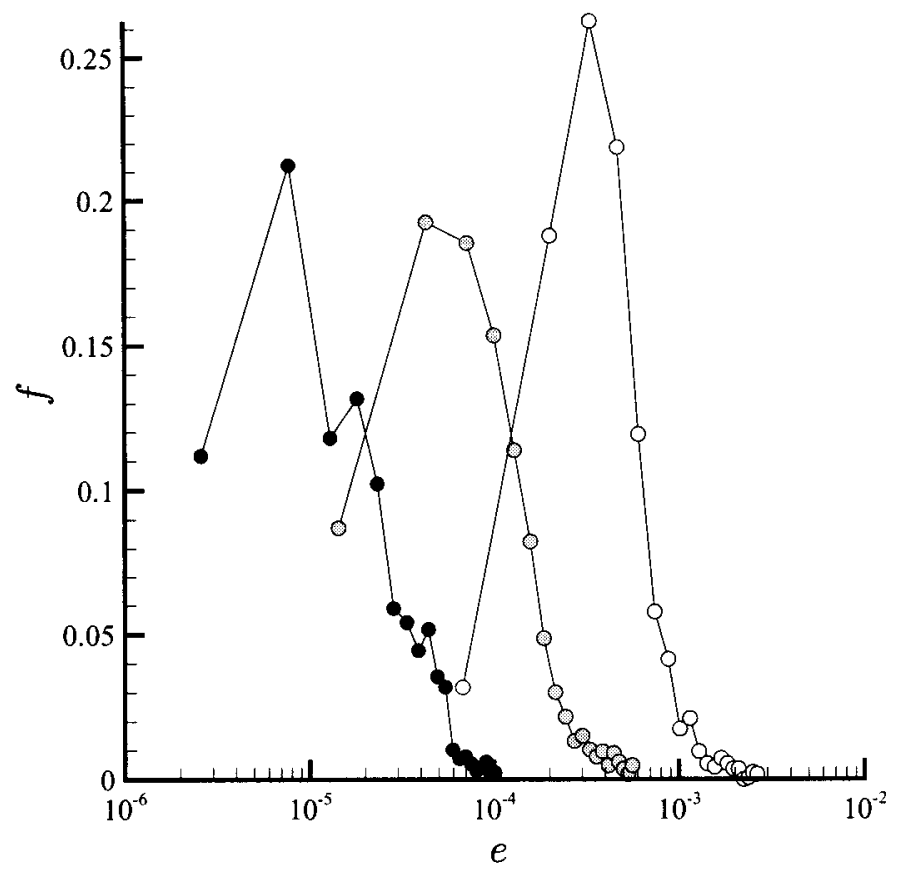

Figure 5.6: PDF of scaled difference between $O\left(N^{3 / 2}\right)$ time step and an $O\left(N^{2}\right)$ time step. $E_{T O L}$ decreases with symbol darkness from $10^{-2}$ to $10^{-3}$ to $10^{-4}$. Average strain (error) drops roughly from $10^{-3}$ to $10^{-4}$ to $10^{-5}$. 


\section{Chapter 6 Periodic Velocity Evaluation}

\subsection{Introduction}

As discussed in $\S 2.4$, an efficient procedure for evaluating the velocity on the surface of a periodic sheet is needed. Two of the sheet types are of interest: SWP1 and SWP2. In essence, it is of course not feasible to evaluate the influence of infinitely many triangles on any one target point. Even with the spatial subdivision techniques discussed in Chapter 5, there would be infinitely many boxes in these periodic sheet types. What is needed is a method to evaluate the influence of the infinite periodic extension of the base period - all at once - in an acceptable amount of time. The $O\left(N^{2}\right)$ technique described in section $\S 2.4$ for the SWP1 surface type accomplishes this goal. However, that method is incompatible with the fast velocity evaluations of the previous chapter. Thus to utilize the faster velocity evaluation method for the SWP1 and SWP2 surface types, the techniques presented in this chapter are used.

The easiest solution is simply to think of the influence of each period on a given target point as a term in an infinite series. This series may be trivially truncated at $p$ terms, say, and the resultant sum used as the velocity. This method of course results in significant errors, depending on the number of terms taken and the convergence properties of the series. A first alternative is to use the Ewald summation technique [6], which involves transforming the aforementioned infinite series into a new series which converges at an exponential rate. The technique presented below is completely different, and follows from previous work by Greengard [19], though none of the details are the same due to the fact that we cannot use the same spherical harmonic multi-pole expansions (this is a consequence of the kernel smoothing via $\sigma)$. The basic idea is to calculate the influence of one full period as a multi-pole expansion. If this expansion can be shifted (algebraically), one can write an expression for the cumulative influence of the multi-pole expansions of all periodic images, 
as a Taylor series about the origin. That is, the influence of all periodic images is combined into a simple Taylor series about the origin.

\subsection{Example}

First, as a simple 2D example, consider a function $\phi(z)$ defined the complex plane, which represents the "influence" (in a Biot-Savart sense) of a square region about the origin (specifically the base period $P_{0}$ - see Fig. 6.1) on an arbitrary target point $z$. This is, in some sense, analogous to a pre-integrated form of the Biot-Savart integral (Eq. 2.13). We want an expression for the "influence" of all distant image periods in $P_{2 \infty}$. We begin by writing the $p^{\text {th }}$ order multi-pole expansion of $\phi$ about the origin as

$$
\phi(z)=\sum_{m=1}^{p} \frac{a_{m}}{z^{m}}
$$

which gives the influence of $P_{0}$ on a sufficiently distant target point $z$. Since each periodic image of $P_{0}$ has the same expansion about its local center, we can also trivially generalize (i.e., translate) the series to give the influence of the square region (with the same dimensions as $P_{0}$ ) about $w$ on the target point $z$ (for $w$ sufficiently far from $z$ ),

$$
\phi_{w}(z)=\sum_{m=1}^{p} \frac{a_{m}}{(z-w)^{m}} .
$$

Now consider $w$ to be sufficiently far away from the origin, but $z$, the target point near the origin. Then Eq. 6.2 may be expressed as a Taylor series in $z$ about the origin via

$$
\phi_{w}(z)=\sum_{n=0}^{p} b_{n} z^{n}+O\left(z^{p+1}\right)
$$




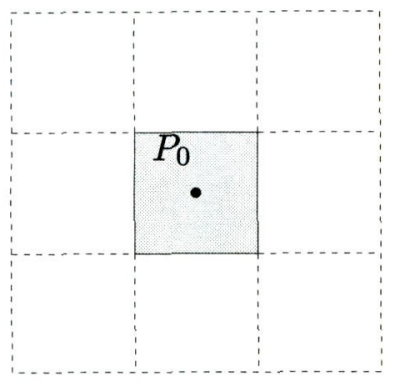

(a)

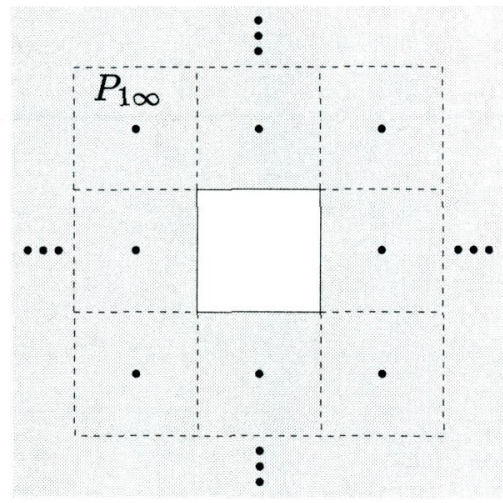

(c)

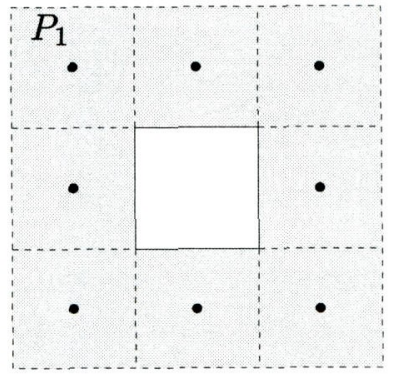

(b)

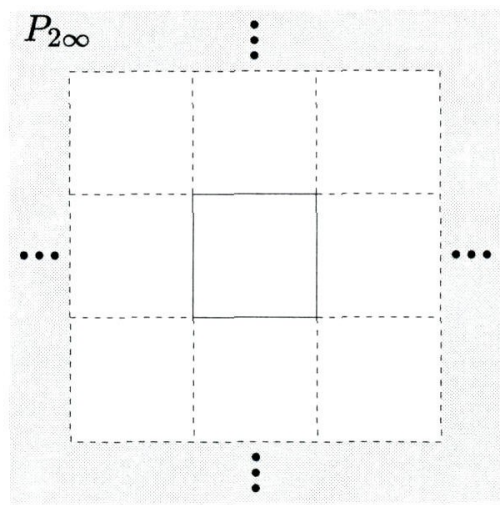

(d)

Figure 6.1: Images about the origin of a doubly periodic function. Shaded regions are labelled: the (a) base period $P_{0}$, (b) first ring $P_{1}$, (c) first and all remaining rings $P_{1 \infty}$, (d) second and all remaining rings $P_{2 \infty}$. Black circles represent the accompanying lattice of period centered points, $\tilde{P}_{i}$. In (c) and (d) the shaded region and lattice extend without bound.

where

$$
b_{n}=\sum_{m=1}^{p} a_{m}\left(\begin{array}{c}
n+m-1 \\
m-1
\end{array}\right)(-1)^{m} \frac{1}{w^{n+m}} .
$$

The series is truncated after $p$ terms to match the order of the multi-pole expansion. We now define a new series $\psi(z)$ which includes the influence of all periodic images 
$P_{2 \infty}$ on the target point $z$ as

$$
\psi(z)=\sum_{w \in \tilde{P}_{2 \infty}} \sum_{n=0}^{p} b_{n} z^{n}
$$

where $\tilde{P}_{2 \infty}$ represents the lattice of points in the center of each periodic image in $P_{2 \infty}$ (again, see Fig. 6.1). Finally, the order of summations in Eq. 6.5 is reversed, giving

$$
\psi(z)=\sum_{n=0}^{p} c_{n} z^{n}
$$

where the new coefficients are

$$
\begin{aligned}
c_{n} & =\sum_{m=1}^{p} a_{m}\left(\begin{array}{c}
n+m-1 \\
m-1
\end{array}\right)(-1)^{m} \sum_{w \in \tilde{P}_{1 \infty}} \frac{1}{w^{n+m}} \\
& =\sum_{m=1}^{p} a_{m}\left(\begin{array}{c}
n+m-1 \\
m-1
\end{array}\right)(-1)^{m} S_{n+m},
\end{aligned}
$$

and $S_{n+m}$ need only be calculated once, for each of the various powers $(m+n)$. In this example, $S_{1}$ through $S_{2 p}$ may be required. An efficient way to calculate these sums numerically is presented in [7], but since this is only a one-time calculation, any brute force method is sufficient. Thus we have an expression, $\psi(z)$, which designates the "influence" of all distant periods $P_{2 \infty}$. The only information we need provide is the multi-pole coefficients $a_{m}$ and the one-time sums $S_{i}$.

\subsection{Implementation}

The technique in the previous section carries over into the realm of evaluating the influence of all distant periodic images $\left(P_{2 \infty}\right)$ of the doubly periodic vortex sheet (SWP2). Consider a shear layer vortex sheet in the $x-y$ plane. The base period $P_{0}$ represents the base periodic form of the vortex sheet. We recognize that the previous multi-pole expansion of the Biot-Savart kernel (Eq. 5.7) is applicable here, simply by 
considering $V$ to be the volume of a larger box surrounding one period of the entire sheet ( $P_{0}$ for example) instead of the smaller boxes used in Chapter 5 . Comparing with the example in the previous section, Eq. 6.2 is analogous to Eq. 5.7, $z$ is analogous to $\boldsymbol{x}$ and $w$ is analogous to $\tilde{\boldsymbol{x}}$. As in the example, the multi-pole expansion about $\tilde{\boldsymbol{x}}$ is converted (with sufficient differentiation) to a Taylor series about the origin and we have (in index notation)

$$
u_{i}^{V}(\boldsymbol{x})=B_{i}^{0}+B_{i j}^{1} x_{j}+B_{i j k}^{2} x_{j} x_{k}+\cdots
$$

where the coefficients $B^{n}$ are functions of the moments $M^{n}$ (of any one period, say $P_{0}$ ) and $\tilde{\boldsymbol{x}}$ (the center or lattice point of the periodic image under consideration, $V$ ). Finally this Taylor series is summed for each periodic image in $P_{2 \infty}$ (i.e., $\tilde{x} \in \tilde{P}_{2 \infty}$ ) resulting in an expression for the influence of all periodic images in $P_{2 \infty}$ on the field point $\boldsymbol{x}$ near the origin:

$$
u_{i}^{P_{2 \infty}}(\boldsymbol{x})=C_{i}^{0}+C_{i j}^{1} x_{j}+C_{i j k}^{2} x_{j} x_{k}+\cdots
$$

where the $C^{n}$ are functions of the moments $M^{n}$ and the double periodic sums $S_{i, j}$, analogous to the sums $S_{i}$ in Eq. 6.7. Again the summations $S_{i, j}$ need only be calculated once and simply stored. Only 12 are needed for a second order expansion, as depicted in Tab. 6.1. $S_{1,1}$, for example, is

$$
S_{1,1}=\sum_{\left(x_{i}, y_{i}\right) \in \tilde{P}_{2 \infty}} T_{i}^{1,1}=\sum_{\left(x_{i}, y_{i}\right) \in \tilde{P}_{2 \infty}} \frac{x_{i}^{2}}{D_{\sigma, i}^{5}}
$$

where $D_{\sigma, i}=\left[x_{i}^{2}+y_{i}^{2}+\sigma^{2}\right]^{1 / 2}$. See Appendix E for details on evaluating these doubly infinite sums.

Thus the total velocity at a target point $\boldsymbol{x}$ is calculated by using the standard box-target interactions in $P_{0} \cup P_{1}$, but the influence of the entire $P_{2 \infty}$ is given by one simple Taylor series. The accuracy of this technique simply depends on the number of terms retained in the Taylor expansion (Eq. 6.8 and Eq. 6.9), assuming the moments 


\begin{tabular}{c|cccc} 
& 1 & $x_{m}^{2}$ & $x_{m}^{2} y_{m}^{2}$ & $x_{m}^{4}$ \\
\hline$D_{\sigma, m}^{3}$ & $T_{m}^{0,0}$ & - & - & - \\
$D_{\sigma, m}^{5}$ & $T_{m}^{0,1}$ & $T_{m}^{1,1}$ & - & - \\
$D_{\sigma, m}^{7}$ & $T_{m}^{0,2}$ & $T_{m}^{1,2}$ & - & - \\
$D_{\sigma, m}^{9}$ & $T_{m}^{0,3}$ & $T_{m}^{1,3}$ & $T_{m}^{2,3}$ & $T_{m}^{3,3}$ \\
$D_{\sigma, m}^{11}$ & - & $T_{m}^{1,4}$ & $T_{m}^{2,4}$ & $T_{m}^{3,4}$
\end{tabular}

Table 6.1: Terms $T^{i, j}$ of the double summations $S_{i, j}$ needed to evaluate $C^{n}$. The first row represents the numerator and the first column represents the denominator of the summed term in each $S_{i, j}$, as exemplified in Eq. 6.10.

$\left(M^{n}\right)$ and sums $\left(S_{i, j}\right)$ are calculated accurately.

For the implementation utilized in this thesis the third order (and higher) moments are not calculated (as in \$5.4) and the Taylor series above is similarly truncated after the second order terms. Thus, in order to achieve sufficient accuracy, Eq. 6.8 and Eq. 6.9 are written slightly generalized as series about an arbitrary point $\boldsymbol{x}_{a}$ instead of restricting the series to be centered at the origin. Five points are chosen in the base period $\left(P_{0}\right)$ around which these Taylor series are expanded such that the expansion radius in a velocity evaluation, $\left|\boldsymbol{x}-\boldsymbol{x}_{a}\right|$ and hence the expansion error, is sufficiently small for all $\boldsymbol{x}$ in $P_{0}$. 


\section{Chapter 7 Performance}

In this chapter we examine the collection of run-time parameters which need to be set prior to each calculation and their influence over the final solution quality. Suitable choices are made for many of the parameters which are valid for all subsequent calculations. In addition we attempt to show the correctness of the simulation code by comparing it with common 2D calculations and exact analytic solutions. Convergence with respect to several parameters is discussed. Finally we show the overall speed improvement we have achieved over the direct $O\left(N^{2}\right)$ method. Most of the studies presented in this chapter are based on an example initial condition, described below.

\subsection{Example Initial Condition}

The example regularized vortex sheet configuration is seemingly odd in that it would likely be quite difficult to create experimentally. However, it provides an excellent testing ground for two basic vortex sheet phenomena in one simulation: standard roll-up and also a nearly self-impinging surface. In general, the initial condition creates a flow similar to a closed, circular jet (with circular cross-section) inside a toroidal surface. Vortex lines are closed circles around the tube of the toroidal surface. The strength of each vortex line is varied in this case, such that the jet impinges on itself, as depicted in Fig. 7.1.

Specifically, the initial condition is a SWP0 surface as follows:

$$
\mathbf{X}=\left(\begin{array}{c}
0.25 \sin \eta \\
(1+0.25 \cos \eta) \cos \xi \\
(1+0.25 \cos \eta) \sin \xi
\end{array}\right) \quad \Gamma=\cos \xi
$$




\subsection{Run-time Parameters}

Several parameters must be determined for each simulation to run at peak effciency in terms of CPU time versus error in the computation. In order to determine these parameters, several simulations of the example vortex sheet (from \$7.1) are computed with different values for the parameters. All runs are variants of the following parameter set: $\sigma=0.2$ (relative to initial tube diameter), $R=40, E_{T O L}=0.0001$, 6 point Gaussian quadrature, $\Delta t=0.05$, automatic mesh refinement turned off. Henceforth all time integration is performed with RK2. To compare different runs, we examine a selection of four of the invariants: volume, helicity, and hydrodynamic and angular impulse magnitude. The volume is scaled by the exact initial volume, $V_{0}=\pi^{2} / 8$, as is the hydrodynamic impulse, with $\left|\mathbf{I}_{0}\right|=\pi^{2} / 16$. Preservation of the invariants is a necessary but not sufficient condition for a successful, accurate simulation. Of course without remeshing, each case is destined for failure after some time; thus the following plots are examined to characterize the onset and rate of departure from constancy. In Fig. 7.2 the resolution is varied with the other parameters held constant. In Fig. 7.3 the box-target error tolerance $E_{T O L}$ is varied with the other parameters held constant. In Fig. 7.4 the number of Gauss integration points is varied with the other parameters held constant. In Fig. 7.5 the time step $\Delta t$ is varied with the other parameters held constant. It is interesting to note that the invariants are insensitive to some parameters, but highly sensitive to others. Assuming the influence of the parameters is somewhat independent, we choose $R=20, E_{T O L}=0.001$, number of Gauss points $=6, \Delta t=0.05$ as representing a solution with sufficient accuracy. This case is shown in Fig. 7.6, with various remesh parameters. Note that in all of these cases, the time integration is stable.

\subsection{Remeshing Frequency}

Having decided on typical values to use for the run-time parameters, we now examine the benefits and drawbacks of remeshing at various frequencies, based on the 
thresholds $t_{N C}$ and $t_{L R}$. As depicted in Fig. 7.6, the less frequent mesh refinement trigger seems to be the best trade-off between small scale adaption and invariant stability. The three sheet surfaces consist of, at the final time, 3200, 16500, and 24500 triangles, following the red, green and blue data respectively.

\subsection{Convergence Analysis}

There are several convergence properties to check. Unfortunately spatial convergence is somewhat difficult to characterize due to the adaptive remeshing involved. Thus we examine three spatial convergence issues independently: (1) the spatial interpolants, (2) overall spatial convergence with a regular grid, and (3) qualitative convergence inspection with the adaptive remeshing.

To examine convergence of the interpolation scheme, we again use the example initial condition (from $\$ 7.1$ ). Fig. 7.7 shows the convergence properties of the interpolation procedure, combining data for all nine functions in $\mathbf{X}, \mathbf{X}_{\xi}$, and $\mathbf{X}_{\eta}$. In this case, "error" $E$ is defined as the maximum difference between the exact and interpolated values, as determined by a 10000 point sample over a regular grid spanning the parameter space:

$$
E=\max \left(\mathbf{X}_{i, \text { exact }}-\mathbf{X}_{i \text {,interp }}, i=1 \text { to } 10000\right)
$$

Assuming $E$ is proportional to $N^{m}$ and also to $\Delta X^{-2 m}$ we see that $\mathbf{X}$ is third order accurate in $\Delta X$ and the derivatives are second order accurate. The triangular Gaussian quadrature integration was confirmed to behave as expected, integrating low order polynomials exactly as designed.

The overall order of accuracy in space is also determined from the example initial condition. For this calculation, the sheet is not triangulated with the advancing front procedure; instead a "regular" triangular grid is generated, with vertices located at evenly spaced intervals in $\Sigma$. With this discretization, it is possible to characterize the spatial order of convergence with successively generated grids, halving the average 
length scale with each successive iteration. We generate data from two sets of runs, one set with the number of Gauss points at 4 , the other at 6 . In each set, 3 simulations are run for 10 time steps with $\Delta t=10^{-5}, E_{T O L}=0$, no remeshing and $R=5,20,80$. Assuming vertex locations then behave as

$$
\left|\mathbf{X}_{\text {exact }}-\mathbf{X}_{\text {calc }}\right|=A|\Delta \mathbf{X}|^{n}
$$

(i.e., the error is dominated by factors relating to spatial resolution) where $A$ is a constant, and the average length scale decreases by a factor of 2 with each increase in $R$, we have for $n$, the spatial order of convergence,

$$
n=\frac{1}{\log 2} \log \frac{\left|\mathbf{X}_{c 2}-\mathbf{X}_{c 1}\right|}{\left|\mathbf{X}_{c 3}-\mathbf{X}_{c 2}\right|}
$$

where subscripts $c i$ represent the $i^{\text {th }}$ calculation as the resolution varies from $R_{1}=5$ to $R_{3}=80$. Fig. 7.8 shows the point-wise spatial order of convergence for both 4 and 6 point Gaussian quadrature. In both cases, $n$ varies spatially from about 1.5 to 4 , the average value increasing from 2 (with the 4 point integration) to 2.5 with the 6 point integration.

To further characterize the spatial convergence, we examine three cases with $R$ $=10,20$ and 40; all other parameters are as decided in $\S 7.2$ and $\S 7.3$. A single slice through the rings (as a mesh skeleton) is shown in Fig. 7.9. The three cases do converge to the same solution. The low resolution case $(R=10)$ is distinguished from the other two, but the difference between $R=20$ and $R=40$ is difficult to assess.

Lastly we recall that in $2 \mathrm{D}$ the limit as $\sigma \rightarrow 0$, past the singularity time appears to exist, at least in the outer loops of the roll-up region [27]. We have no reason to believe this characteristic does not hold in $3 \mathrm{D}$ as well. In Fig. 7.10 below, the example condition is presented at $t=1.8$ with the kernel smoothing parameter set at $\sigma=0.1$ (one half of its previous value) relative to the initial diameter. Clearly the roll-up begins at earlier time and the outer shape is qualitatively similar. Following this trend, one can begin to imagine the limiting case for $\sigma=0$. 


\subsection{Comparison with Exact Velocities}

\subsubsection{SWP1}

Consider a cylindrical vortex sheet (SWP1) with unit radius and strength, aligned along the $x$ axis. It may be parameterized as

$$
\mathbf{X}=\left(\begin{array}{c}
\xi \\
\sin (\eta) \\
\cos (\eta)
\end{array}\right), \quad \Gamma=\xi
$$

with $(\xi, \eta) \in(-\infty . . \infty,-\pi . . \pi)$. Substitution into Eq. 2.22 yields the velocity as a function of $r$, the radial distance from the $x$ axis. Specifically

$$
U=\frac{1}{2 r \sqrt{\alpha}}\left(1+\sigma^{2}-r^{2}-\sqrt{\alpha}\right)
$$

where

$$
\alpha=\left[(1-r)^{2}+\sigma^{2}\right]\left[(1+r)^{2}+\sigma^{2}\right] .
$$

Fig. 7.11 shows how the numerical method (points) compares to the above analytic formulas, for decreasing values of $\sigma$. As $\sigma$ decreases, the velocity profile from the regularized vortex sheet approaches that of the true vortex sheet. In this case, the velocity of the vortex sheet is $U=1$; thus we confirm the velocity of the regularized sheet approaches that of the vortex sheet as $\sigma$ decreases. 


\subsubsection{SWP2}

We parameterize a planar vortex sheet (SWP2) modeling a shear layer, as depicted in Fig. 11.1, via

$$
\mathbf{X}=\left(\begin{array}{c}
\xi / 2 \pi \\
\eta / 2 \pi \\
0
\end{array}\right), \quad \Gamma=-\xi / 2 \pi
$$

The exact velocity profile above $(z>0)$ this vortex sheet from the regularized kernel (Eq. 2.22) is

$$
U=\frac{1}{2} \frac{-z / \sigma}{\sqrt{1+(z / \sigma)^{2}}}
$$

Fig. 7.12 shows a sampling of calculated velocities, for two different values of $\sigma$, compared to the exact analytic expression above. The agreement is good to a height of $5 \sigma$ above the sheet at which point the maximum absolute error in the velocity is 0.005 .

\subsection{Comparison with 2D Results}

\subsubsection{SWP1}

Several comparisons are possible with existing $2 \mathrm{D}$ results. Consider, for example, the case of a cylindrical vortex sheet, the strength of which is given by the slip velocity of an ideal fluid as a free stream passes around the cylinder. For comparison, the SWP1 surface type is used with initial condition

$$
\mathbf{X}=\left(\begin{array}{c}
\xi / 2 \pi \\
0.2 \sin (\eta) \\
0.2 \cos (\eta)
\end{array}\right), \quad \Gamma=-2 \sin (\eta)
$$


Each period in physical space has unit length and initial radius to period ratio of 0.2 . A separate $2 \mathrm{D}$ code, which compares perfectly with the results in $[50]$, is used as a test bed. The current $3 \mathrm{D}$ code is able to reproduce similar results, as shown in Fig. 7.13. The projected 3D solution in Fig. 7.13(a) is a snapshot in the time evolution with $\sigma=0.05$ (scaled with initial diameter), beginning with $\mathrm{N}=1000$ and ending with $\mathrm{N}=9400$ triangles. The projected $3 \mathrm{D}$ solution in Fig. 7.13 (b) is a snapshot in a different time evolution with $\sigma=0.025$ (scaled with initial diameter), beginning with $N=1000$ and ending with $N=27,000$ triangles.

\subsubsection{SWP2}

Another case for comparison is a shear layer with a purely $2 \mathrm{D}$ perturbation. Specifically, we consider

$$
\mathbf{X}=\left(\begin{array}{c}
\xi / 2 \pi+\epsilon \sin \xi \\
\eta / 2 \pi \\
-\epsilon \sin \xi
\end{array}\right), \quad \Gamma=-\xi / 2 \pi
$$

We have another purely $2 \mathrm{D}$ code which can duplicate the regularized calculations in [27], and is also used as a comparison test bed. Fig. 7.14 shows the $2 \mathrm{D}$ shear layer after a significant amount of time, with both the $2 \mathrm{D}$ curve and the projection of all $3 \mathrm{D}$ surface triangles onto a plane. Note the perfect agreement in the roll-up region but slight discrepancy at the edges of the period. Both calculations were performed with $\sigma=0.05$, relative to period length; $\epsilon=0.05$. The $3 \mathrm{D}$ calculation has a resolution of $R=20$, requiring 1000 triangles for the initial geometry, and 32000 at the final time, depicted. Further information about the purely 2D evolution equations, and their regularization, is in Appendix D. 


\subsection{Parallel Speedup}

As mentioned in $\S 3.3$ and $\S 3.7$, the simulation can be run on multiple processors in a shared memory environment. Fig. 7.15 shows the increase in execution speed relative to a single processor at each time step for an example case with 2,3 , and 4 processors. Clearly the speed-up is not ideal (four times faster with four processors), but this was to be expected as only the velocity evaluation routine is parallelized. Other sections of the program are run on a single processor only.

Fig. 7.16 shows the compound effect of the multi-pole velocity evaluation technique of Chapter 5 combined with parallel execution on four processors, relative to the direct $O\left(N^{2}\right)$ method on a single processor for the same example simulation. A 15-20 times speed improvement is significant. 


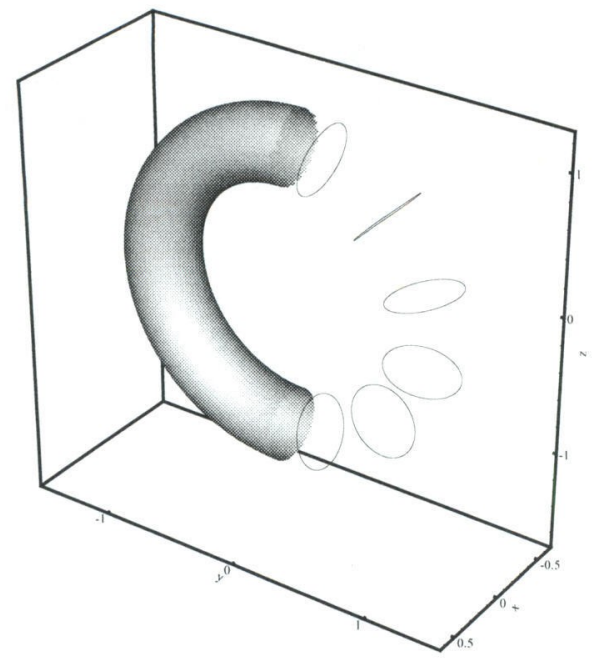

(a)

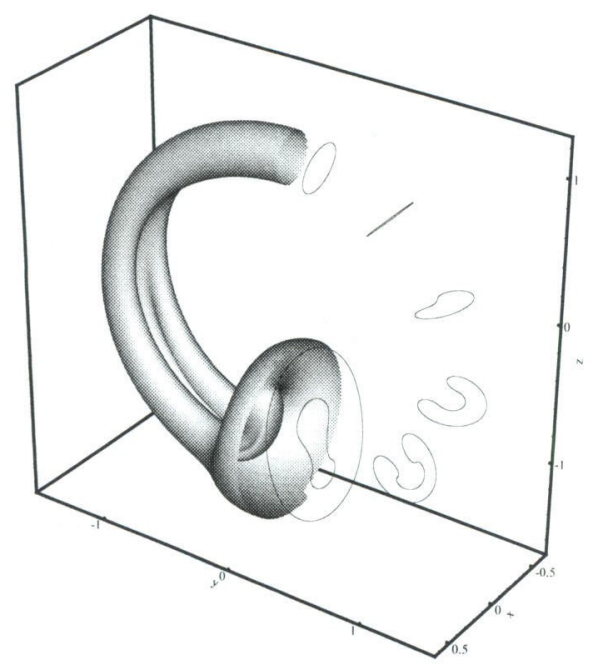

(c)

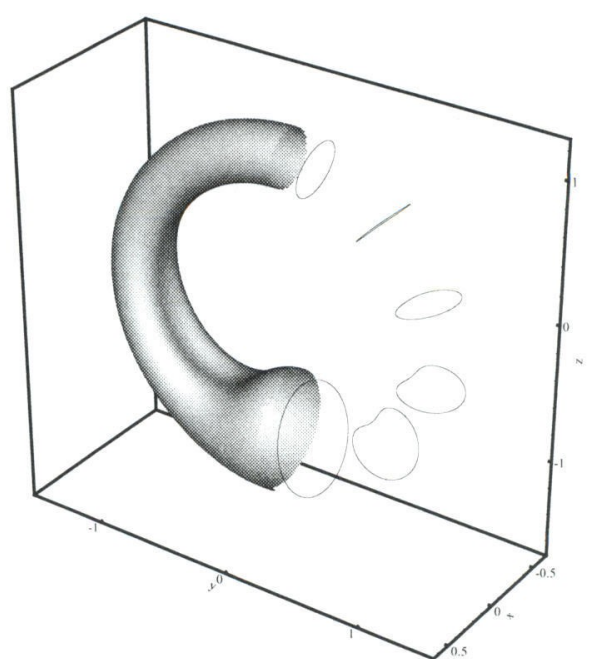

(b)

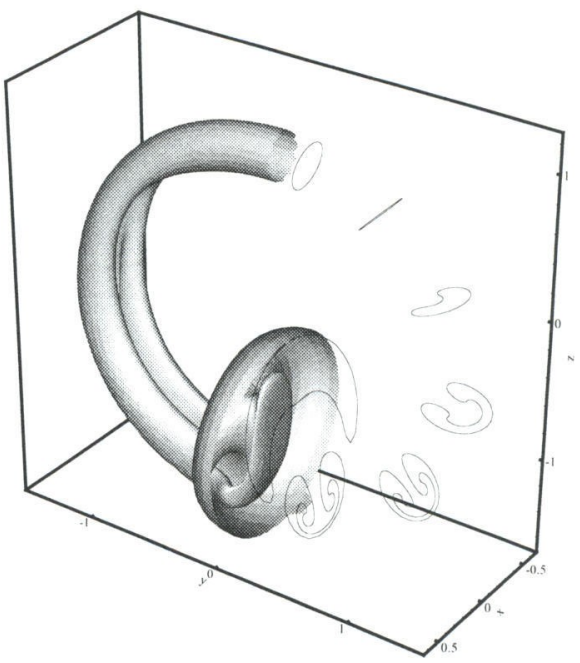

(d)

Figure 7.1: Shaded mesh skeleton of the example configuration, a self-impinging jet (SWP0), with $\sigma=0.2$ relative to initial tube diameter: (a) $t=0$, (b) $t=1.1$, (c) $t=2.2$, (d) $t=3.0$. Contours depict slices through the surface. 

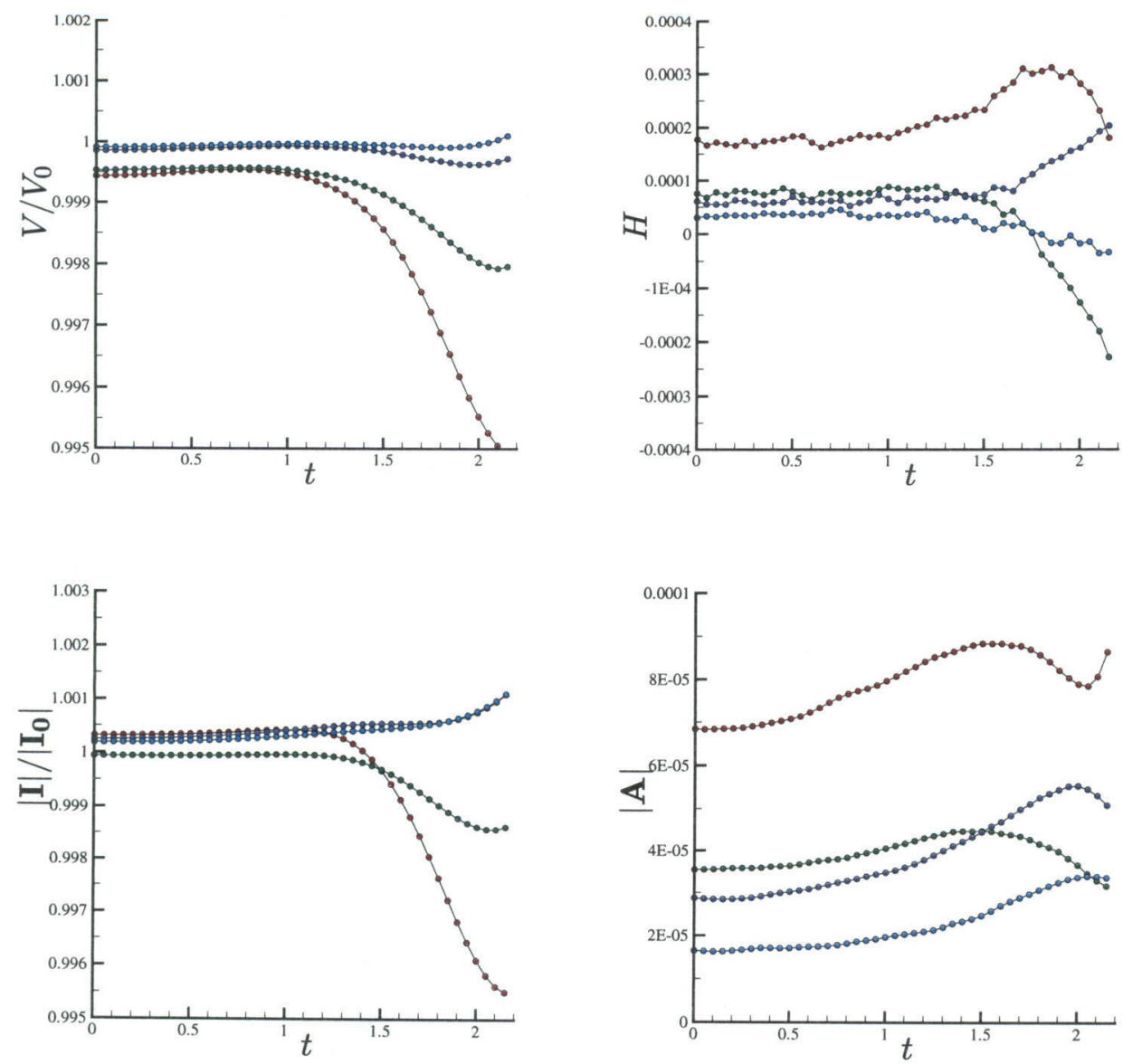

Figure 7.2: Variation of invariants with resolution $R . R=10$ (red), 20 (green), 30 (blue), 40 (cyan). Initial condition from $\S 7.1$. 

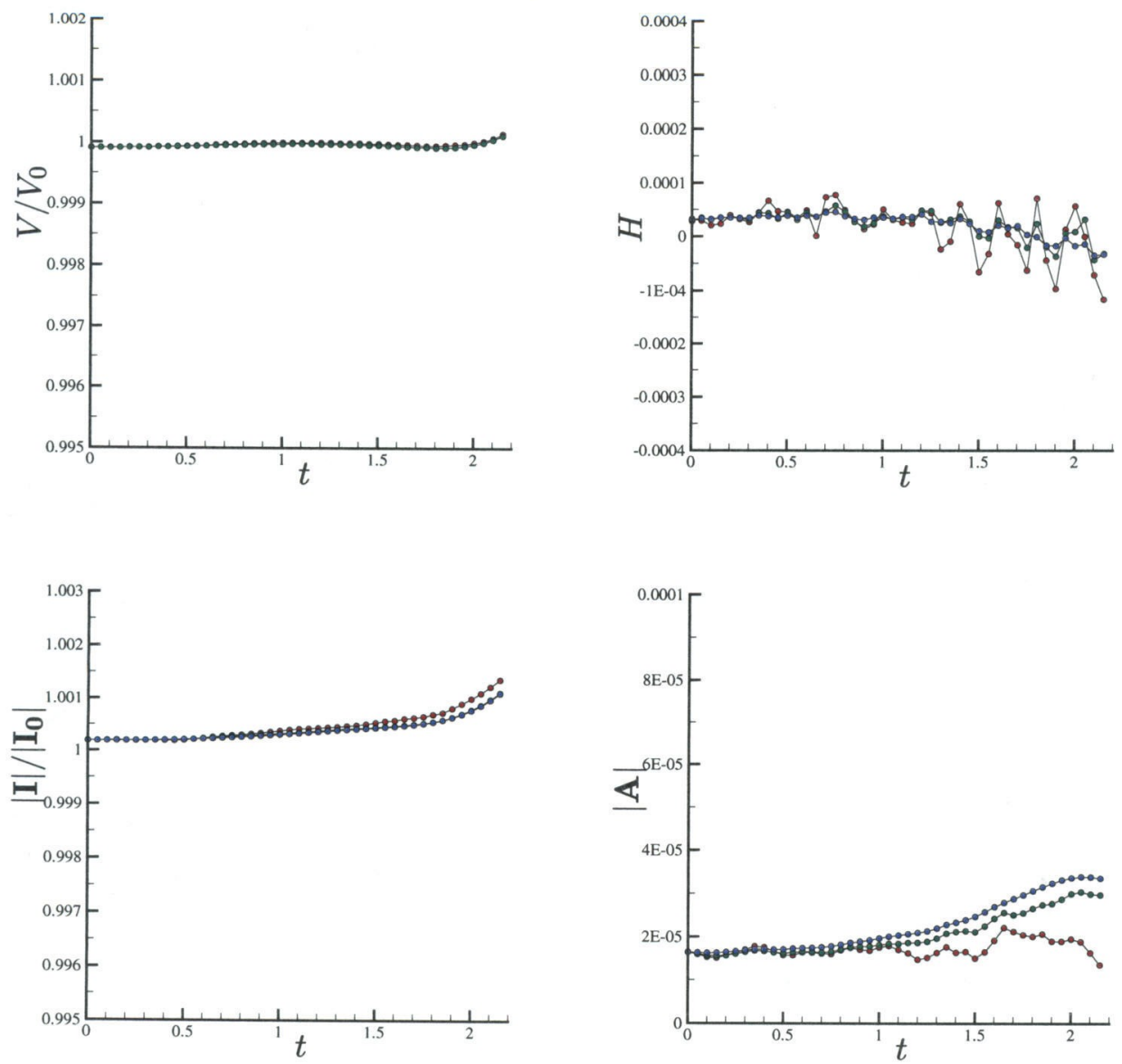

Figure 7.3: Variation of invariants with error tolerance $E_{T O L}: E_{T O L}=0.001$ (red), 0.0001 (green), 0 (blue). Initial condition from $§ 7.1$. 

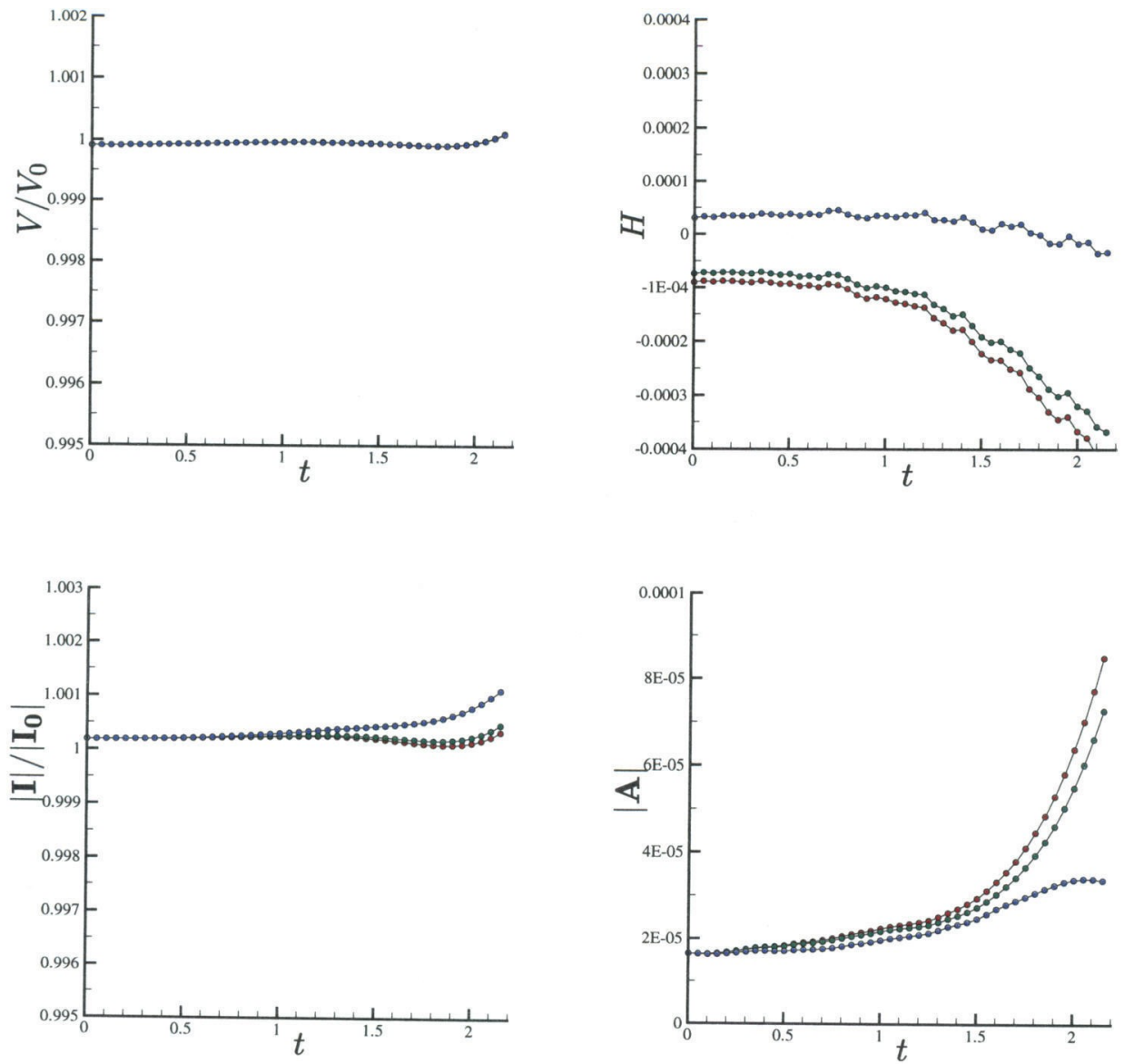

Figure 7.4: Variation of invariants with the number of Gauss integration points: 3 points (red), 4 (green), 6 (blue). Initial condition from $\S 7.1$. 

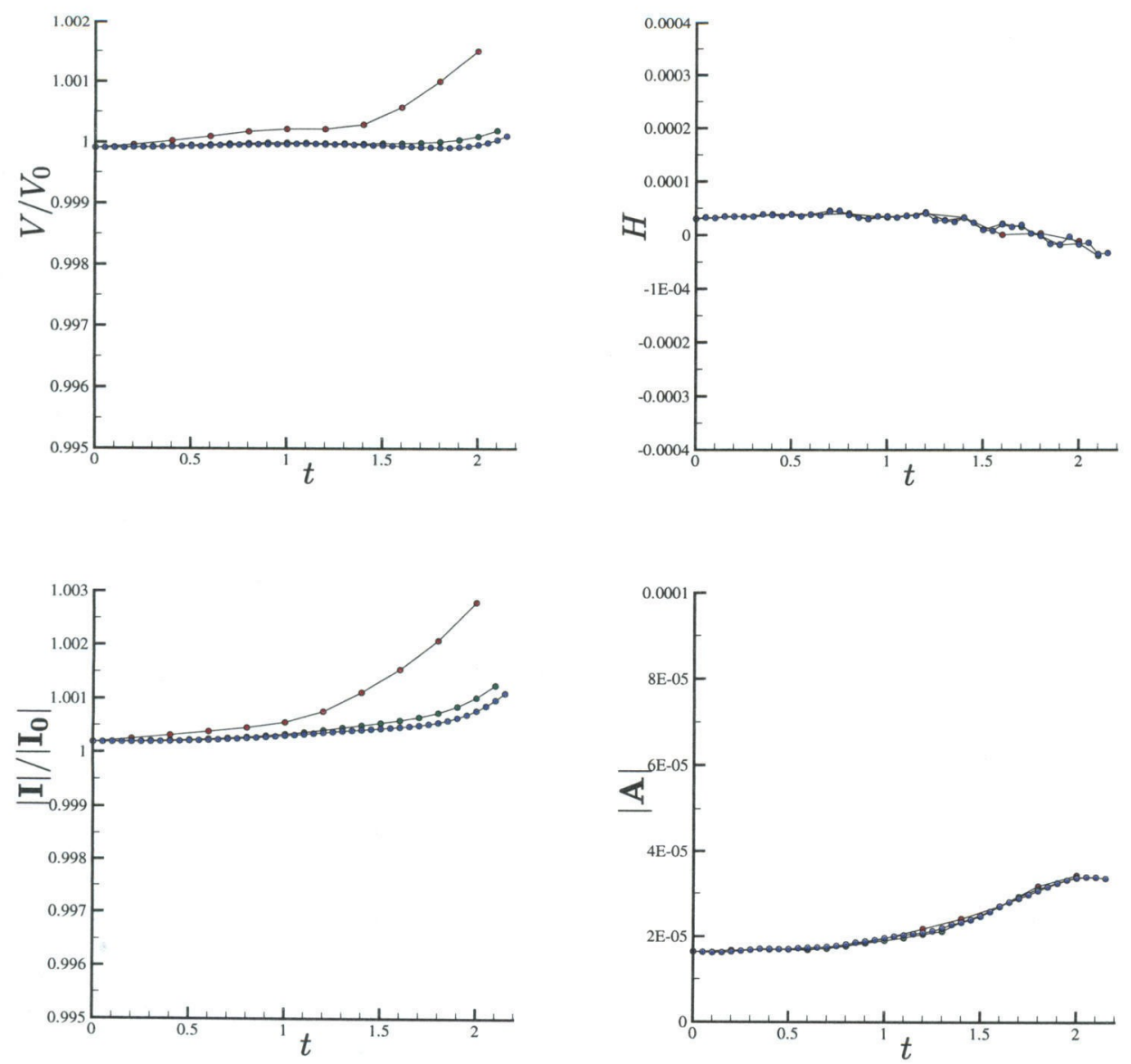

Figure 7.5: Variation of invariants with the time step $\Delta t: \Delta t=0.2$ (red), 0.1 (green), 0.05 (blue). Initial condition from $§ 7.1$. 

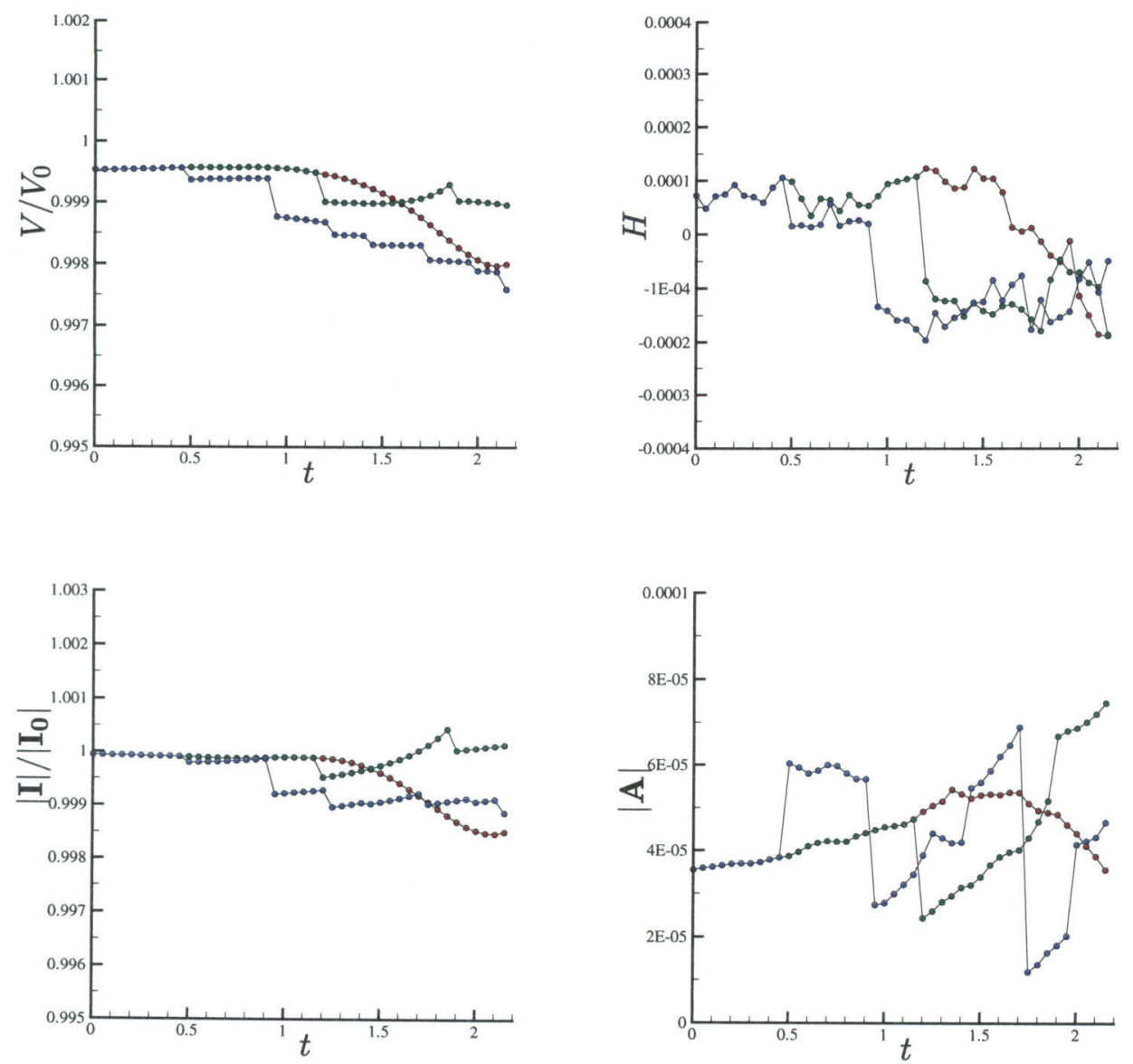

Figure 7.6: Variation of invariants with different mesh refinement triggering. Example simulation (from $\S 7.1$ ) with parameters chosen in $\S 7.2$. No remeshing (red), moderate remeshing with $t_{L R}=2, t_{N C}=20 \%$ (green), frequent remeshing with $t_{L R}=1.5$, $t_{N C}=10 \%$ (blue). 


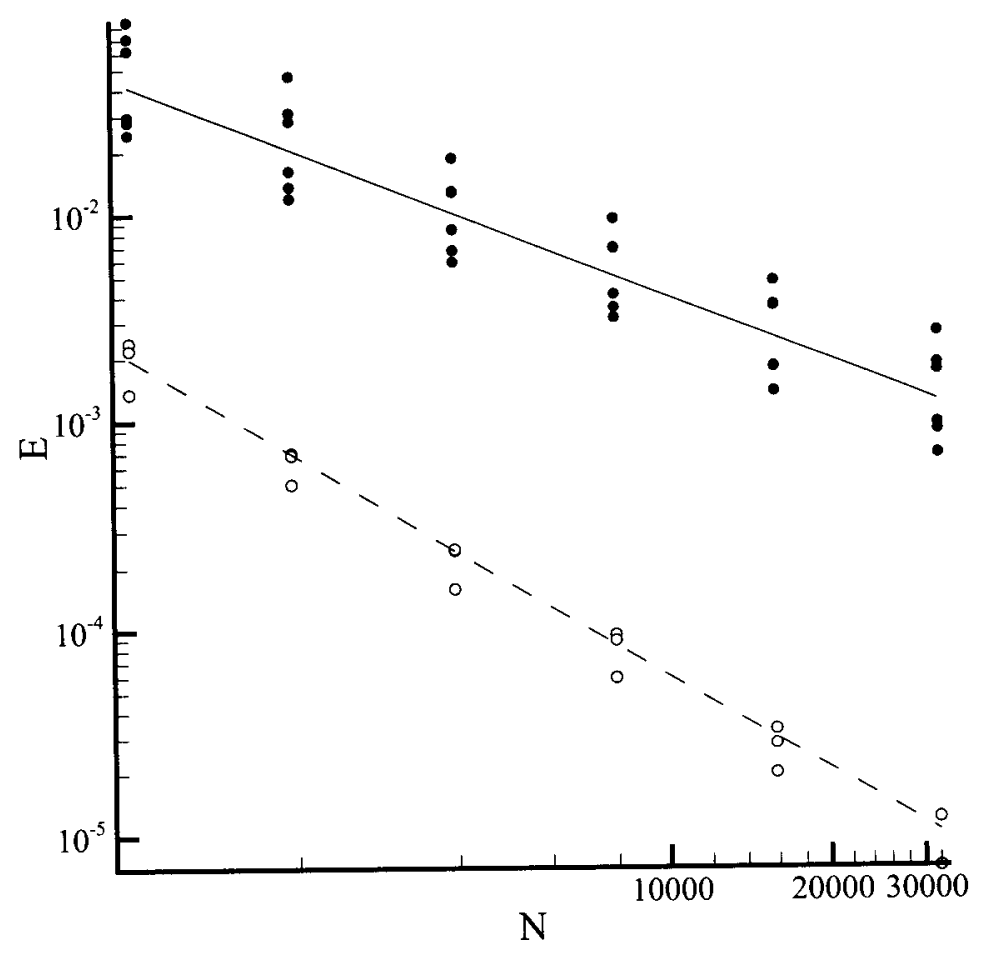

Figure 7.7: Maximum error $\mathrm{E}$ versus the number of triangles for each component of $\mathbf{X}$ (hollow points), $\mathbf{X}_{\xi}$ (filled), and $\mathbf{X}_{\eta}$ (filled). Dashed line has slope $m=-1.5$. Solid line has slope $m=-1.0$. 


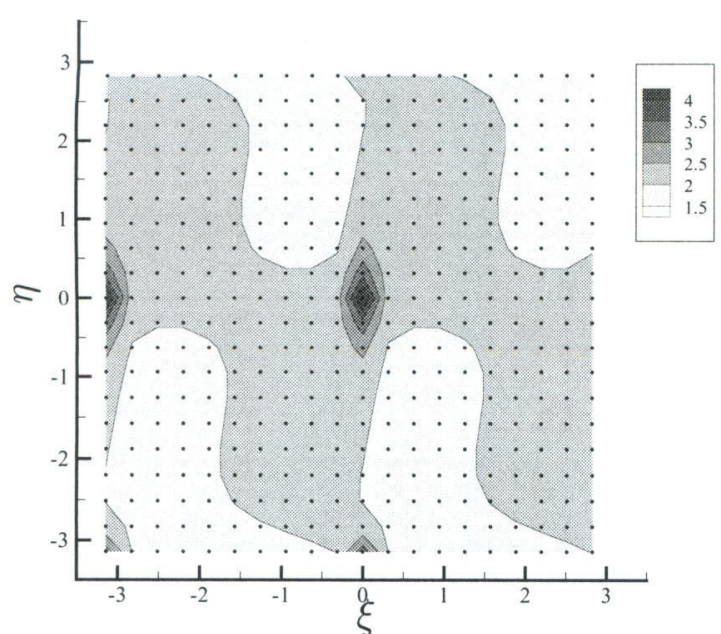

(a)

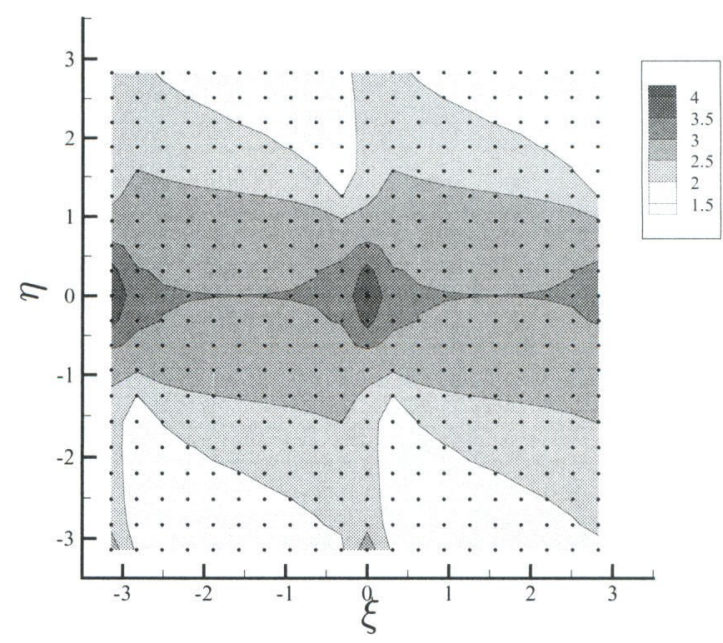

(b)

Figure 7.8: Spatial order of convergence with a regular grid and the example configuration as a shaded contour plot over the parameter space, $\Sigma$. Dots represent vertices for the lowest resolution case $(R=5)$. (a) 4 point Gaussian quadrature, (b) 6 point Gaussian quadrature.

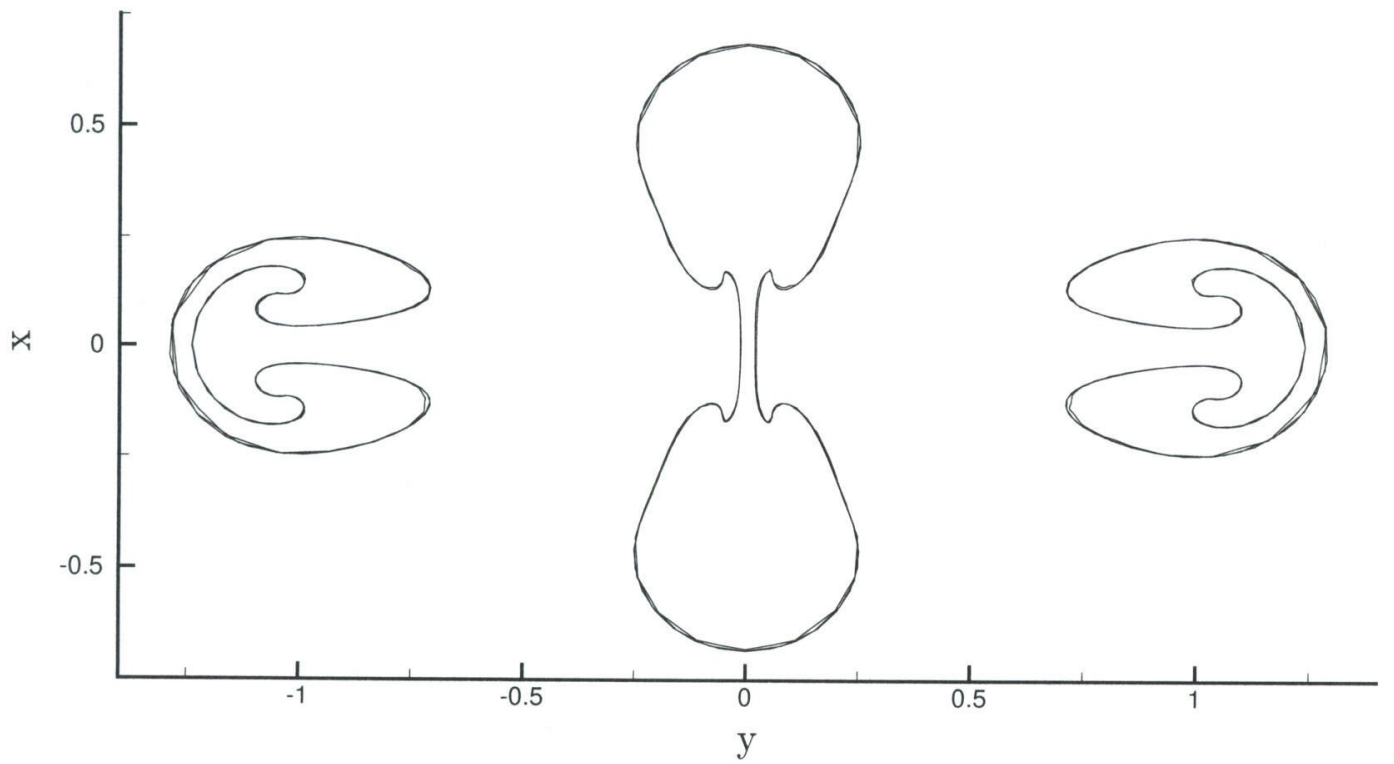

Figure 7.9: Qualitative spatial convergence demonstrated with a slice through the sheet surface at constant $z$ at $t=2.6, R=10,20,40$. 


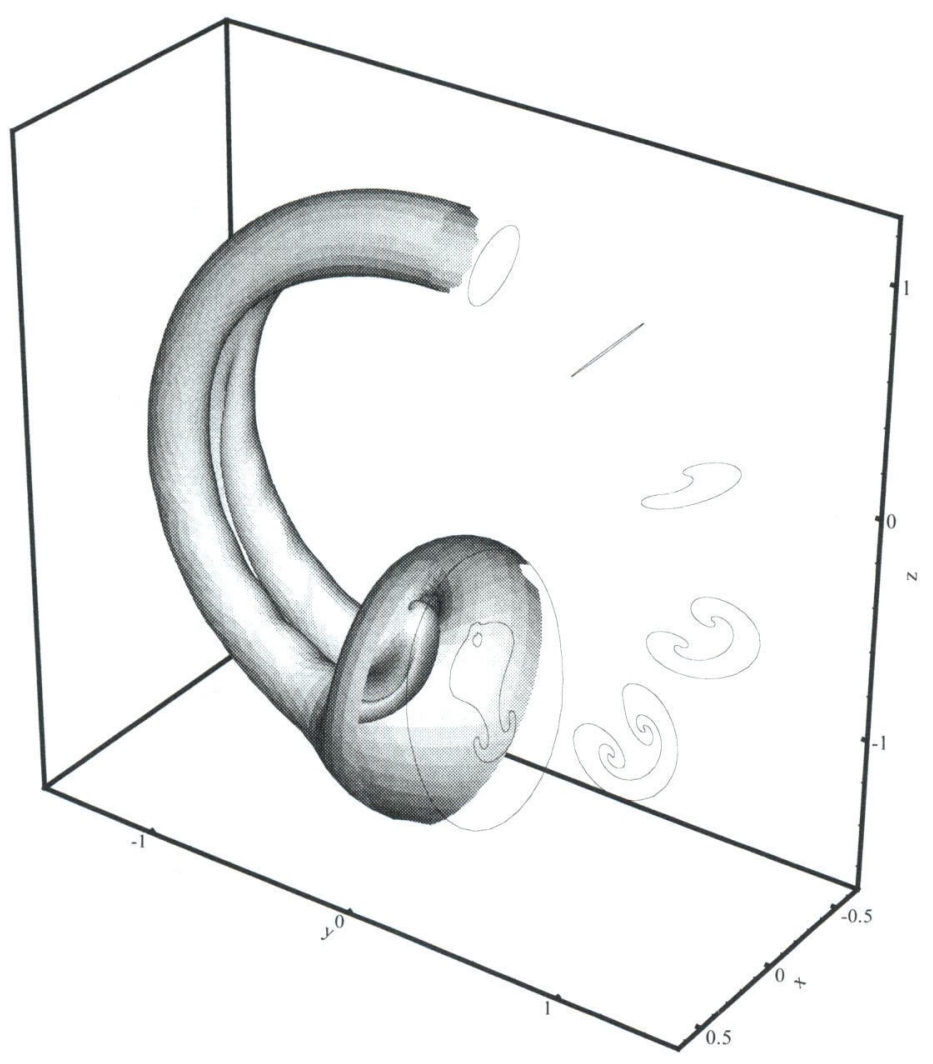

Figure 7.10: Shaded mesh skeleton and contour slices with reduced kernel smoothing parameter, $\sigma=0.1$ relative to initial diameter. Contours are slices through the surface. 


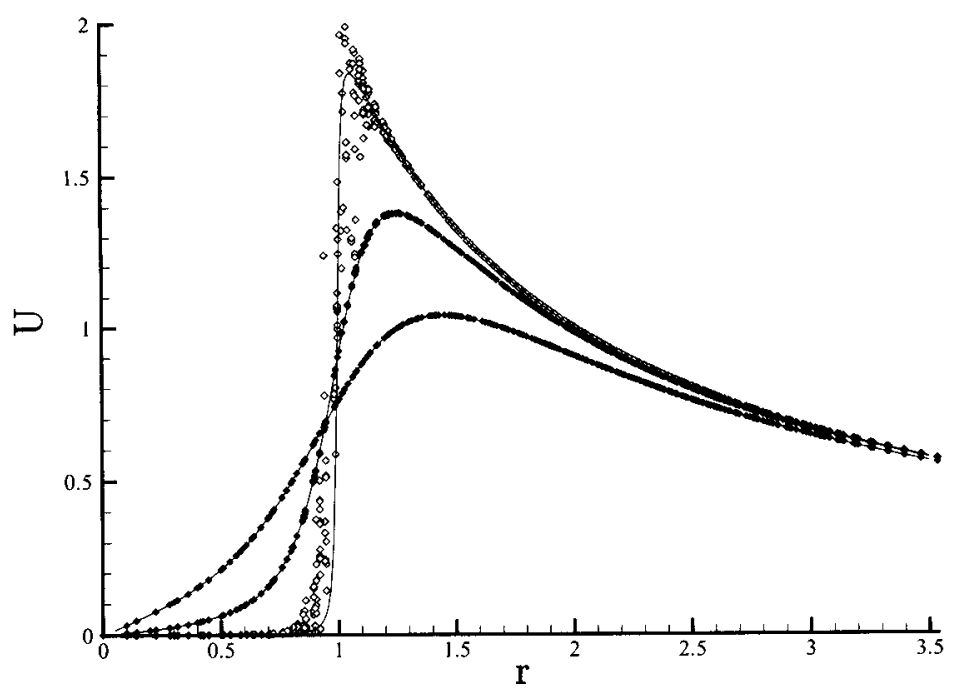

(a)

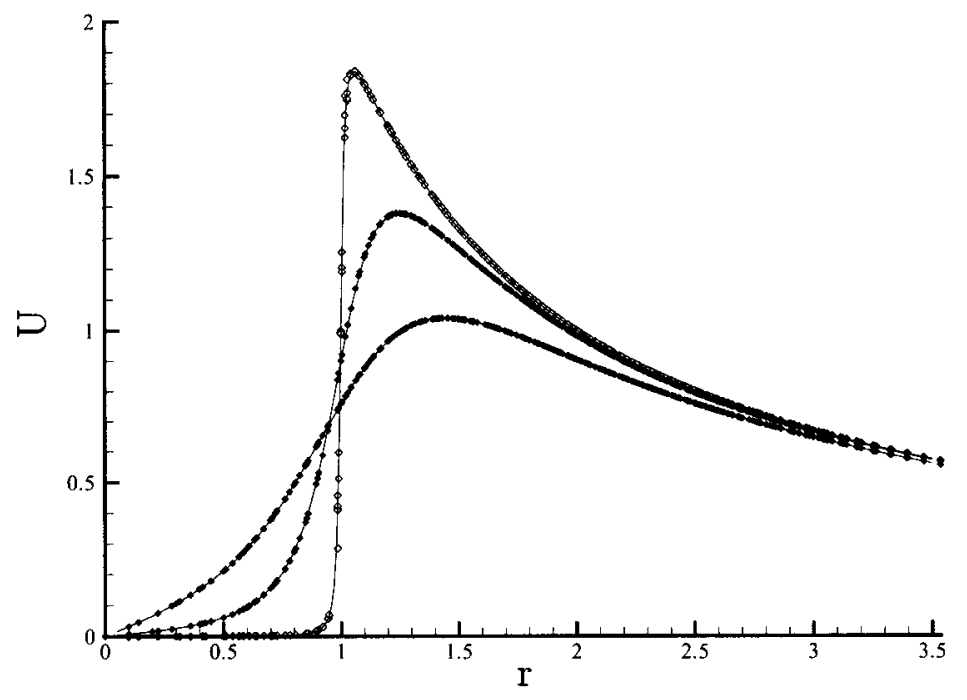

(b)

Figure 7.11: Comparison of numerical method (points) with exact velocities (lines) from the modified Biot-Savart kernel Eq. 2.22 with $\sigma=0.25,0.1$, (filled circles) and 0.01 (open circles). Curves steepen with decreasing $\sigma$. Note the dramatic difference in accuracy for $\sigma=0.01$ in (a) 500 and (b) 8000 triangles. 


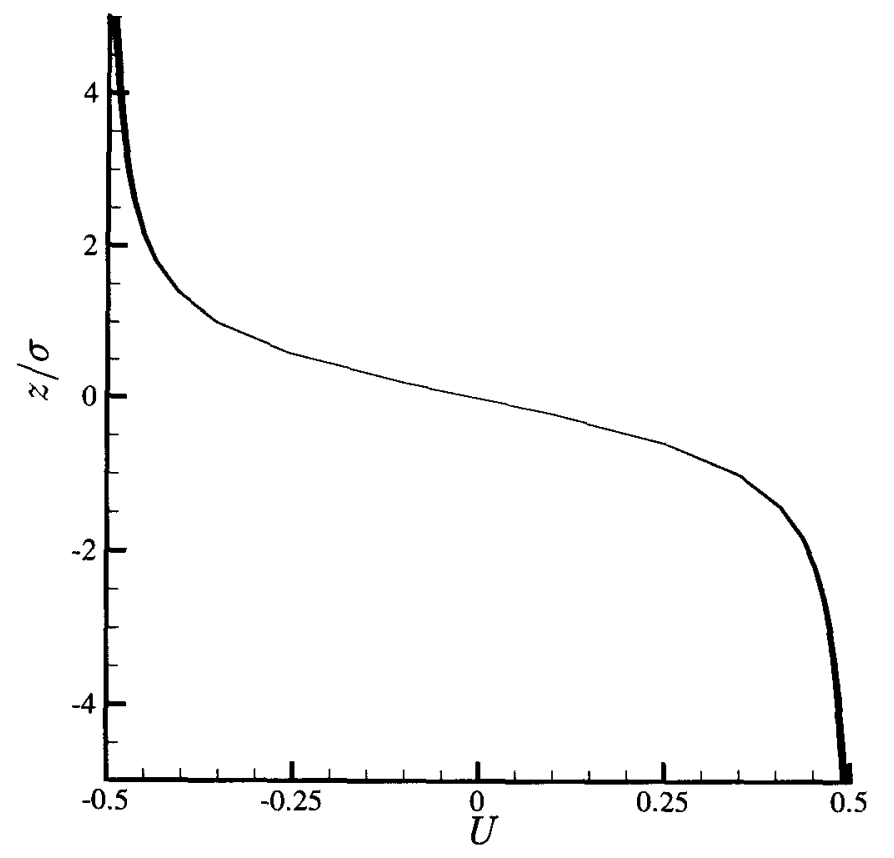

Figure 7.12: Sampled velocity profile above the regularized planar vortex sheet (red) and the exact solution from Eq. 7.9 (black). Samples taken from a uniform 25 by 25 grid over the sheet. Absolute error of 0.005 at a height of $5 \sigma$. 


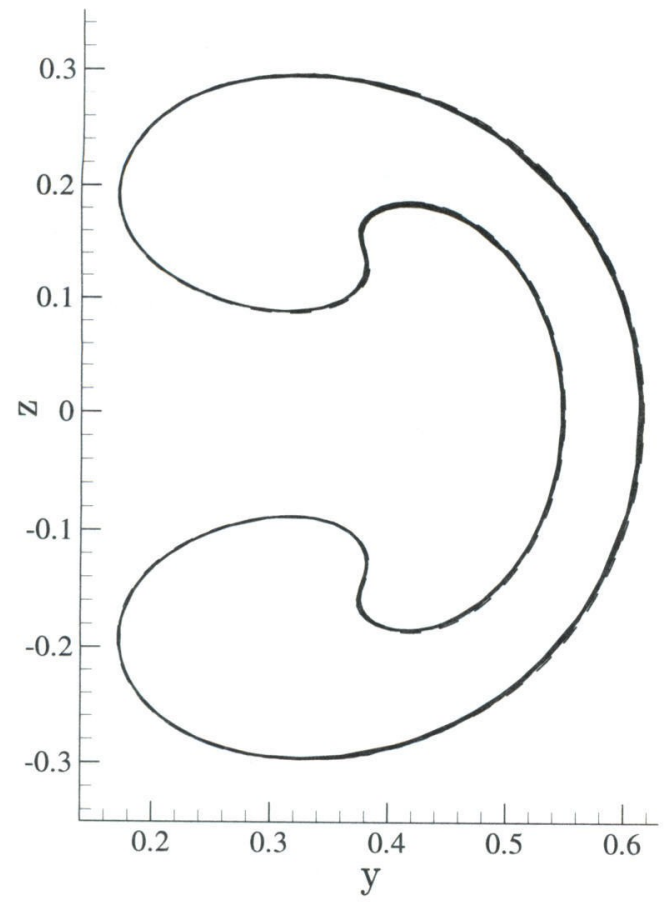

(a)

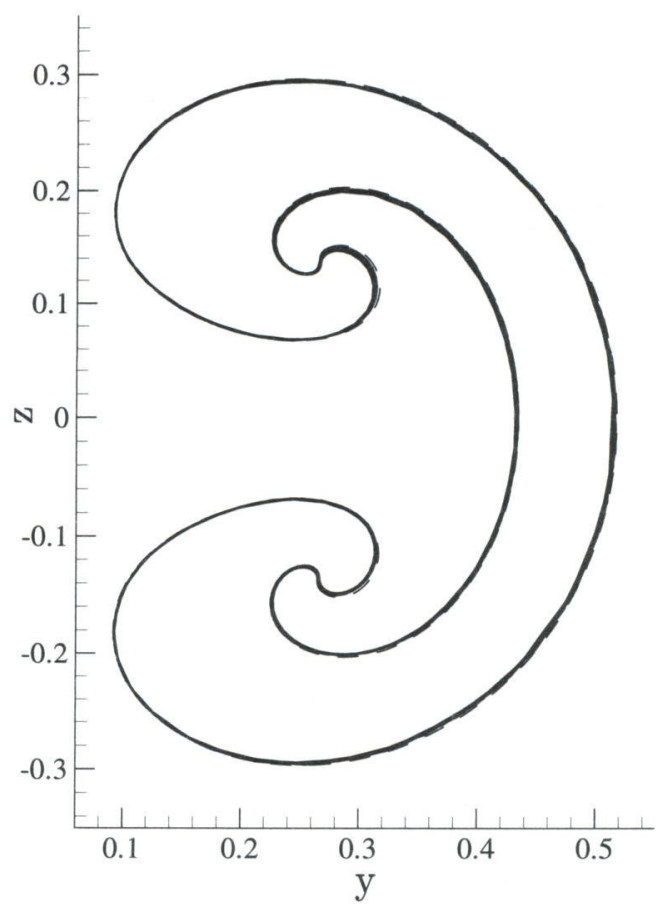

(b)

Figure 7.13: Comparison of current 3D method (solid lines representing surface, projected into $y-z$ plane) with 2D cylindrical vortex sheet (dashed lines). (a) $\sigma=0.1$ (b) $\sigma=0.05$.

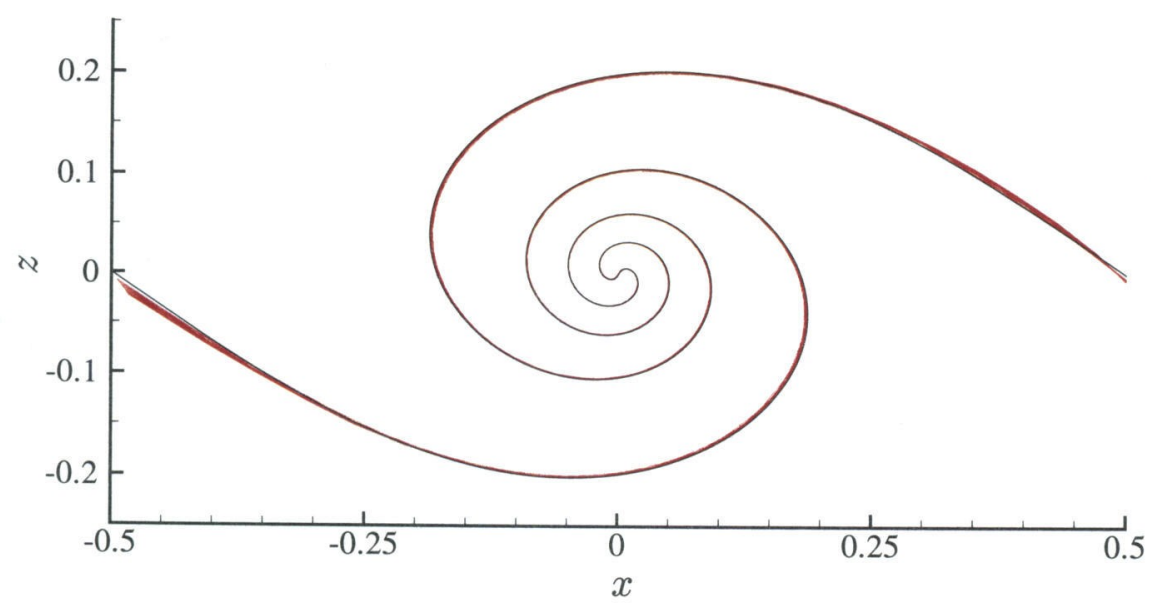

Figure 7.14: Comparison of current 3D method (SWP2 in red), projected into the the $(x-z)$ plane with 2D singly periodic vortex sheet (black line). $\sigma=0.05$, initial perturbation amplitude $\epsilon=0.05$. 


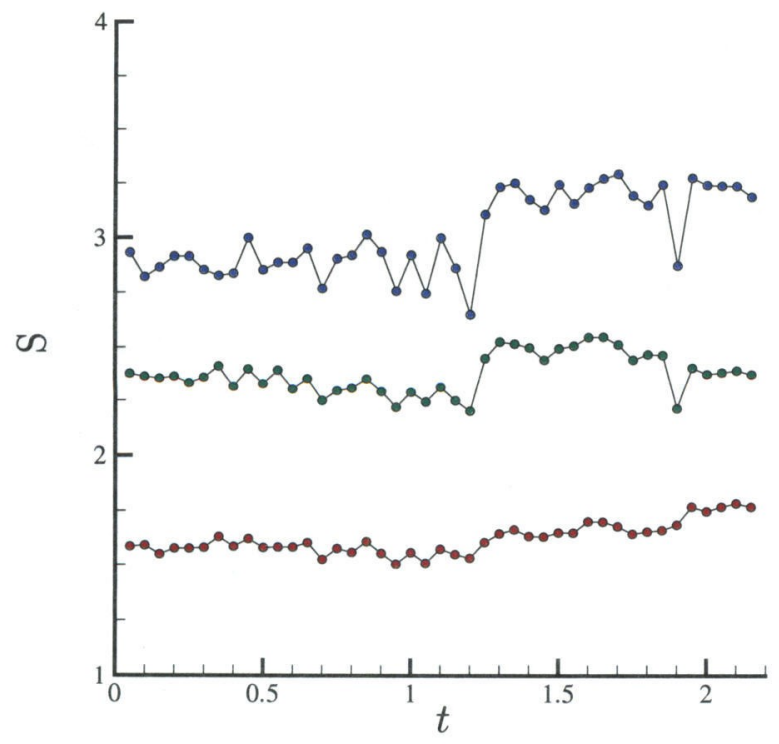

Figure 7.15: Program speed-up $S$ relative to a single processor execution at each time step of the example simulation (from §7.1). Two processors (red), three (green), and four (blue). Only the velocity evaluation routine is parallelized; hence the speed-up is not perfect.

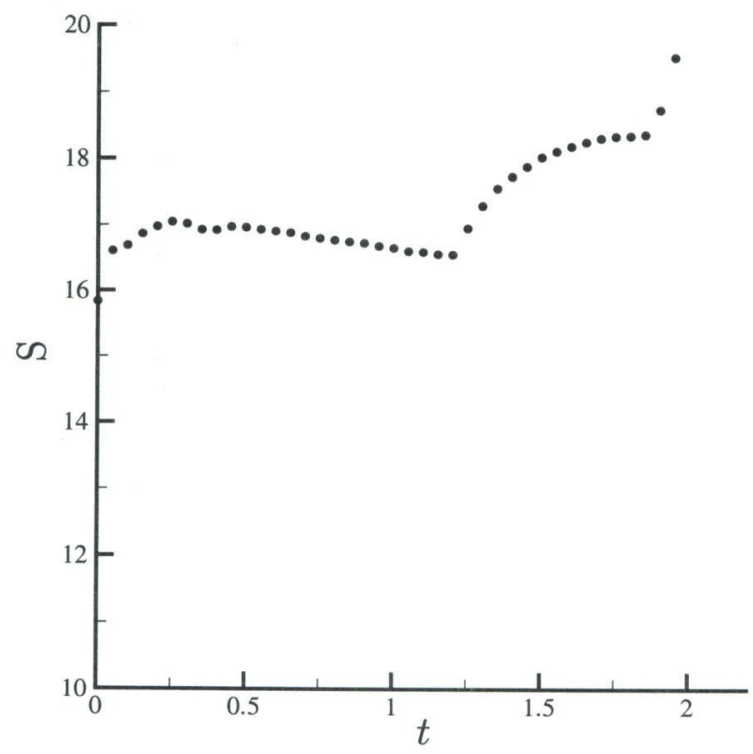

Figure 7.16: Cumulative program speed-up $S$ incorporating both four processors and the multi-pole velocity evaluation technique at each time step of the example simulation (from $\S 7.1$ ). 


\section{Chapter 8 Examples}

The following examples in this chapter show a sampling of different flows that can be modeled with the three surface types of regularized vortex sheets. In all cases, unless otherwise specified, the run time parameters are set as follows:

$$
\begin{aligned}
\text { time step: } & \Delta t=0.05 \\
\text { spatial resolution: } & R=20 \\
\text { remeshing thresholds: } & t_{L R}=2, t_{N C}=20 \% \\
\text { number of Gauss points: } & 6 \\
\text { box-target interaction error tolerance: } & E_{T O L}=0.001
\end{aligned}
$$

\subsection{Jet (SWP1)}

We first consider the growth of an axially perturbed jet with circular cross section. This is modeled with the SWP1 surface, and is thus constrained to periodic motion. We use the following initial conditions, defining the axial first mode geometric perturbation:

$$
\mathbf{X}=\left(\begin{array}{c}
\xi / 2 \pi \\
0.25[\sin (\eta)+0.1 \sin (\xi)] \\
0.25[\cos (\eta)+0.1 \sin (\xi)]
\end{array}\right) \quad \Gamma=-\xi
$$

This is a perturbation in the geometry only, not in the sheet strength and thus does not qualify as a true linear perturbation to the infinite, circular jet (see discussion in Chapter 9). An initial grid density of $R=20$ generates 500 triangles for the initial mesh, as shown in Fig. 8.1(a). Note that the time evolution preserves axisymmetry as seen by eye - an artifact of the relatively large $\sigma(=0.2$, relative to the initial average diameter). No symmetries are imposed by the computational method. By 
Fig. 8.1(d), we see significant roll-up of the sheet, essentially defining a vortex ring around the jet axis. At this time, the sheet is defined with 24,000 triangles per period, and computation becomes prohibitively expensive with the direct $O\left(N^{2}\right)$ method (Eq. 2.24). Fig. 8.1(e) shows the amount of detail and variety of scales present in the core of the vortex ring. Fig. 8.1(f) depicts the $C^{1}$ surface interpolant of the smooth sheet actually used in the computations. Fig. 8.2 shows the effect of varying $\sigma$ while keeping the initial surface type (duplicated from above) constant. Smaller values of $\sigma$ lead to faster roll-up as anticipated by Fig. 7.11. Also, as $\sigma$ decreases, we begin to see the introduction of small scale instabilities and a deviation from axisymmetry.

\subsection{Vortex Rings, Co-axial $(\mathrm{SWP0} \times 2)$}

Next we consider the interaction of two impulse aligned vortex rings. The initial position and strength of two sheets is given by

$$
\mathbf{X}=\left(\begin{array}{c} 
\pm 0.5+0.25 \sin \eta \\
(1+0.25 \cos \eta) \cos \xi \\
(1+0.25 \cos \eta) \sin \xi
\end{array}\right) \quad \Gamma=-\eta
$$

This particular parameterization models the vortex rings as perfect toroids, with cumulative circulation spread uniformly over each surface. The regularized motion of an isolated single sheet of this type is not steady, as demonstrated by the non-circular streamlines near the sheet surface in Fig. 8.3; steady motion would only be achieved if the sheet surface were also a streamline. In general, the core dynamics of a vortex ring could be accurately modeled with several SWP0 surfaces, each with different ring to tube radii, all focused around the same vortex core. We present here a model of the large scale motion, using only a single surface to define each vortex ring.

3200 triangles are needed to define each of the initial toroids, as shown in Fig. 8.4(a). Again, throughout the evolution axisymmetry is maintained due to the relatively large $\sigma(=0.2$ scaled with the initial tube diameter). The time step is lowered to $\Delta t=0.005$. The initially trailing ring contracts as it moves through the lead ring, then expands 
as it surrounds the lead ring, leaving a long tail of low-vorticity surface behind as in Fig. 8.4(c). The motion is only somewhat similar to the first visualization of leapfrogging vortex rings [61]. The initial lead ring becomes highly distorted as time progresses, and also leaves behind a short tail which may or may not be pulled into the vortex interaction. Unfortunately, further computation is hindered by the high curvature tail of the initial lead ring, which becomes progressively sharper as time continues. In frame (c) the combination of surfaces require $1.2 \cdot 10^{5}$ triangles to define.

\subsection{Vortex Rings, Offset $($ SWP0 $\times 2)$}

Modeling the interaction of two impulse offset vortex rings, we define the initial position and strength of two sheets as in the previous section, but with a slight offset along the $Y$ axis:

$$
\mathbf{X}=\left(\begin{array}{c} 
\pm 0.5+0.25 \sin \eta \\
(1+0.25 \cos \eta) \cos \xi \pm 0.25 \\
(1+0.25 \cos \eta) \sin \xi
\end{array}\right) \quad \Gamma=-\eta
$$

As in the previous section, the core dynamics of each ring are not well represented with a single surface. The time step is again lowered to $\Delta t=0.005, \sigma=0.2$ relative to the initial diameters, and each initial surface has 3200 triangles.

In this simulation, we again see the trailing vortex ring contract and accelerate as it is forced through the other, as shown in Fig. 8.5. This time, however, the top portion of the trailing ring is forced though first, ahead of the lower portion. This top to bottom discrepancy is exaggerated by the last time frame, producing a distinct "V" in the trailing sheet surface (Fig. 8.5(c)). Calculation to longer time is hindered by the relatively sharp edges spanning the entire circumference of the sheet surfaces (clearly discernible in Fig. 8.5(c)), the time of which requires $1.4 \cdot 10^{5}$ triangles to define. As with the in-line vortex rings, symmetry (though not enforced) is preserved well. 
Fig. 8.6 shows the vorticity distribution at the final time. Clearly vorticity has localized to thin circumferential bands on each surface, leaving a vast majority of the surface area to move as a simple material surface. Finally in Fig. 8.7 we show a cut-away of the final time's mesh skeleton, revealing to some extent the large length scale differences between flat areas and the sharp edges mentioned previously.

\subsection{Vortex Rings, Interlocked $($ SWP0 $\times 2)$}

Modeling a fully 3D interaction of two interlocked vortex rings, we define the initial position and strength of the two sheets as

$$
\mathbf{X}=\left(\begin{array}{c}
0.25 \sin \eta \\
(1+0.25 \cos \eta) \cos \xi \\
(1+0.25 \cos \eta) \sin \xi
\end{array}\right) \quad \Gamma=-\eta
$$

and

$$
\mathbf{X}=\left(\begin{array}{c}
(1+0.25 \cos \eta) \cos \xi \\
0.25 \sin \eta \\
(1+0.25 \cos \eta) \sin \xi
\end{array}\right) \quad \Gamma=-\eta
$$

as depicted in Fig. 8.8(a). As in the previous section, the core dynamics of each ring are not well represented with a single surface. The time step is again lowered to $\Delta t=0.005, \sigma=0.2$ relative to the initial diameters, and each initial surface has 3200 triangles.

The horizontal ring and fluid in its center are propelled upward, causing a fold to develop in the vertical ring's surface, despite stabilizing rotation about its tubular core. Fig. 8.8(b) hints at the formation of this fold, while Fig. 8.8(c) and (d) give an indication of how sharp the fold becomes. At the same time, the vertical ring propagates into the page, creating a similar fold in the horizontal ring. At a later time, in Fig. 8.8(c), significant length scale differentes exist in different parts of the 
sheet, as seen in Fig. 8.8(d): the smallest triangle edges at this time are $4 \cdot 10^{-4}$ of the initial ring radius. Analogous deformations occur for the horizontal ring, which are hidden from view in Fig. 8.8(c). Each sheet in Fig. 8.8(c) has 50,000 triangles, but again calculations cannot realistically proceed in time as the curvature in the fold region is increasing dramatically, requiring so many triangles to resolve that the current simulation proceeds so slowly as to be impractical. 
(a)

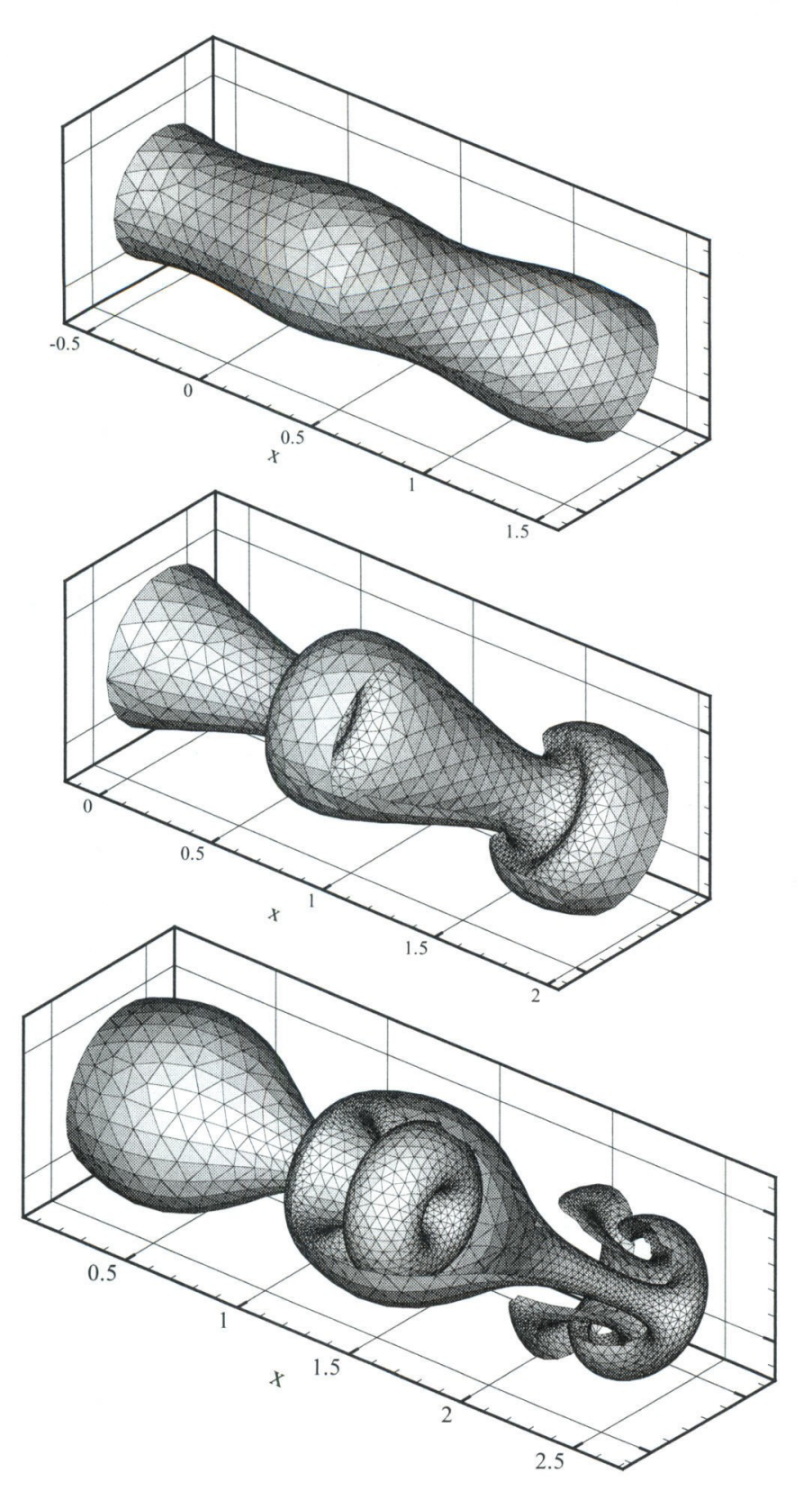

(b)

(c)

Figure 8.1: SWP1 vortex sheet with strength distribution simulating a hollow-core jet with initial $1^{s t}$ mode axial perturbation, $\sigma=0.2$ (relative to initial average diameter). One period calculated, two shown with second in cut-away. Shaded mesh skeleton: (a) $t=0$; (b) $t=0.2$; (c) $t=0.4$; (d) $t=0.6$; (e) zoom of roll-up region in (d); (f) actual smooth surface representation of (e). 


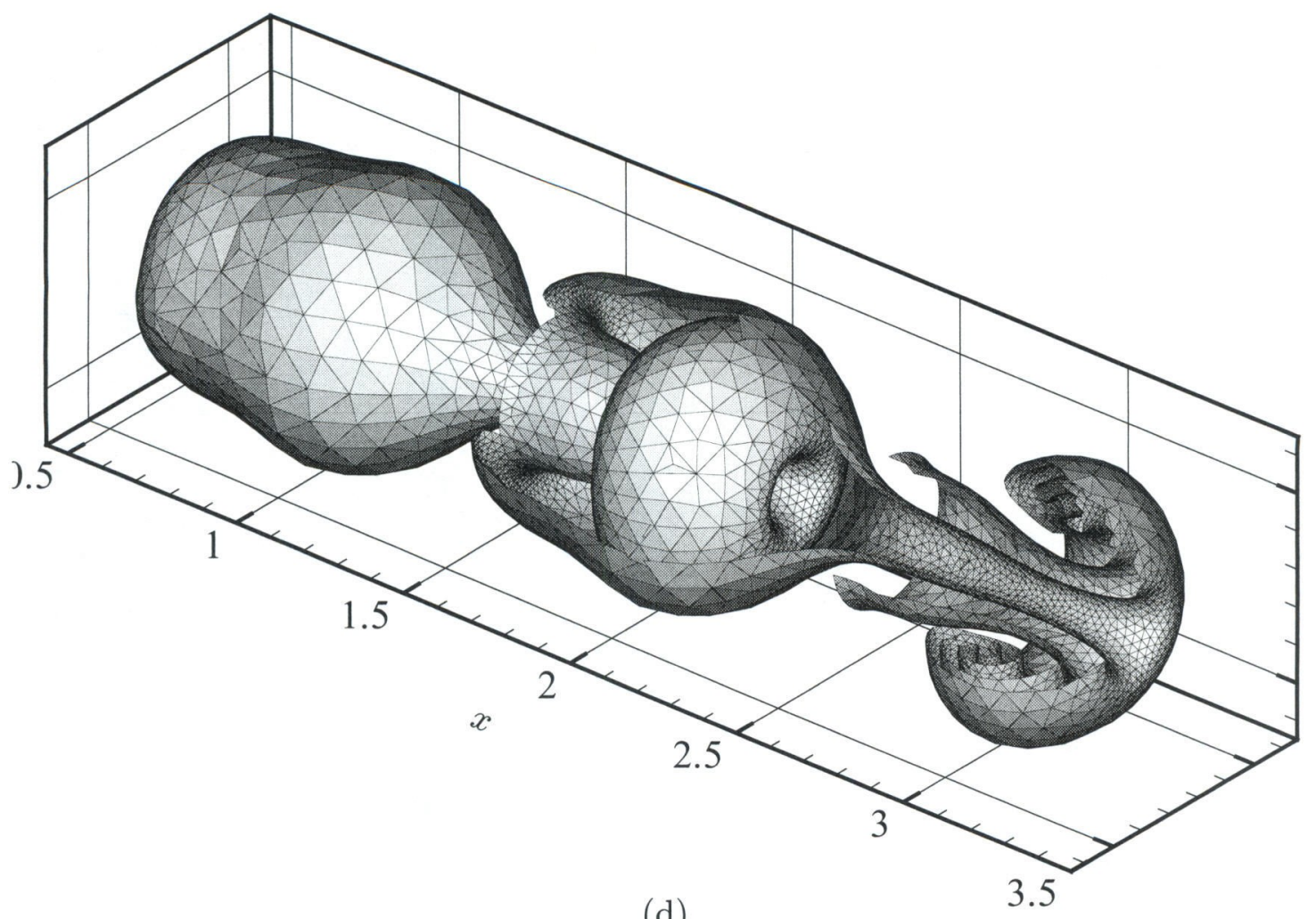

(d)

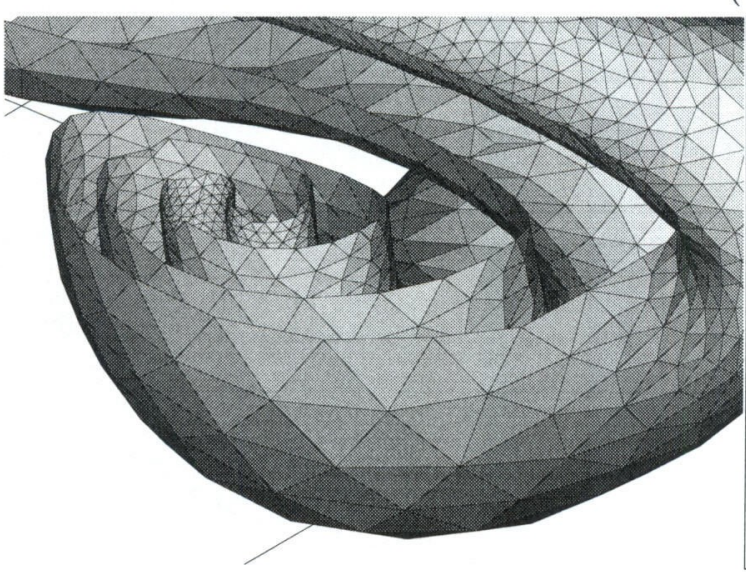

(e)

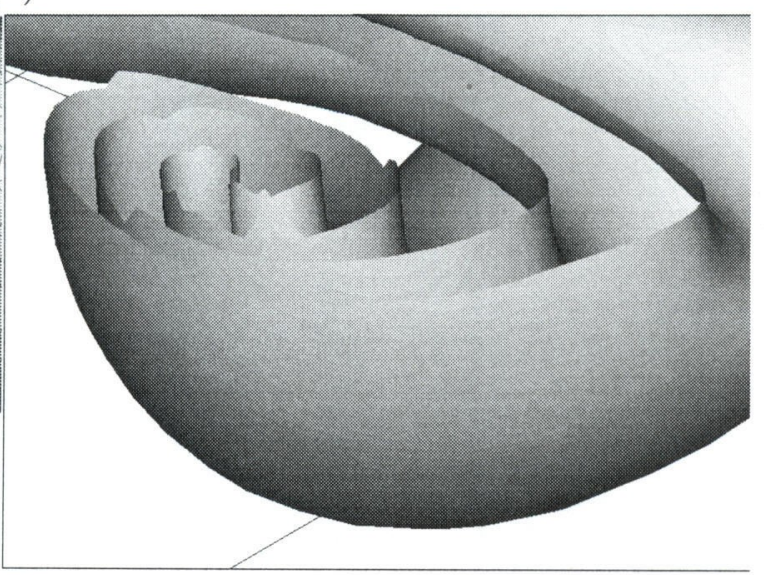

(f)

Figure 8.1: (cont) 
(a)

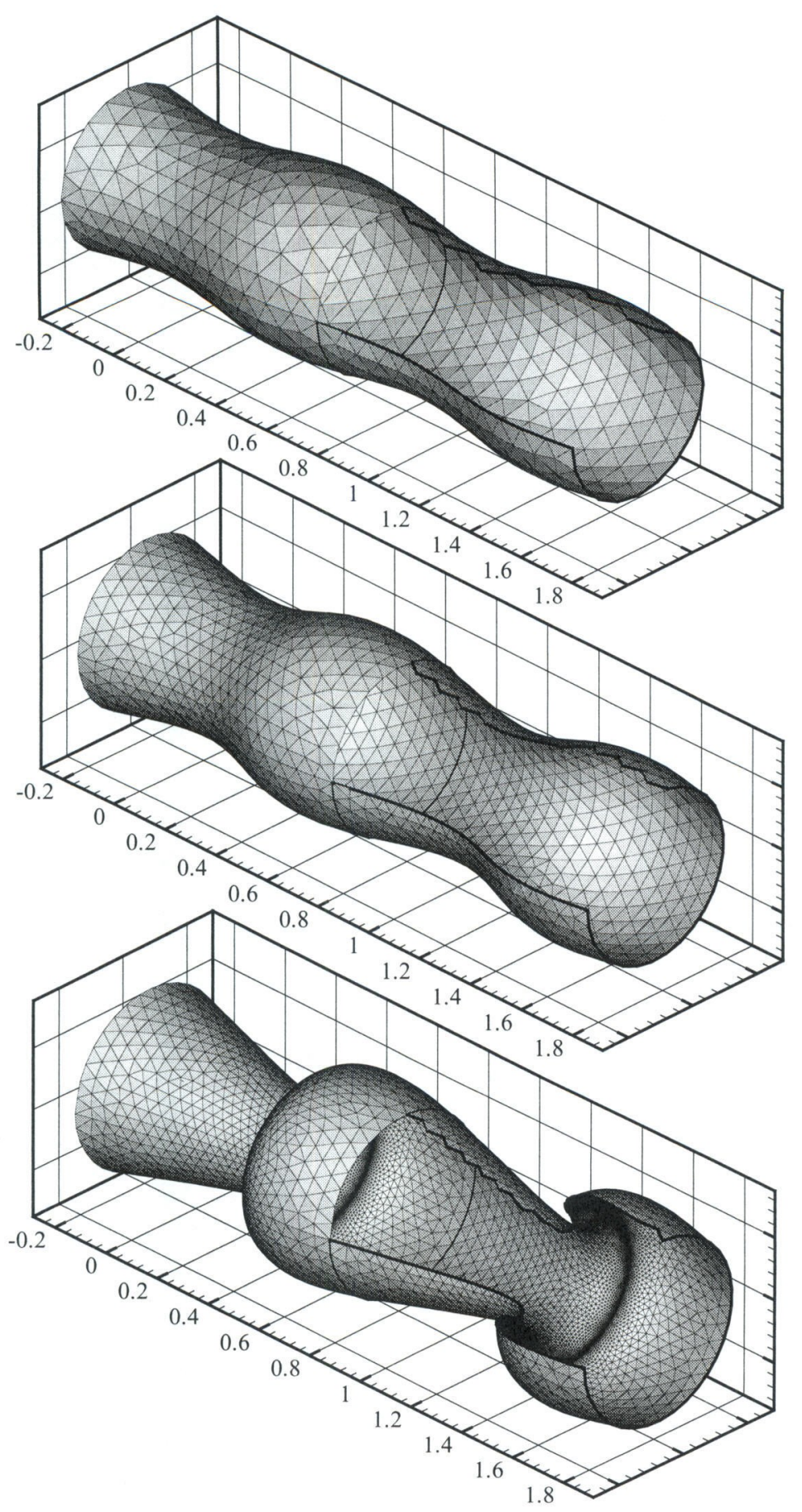

Figure 8.2: Shaded mesh skeleton of SWP1 vortex sheet with strength distribution simulating a hollow-core jet at $t=0.12$. Initial $1^{\text {st }}$ mode axial perturbation. One period calculated, two shown with second in cut-away. (a) $\sigma=0.5$, (b) $\sigma=0.25$, (c) $\sigma=0.0625$. 


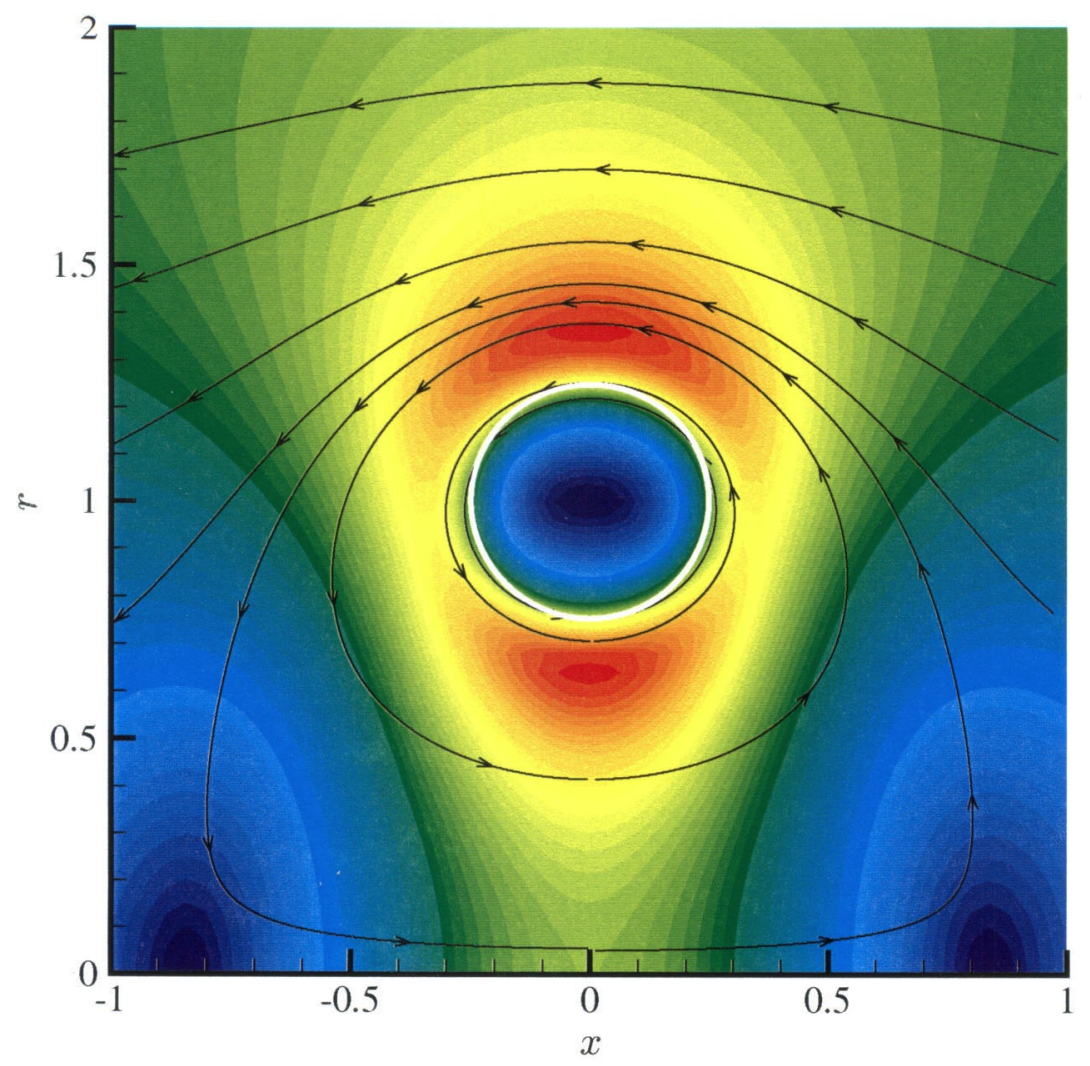

Figure 8.3: Slice through the vortex ring model, depicting instantaneous $(t=0)$ velocity field in a frame moving with the vortex ring. The axes are radial distance $r$ from the center of the ring, and distance $x$ parallel to the impulse vector. Color contours represent the velocity magnitude, from zero (dark blue) to 1.8 times the propagation velocity (red); black lines are instantaneous streamlines; the white circle is the vortex sheet surface. 
80

(a)
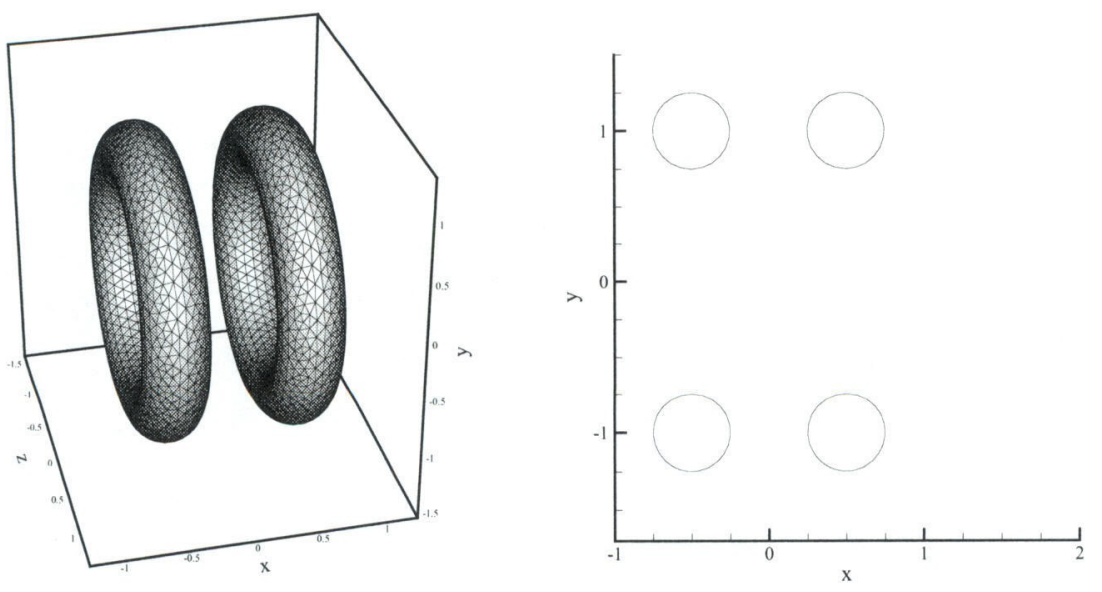

(b)
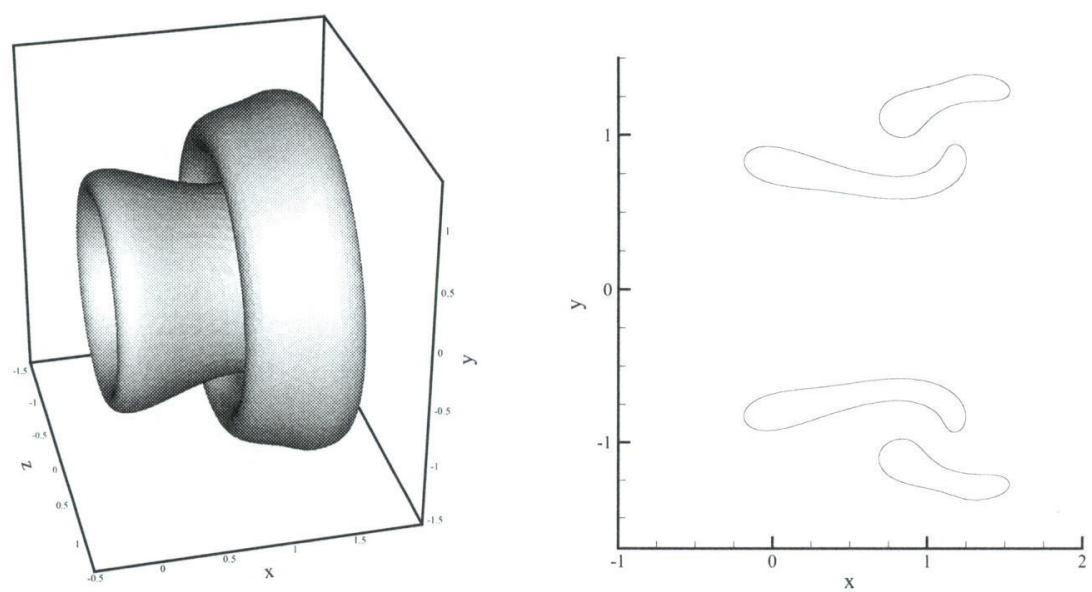

(c)
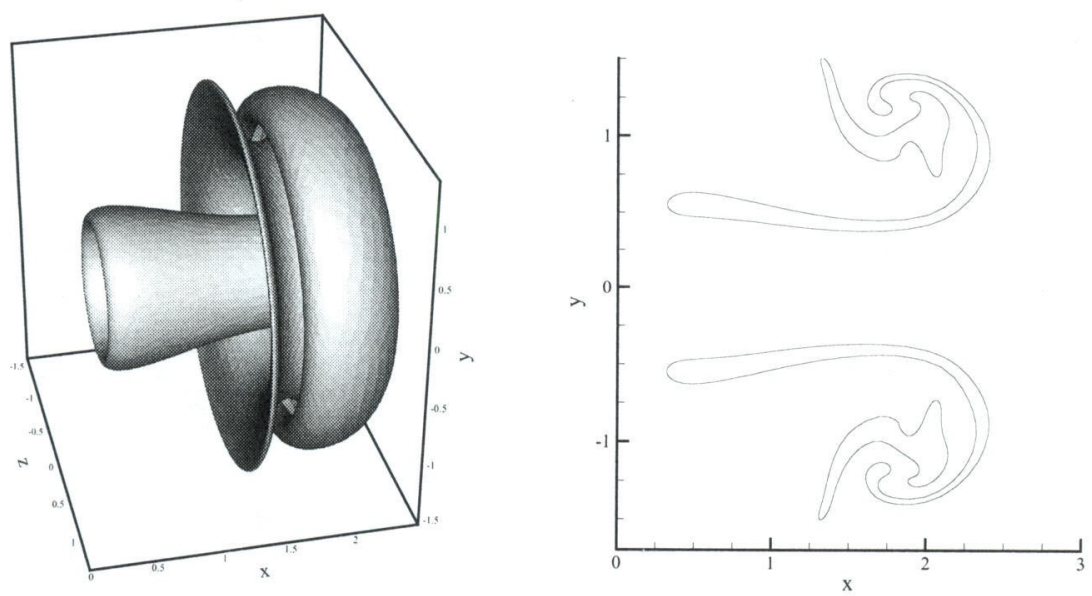

Figure 8.4: Shaded mesh skeleton of hollow-core vortex rings (SWP0) with in-line impulse vectors, $\sigma=0.2$ (relative to initial tube diameters). Ring to tube radius ratio is 4 . Right side is a vertical slice through the ring centers; (a) $t=0$, (b) $t=0.44$, (c) $\mathrm{t}=0.88$. 
(a)
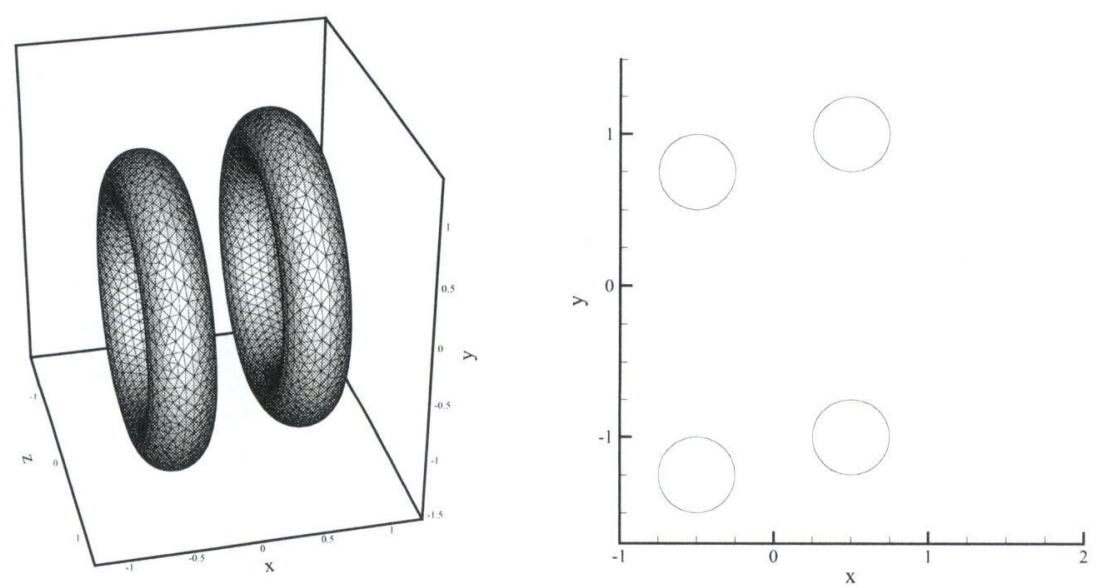

(b)
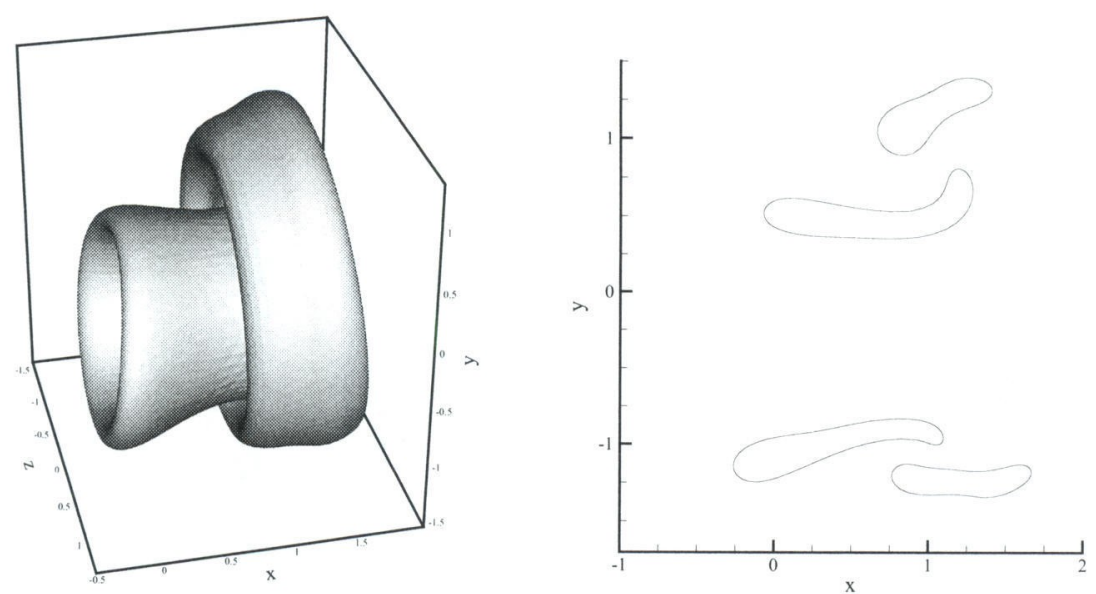

(c)
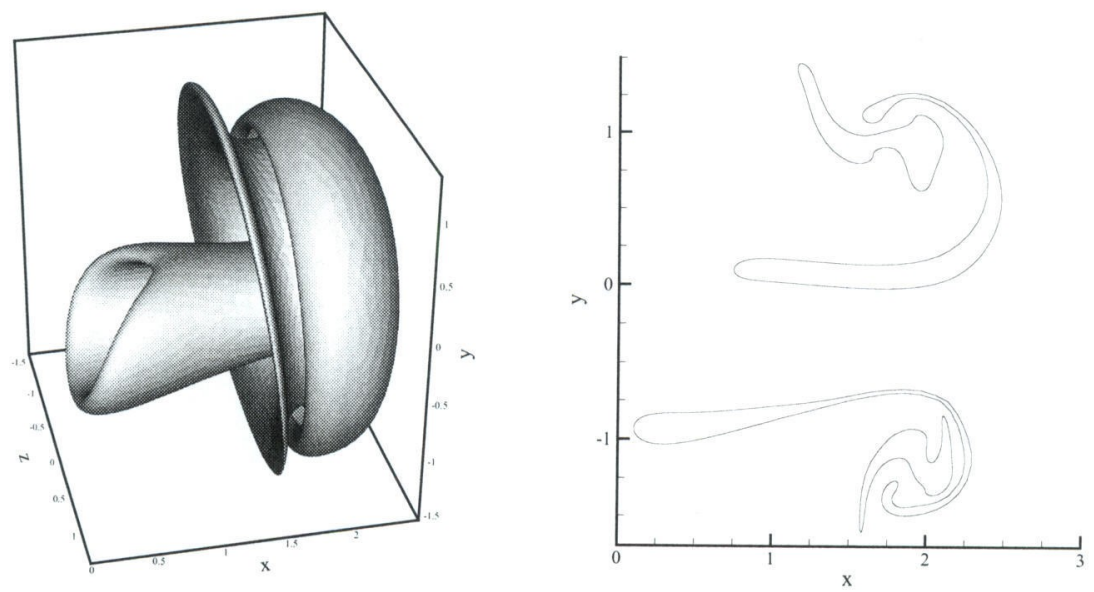

Figure 8.5: Shaded mesh skeleton of hollow-core vortex rings (SWP0) with offset impulse vectors, $\sigma=0.2$ (relative to initial tube diameters). Ring to tube radius ratio is 4 . Right side is a vertical slice through the ring centers; (a) $t=0$, (b) $t=0.44$, (c) $\mathrm{t}=0.88$. 


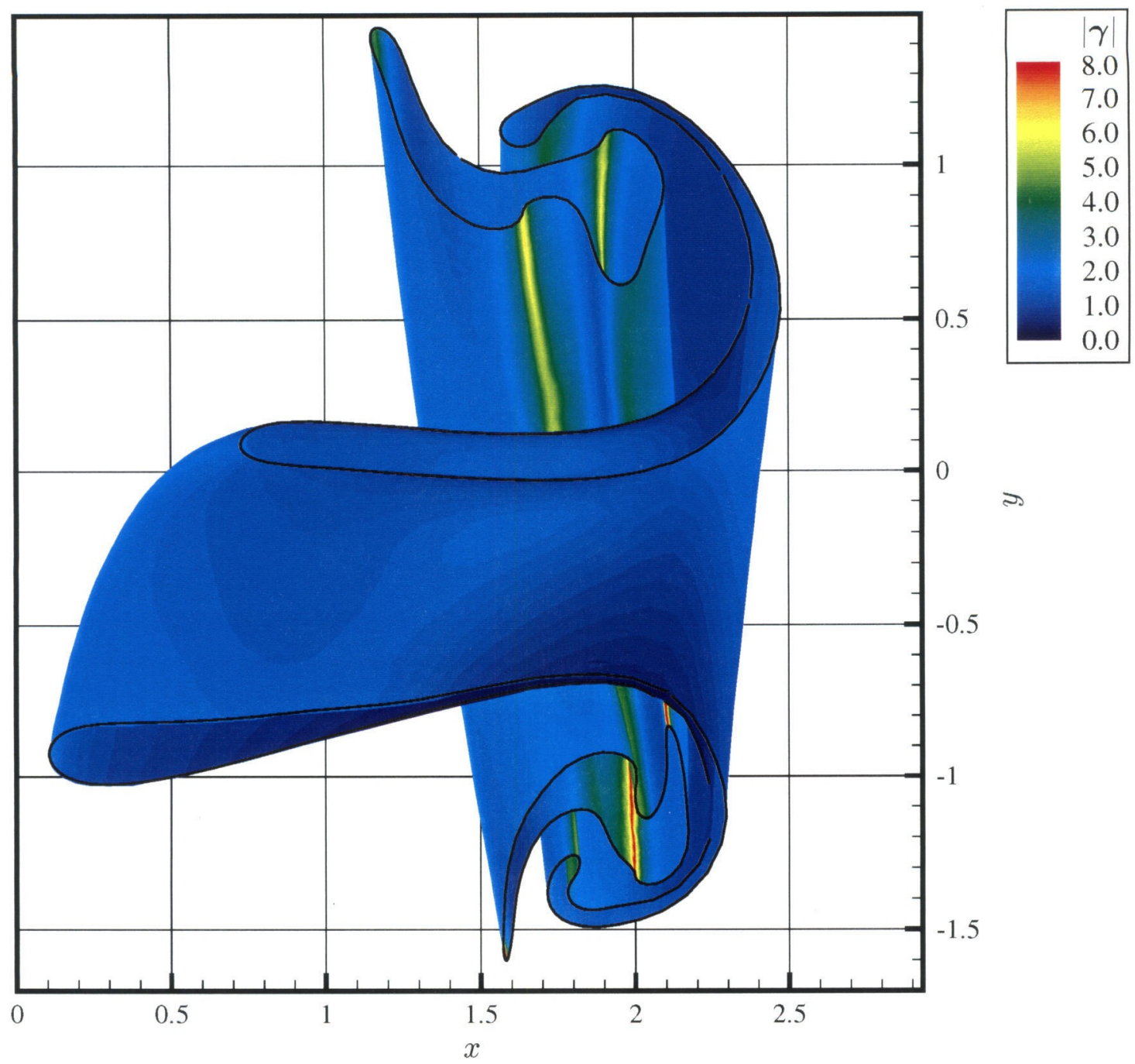

Figure 8.6: Time slice corresponding to Fig. 8.5(c), colored by sheet strength $|\boldsymbol{\gamma}|$. Vorticity has localized to thin bands on each surface. 


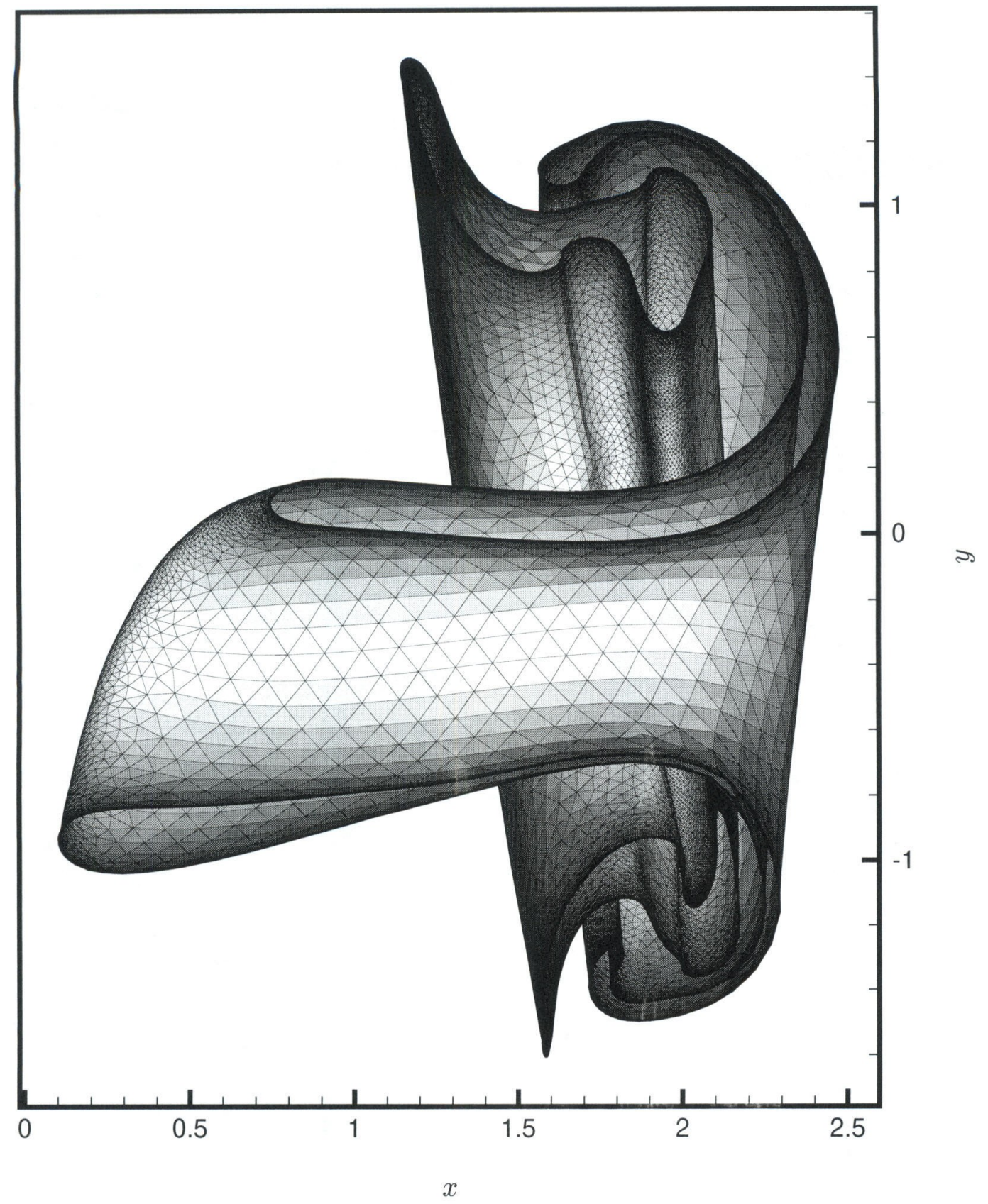

Figure 8.7: Mesh skeleton corresponding to Fig. 8.5(c), detailing triangulation. Note the high curvature fold developing in the bottom portion of the surface. 


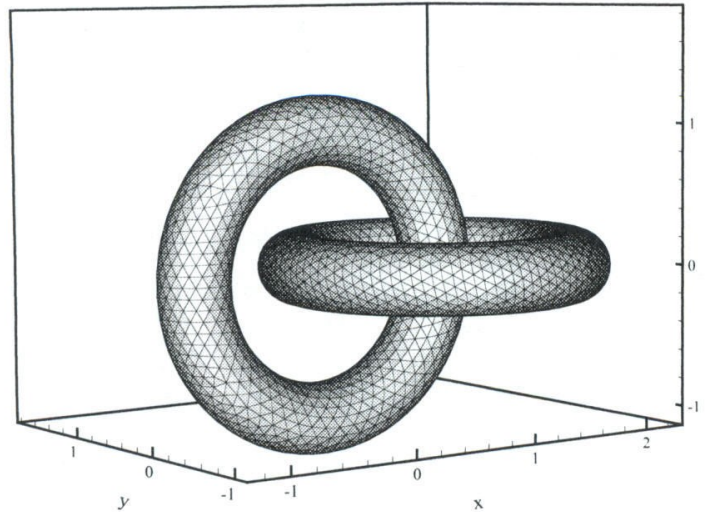

(a)

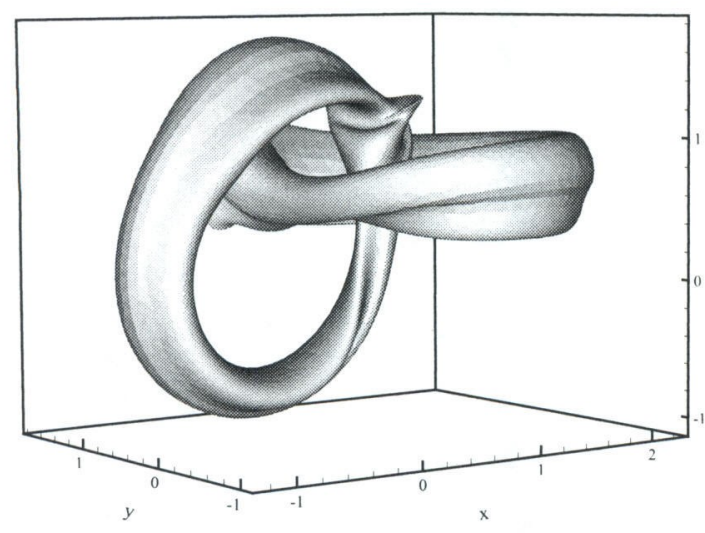

(c)

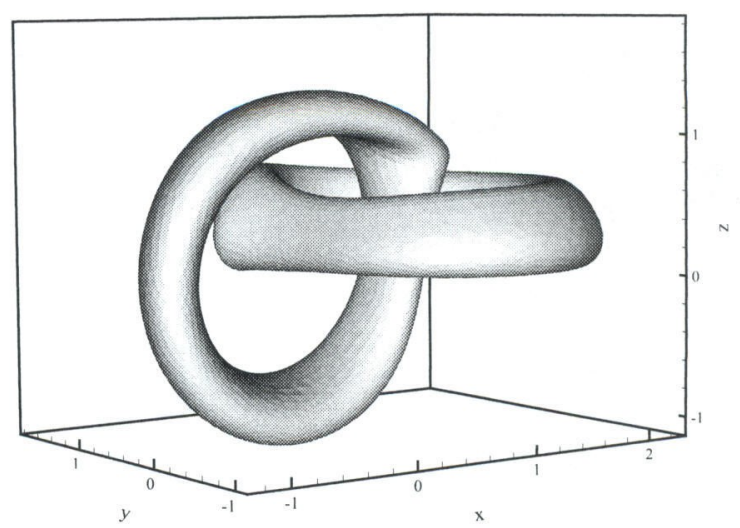

(b)

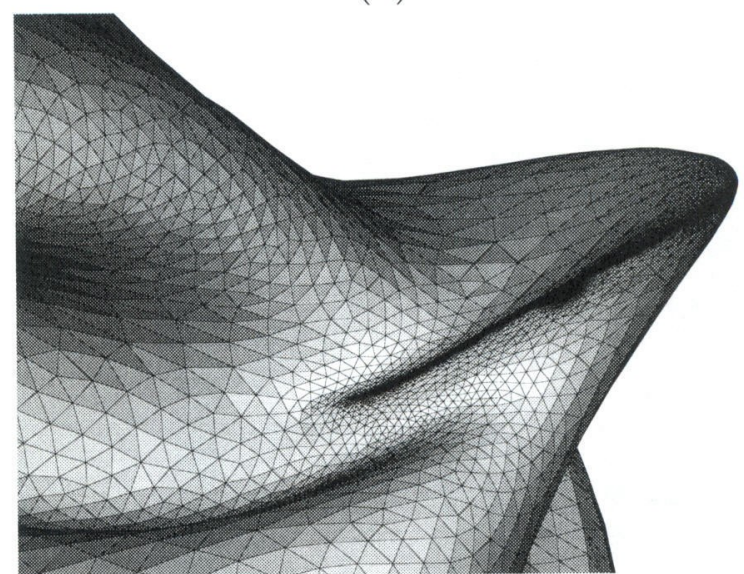

(d)

Figure 8.8: Shaded mesh skeleton of interlocked hollow-core vortex rings (SWP0) with perpendicular impulse vectors. ( $\sigma=0.2$ relative to initial tube diameter; ring to tube radius ratio is 4.) Vertical ring impulse points left and into the page, while horizontal ring impulse points up. (a) $\mathrm{t}=0$, (b) $\mathrm{t}=0.28$, (c) $\mathrm{t}=0.54$, (d) zoom of pinched region in (c). 


\section{Chapter 9 Shear Layer Application}

As implied in Chapter 1, the spatially evolving, perturbed planar shear layer is a configuration ideally suited for the vortex sheet model. The free shear layer is a classic fluid mechanics problem with applications in many areas. Particularly interesting in the shear layer is the development of streamwise vorticity after the primary Kelvin-Helmholtz instability forms, from initial conditions dominated by spanwise vorticity. We choose a particular $3 \mathrm{D}$ initial perturbation, not to model a specific experimental condition, but rather to characterize the behavior of a general spanwise and streamwise perturbations. At our disposal we have the doubly periodic SWP2 surface, and the smoothing parameter $\sigma$.

The initial perturbation implemented is as follows:

$$
\begin{gathered}
\mathbf{X}=\left(\begin{array}{c}
\xi / 2 \pi \\
\eta / 2 \pi \\
(\sin \xi+\sin \eta) / 10
\end{array}\right) \\
\Gamma=-\xi / 2 \pi-(\sin \xi+\sin \eta) / 10 .
\end{gathered}
$$

This initial condition is a true linear perturbation in the sense that the sheet strength is perturbed in addition to the geometry as designated by a linear perturbation analysis of the planar vortex sheet; it is not, however, a single normal mode but rather a combination (see Chapter 11 for details of the linear analysis). The initial geometry is shown in Fig. 9.1(a); fluid above the sheet moves out of the page and to the left (along the negative $x$ axis), while fluid below moves in the opposite direction. The smoothing parameter is $\sigma=0.1$ and the perturbation magnitude is 0.2 , both relative to the (unit) period length. The motion of only one of the four periods depicted in Fig. 9.1 is calculated. Extra periods, as well as two vertical plane cuts, are shown for visual clarity. 


\subsection{Observations}

At $t=1.0$ in the simulated shear layer evolution, the primary spanwise rollers have clearly formed (Kelvin-Helmholtz instability), and the spanwise perturbation has grown considerably, as shown in Fig. 9.1(b). Note that the sheet boundary in Fig. 9.1(a) lies in constant $x$ and constant $y$ planes; such is not the case at later times, as the boundaries have moved in a Lagrangian sense. The roll-up as seen by the two plane cuts is quite similar to the roll-up of a purely $2 \mathrm{D}$ shear layer which, for reference, is depicted in Fig. 10.3. At the time in Fig. 9.1(c) we see the onset of streamwise roll-up, as evidenced by the streamwise fold, indicating that some of the initially spanwise dominated vorticity has shifted to streamwise. Fig. 9.1(d), though complicated to view, depicts regions where the vorticity magnitude is greater than a threshold cut-off. One can see two distinct regions of vorticity: (1) the core of the perturbed spanwise rollers with vorticity oriented primarily in the spanwise direction (blue arrows) and (2) the connected looping structure containing the streamwise vorticity, alternately pointing upstream and downstream (red arrows). These looping structures bear a strong resemblance to the horseshoe vortices known to form in the plane boundary layer, and the shear-generated hairpin vortices seen in homogeneous turbulent computations. Fig. 9.2 depicts the sheet at the same time as Fig. 9.1(d), but is color-contoured with the local vorticity magnitude, $|\boldsymbol{\gamma}|$, revealing not only the streamwise development of vorticity, but also scarcity of vorticity elsewhere, besides in the Kelvin-Helmholtz core. Indeed it would seem at this late time that representation of the entire sheet surface is redundant, as the vorticity is so finely concentrated into filament structures. Also shown in Fig. 9.2 are select vortex lines, visibly collecting in the streamwise concentration of vorticity between the primary rollers.

Fig. 9.3(a) shows an approximation of the circulation held by the spanwise and streamwise vortices as a function of time. The streamwise intensity increases linearly with time after $t=1.0$, while the spanwise circulation hits a local maximum near the end of the simulation, indicating that the two primary structures holding vorticity have not yet reached a steady-state. The circulation ratio is shown in Fig. 9.3(b), 
ranging from 7 to 9 in the time calculated, but apparently will reach values less than 7 at longer times. Circulation is calculated by closed loop path integration, the contour of which approximately surrounded each vorticity concentration independently.

To reveal the structure of the sheet at the final time, $t=1.8$, we examine plane cuts through the surface at constant $x$ and $y$, as shown in Fig. 9.4 and Fig. 9.5. Fig. 9.4 nicely reveals the extent of the Kelvin-Helmholtz roll-up and also, particularly in frames (c) and (d), the core deformation otherwise hidden beneath the outer layers of

the sheet. Visible in frame (b), the developing streamwise vorticity is generally aligned with the $x$ axis, but not precisely. In Fig. 9.5 the Kelvin-Helmholtz core structure is dissected further with plane cuts of constant $x$ through the core. In frame (c), the core is clearly revealed to contain axial vorticity itself, due to deformation, as it passes into and out of a plane of constant $x$. Fig. 9.6 further reveals the spiral structure visible in Fig. 9.5(c). Note in the close-up Fig. 9.6 (a) and (b) the Kelvin-Helmholtz core is deformed upward, as it is 'pulled' by the opposite-signed streamwise vortices just above it.

\subsection{Other Experiments}

Lasheras \& Choi [30] investigated experimentally a spatially evolving shear layer with imposed periodic spanwise and streamwise perturbations. Their shear layer was generated with two laminar streams of water at different speed $(3.4$ and $1.5 \mathrm{~cm} / \mathrm{s})$ joining at the termination of a splitter plate. Upstream conditions were carefully controlled such that without any forced disturbances, the free shear layer extending from the plate developed in an effectively 2D manner for several Kelvin-Helmholtz wavelengths past the splitter plate termination line. Two periodic perturbation modes were introduced to the free shear layer with physical perturbations of the splitter plate: (a) vertical corrugations visible when viewed from downstream (i.e., normal to the plate), and (b) splitter termination variations visible when viewed from above the plate (i.e., in the plane of the plate). The experiments were run at $\operatorname{Re}=45$ based on the velocity difference of the two streams and the momentum thickness at the plate 
termination line. Their visualization was created by spotlight induced fluorescence from fluorescein particles in the lower stream.

With the two independent splitter plate geometry variations, Lasheras \& Choi were able to test the effects of several different initial conditions. For the specific case shown here, the splitter plate was configured with perturbations in the plane of the plate, as visible on the left edge of Fig. 9.7(a). In this experimental visualization, flow is from left to right. The lower, slow stream has the appearance of a solid 3D structure when flashed with the spotlight from above. The primary Kelvin-Helmholtz rollers are hidden from direct view, but their position can be inferred from the developed streamwise vorticity. The developed streamwise vorticity is evidenced by the rolling sides of the tongue-like structure, particularly above the third spanwise roller.

Despite differences in the exact conditions creating the shear layer, and of course the presence of viscous effects in the experiment, there is a remarkable similarity between the calculations done in this chapter and the experiment, as evidenced by Fig. 9.7(b), which is a $3 \times 3$ periodic grid of the calculation presented in Fig. 9.1(c) at time $t=1.8$. The doubly periodic simulation is particularly similar to the flow surrounding the third roller in Fig. 9.7(a). Lasheras \& Choi do point out that for many different perturbations they could introduce (in plane and normal to the splitter plate), the subsequent introduction of streamwise vorticity are qualitatively the same. Numerical simulations by Ashurst and Meiburg [4] model the experimental shear layer with inviscid vortex filaments producing very similar qualitative results as well, such that even at these low Reynolds numbers $(\approx 100)$, inviscid vortex methods are suitable models of the true, viscous shear layer.

With our initial condition, the source of streamwise vorticity is clear: the relative motion of the sheet surface between the Kelvin-Helmholtz rollers has a spanwise dependence. A portion of the sheet which is initially between two rollers is alternately pulled towards one roller and then the other as the span is traversed. As vortex lines move with the sheet, they approach each other in the surface shearing region between the rollers where the spanwise variation is greatest. This characterization of the onset of streamwise vorticity is essentially the same as that given in [30] and is particularly 
well revealed by the left-most sheet boundary of Fig. 9.7(b): the sheet boundary, a material line, is seen to alternately drift towards and away from the left-most roller. Also quite apparent from Fig. 9.1(c) is that the streamwise alignment of vorticity occurs at a time scale much greater than that of the primary Kelvin-Helmholtz rollup: the streamwise vortices are just beginning to spiral while the primary rollers already have several turns. 
(a)

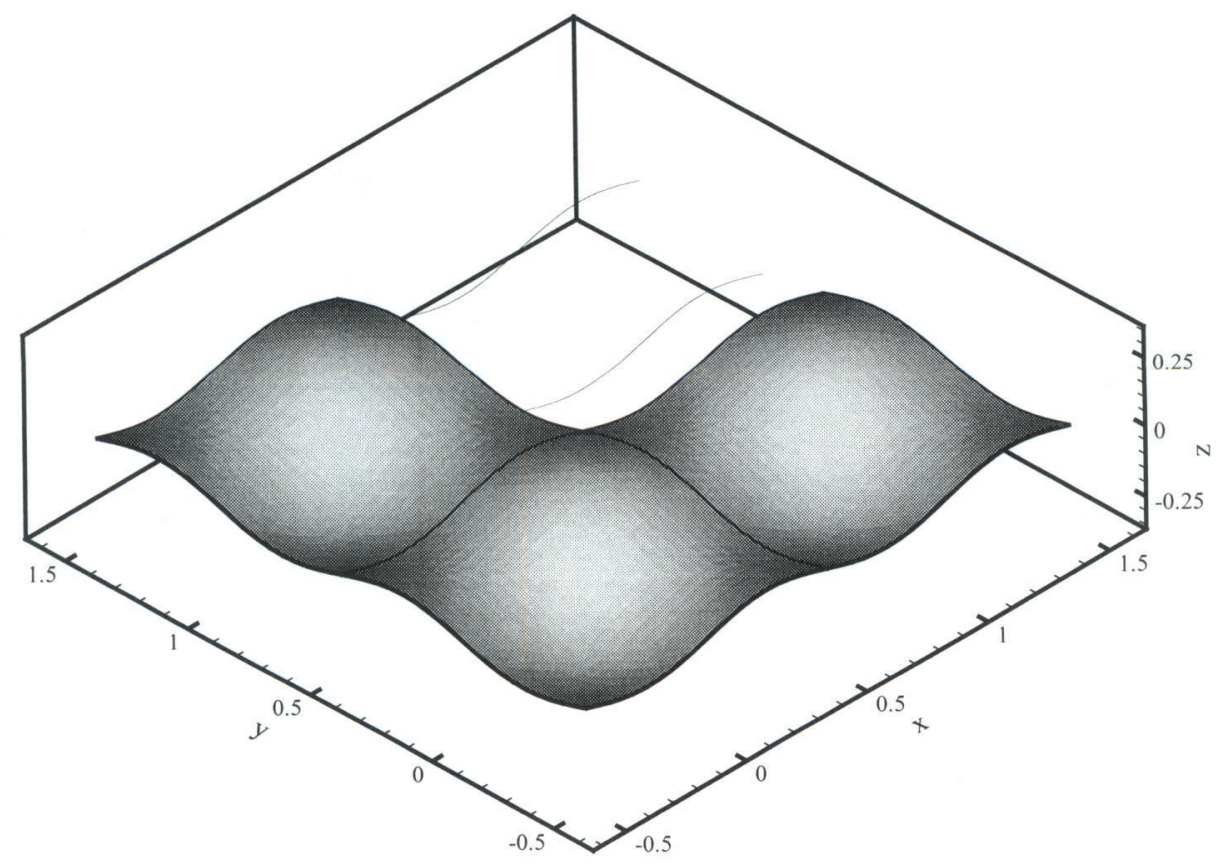

(b)

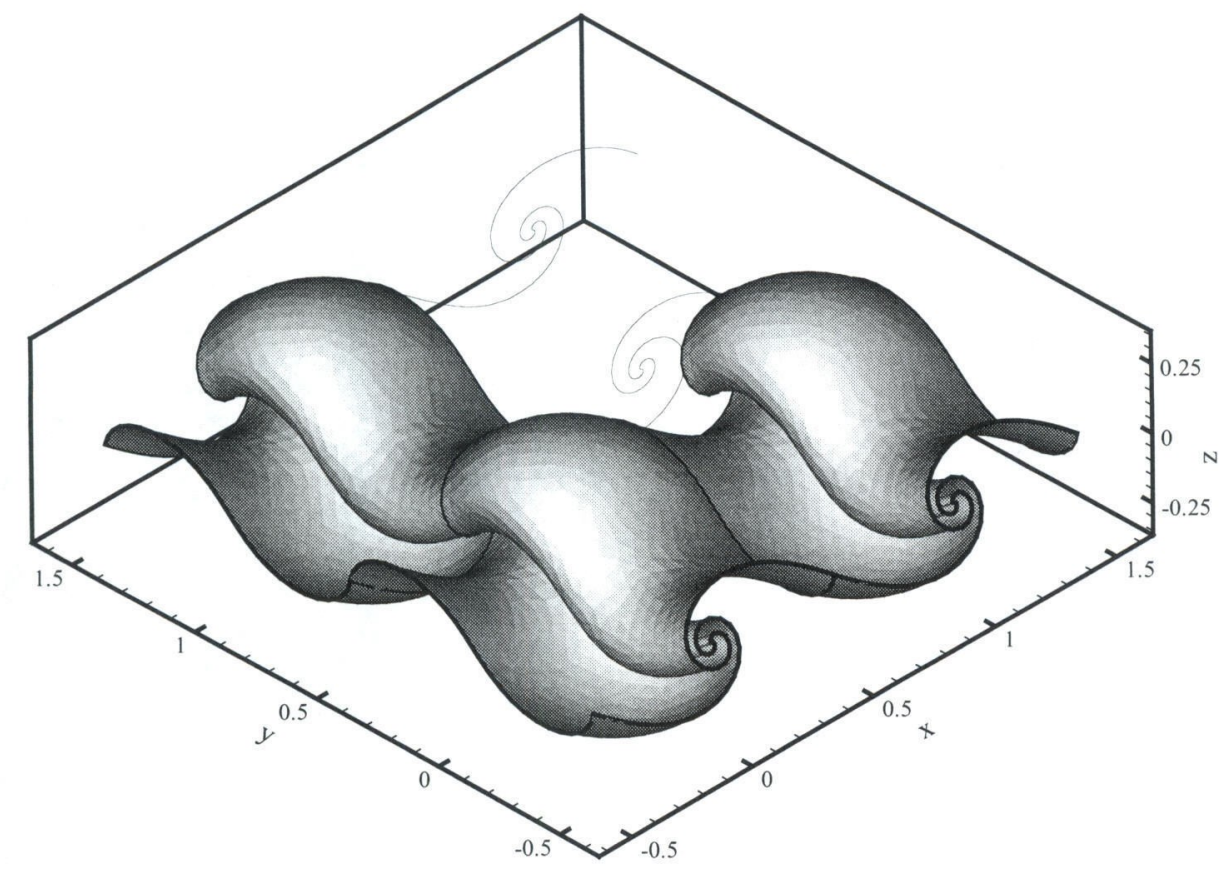

Figure 9.1: Roll-up of a perturbed shear layer (SWP2). One period calculated, three shown shaded, one shown with two vertical slices; (a) $t=0$, (b) $t=1.0$, (c) $t=1.8$; (d) only triangles with vorticity magnitude over a threshold are plotted, revealing the connectivity of the streamwise vorticity. Vortex lines follow the arrows: spanwise (blue), streamwise (red). 
(c)
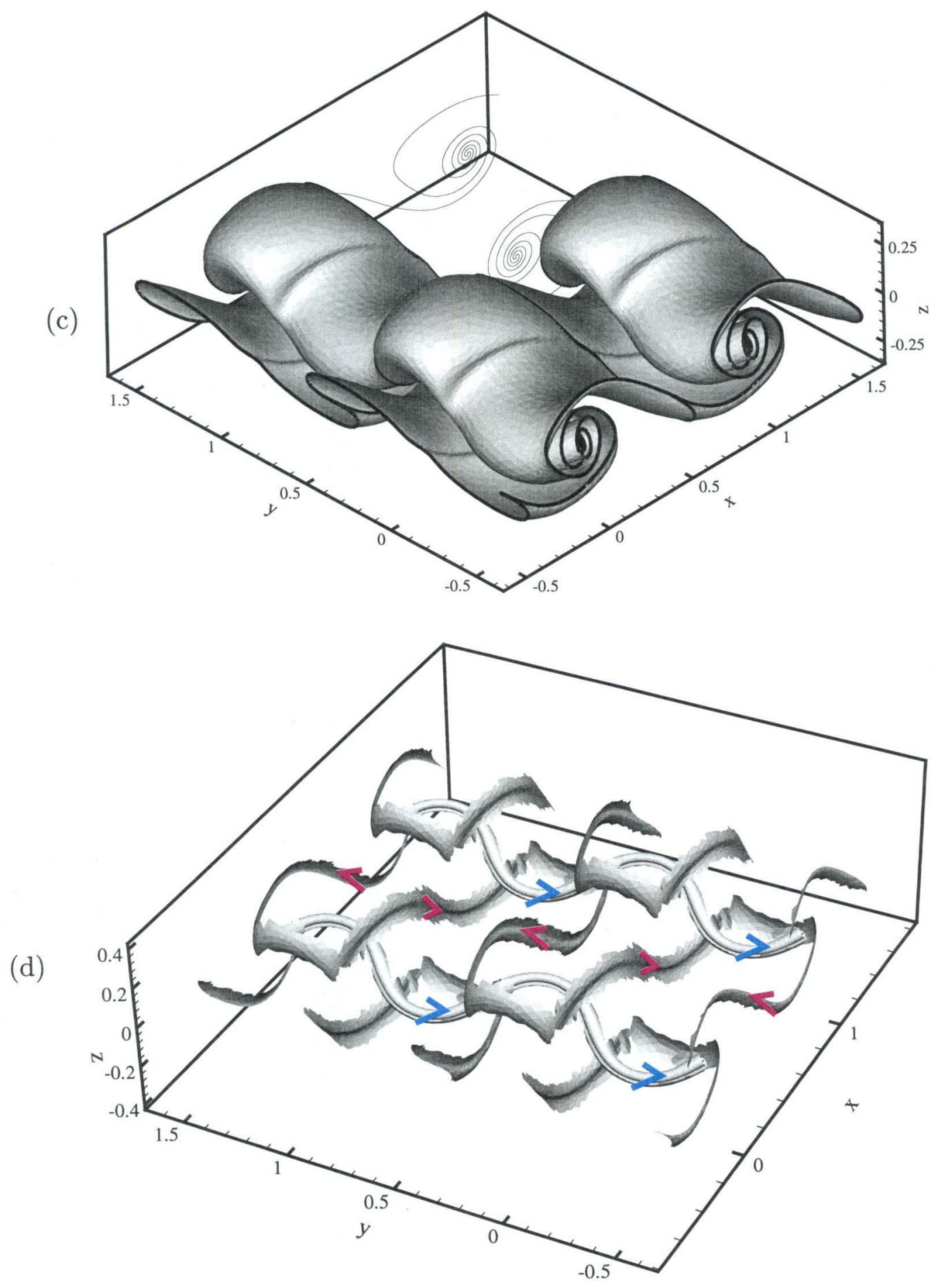

Figure 9.1: (cont) 


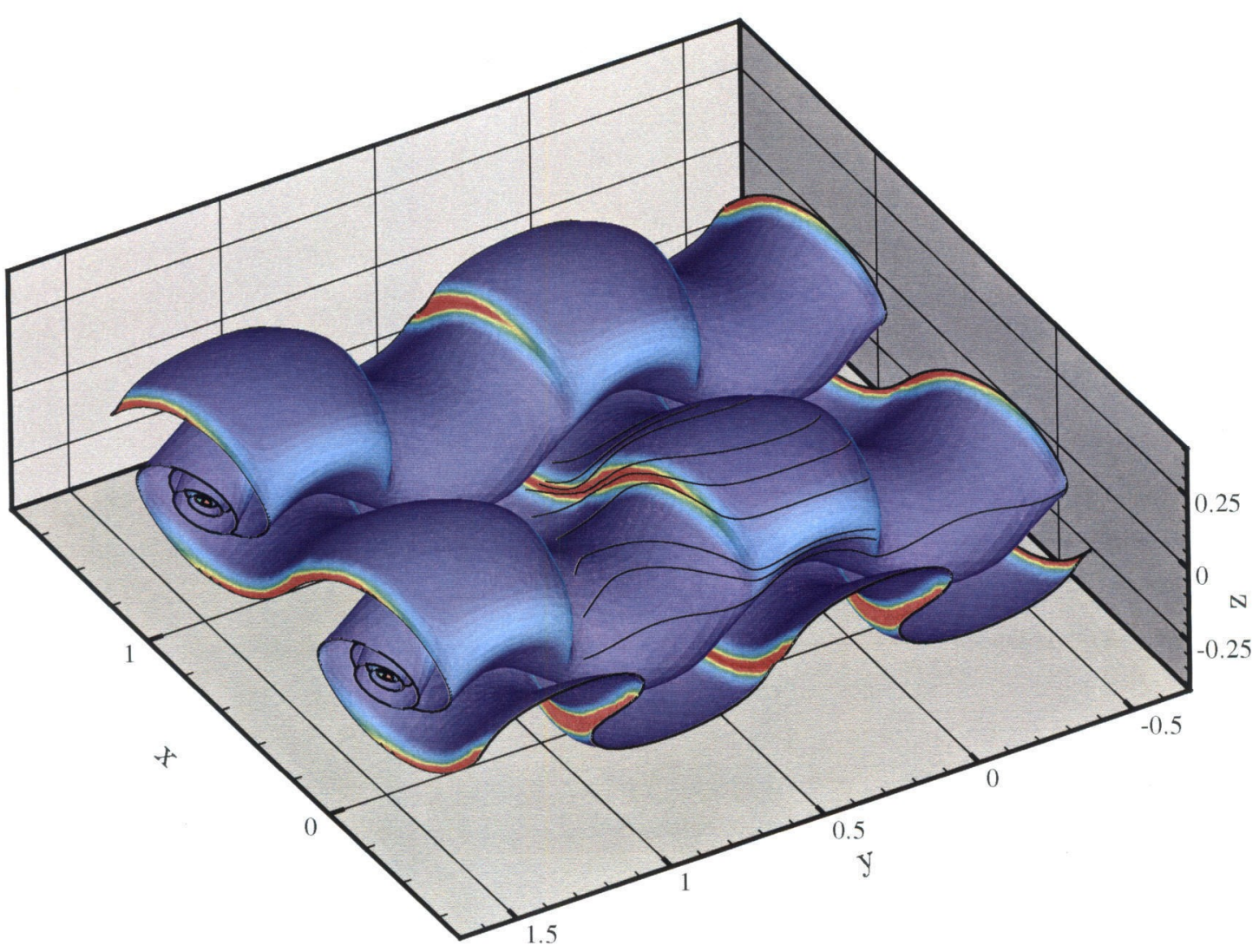

Figure 9.2: Different viewing angle of Fig. 9.1(c), revealing sheet strength $|\gamma|$ and vortex lines. Vorticity has localized to streamwise loops (red) and spanwise rollers (red, hidden), leaving a majority of the sheet surface with little vorticity (blue). 
(a)

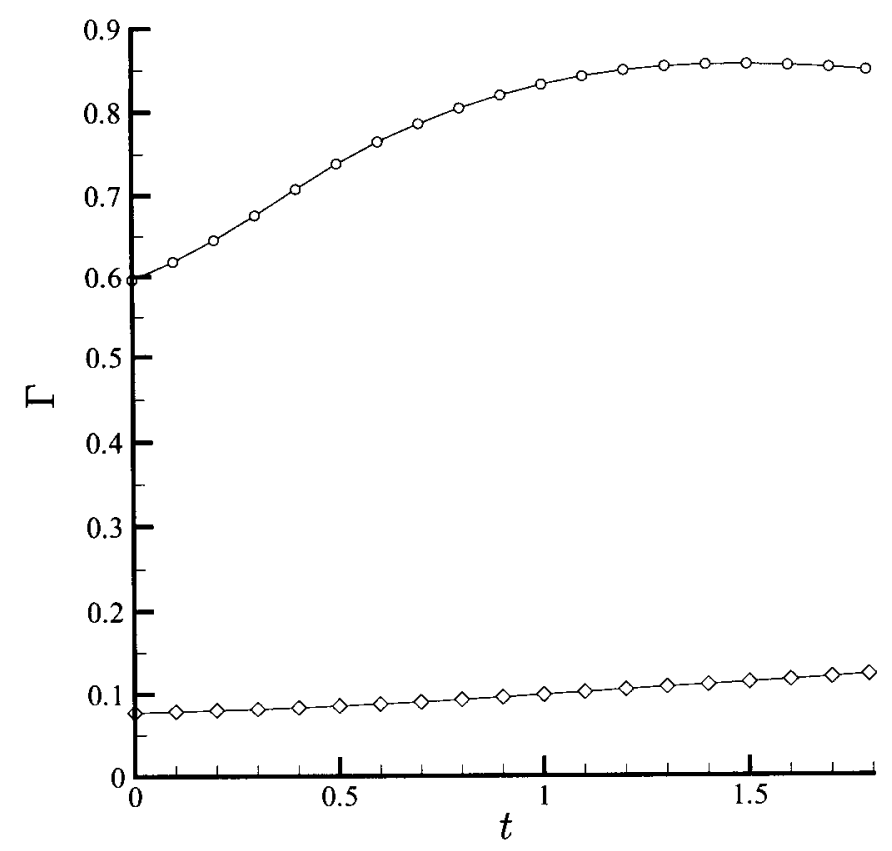

(b)

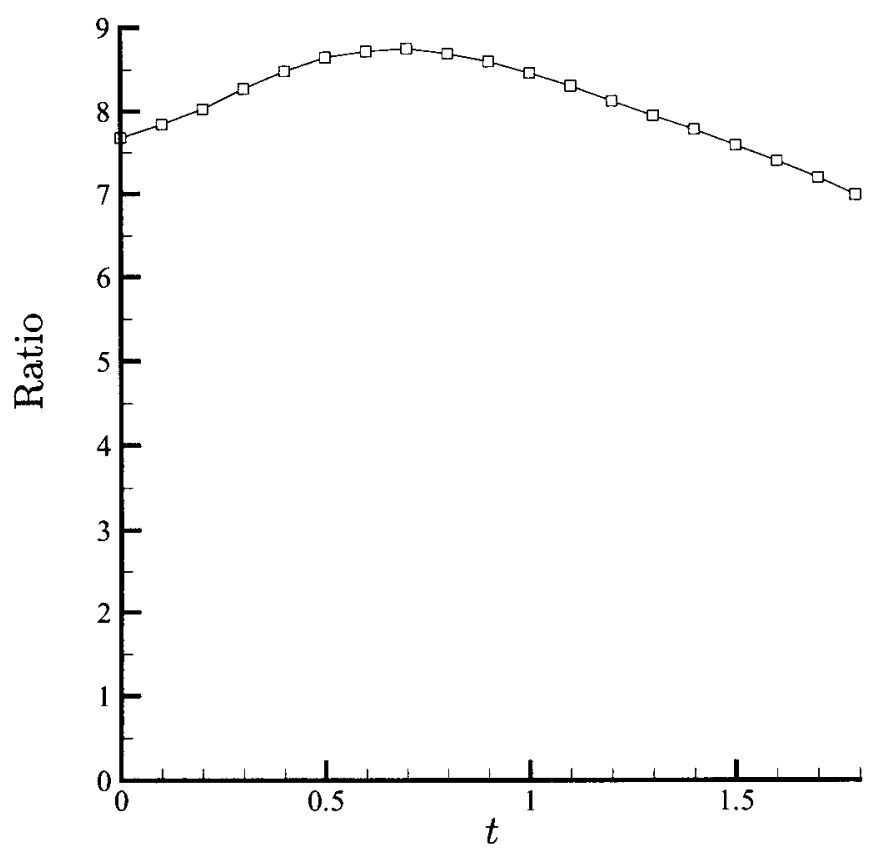

Figure 9.3: Approximate circulation (a) held in the spanwise (o) and streamwise $(\diamond)$ vortices as a function of time; (b) ratio of the spanwise to streamwise circulations. 
(a)

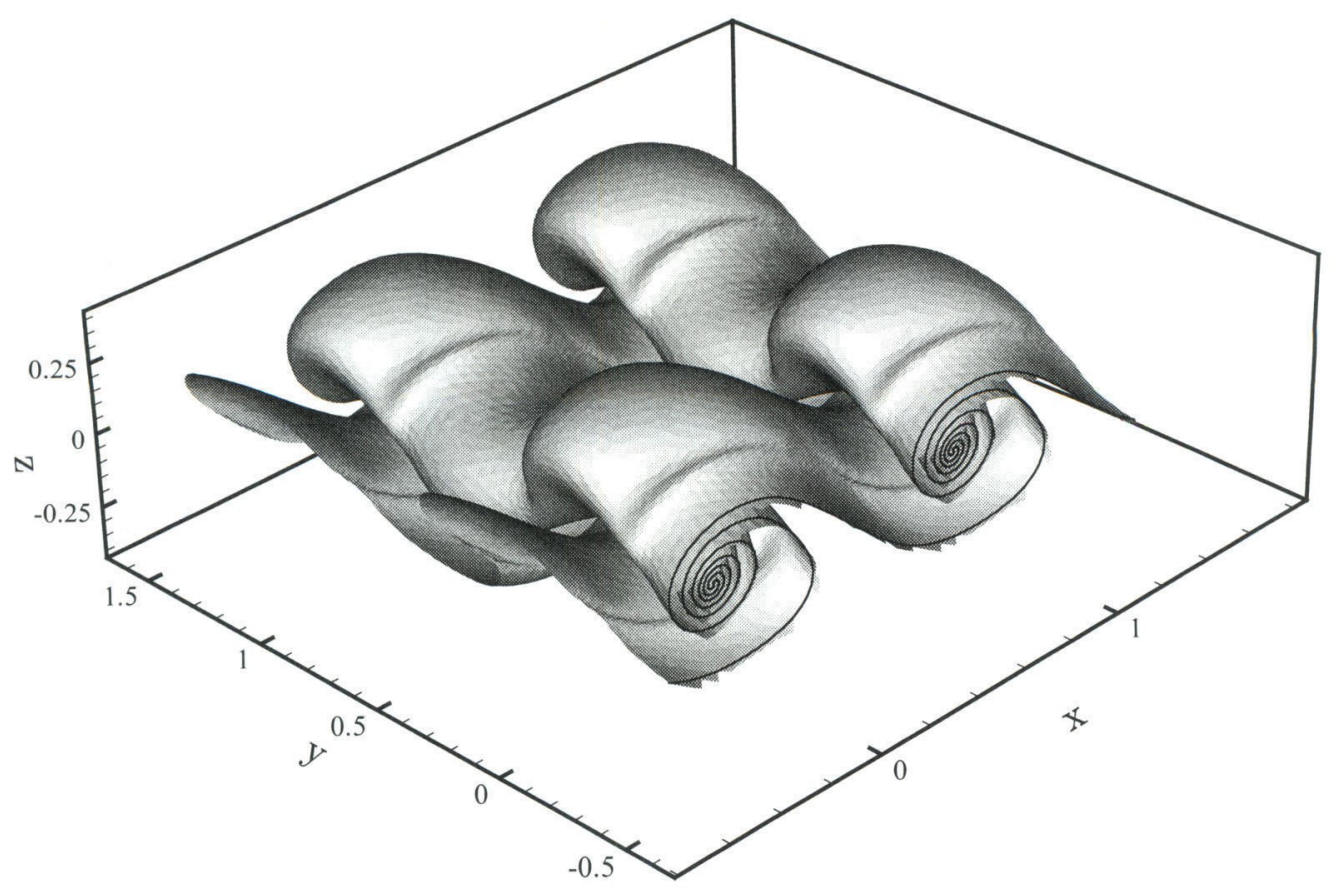

(b)

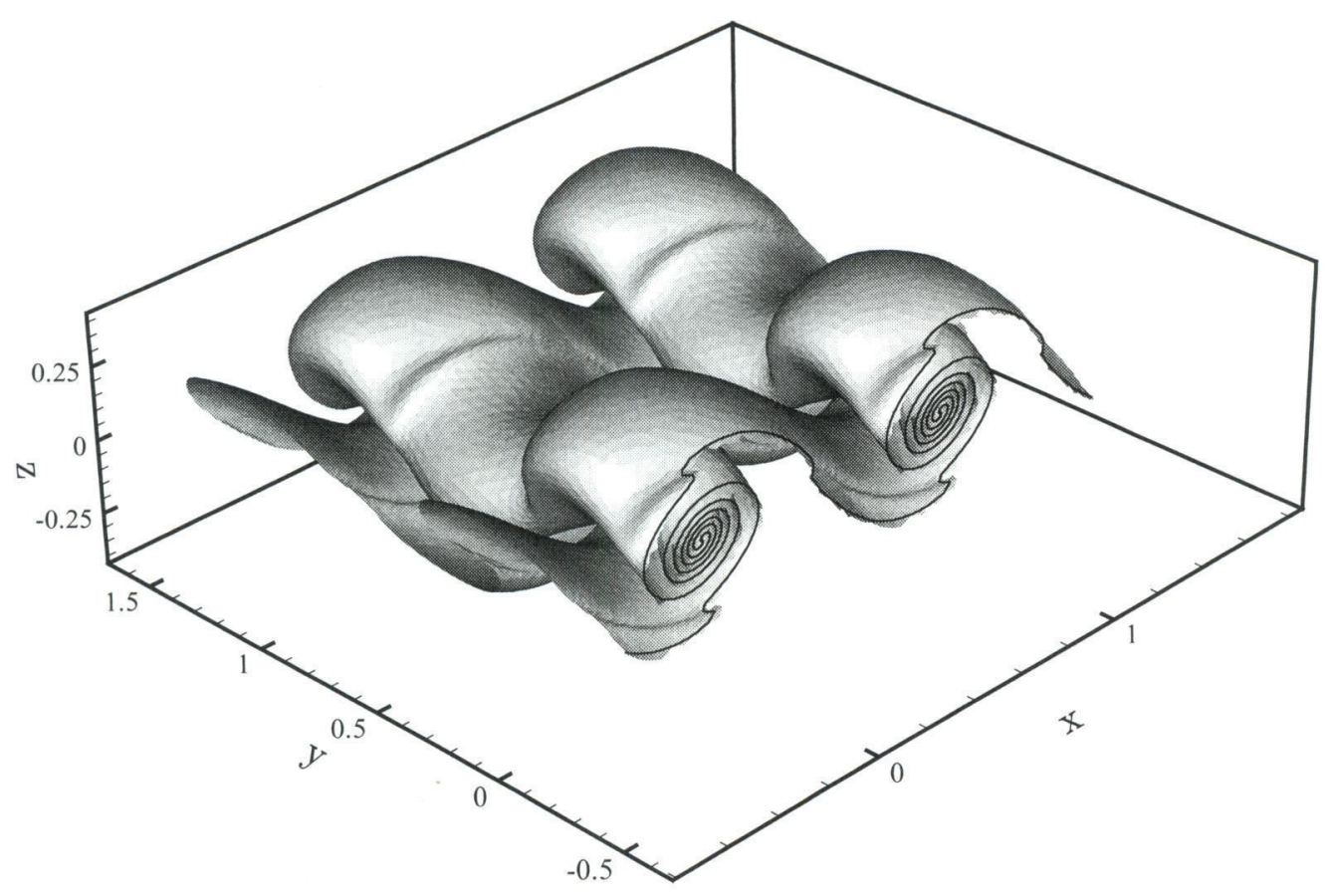

Figure 9.4: Variation of the Kelvin-Helmholtz roll-up with span at the final computed time, $t=1.8$. (a) $y=-0.125$, (b) $y=0$. In (b), the developing streamwise vorticity is not uniformly parallel to the $x$ axis, as implied by the spanwise deviation of the fold from the planar cut. 
(c)

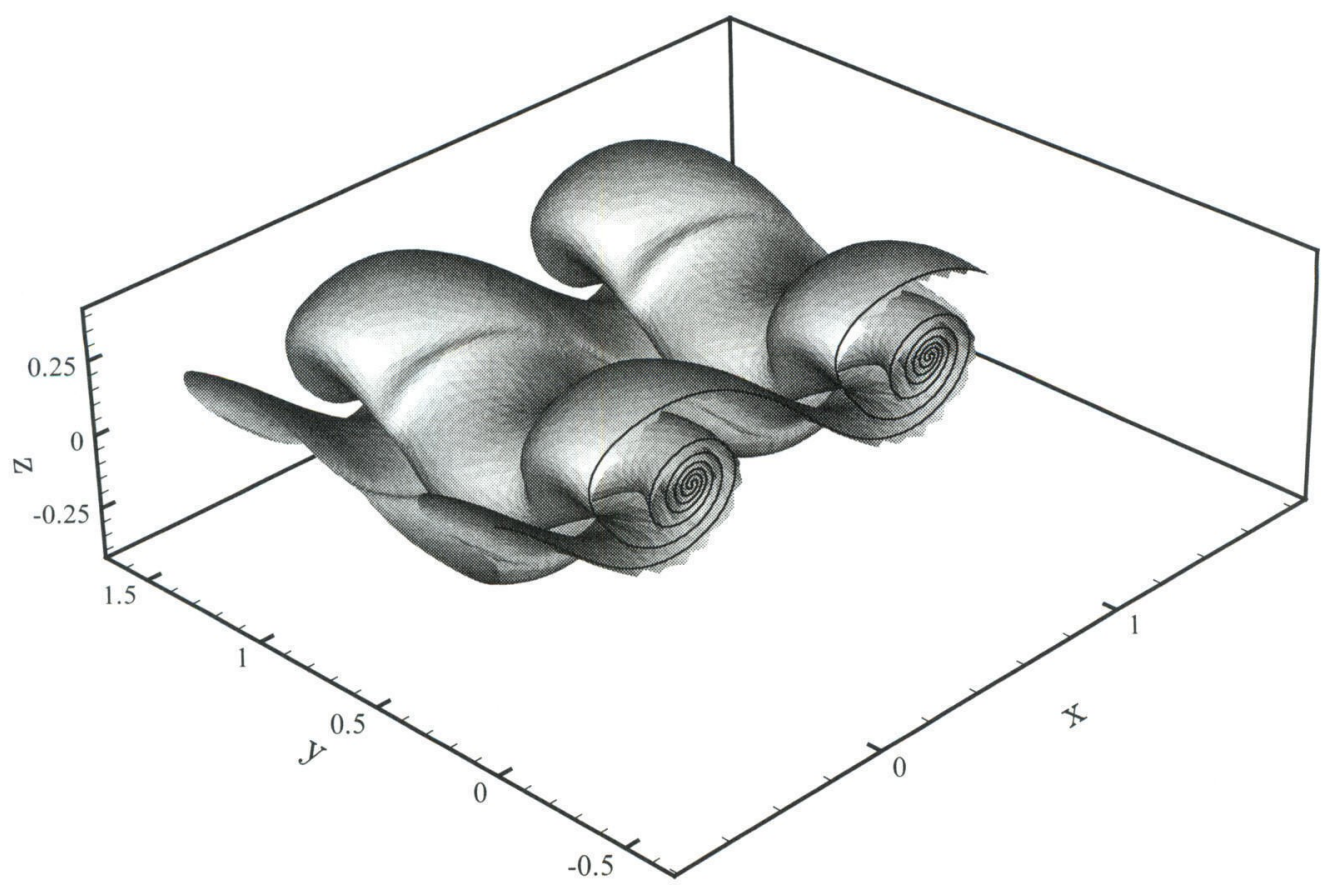

(d)

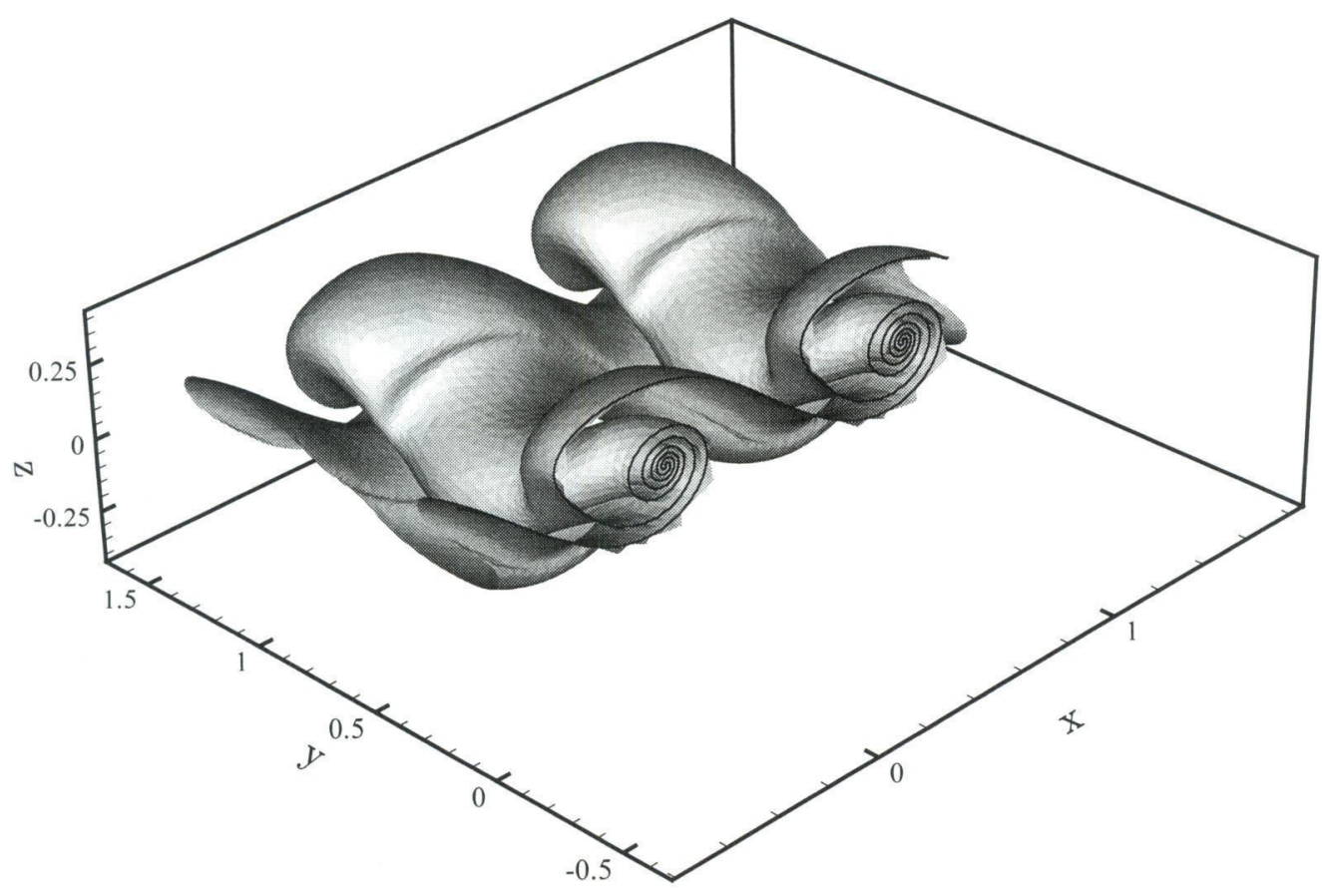

Figure 9.4: (cont) (c) $y=0.125$, and (d) $y=0.25$ reveal the deformation of the Kelvin-Helmholtz roll-up core. 
(a)

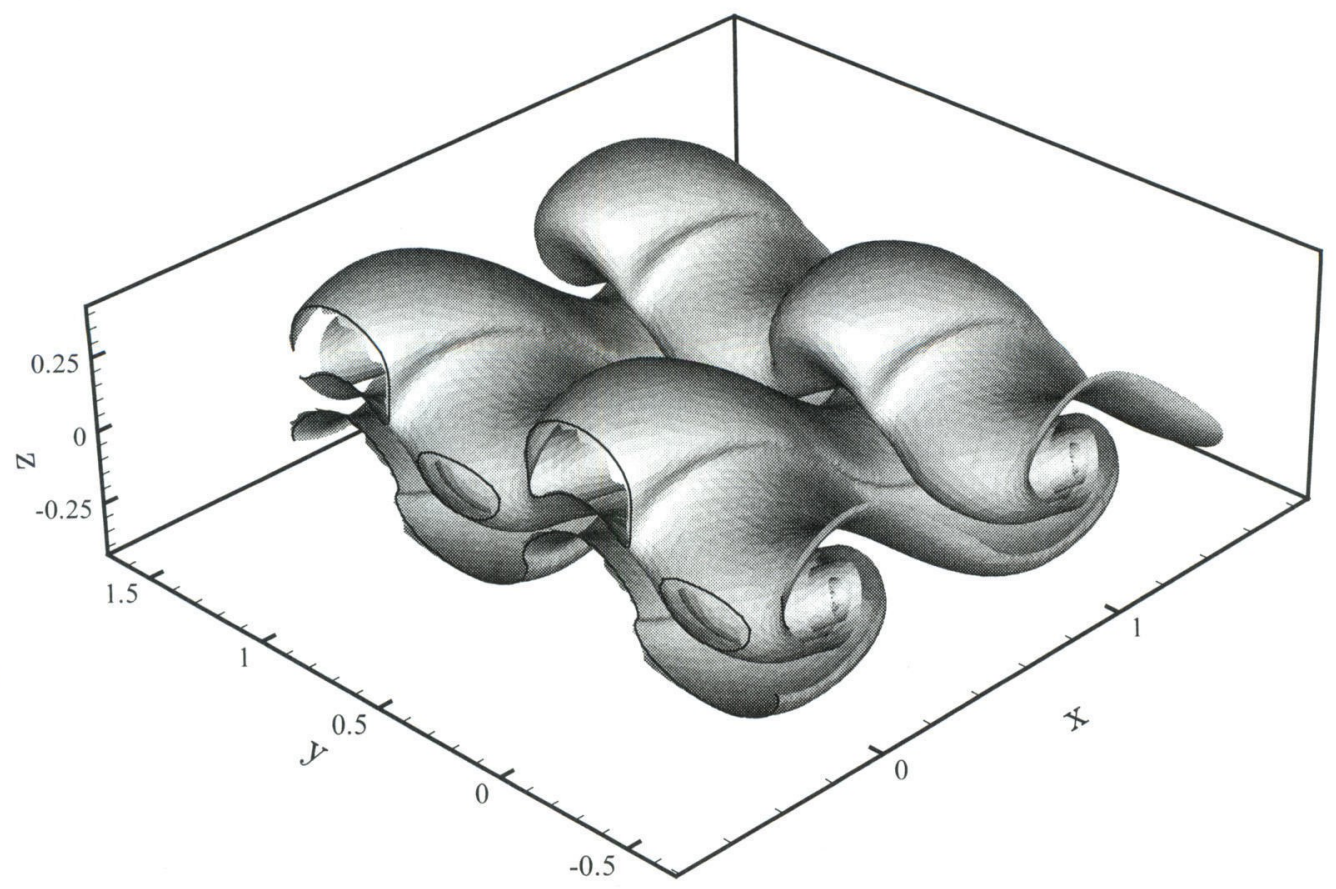

(b)

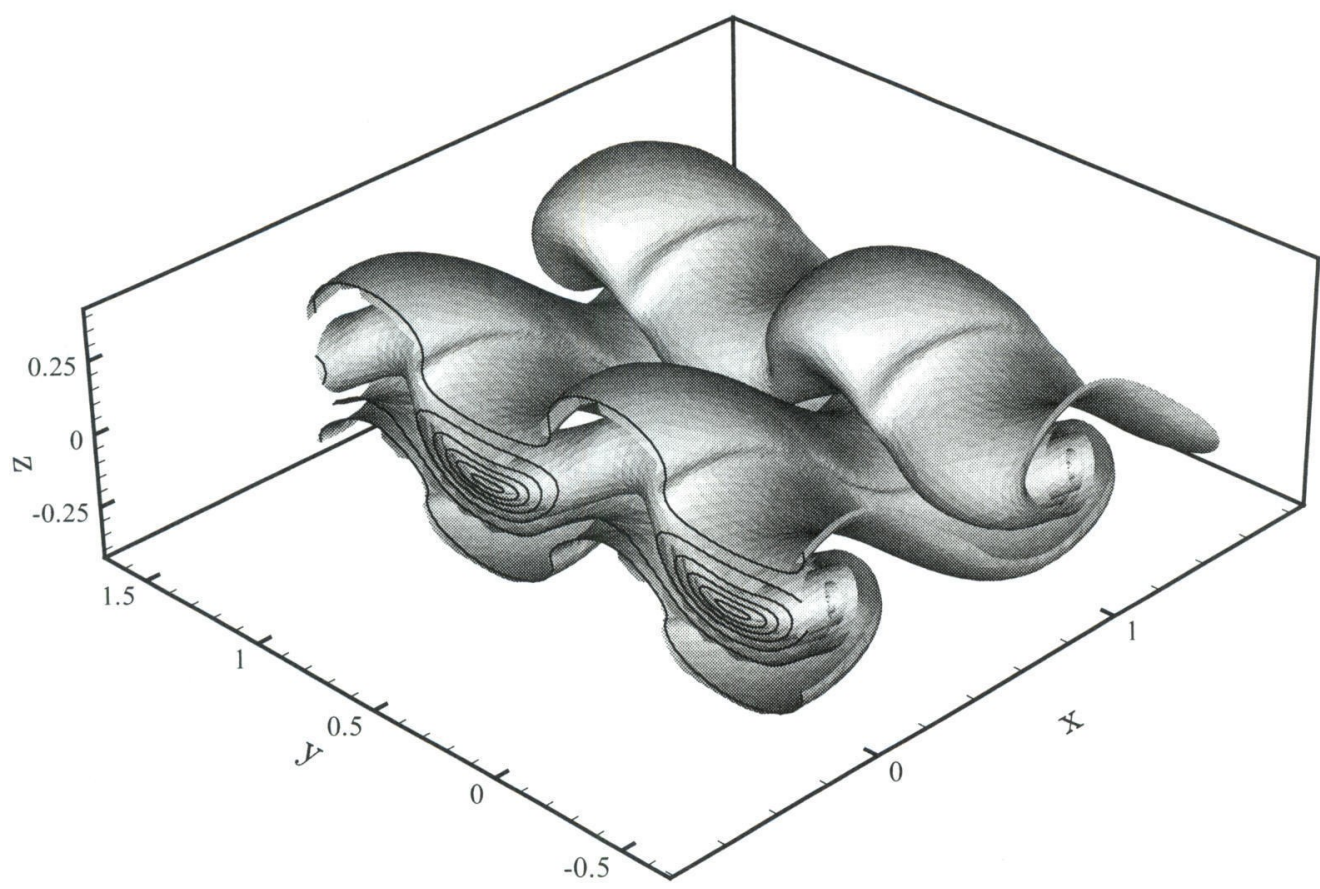

Figure 9.5: Variation of the Kelvin-Helmholtz roll-up with streamwise location at the final computed time, $t=1.8$. (a) $x=-0.25$, (b) $x=-0.125$. 
(c)

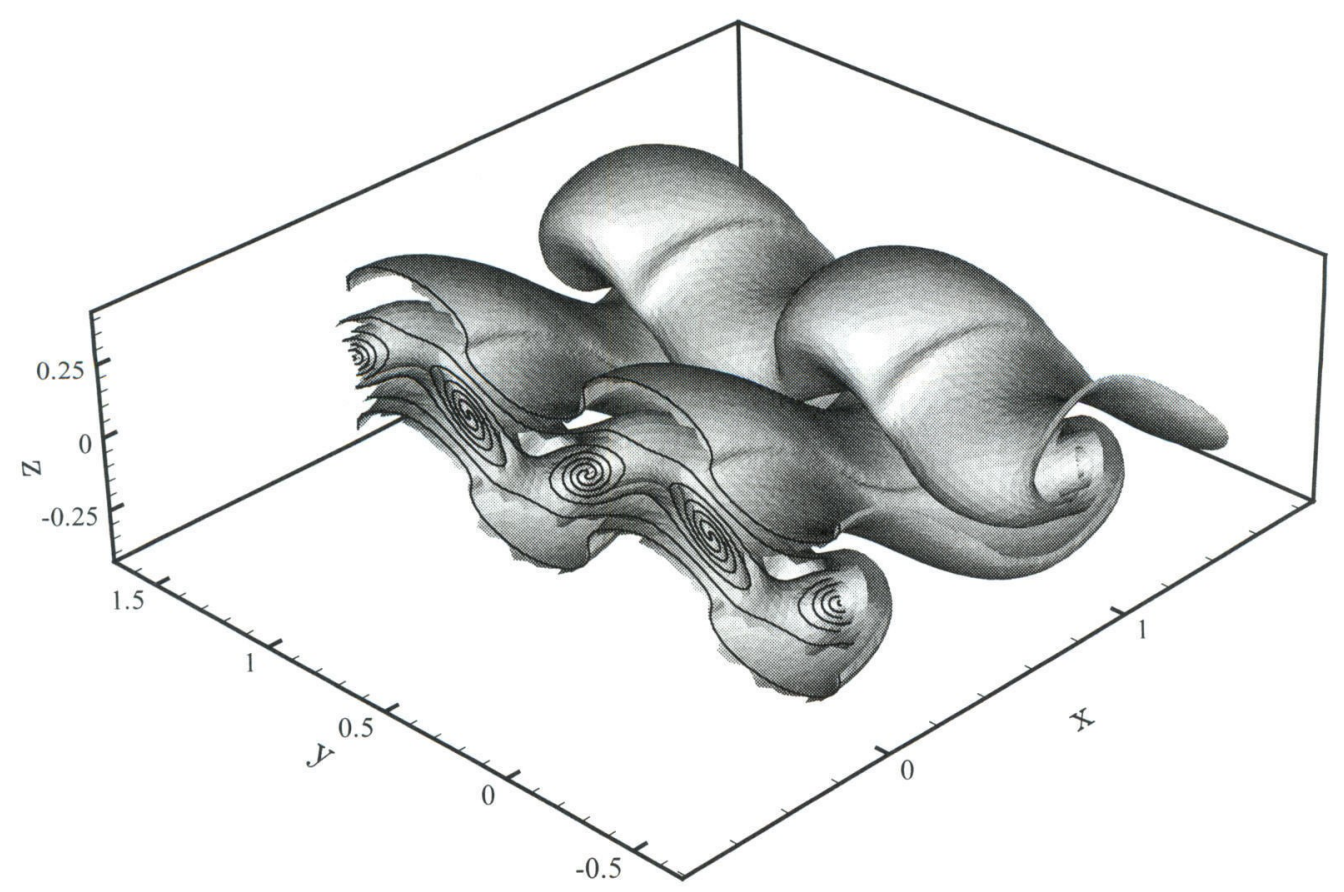

(d)

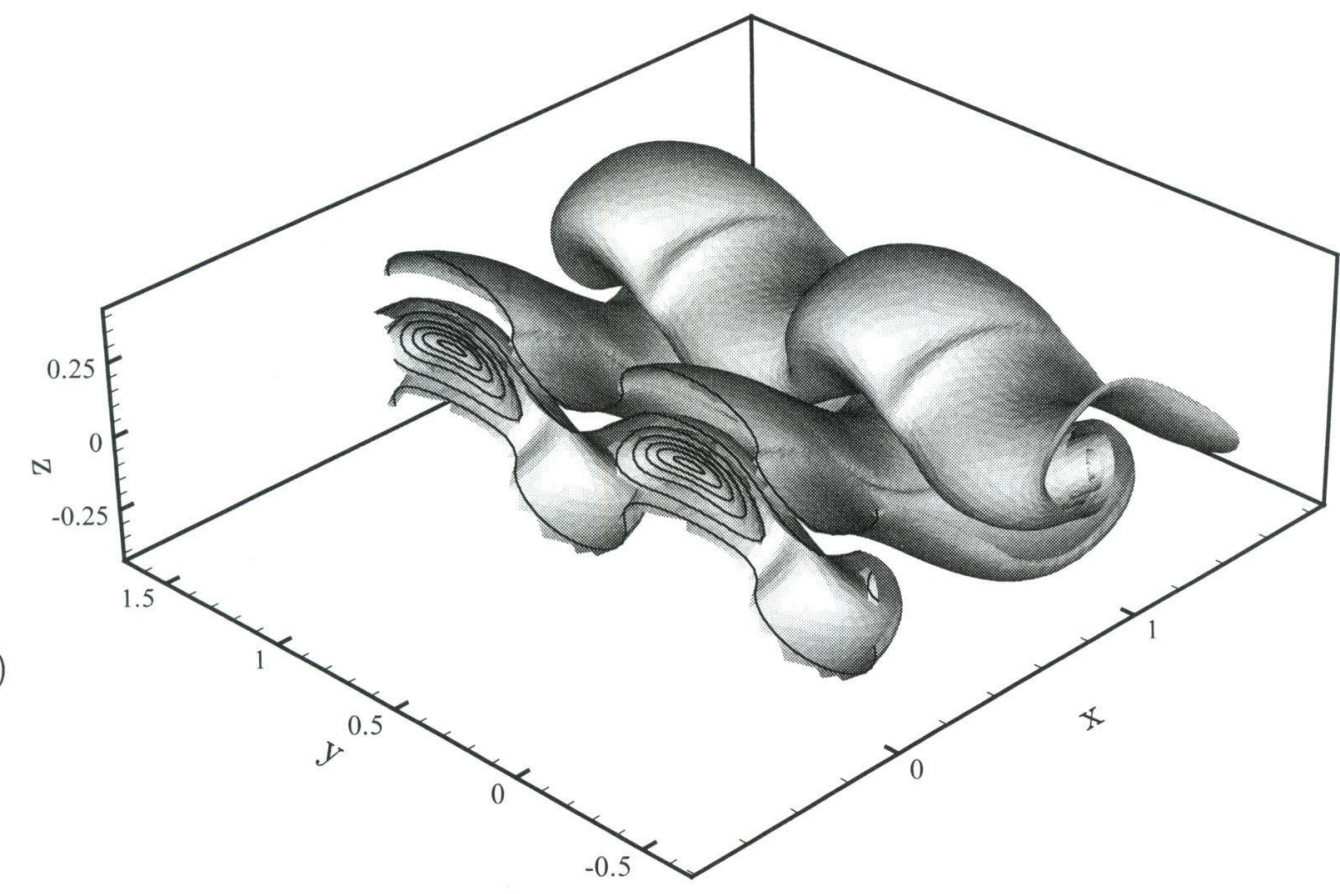

Figure 9.5: (cont) (c) $x=0$, and (d) $x=0.125$. The Kelvin-Helmholtz core perturbations are clearly visible in (c), as revealed by the roll-up of the core as is passes into and out of the cutting plane. 
(a)

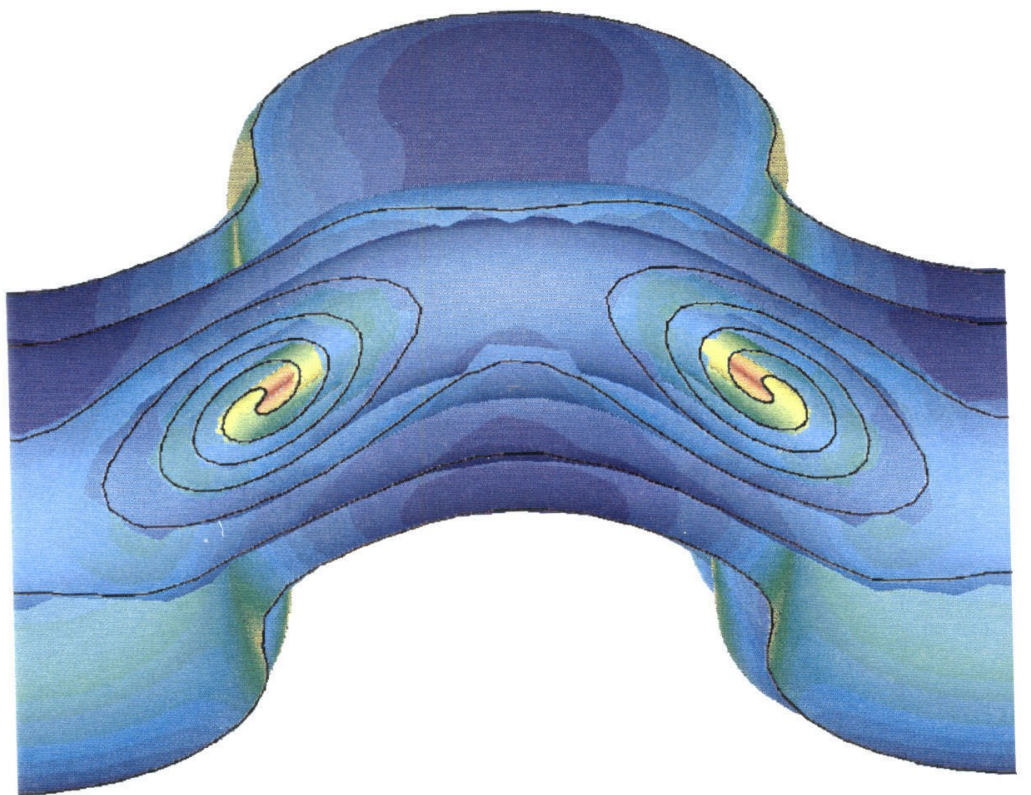

(b)

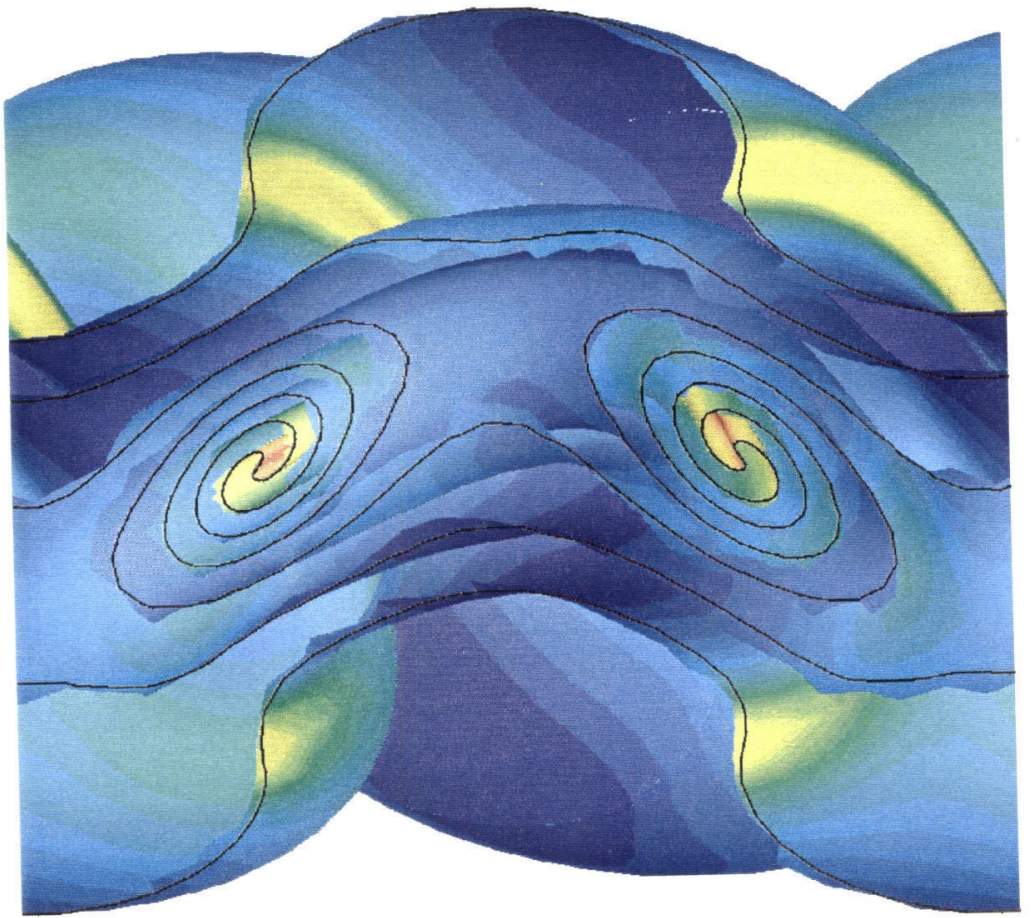

Figure 9.6: Close-up of spiral region in Fig. 9.5(c), shaded with sheet strength magnitude, $|\boldsymbol{\gamma}|$, from maximum in red to minimum in blue. Head on (a), and at a slight angle (b). 
(a)

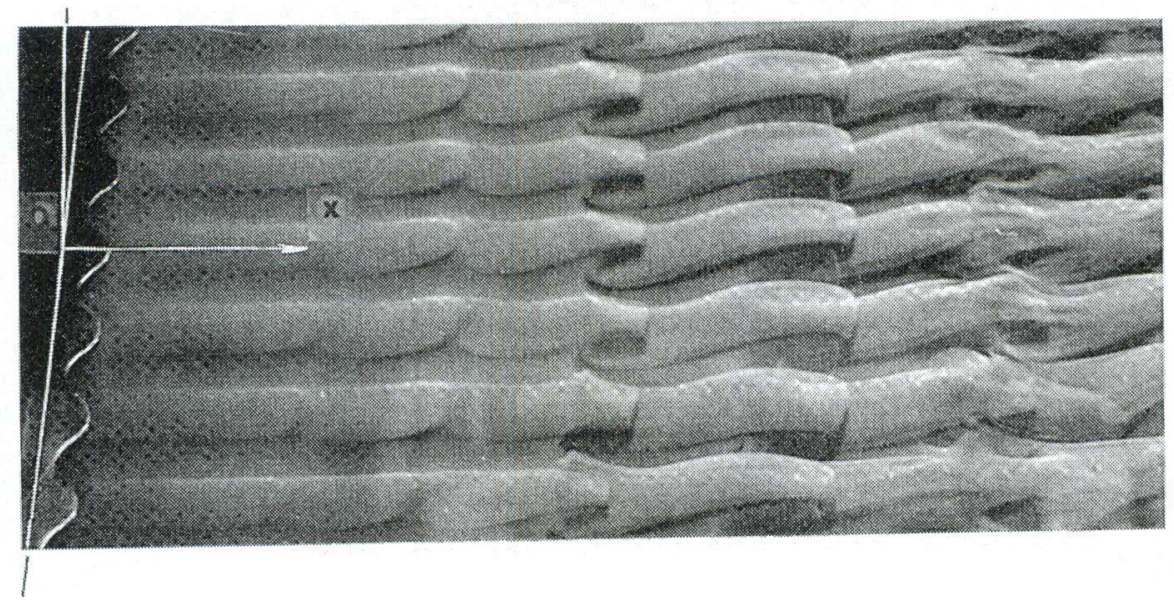

(b)

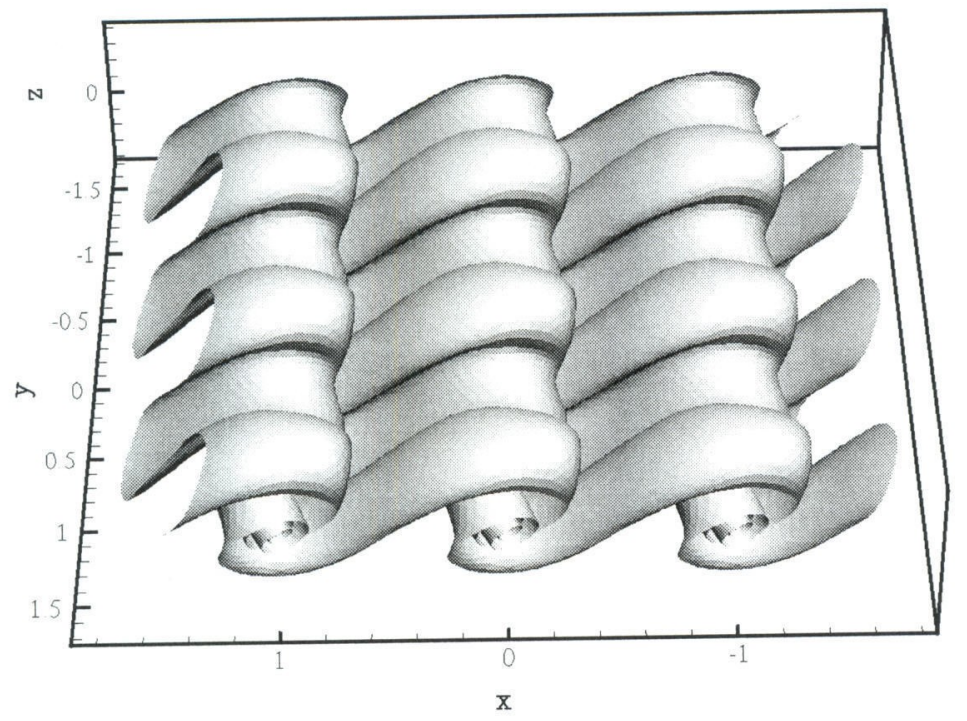

Figure 9.7: Similarity of perturbed shear layer evolution: (a) snapshot of the slowspeed side of a spatially and temporally developing shear layer (experiment by Lasheras \& Choi [30]), (b) shear layer interface of doubly periodic calculation as described in $§ 9.1$. See text for conditions of the experiment. 


\section{Chapter 10 Vortex Sheet Critical Time}

We now wish to focus on a different aspect of vortex sheet roll-up. Rather than examining long-time behavior with a fixed smoothing parameter $\sigma>0$, as in the previous two chapters, we now look at the $\sigma \rightarrow 0$ limit and in particular discuss a technique for predicting the singularity time of true vortex sheet motion, as mentioned in Chapter 1. We apply this technique on the doubly periodic planar shear layer, as the $2 \mathrm{D}$ simplification of this configuration has been studied extensively.

\subsection{Background}

The non-linear evolution of a doubly infinite, uniform vortex sheet subject to small out-of-plane disturbances is a classic problem in fluid mechanics. It has long been known that the linearized Kelvin-Helmholtz instability of the vortex sheet subject to two-dimensional shape disturbances results in an ill-posed initial-value problem [8,52]

- see Saffman [51] for a discussion. Moore [38] studied the non-linear problem by constructing approximate solutions of the exact Birkhoff-Rott equation for the vortex sheet evolution, demonstrating the formation of a finite-time singularity in the form of an infinite sheet curvature and a cusp in the sheet strength (local velocity jump), in finite time. The problem may seem academic but does have practical relevance to the point-wise convergence of numerical solutions to the compressible Euler equations, where slip surfaces (vortex sheets) are produced at triple points [54]. For convective Mach numbers (difference in velocity over the sum of the sound speeds) less than than unity, these vortex sheets in compressible Euler flow are also ill-posed [36]; and hence it is difficult for calculations in this regime to converge to an appropriate weak solution for times greater than the singularity time as the mesh size, and hence artificial viscosity is reduced.

In this chapter we present a new technique for estimating the time of singularity 
formation $\left(t_{c}\right)$ of vortex sheets evolving in an incompressible, inviscid flow. The method is particularly useful in this thesis, as it can be used with regularized vortex sheet calculations. Though general in nature, we apply the method to shear layer simulations, as this geometry has been studied extensively in $2 \mathrm{D}$. We begin with a review of Moore's [38] analytic result for $2 \mathrm{D}$ vortex sheets and the subsequent implementation by Krasny [28] for 2D normal mode perturbations.

\subsection{Finite-time Singularity for 2D Vortex Sheets}

We define a strictly $2 \mathrm{D}$ vortex sheet evolution by

$$
\mathbf{X}(\xi, \eta, t)=\left(\begin{array}{c}
g_{1}(\xi, t) \\
\eta \\
g_{2}(\xi, t)
\end{array}\right), \quad \Gamma=g_{3}(\xi), \quad \mathbf{W}=\frac{\partial g_{3}}{\partial \xi}\left(\begin{array}{l}
0 \\
1 \\
0
\end{array}\right)
$$

The field velocity (from Eq. 2.22) is then

$$
\mathbf{u}(\boldsymbol{x})_{2 D}=\frac{1}{2 \pi} \int_{-\infty}^{\infty}\left(\frac{\partial g_{3}}{\partial \xi}\right) \frac{1}{\left(x-g_{1}\right)^{2}+\left(z-g_{2}\right)^{2}}\left(\begin{array}{c}
z-g_{2} \\
0 \\
-x+g_{1}
\end{array}\right) d \xi
$$

It follows from Eq. 10.1 and Eq. 10.2 that the vortex sheet shape is invariant in the $y$-direction and that vortex lines remain parallel to the $y$-axis. When $x \rightarrow \mathbf{X}$ on the sheet and the left-side of Eq. 10.2 is replaced by $\partial \mathrm{X} / \partial t$, a version of the BirkhoffRott equation for $2 \mathrm{D}$ vortex sheet evolution results, the integrals of which are of the Cauchy Principal Value type.

Moore [38] showed that the 2D evolution of a vortex sheet (see Fig. 10.1 for a sketch of the shear layer model) from initial conditions given, in our notation, by

$$
g_{1}(\xi)=\xi, \quad g_{2}(\xi)=\epsilon \sin k \xi, \quad g_{3}(\xi)=-U \xi
$$

where $k=2 \pi / \lambda, \lambda$ is the wavelength and $\epsilon$ is the initial disturbance height, results 


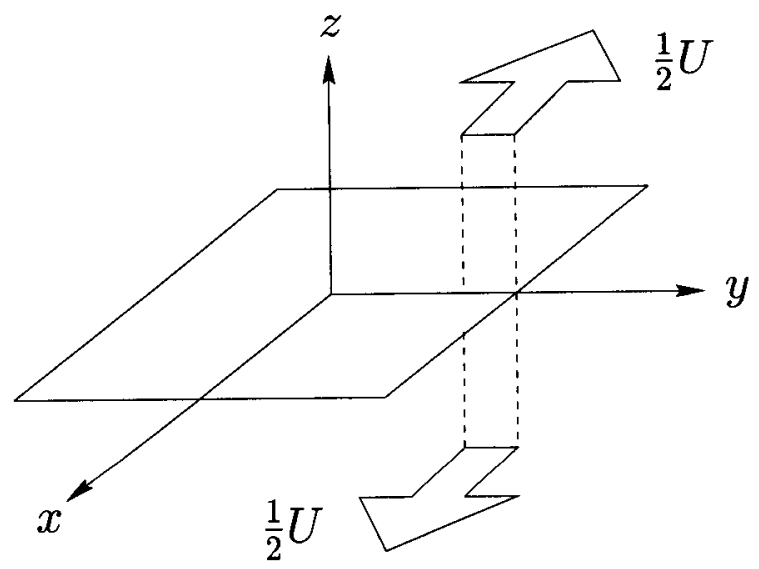

Figure 10.1: Sketch of a planar vortex sheet with velocity jump $U$.

in a curvature singularity in the sheet shape at a time $t_{c}$ given by

$$
1+\pi \tilde{t}_{c}+\ln \left(2 \pi \tilde{t}_{c}\right)=\ln \left(\frac{2}{\pi \tilde{\epsilon}}\right)
$$

where $\tilde{\epsilon}=\epsilon / \lambda$ and $\tilde{t}_{c}=t_{c} U / \lambda$. The mechanism of singularity formation is the focusing of vorticity onto the singularity point by the non-linear sheet dynamics for $t>0$. This particular choice of initial disturbance does not correspond to a normal mode of the linearized Birkhoff-Rott evolution equation; however, a modification of Moore's result for a normal mode disturbance was given by Krasny [28], who showed that the initial sheet disturbance of wavelength $\lambda$ given by

$$
g_{1}(\xi)=\xi-\epsilon \sin (k \xi), \quad g_{2}(\xi)=\epsilon \sin (k \xi), \quad g_{3}(\xi)=-U \xi
$$

produced Moore's curvature singularity at a time $t_{c}$ given by

$$
1+\pi \tilde{t}_{c}+\ln \left(\pi \tilde{t}_{c}\right)=\ln \left(\frac{1}{2 \pi \tilde{\epsilon}}\right)
$$

\subsection{Numerical Estimation of the Critical Time, $t_{c}$}

Previous numerical studies of the critical time have involved calculation of true (un-smoothed) vortex sheet motion, primarily in 2D [28]. Presented here is a method 
which generalizes easily to $3 \mathrm{D}$ and is suitable to implement with calculations of regularized vortex sheet motion.

The maximum curvature of the sheet $\kappa_{\mathrm{m}}$ is assumed to behave, near the critical time, as

$$
\kappa_{\mathrm{m}}=\frac{\beta}{\left(t_{c, \sigma}-t\right)^{\alpha}}
$$

where $\alpha, \beta$ and $t_{c, \sigma}$ are unknown constants depending on the initial condition and the regularization parameter, $\sigma$. The location in the sheet where the curvature obtains its maximum value shifts slightly with time; however, near the critical time, we find in all cases that this location converges to a specific material point on the sheet. The critical time is determined by examining the run-generated data $\left(t, \kappa_{\mathrm{m}}\right)$ to find the best linear fit (in terms of maximizing the correlation coefficient) to

$$
\kappa_{\mathrm{m}}^{-1 / \alpha}=\beta^{-1 / \alpha}\left(t_{c, \sigma}-t\right)
$$

for all feasible values of $(\alpha, \beta)$ and a specified time range $t \in\left[t_{1}, t_{2}\right]$. We define $t_{2}$ as the time at which the $\left(t, \kappa_{\mathrm{m}}\right)$ curve first changes concavity. Then $t_{1}$ is set as a high percentage of $t_{2}$, e.g., $t_{1}=0.85 t_{2}$ (see Fig. 10.2). As shown in the next section, with smooth data, the technique is fairly insensitive to the percentage chosen.

Thus far, the critical time $t_{c, \sigma}$ is a function of the initial condition, evolution equations, and smoothing parameter $\sigma$ (assuming an accurate integrated solution is obtained). The quantity of interest, of course, is the limiting value of $t_{c, \sigma}$ as $\sigma \rightarrow 0$ for a given initial condition. This value is obtained via extrapolation of the $\left(\sigma, t_{c, \sigma}\right)$ data to $\sigma=0$ with a quadratic fit, as will be shown in Fig. 10.4. 


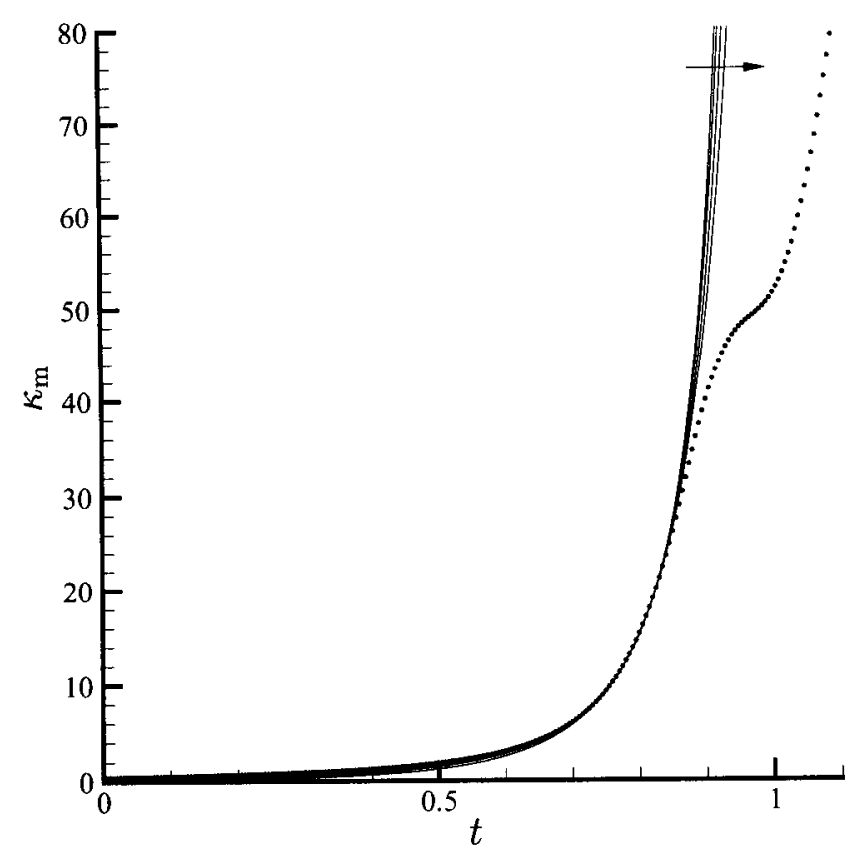

Figure 10.2: Example $\left(t, \kappa_{\mathrm{m}}\right)$ data (dots) from 2D evolution defined by Eq. D.3 and sheet parameters $\epsilon=0.01, \sigma=.05$ (see Eq. 10.9). Also four different curve fits (lines from left to right following arrow) of Eq. 10.8 with $t_{1}=0.75 t_{2}, 0.8 t_{2}, 0.85 t_{2}$ and $0.9 t_{2}$. In this case $t_{2}=0.84$.

\subsubsection{D evolution, 2D initial condition}

Consider the initial condition (periodic in $\xi$ with unit period)

$$
\begin{aligned}
X(\xi, 0) & =\xi+\epsilon \sin 2 \pi \xi \\
Y(\xi, 0) & =-\epsilon \sin 2 \pi \xi \\
\Gamma(\xi) & =\xi
\end{aligned}
$$

which is the one considered in [28]. Since this is a purely 2D initial condition, Eq. 2.22 simplifies to the regularized, periodic Birkhoff-Rott equation (see Appendix D). As detailed in the appendix, it is interesting to note that the order of adding regularization and periodicity to the Birkhoff-Rott kernel does have a minor effect on the form of the final $2 \mathrm{D}$ evolution equations, though their $\sigma \rightarrow 0$ limit is, of course, the 
same. An example of the sheet evolution (subject to Eq. D.3) is shown in Fig. 10.3. Several calculations are performed, with both $2 \mathrm{D}$ evolution equations and several values of the regularization parameter $\sigma$ and perturbation amplitude $\epsilon$. All calculations in this section are done with the number of vortex blobs fixed at 500, trapezoidal spatial integration, and RK4 temporal integration with $\Delta t=0.005$. As one would assume necessary, we find the extrapolated $(\sigma \rightarrow 0)$ values of $t_{c}$ to be independent of the evolution equations chosen (between Eq. D.2 or Eq. D.3), as demonstrated in Fig. 10.4. Furthermore, we are able to reproduce the numerical critical time findings of [28] with acceptable accuracy using this technique, as shown in Fig. 10.5, for several slightly different initial conditions (variations in the perturbation magnitude $\epsilon$ ). With singularity prediction success in a purely $2 \mathrm{D}$ initial condition and evolution, we move to calculations with the full $3 \mathrm{D}$ evolution equations.

\subsubsection{D evolution, 2D initial condition}

We now present an extension of the calculations done in the previous section. Equivalent initial conditions, extruded into a 3D sheet, are used with the full $3 \mathrm{D}$ evolution equations (Eq. 2.22). Specifically, we have

$$
\mathbf{X}(\xi, \eta, t=0)=\left(\begin{array}{c}
\xi / 2 \pi+\epsilon \sin \xi \\
\eta / 2 \pi \\
-\epsilon \sin \xi
\end{array}\right) \quad \Gamma(\xi, \eta)=-\xi / 2 \pi
$$

An example initial condition and subsequent evolution is shown in Fig. 10.6.

The same critical time estimation technique is used again, but instead of finding the maximum curvature $\left(\kappa_{\mathrm{m}}\right)$ over the entire sheet surface, independent spanwise slices of the sheet are considered instead. In this manner we calculate the critical time as a function of the spanwise location $y_{s}$. The maximum curvature of each slice is taken as the maximum principal curvature $\left(\kappa_{a}\right)$ on the slice. Again calculations were done, for several values of $\sigma$ and fixed initial amplitude $\epsilon=0.01$. Fig. 10.7 shows the extrapolated critical time as a function of the spanwise position $y_{s}$ (for the selected 

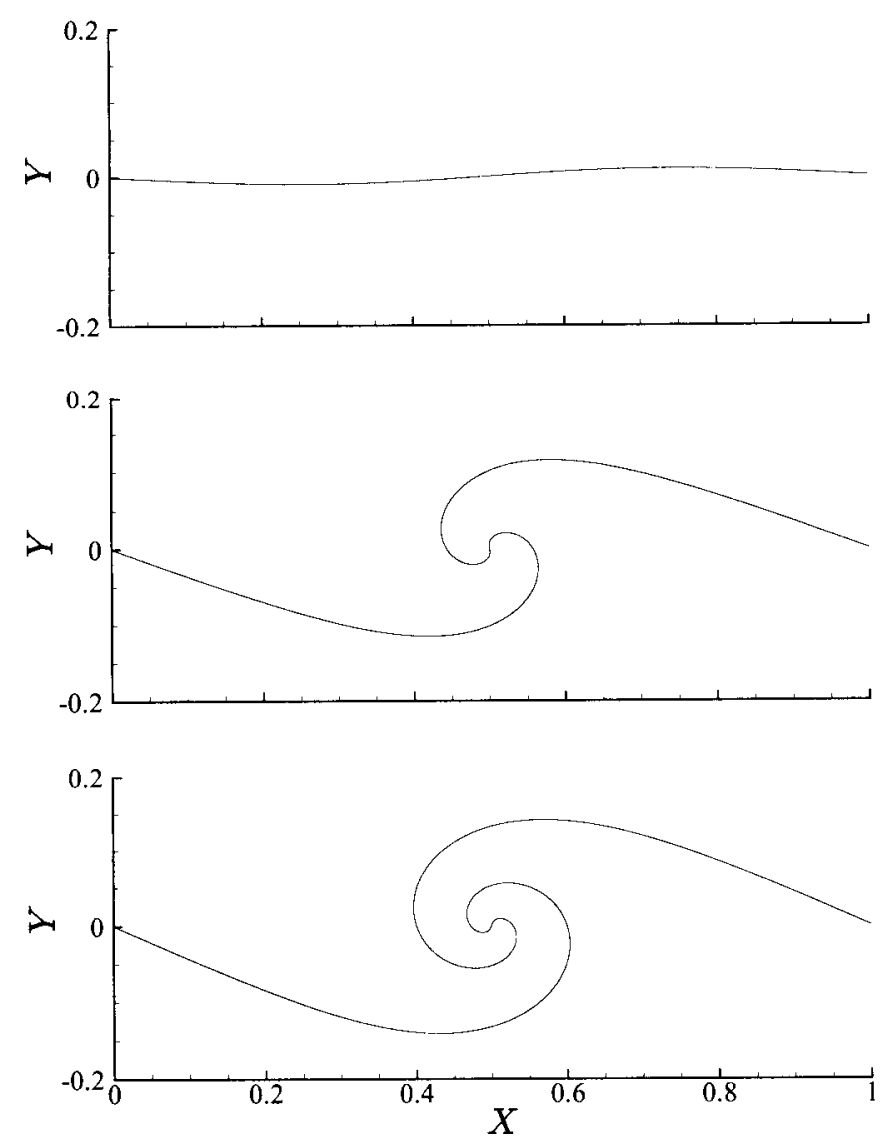

Figure 10.3: Example roll-up of a 2D, periodic vortex sheet $(\epsilon=.01$ and $\sigma=.05)$ modeling a shear layer, subject to evolution Eq. D.3. Three snapshots represent the times (a) $t=0$, (b) $t=t_{c, \sigma}$, and (c) $t>t_{c, \sigma}$.

$\epsilon=0.0375)$. As expected, $t_{c}$ remains constant due to the purely $2 \mathrm{D}$ perturbation and a benchmark is established for which to judge spanwise perturbations of $t_{c}$ for arbitrary, fully 3D initial conditions.

\subsubsection{D evolution, 3D initial condition}

Finally we consider a fully 3D initial condition: a pure $3 \mathrm{D}$ normal mode perturbation. This is a straightforward extension of the previous section, representing the most basic 3D perturbation as a solution of the linearized problem with potential differences. The 3D normal modes are detailed in the following chapter. Specifically, 


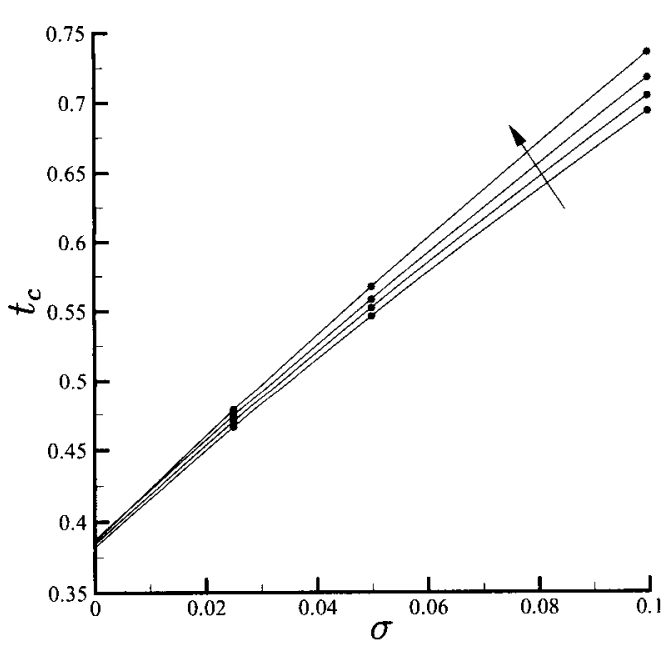

(a)

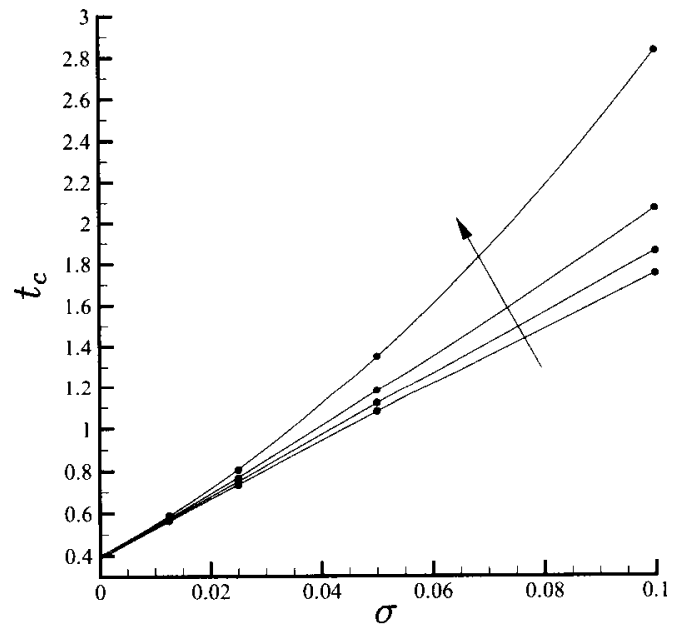

(b)

Figure 10.4: Quadratic fits used to extrapolate $t_{c, \sigma}$ to $\sigma=0$ with data from sheets driven by (a) Eq. D.2 and (b) Eq. D.3, both with initial condition given by Eq. 10.9 and $\epsilon=0.01$. Both approach $t_{c} \approx 0.39$ as $\sigma \rightarrow 0$, independent of the temporal window used ( $t_{1}$ is a larger fraction of $t_{2}$ following arrow).

we take

$$
\mathbf{X}(\xi, \eta, t=0)=\left(\begin{array}{c}
\xi / 2 \pi \\
\eta / 2 \pi \\
\epsilon \cos (\xi+\eta)
\end{array}\right) \quad \Gamma(\xi, \eta)=-\xi / 2 \pi-\frac{\epsilon}{\sqrt{2}} \cos (\xi+\eta)
$$

as depicted in Fig. 10.8(a). The geometric perturbation is only 2D (cylindrical) when viewed along the appropriate axis, as shown in Fig. 10.8(b), but the vorticity distribution is not $2 \mathrm{D}$ in any sense.

When calculating the motion of these regularized vortex sheets at various amplitudes $\epsilon$ and with different values of $\sigma$, we noticed the sheet geometry remained $2 \mathrm{D}$ when viewed along the appropriate axis. This surprising observation lead us to consider the motion of true vortex sheets, evolving from this normal mode initial condition, from an analytic standpoint rather than simulation and follows in the next chapter. 


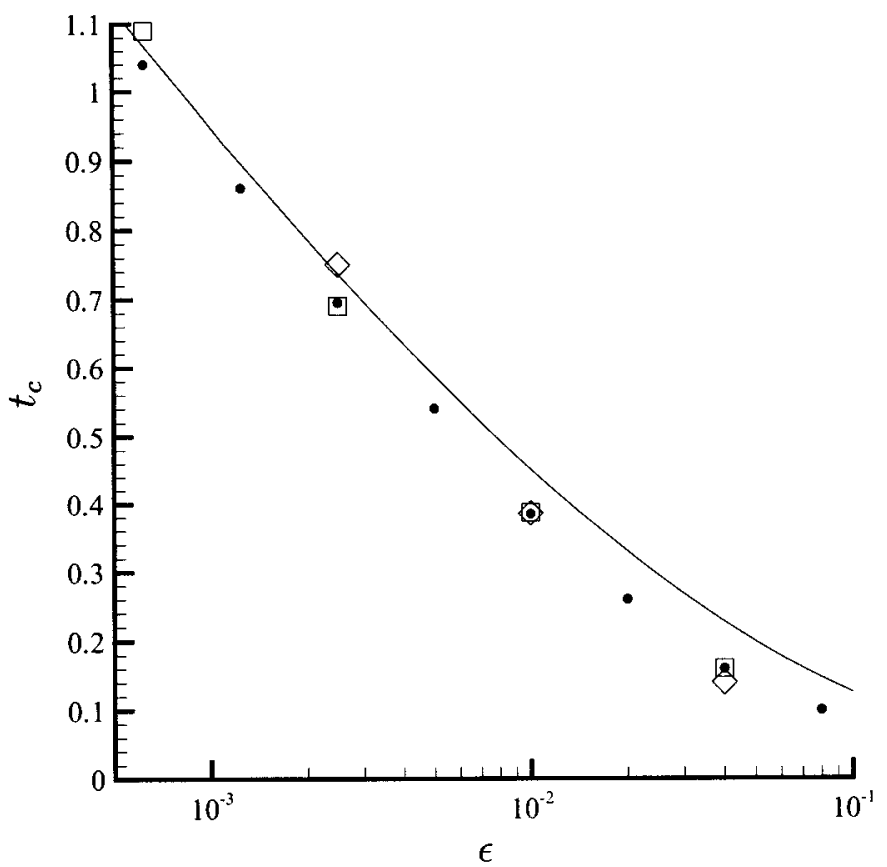

Figure 10.5: Good agreement with techniques used to evaluate $t_{c}$ : data from [28] $(\bullet)$; extrapolated $t_{c}$ values from Eq. D.2 $(\square)$; extrapolated $t_{c}$ values from Eq. D.3 $(\diamond)$; theoretical prediction from Eq. 10.6 (line). 


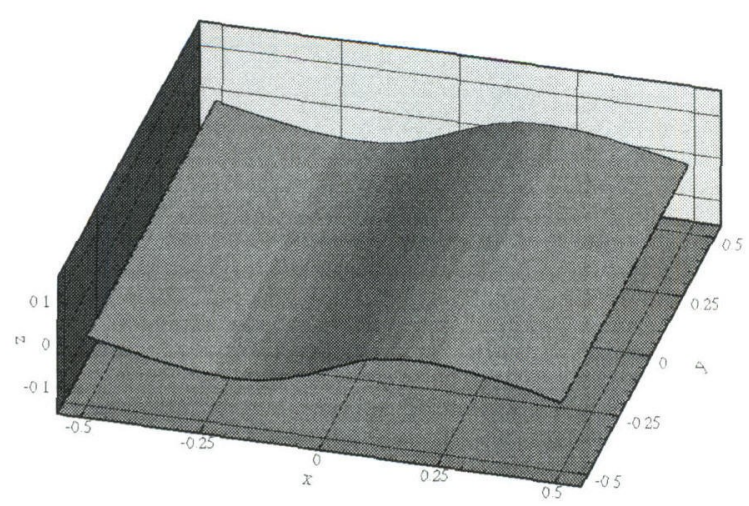

(a)

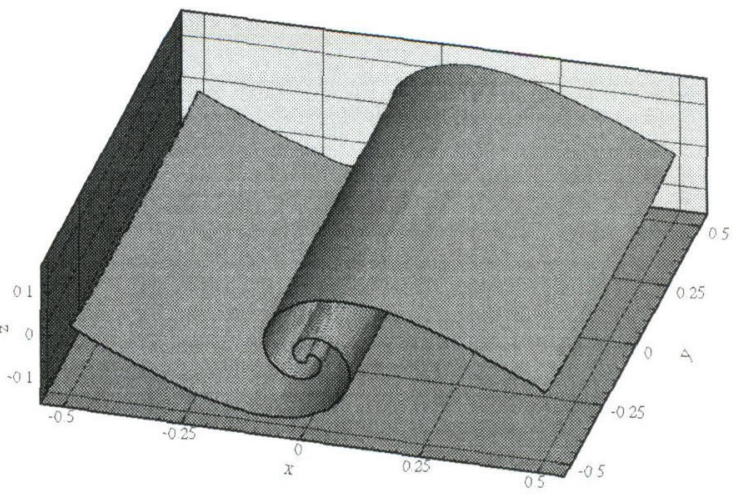

(b)

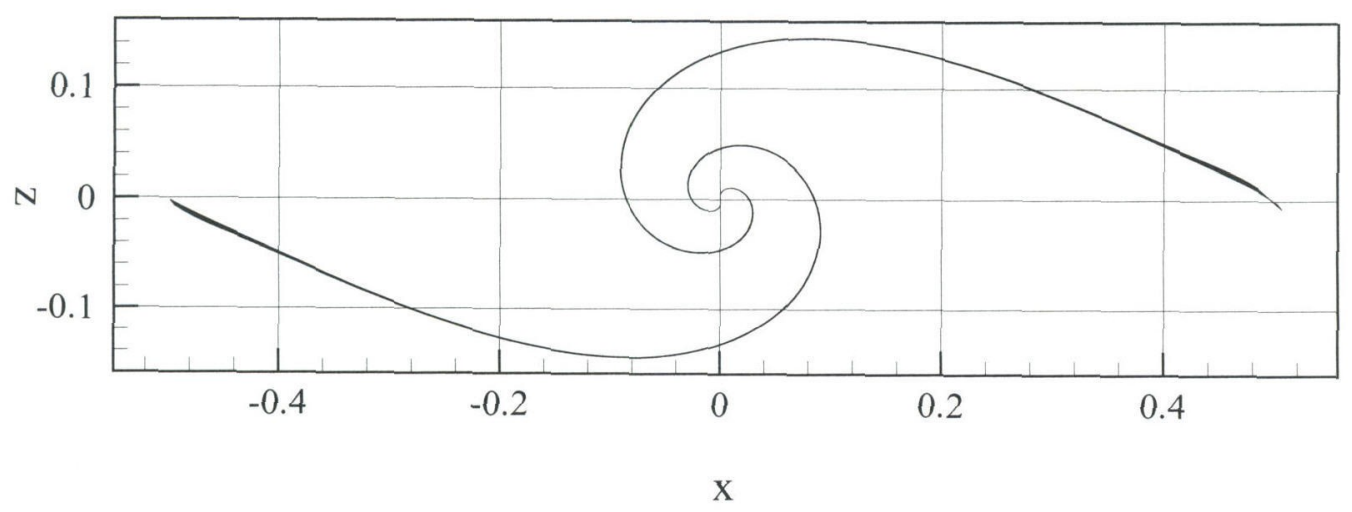

(c)

Figure 10.6: One period ( $P_{0}$ from Fig. 6.1$)$ of the doubly periodic shear layer vortex sheet (Eq. 10.10) with $\sigma=0.0375, \epsilon=0.05$, and (a) $t=0$, (b) $t>t_{c, \sigma}$. Even for large time $t$, the solution remains $2 \mathrm{D}$, as shown by the projected side view (c). 


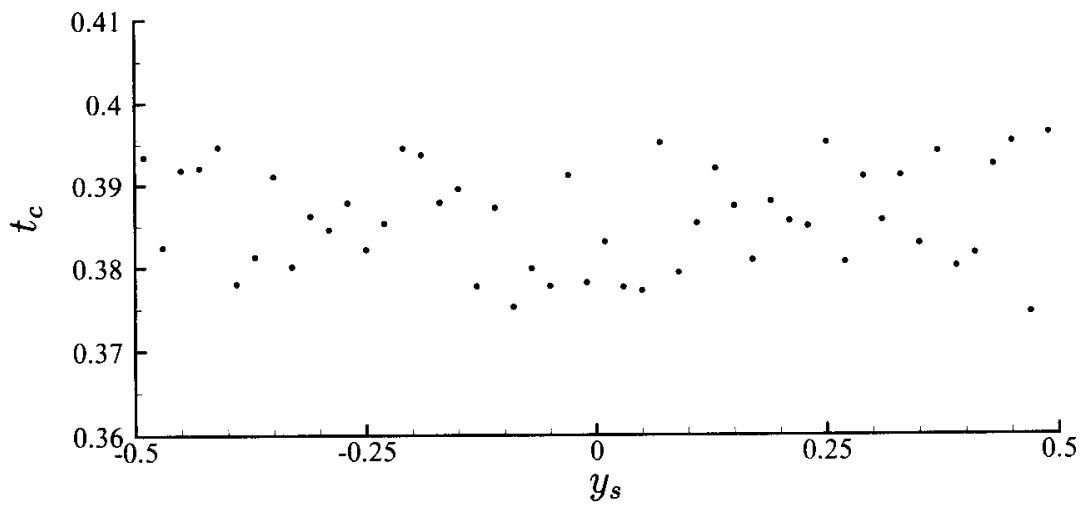

Figure 10.7: Extrapolated $(\sigma \rightarrow 0)$ singularity time for the 3D calculation of a $2 \mathrm{D}$ initial condition, as a function of spanwise position, $y_{s}$. Compare with corresponding $\epsilon=0.01$ in Fig. 10.5 . 
(a)

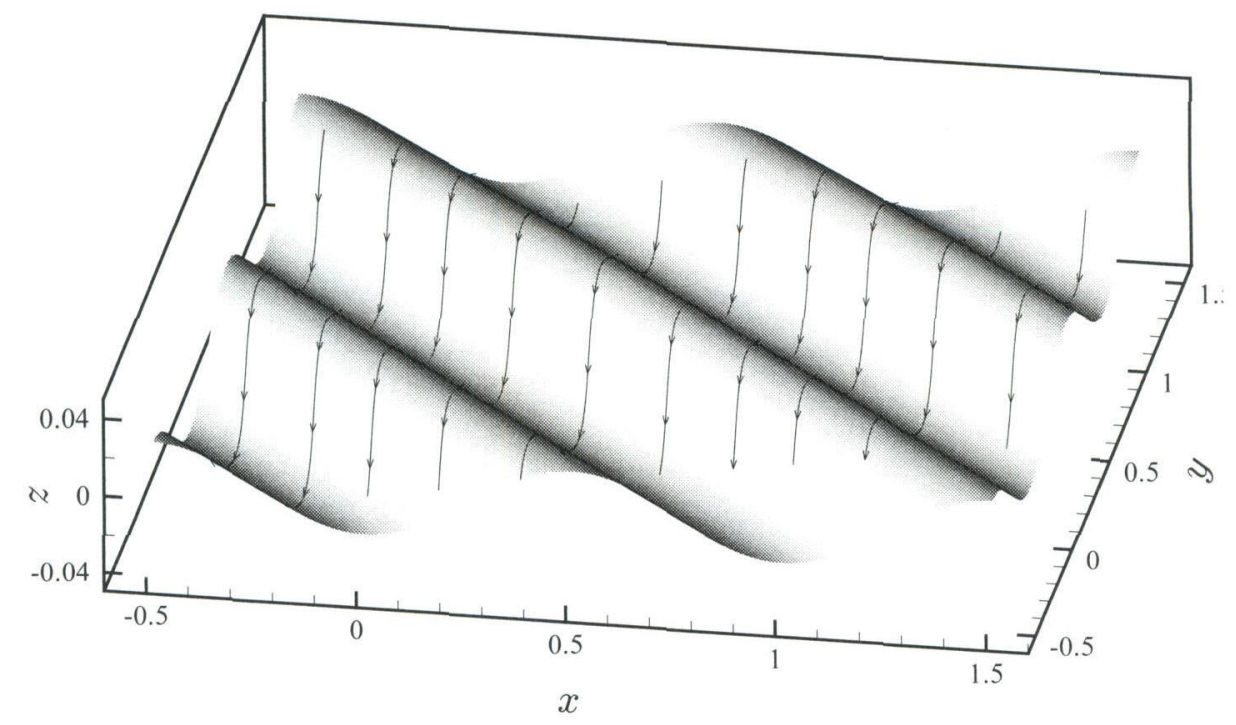

(b)

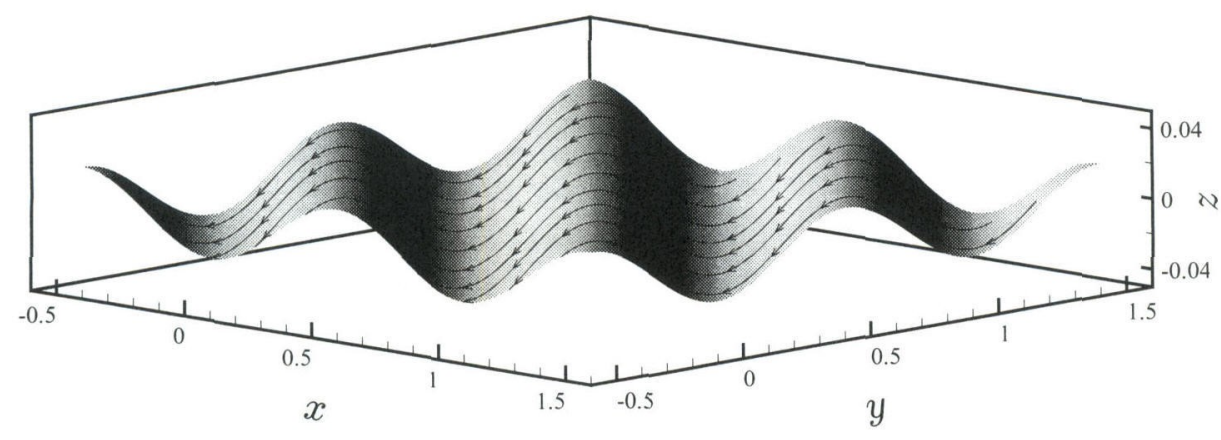

Figure 10.8: 3D normal mode initial condition and associated vortex lines. Flow is in the positive $x$ direction below the sheet, negative $x$ direction above the sheet. Vertical scale is exaggerated to demonstrate geometry: (a) view from above; (b) rotated view from above, revealing 2D geometry. Vortex lines are not exactly parallel to the $y$ axis. 


\section{Chapter 11 3D Normal Mode Perturbations}

\subsection{Introduction}

In this chapter we consider the true vortex sheet evolution when subject to a disturbance which is a finite amplitude 3D normal mode of the linear stability problem. As such we return to the non-smoothed $(\sigma=0)$ Biot-Savart kernel and the hazards ill-posedness and singularity formation as described in Chapter 1. In fact, the goal of the analysis in this chapter is to characterize the singularity formation of the $3 \mathrm{D}$ vortex sheet in terms derived from what is already known about $2 \mathrm{D}$ singularity formation. The analyzed 3D normal mode is cylindrical, in a defined sense. It is shown that the full non-linear sheet motion then remains cylindrical and that it is identical to a suitably chosen, strictly $2 \mathrm{D}$ vortex sheet evolution. It follows that the singularity formation process is the same as for a 2D normal mode disturbance, allowing a straightforward extension of Moore's result to the case of an initial disturbance consisting of a finite amplitude, 3D normal mode.

\subsection{Vortex Sheet Evolution}

Consider the motion of a doubly infinite vortex sheet in three dimensions. In Cartesian co-ordinates $(x, y, z)$ the undisturbed sheet shape lies in the $(x-y)$ plane with uniform $x$-velocities of $-\frac{1}{2} U$ for $z>0$ and $\frac{1}{2} U$ for $z<0$, where $U$ is the velocity jump magnitude across $z=0$ in the $x$-direction (see Fig. 11.1). For a general sheet shape we employ a parametric surface description

$$
\mathbf{X}(\xi, \eta, t)=\left(\begin{array}{c}
X(\xi, \eta, t) \\
Y(\xi, \eta, t) \\
Z(\xi, \eta, t)
\end{array}\right), \quad \Gamma=\Gamma(\xi, \eta)
$$


where $(\xi, \eta)$ are Lagrangian parameters defining the sheet position $\mathbf{X}$ and the velocity potential jump $\Gamma$. Unless otherwise specified the dependence on time $t$ will be suppressed. At a field point $\boldsymbol{x}$, the velocity induced by the sheet is given by Eq. 2.19:

$$
\mathbf{u}(\boldsymbol{x})=\frac{1}{4 \pi} \int_{-\infty}^{\infty} \int_{-\infty}^{\infty} \frac{\mathbf{W} \times(\boldsymbol{x}-\mathbf{X})}{|\boldsymbol{x}-\mathbf{X}|^{3}} d \xi d \eta
$$

Recall (see §2.3) when $\boldsymbol{x} \rightarrow \mathbf{X}$ on the sheet, the left-hand side of Eq. 11.2 can be replaced by $\partial \mathbf{X} / \partial t$ resulting in a Lagrangian equation [10] for the sheet evolution from given initial conditions $\mathbf{X}(\xi, \eta, t=0), \Gamma(\xi, \eta)$. The integral must then be interpreted as a Hadamard principal-part integral; thus sheet velocities are the average of the sheet-induced velocity when $\boldsymbol{x} \rightarrow \mathbf{X}$ from either side. In the sheet evolution $\Gamma(\xi, \eta)$ is invariant with time.

\subsection{Evolution of an Initially Cylindrical Vortex Sheet}

We now introduce and define a "cylindrical" sheet. We work in Cartesian coordinates $\left(x^{\prime}, y^{\prime}, z^{\prime}\right)$ and use Lagrangian sheet co-ordinates $\left(\xi^{\prime}, \eta^{\prime}\right)$. For present purposes, a vortex sheet is said to be cylindrical at some time instant if the shape

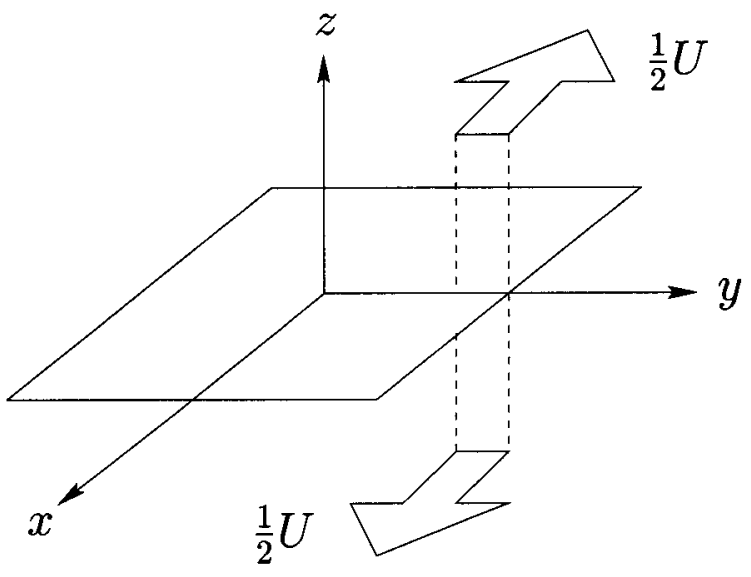

Figure 11.1: Sketch of a planar vortex sheet with velocity jump $U$. 
geometry and vorticity distribution are of the form

$$
\begin{aligned}
\mathbf{X}^{\prime}\left(\xi^{\prime}, \eta^{\prime}\right) & =\left(\begin{array}{c}
f_{1}\left(\xi^{\prime}\right) \\
\eta \\
f_{2}\left(\xi^{\prime}\right)
\end{array}\right), \quad \Gamma=f_{3}\left(\xi^{\prime}\right)+c_{1} \eta^{\prime} \\
\mathbf{W} & =\frac{\partial f_{3}}{\partial \xi^{\prime}}\left(\begin{array}{l}
0 \\
1 \\
0
\end{array}\right)-c_{1}\left(\begin{array}{c}
\partial f_{1} / \partial \xi^{\prime} \\
0 \\
\partial f_{2} / \partial \xi^{\prime}
\end{array}\right)
\end{aligned}
$$

where $c_{1}$ is a constant equal to the gradient of $\Gamma$ in the $y^{\prime}$ direction. The functions $f_{1}$ and $f_{2}$ are such that $f_{1}(\xi)=\xi+\hat{f}_{1}(\xi)$, with $\left|\hat{f}_{1}(\xi) / \xi\right| \rightarrow 0$ as $\xi \rightarrow \infty$, and $\left|f_{2}(\xi)\right|$ is bounded. A line parallel to the $y^{\prime}$-axis is the generator of the cylindrical surface. Substituting Eq. 11.3 into Eq. 11.2 gives the velocity at a general field point $\boldsymbol{x}^{\prime}$ as the sum of two components

$$
\mathbf{u}\left(\boldsymbol{x}^{\prime}\right)=\mathbf{u}\left(\boldsymbol{x}^{\prime}\right)_{2 D}+\mathbf{u}\left(\boldsymbol{x}^{\prime}\right)_{\mathbf{y}^{\prime}}
$$

where

$$
\begin{aligned}
\mathbf{u}\left(\boldsymbol{x}^{\prime}\right)_{2 D} & =\frac{1}{4 \pi} \int_{-\infty}^{\infty} \int_{-\infty}^{\infty} \frac{\partial f_{3}}{\partial \xi^{\prime}}\left(\begin{array}{l}
0 \\
1 \\
0
\end{array}\right) \times\left(\mathbf{x}^{\prime}-\mathbf{X}^{\prime}\right) \frac{d \xi^{\prime} d \eta^{\prime}}{\left|\boldsymbol{x}^{\prime}-\mathbf{X}^{\prime}\right|^{3}} \\
& =\frac{1}{2 \pi} \int_{-\infty}^{\infty}\left(\frac{\partial f_{3}}{\partial \xi^{\prime}}\right) \frac{1}{\left(x^{\prime}-f_{1}\right)^{2}+\left(z^{\prime}-f_{2}\right)^{2}}\left(\begin{array}{c}
z^{\prime}-f_{2} \\
0 \\
-x^{\prime}+f_{1}
\end{array}\right) d \xi^{\prime}
\end{aligned}
$$


and

$$
\begin{aligned}
\mathbf{u}\left(\boldsymbol{x}^{\prime}\right)_{y^{\prime}} & =-\frac{c_{1}}{4 \pi} \int_{-\infty}^{\infty} \int_{-\infty}^{\infty}\left(\begin{array}{c}
\partial f_{1} / \partial \xi^{\prime} \\
0 \\
\partial f_{2} / \partial \xi^{\prime}
\end{array}\right) \times\left(\boldsymbol{x}^{\prime}-\mathbf{X}^{\prime}\right) \frac{d \xi^{\prime} d \eta^{\prime}}{\left|\boldsymbol{x}^{\prime}-\mathbf{X}^{\prime}\right|^{3}} \\
& =\frac{c_{1}}{2 \pi} \int_{-\infty}^{\infty} \frac{\left(\partial f_{1} / \partial \xi^{\prime}\right)\left(z^{\prime}-f_{2}\right)-\left(\partial f_{2} / \partial \xi^{\prime}\right)\left(x^{\prime}-f_{1}\right)}{\left(x^{\prime}-f_{1}\right)^{2}+\left(z^{\prime}-f_{2}\right)^{2}}\left(\begin{array}{l}
0 \\
1 \\
0
\end{array}\right) d \xi^{\prime}
\end{aligned}
$$

This last integral can be evaluated analytically (straightforward as a contour integral in complex $\zeta=f_{1}+i f_{2}$, with $\left.\omega=x^{\prime}+i z^{\prime}\right)$ to give

$$
\mathbf{u}\left(\boldsymbol{x}^{\prime}\right)_{y^{\prime}}=\left(\begin{array}{c}
0 \\
\pm \frac{1}{2} c_{1} \\
0
\end{array}\right)
$$

where the plus sign holds for a field point which lies above the sheet and the minus sign applies if the field point lies below the sheet. Thus the sheet-induced velocity in the $y^{\prime}$-direction is constant and non-zero at all points above and below the sheet, when $c_{1} \neq 0$, irrespective of the sheet shape in the $\left(x^{\prime}-z^{\prime}\right)$ plane. Moreover, comparison of Eq. 11.6 and Eq. 10.2 shows that the components of the induced velocity in the $\left(x^{\prime}-z^{\prime}\right)$ are identical to an equivalent 2D sheet, in the sense described above. When $\boldsymbol{x}^{\prime} \rightarrow \mathbf{X}^{\prime}$ on the sheet, the component of the velocity in the $y^{\prime}$-direction, taken as the average of the constant values from above and below, is zero at all points on the sheet. It therefore follows that:

(i) a vortex sheet which is cylindrical at some initial time in the sense of Eq. 11.3 remains cylindrical for all time, and

(ii) the non-linear evolution of a cylindrical sheet is identical to that of an equivalent $\left(c_{1}=0\right) 2 \mathrm{D}$ vortex sheet with the same initial conditions. 


\subsection{Finite-time Singularity for 3D Vortex Sheets}

Here we obtain the shape and vorticity distributions for a 3D normal mode disturbance to the vortex sheet. The spatial parts of the normal modes obtained from a linear analysis in terms of perturbation potentials above and below the flat sheet, with uniform velocity jump $U$ in the x-direction, are

$$
\begin{aligned}
h(x, y) & =A \exp [i(m x+n y)], \\
\phi_{ \pm}(x, y) & =B_{ \pm} \exp (\mp k z) \exp [i(m x+n y)] \mp \frac{1}{2} U x,
\end{aligned}
$$

where $h$ is the disturbance in the $z$ direction, $\phi_{ \pm}$are velocity potentials above and below the sheet, $m, n$ are wave numbers in the $x, y$ directions respectively and

$$
k^{2}=m^{2}+n^{2}, \quad B_{ \pm}=\frac{1}{2} U A \frac{m}{k}(i \mp 1) .
$$

At an initial time $t=0$ this corresponds to a potential jump function

$$
\Gamma \equiv \phi_{+}-\phi_{-}=-U x-U A \frac{m}{k} \exp [i(m x+n y)]
$$

We refer to Eq. 11.10 and Eq. 11.13 as normal modes of the 3D vortex sheet.

We now rotate from $(x, y, z)$ axes to $\left(x^{\prime}, y^{\prime}, z^{\prime}\right)$ axes using

$$
k x^{\prime}=m x+n y, \quad k y^{\prime}=-n x+m y, \quad z^{\prime}=z .
$$

In $\left(x^{\prime}, y^{\prime}, z^{\prime}\right)$ axes, the sheet shape and potential jump distribution corresponding to the real parts of the linear normal mode (Eq. 11.10 - Eq. 11.13) can then be 
parameterized as

$$
\begin{aligned}
\mathbf{X}^{\prime}\left(\xi^{\prime}, \eta^{\prime}\right) & =\left(\begin{array}{c}
\xi^{\prime} \\
\eta^{\prime} \\
\epsilon \cos \left(k \xi^{\prime}\right)
\end{array}\right) \\
\Gamma & =-U\left(\frac{m}{k} \xi^{\prime}-\frac{n}{k} \eta^{\prime}\right)-U \epsilon \frac{m}{k} \cos \left(k \xi^{\prime}\right) \\
& =-U \frac{m}{k}\left[\xi^{\prime}+\epsilon \cos \left(k \xi^{\prime}\right)\right]+U \frac{n}{k} \eta^{\prime}
\end{aligned}
$$

where $\epsilon=A$. A re-parameterization $\tilde{\xi}^{\prime}=\xi^{\prime}+\epsilon \cos \left(k \xi^{\prime}\right), \tilde{\eta^{\prime}}=\eta^{\prime}$ applied to Eq. 11.15 accompanied by a phase shift, and expansion of the result to $O(\epsilon)$ gives

$$
\mathbf{X}^{\prime}\left(\xi^{\prime}, \eta^{\prime}\right)=\left(\begin{array}{c}
\xi^{\prime}-\epsilon \sin \left(k \xi^{\prime}\right) \\
\eta^{\prime} \\
\epsilon \sin \left(k \xi^{\prime}\right)
\end{array}\right), \quad \Gamma=-\frac{m}{k} U\left(\xi^{\prime}-\frac{n}{m} \eta^{\prime}\right)
$$

where following the re-parameterization we have put $\tilde{\xi}^{\prime} \rightarrow \xi^{\prime}, \tilde{\eta}^{\prime} \rightarrow \eta^{\prime}$. When $n=0$, Eq. 11.16 agrees with Eq. 10.5. When $n \neq 0$ Eq. 11.16 is the same as Eq. 10.5 if $U$ in Eq. 10.5 is replaced by $m U / k$ and the term $\eta^{\prime}$ in the $\Gamma$ equation of Eq. 11.16 is omitted. This is effectively a statement of Squires theorem for oblique disturbances. We remark that the difference in form of Eq. 11.15 and Eq. 10.5 arises because the 2D normal mode was obtained from a linear analysis of the 2D Birkhoff-Rott evolution equation [28] whilst our Eq. 11.10 is obtained from an analysis in terms of linear perturbation potentials; the expressions must agree to $O(\epsilon)$.

Comparing Eq. 11.3 to Eq. 11.16 shows that in the $\left(x^{\prime}, y^{\prime}, z^{\prime}\right)$ axes, the geometry and vorticity for the 3D normal mode correspond to our definition of a cylindrical vortex sheet with the choices

$$
f_{1}=\xi^{\prime}-\epsilon \sin \left(k \xi^{\prime}\right), \quad f_{2}=\epsilon \sin \left(k \xi^{\prime}\right), \quad f_{3}=-\frac{m}{k} U \xi^{\prime}, \quad c_{1}=\frac{n}{k} U
$$

If this is taken as a finite amplitude initial condition, then from (i) and (ii) following Eq. 11.9 we can conclude that 
(a) the evolution of the vortex sheet from an initial condition corresponding to a finite amplitude 3D normal mode disturbance remains cylindrical for all time, and

(b) in the $\left(x^{\prime}-z^{\prime}\right)$ plane its non-linear evolution is the same as that for an equivalent $2 \mathrm{D}$ normal mode with suitably modified velocity jump and ratio of disturbance amplitude to wavelength. When $n \neq 0$, the evolution proceeds with a uniform shear, with velocity difference $n U / k$, along the generator of the cylindrical sheet geometry. The vortex lines are then not parallel to the generator.

To the order of accuracy of Eq. 10.6, it follows that the evolution produces a curvature singularity at a time $t_{c}$ obtained from

$$
1+\pi \tilde{t}_{c}+\ln \left(\pi \tilde{t}_{c}\right)=\ln \left(\frac{1}{2 \pi \tilde{\epsilon}}\right)
$$

where we now have

$$
\begin{aligned}
\tilde{t}_{c} & =\frac{t_{c}\left(\frac{m}{k} U\right)}{\lambda}=\frac{t_{c} m U}{2 \pi} \\
\tilde{\epsilon} & =\frac{\epsilon}{\lambda}=\frac{\epsilon}{2 \pi} \sqrt{m^{2}+n^{2}} \\
\lambda & =\frac{2 \pi}{k}=\frac{2 \pi}{\sqrt{m^{2}+n^{2}}}
\end{aligned}
$$

The singularity forms along the lines $m x+n y=b$, parallel to the generator of the $3 \mathrm{D}$ cylindrical sheet, where $b$ is some constant. The constant $c$ takes on an infinite number of values, corresponding to the regions of locally accumulated vorticity. Eq. 11.6, Eq. 11.9 and Eq. 11.18 - Eq. 11.21 summarize the main results of this chapter. They show that the singularity which forms following a finite amplitude, 3D normal mode perturbation to an undisturbed vortex sheet is essentially the same as Moore's result for the $2 \mathrm{D}$ perturbation. When $\epsilon, k$ and $U$ are held constant, it is clear that $t_{c}$ is a minimum for the $2 \mathrm{D}$ case $n=0$. 


\section{Chapter 12 Discussion}

A means for computing the motion of regularized vortex sheets in three dimensions is presented, utilizing automatic mesh refinement to maintain an accurate surface representation despite significant deformation. Smooth $\left(C^{1}\right)$ surface representation via interpolation over triangular patches increases the order of the overall computation. Three surface types (SWP0, 1, and 2) are demonstrated, suitable for modeling vortex rings, vortex jet/tubes and planar shear layers. The methods described here can be used to model several physical flows such as vortex ring interaction, trailing wing tip vortex pair interaction, shocked density interface motion, jet and shear layer development and stirring.

The computational technique presented is $O\left(N^{3 / 2}\right)$ in theory, but unfortunately reverts to $O\left(N^{2}\right)$ when the triangle length scales become non-uniform. A full hierarchical spatial subdivision technique is required to achieve faster theoretical speeds. Nonetheless, computations run 15-20 times faster than a straightforward implementation of the spatial integration required in Eq. 2.22. The automatic mesh generation and accompanying $C^{1}$ surface interpolants performed quite well, contributing efficiency and accuracy. The parametric surface representation was a success for all geometries studied here, but is a limiting factor if surfaces with inherently more complicated geometries are desired.

With the local hardware used at the time of this writing, computations were CPU limited at approximately $10^{5}$ to $2 \cdot 10^{5}$ triangles. Memory, either RAM or on disk, were never in critical demand.

Several simulations were performed, most notably a perturbed planar shear layer. Long time evolution revealed the onset of streamwise vorticity via variation of shear on the sheet surface with respect to spanwise position. The simulated surface evolution and delayed onset of spanwise vorticity was qualitatively similar to a previous experimental investigation of the shear layer [30]. The ratio of spanwise to streamwise 
circulation was found to lie between 7 and 9 for the duration of the simulation.

A new technique for estimating the critical time of a evolving vortex sheet was presented which is applicable with regularized vortex sheet simulations. The method was shown to work well with $2 \mathrm{D}$ vortex sheet motion, and agree with previous work.

The numerics lead us to examine in detail the motion of normal mode perturbations of the planar vortex sheet. We were able to show analytically that (i) the evolution of a vortex sheet from an initial condition corresponding to a finite amplitude 3D normal mode disturbance remains cylindrical for all time and (ii) in a rotated reference frame the non-linear evolution is the same as that for an equivalent $2 \mathrm{D}$ normal mode with suitably modified velocity jump and ratio of disturbance amplitude to wavelength. Thus the singularity time in a $3 \mathrm{D}$ vortex sheet perturbed as such is equivalent to the $2 \mathrm{D}$ normal mode singularity time and furthermore the singularity appears simultaneously along a line on the surface. The critical time is given by

$$
1+\pi \tilde{t}_{c}+\ln \left(\pi \tilde{t}_{c}\right)=\ln \left(\frac{1}{2 \pi \tilde{\epsilon}}\right)
$$

where

$$
\begin{aligned}
\tilde{t}_{c} & =\frac{t_{c}\left(\frac{m}{k} U\right)}{\lambda}=\frac{t_{c} m U}{2 \pi} \\
\tilde{\epsilon} & =\frac{\epsilon}{\lambda}=\frac{\epsilon}{2 \pi} \sqrt{m^{2}+n^{2}} \\
\lambda & =\frac{2 \pi}{k}=\frac{2 \pi}{\sqrt{m^{2}+n^{2}}} .
\end{aligned}
$$

\subsection{Directions for Future Work}

The spirit of this project may be continued in many ways. From a computational standpoint, the program is not far from a complete parallelized $O(N)$ implementation. This state would allow for upwards of $10^{6}$ triangles to be present in any one mesh. Also, the periodic influence of SWP1 surfaces should be incorporated into the code. It would be impressive to drop the necessity of surface parameterization, for this would allow computations on simple sphere-like surfaces as well as complicated, multiply- 
connected surfaces. Perhaps more light could be shed on the spiraling growth of length scales as $\sigma$ tends to zero with a fully 3D investigation.

Many different fluid characteristics can be examined with regularized vortex sheets. It would be interesting to see how random perturbations develop for all the different surface geometries. Also, there is a class of flows in which shear layers grow from separation points, that vortex sheets may model quite well. Lastly, the dynamics, including the core, of vortex rings could be examined with several concentric SWP0 surfaces working in concert. 


\section{Bibliography}

[1] M. Abramowitz and I. E. Stegun. Handbook of Mathematical Functions. Dover, New York, 1972.

[2] M. E. Agishtein and A. A. Migdal. Dynamics of vortex surfaces in three dimensions: Theory and simulations. Physica D, 40:91-118, 1989.

[3] C. Anderson. A vortex method for flows with slight density variations. J. Comp. Phys., 61:417-444, 1985.

[4] W. T. Ashurst and E. Meiburg. Three-dimensional shear layers via vortex dynamics. J. Fluid Mech., 189:87-116, 1988.

[5] G. Baker and M. J. Shelley. On the connection between thin vortex layers and vortex sheets. J. Fluid Mech., 215:161-194, 1990.

[6] G. R. Baker, D. I. Meiron, and S. A. Orszag. Boundary integral methods for axisymmetric and three-dimensional Rayleigh-Taylor instability problems. Physica $D, 12: 19-31,1984$.

[7] C. L. Berman and L. Greengard. A renormalization method for the evaluation of lattice sums. J. Math. Phys., 35(11):6036-6048, 1994.

[8] G. Birkhoff and J. Fisher. Do vortex sheets roll up? Rend. Circ. Mat. Palermo, $8: 77,1959$.

[9] E. Boender. Reliable delaunay-based mesh generation and mesh improvement. Comm. Num. Meth. Eng., 10:773-783, 1994.

[10] R. E. Caflisch. Mathematical analysis of vortex dynamics. In R. E. Caflisch, editor, Mathematical Aspects of Vortex Dynamics, pages 1-24, 1988. 
[11] J. C. Cavendish. Automatic triangulation of arbitrary planar domains for the finite element method. Int. J. Num. Meth. Eng., 8:679-696, 1974.

[12] A. J. Chorin and P. S. Bernard. Discretization of a vortex sheet, with an example of roll-up. J. Comp. Phys., 13:423-429, 1973.

[13] S. J. Cowley, G. R. Baker, and S. Tanveer. On the formation of Moore curvature singularities in vortex sheets. J. Fluid Mech., 378:233-267, 1999.

[14] G. R. Cowper. Gaussian quadrature formulas for triangles. Int. J. Num. Meth. Eng., 7:405-408, 1973.

[15] N. Didden. On the formation of vortex rings: Rolling-up and production of circulation. J. App. Math. and Phys., 30:101-116, 1979.

[16] W. H. Frey and D. A. Field. Mesh relaxation: A new technique for improving triangulations. Int. J. Num. Meth. Eng., 31:1121-1131, 1991.

[17] P. R. Garabedian. Partial Differential Equations. Wiley, New York, 1964.

[18] T. N. T. Goodman and H. B. Said. A $C^{1}$ triangular interpolant suitable for scattered data interpolation. Comm. App. Num. Meth., 7:479-485, 1991.

[19] L. F. Greengard. The Rapid Evaluation of Potential Fields in Particle Systems. MIT Press, Cambridge, 1988.

[20] E. Harabetian, S. Osher, and C. Shu. An Eulerian approach for vortex motion using a level set regularization procedure. J. Comp. Phys., 127:15-26, 1996.

[21] J. F. Hass and B. Sturtevant. Interaction of weak shock waves with cylindrical and spherical gas inhomogeneities. J. Fluid Mech., 181:41-76, 1987.

[22] T. Y. Hou, J. S. Lowengrub, and M. J. Shelley. The long-time motion of vortex sheets with surface tension. Phys. Fluids, 9:1933-1954, 1997.

[23] T. Ishihara and Y. Kaneda. Singularity formation in three-dimensional motion of a vortex sheet. J. Fluid Mech., 300:339-366, 1995. 
[24] T. Ishihara and Y. Kaneda. Singularity formation in the shape of a vortex sheet in three dimensions - numerical sumulation. Proc. 2nd Inter. Work. on Vort. Flows and Rel. Num. Meth., pages 463-479, 1996.

[25] Y. Kaneda. A representation of the motion of a vortex sheet in a threedimensional flow. Phys. Fluids A, 2(3):458-461, 1990.

[26] O. M. Knio and A. F. Ghoniem. Three-dimensional vortex simulation of roll-up and entrainment in a shear layer. J. Comp. Phys., 97:172-223, 1991.

[27] R. Krasny. Desingularization of periodic vortex sheet roll-up. J. Comp. Phys., 65:292-313, 1986.

[28] R. Krasny. A study of singularity formation in a vortex sheet by the point-vortex approximation. J. Fluid Mech., 167:65-93, 1986.

[29] R. Krasny. Vortex sheet computations: Roll-up, wakes, separation. Proc. AMSSAIM Summer Seminar on App. Math., 1990.

[30] J. C. Lasheras and H. Choi. Three-dimensional instability of a plane free shear layer: an experimental study of the formation and evolution of streamwise vortices. J. Fluid Mech., 189:53-86, 1988.

[31] A. Leonard. Computing three-dimensional incompressible flows with vortex elements. Ann. Rev. Fluid Mech., 17:523-559, 1985.

[32] K. Lindsay. A Three-Dimensional Cartesian Tree-Code and Applications to Vortex Sheet Roll-Up. PhD thesis, The University of Michigan, 1997.

[33] S. H. Lo. A new mesh generation scheme for arbitrary planar domains. Int. J. Num. Meth. Eng., 21:1403-1426, 1985.

[34] E. Meiburg. Three-dimensional vortex dynamics simulations. In S. I. Green, editor, Fluid Vortices, pages 651-679, 1995. 
[35] D. I. Meiron, G. R. Baker, and S. A. Orszag. Analytical structure of vortex sheet dynamics. Part 1. Kelvin-Helmholtz instability. J. Fluid Mech., 114:283$298,1982$.

[36] J. W. Miles. On the disturbed motion of a plane vortex sheet. J. Fluid Mech., $3: 538,1958$.

[37] D. W. Moore. The equation of motion of a vortex layer of small thickness. Stud. Appl. Math., 58:119-140, 1978.

[38] D. W. Moore. The spontaneous appearance of a singularity in the shape of an evolving vortex sheet. Proc. Roy. Soc. London A, 365:105-119, 1979.

[39] D. W. Moore and D. I. Pullin. Inviscid separated flow over a non-slender delta wing. J. Fluid Mech., 305:307-345, 1995.

[40] Q. Nie and G. Baker. Application of adaptive quadrature to axi-symmetric vortex sheet motion. J. Comp. Phys., 143:49-69, 1998.

[41] M. Nitsche. Evolution of a cylindrical and a spherical vortex sheet. Proc. 2nd Inter. Work. on Vort. Flows and Rel. Num. Meth., pages 401-414, 1996.

[42] M. Nitsche and R. Krasny. A numerical study of vortex ring formation at the edge of a circular tube. J. Fluid Mech., 276:139-161, 1994.

[43] J. Peraire, M. Vahdati, K. Morgan, and O. C. Zienkiewicz. Adaptive remeshing for compressible flow computations. J. Comp. Phys., 72:467-485, 1987.

[44] D. I. Pullin. The large-scale structure of unsteady self-similar rolled-up vortex sheets. J. Fluid Mech., 88:401-430, 1978.

[45] D. I. Pullin. Numerical studies of surface-tension effects in non-linear KelvinHelmholtz and Rayleigh-Taylor instability. J. Fluid Mech., 119:507-532, 1982.

[46] D. I. Pullin and W. R. C. Phillips. On a generalization of kaden's problem. $J$. Fluid Mech., 104:45-53, 1981. 
[47] D. I. Pullin and P. G. Saffman. Vortex dynamics in turbulence. Ann. Rev. Fluid Mech., 30:31-51, 1998.

[48] L. Rosenhead. The spread of vorticity in the wake behind a cylinder. Proc. Roy. Soc. London A, 127:590-612, 1930.

[49] L. Rosenhead. The formation of vortices from a surface of discontinuity. Proc. Roy. Soc. London A, 134:170-192, 1931.

[50] J. W. Rottman and P. K. Stansby. On the ' $\delta$-equations' for vortex sheet evolution. J. Fluid Mech., 247:527-549, 1991.

[51] P. G. Saffman. Vortex Dynamics. Cambridge University Press, Cambridge, 1992.

[52] P. G. Saffman and G. R. Baker. Vortex interactions. Ann. Rev. Fluid Mech., 11: 95-122, 1979.

[53] J. K. Salmon and M. S. Warren. Skeletons from the treecode closet. J. Comp. Phys., 111:136-155, 1994.

[54] R. Samtaney and D. I. Pullin. On initial and self-similar solutions of the compressible Euler equations. Phys. Fluids, 8:2650-2655, 1996.

[55] M. J. Shelley. A study of singularity formation in vortex-sheet motion by a spectrally accurate vortex method. J. Fluid Mech., 244:493-526, 1992.

[56] G. Tryggvason. Simulation of vortex sheet roll-up by vortex methods. J. Comp. Phys., 80:1-16, 1989.

[57] G. Tryggvason, W. J. A. Dahm, and K. Sbeih. Fine structure of vortex sheet roll-up by viscous and inviscid simulation. Trans. ASME I: J. Fluids Eng., 113: 31-36, 1991.

[58] A. Vincent and M. Meneguzzi. The dynamics of vorticity tubes in homogeneous turbulence. J. Fluid Mech., 258:245-254, 1994. 
[59] T. J. Willmore. An Introduction to Differential Geometry. Oxford University Press, London, 1959.

[60] G. S. Winckelmans and A. Leonard. Contributions to vortex particle methods for the computation of three-dimensional incompressible unsteady flows. J. Comp. Phys., 109:247-273, 1993.

[61] H. Yamada and T. Matsui. Preliminary study of mutual slip-through of a pair of vortices. Phys. Fluids, 21:292-94, 1978. 


\section{Appendix A Notation}

A Surface area of the vortex sheet

$\alpha \quad$ model coefficient of curvature behavior near $t_{c}$

$B \quad$ Number of multi-pole boxes needed to encase sheet surface

$\beta \quad$ model coefficient of curvature behavior near $t_{c}$

$D \quad$ Distance between physical space locations $\boldsymbol{x}$ and $\boldsymbol{x}^{\prime}$, or $\mathbf{X}$ and $\mathbf{X}^{\prime}$

$D_{\sigma} \quad$ Smoothed distance between physical space locations $\boldsymbol{x}$ and $\boldsymbol{x}^{\prime}$, or $\mathbf{X}$ and $\mathbf{X}^{\prime}$

$\delta \quad$ Dirac delta function, subscript represents true vortex sheet variables

$d X \quad$ Length of segment in physical space corresponding to segment with components $(d \xi, d \eta)$ in parameter space

$E, F, G \quad$ First fundamental coefficients of the mapping $\mathbf{X}$

$E_{T O L} \quad$ Error tolerance for box-target velocity evaluation

$\epsilon \quad$ Perturbation magnitude; strain on a triangle edge

$\Gamma \quad$ Circulation

$\boldsymbol{\gamma} \quad$ Vortex sheet strength

$\eta \quad 2^{\text {nd }}$ parameter (Lagrangian) of parameter space

$J \quad$ Jacobian of the mapping function $\mathbf{X}(\xi, \eta)$

$K \quad$ Gaussian curvature of the surface

K Biot-Savart kernel

$\kappa_{a}, \kappa_{b} \quad$ Principal curvatures of the surface $\mathbf{X}$

$\kappa_{T} \quad$ Total curvature

$L, M, N$ Second fundamental coefficients of the mapping $\mathbf{X}$

$L_{B} \quad$ Side length of box containing triangles for multi-pole expansion 
$M^{i} \quad$ First, second and third moments of vorticity

$\mu \quad$ Mean curvature of the surface

$N \quad$ Number of triangles in the surface

n Unit normal to the surface $\mathbf{X}(\xi, \eta)$

$\boldsymbol{\omega}, \boldsymbol{\omega}_{\delta} \quad$ Vorticity field

$P_{0} \quad$ Base period of a periodic domain

$P_{1} \quad$ Those periodic extensions of $P_{0}$ adjacent to $P_{0}$

$P_{1 \infty} \quad$ All periods of a periodic domain except $P_{0}$

$P_{2} \quad$ Those periodic extensions of $P_{0}$ adjacent to $P_{1}$

$P_{2 \infty} \quad$ All periods of a periodic domain except $P_{0} \cup P_{1}$

$\tilde{P}_{i} \quad$ Lattice of centroids of the periods included in $P_{i}$

$\phi^{+}, \phi^{-} \quad$ Velocity potential functions above and below the surface

$R \quad$ Resolution, as a triangle density scaling parameter

$S \quad$ Physical space surface of vortex sheet

$S_{i, j} \quad$ Doubly infinite summations required to evaluate influence of images in SWP2 sheets

$\Sigma \quad$ Parameter space surface of vortex sheet

$s_{1}, s_{2} \quad$ Scaling factors of $\tilde{\Sigma}$ axes

$\sigma$

Biot-Savart kernel smoothing parameter, subscript represents smoothed vortex sheet variables

SWP0,1,2 Surface with periodicity of 0,1 or 2

$T \quad$ Average number of triangles in a multi-pole expansion box

$t_{c} \quad$ Critical time of vortex sheet evolution (curvature singularity)

$\theta_{1}, \theta_{2} \quad$ Angles of $\tilde{\Sigma}$ axes

$\mathbf{u}, \mathbf{u}_{\delta} \quad$ Velocity field

$\mathbf{u}_{\sigma} \quad$ Smooth velocity field, resulting from smoothed Biot-Savart kernel

$\mathbf{u}^{V} \quad$ Velocity contribution (influence) from vorticity contained in physical space region $V$ 
$V \quad$ Volume in physical space: contains a group of triangles; a single period; all of physical space

W Parameterized vortex sheet strength

$X \quad x$ component of the mapping function $\mathbf{X}$

$\mathbf{X}, \mathbf{X}(\xi, \eta)$ Mapping from parameter to physical space

$\boldsymbol{x} \quad$ Field point in physical space

$\xi \quad 1^{\text {st }}$ parameter (Lagrangian) of parameter space

$\zeta_{1}, \zeta_{2}, \zeta_{3} \quad$ Barycentric coordinates 


\section{Appendix B Invariants}

Proof that the integral quantities presented in $\S 2.5$ are time invariant (for regularized vortex sheet flows) is demonstrated by first introducing new surface parameters $(r, s)$ such that $r$ is perpendicular and $s$ is parallel to vortex lines. By construction, we have $\Gamma_{s}=0$ and

$$
\mathbf{W} d \xi d \eta=\left(\Gamma_{\xi} \mathbf{X}_{\eta}-\Gamma_{\eta} \mathbf{X}_{\xi}\right) d \xi d \eta=\Gamma_{r} \mathbf{X}_{s} d r d s
$$

The total vorticity is thus

$$
\iint_{\xi} \mathbf{W} d \xi d \eta=\int_{r} \Gamma_{r}\left\{\int_{s} \mathbf{X}_{s} d s\right\} d r=\mathbf{0},
$$

since vortex lines are closed on the surface (we consider here the surface type SWP0 only). The velocity field becomes

$$
\dot{\mathbf{X}}(r, s)=\frac{1}{4 \pi} \iint_{r^{\prime}} \int_{s^{\prime}} \Gamma_{r^{\prime}} \mathbf{X}_{s^{\prime}} \times \mathbf{K} d r^{\prime} d s^{\prime}
$$

where $\mathbf{K}=\left(\mathbf{X}-\mathbf{X}^{\prime}\right) / D_{\sigma}^{3}$ and $D_{\sigma}=\left[\left|\mathbf{X}-\mathbf{X}^{\prime}\right|^{2}+\sigma^{2}\right]^{1 / 2}$. For the first moment of vorticity, we have

$$
\mathbf{I}=\frac{1}{2} \iint_{\xi} \mathbf{X} \times \mathbf{W} d \xi d \eta=\frac{1}{2} \iint_{r} \Gamma_{\boldsymbol{s}} \mathbf{X} \times \mathbf{X}_{s} d r d s .
$$

Time differentiation and integration by parts yields

$$
\dot{\mathbf{I}}=\iint_{r} \Gamma_{r} \dot{\mathbf{X}} \times \mathbf{X}_{s} d r d s
$$


Substitution of the velocity field (Eq. B.3) yields

$$
\dot{\mathbf{I}}=\frac{1}{4 \pi} \iiint_{r} \int_{s} \int_{r^{\prime}} \Gamma_{r} \Gamma_{r^{\prime}}\left(\mathbf{X}_{s^{\prime}} \times \mathbf{K}\right) \times \mathbf{X}_{s} d r d s d r^{\prime} d s^{\prime} .
$$

Expanding the vector triple product, dropping the subsequent $\left(\mathbf{X}_{s} \cdot \mathbf{X}_{s^{\prime}}\right) \mathbf{K}$ term due to asymmetry (i.e., $\mathbf{K}$ changes sign when the prime and un-prime coordinates swap positions, while the dot product does not), and utilizing the identity $\frac{\partial}{\partial s} D_{\sigma}^{-1}=-\mathbf{X}_{s} \cdot \mathbf{K}$ gives

$$
\dot{\mathbf{I}}=-\frac{1}{4 \pi} \iint_{r} \int_{r^{\prime}} \Gamma_{s^{\prime}} \Gamma_{r^{\prime}}\left\{\int_{s} \frac{\partial}{\partial s} D_{\sigma}^{-1} d s\right\} \mathbf{X}_{s^{\prime}} d r d r^{\prime} d s^{\prime}=\mathbf{0} .
$$

Analogous arguments apply for the proof of $\dot{\mathbf{A}}=0$. Volume is preserved if (a) the bounding surface moves with the fluid, and (b) the velocity field is incompressible. The former is implied in Eq. 2.22 and Eq. B.3; the latter is apparent from an alternate form of Eq. 2.22:

$$
\mathbf{u}_{\sigma}(\boldsymbol{x})=\frac{1}{4 \pi} \nabla_{\boldsymbol{x}} \times \int_{\Sigma} \frac{\mathbf{W}^{\prime}}{\left[\left|\boldsymbol{x}-\mathbf{X}^{\prime}\right|^{2}+\sigma^{2}\right]^{1 / 2}} d \Sigma^{\prime}
$$

With the new parameters, kinetic energy becomes

$$
T=\frac{1}{8 \pi} \iiint_{r} \int_{r^{\prime}} \Gamma_{s^{\prime}} \Gamma_{r} \Gamma_{r^{\prime}} \frac{\mathbf{X}_{s} \cdot \mathbf{X}_{s^{\prime}}}{D} d r d s d r^{\prime} d s^{\prime}
$$

Time differentiation, symmetry arguments, and utilization of

$$
\frac{\partial}{\partial s}\left[D_{\sigma}^{-1}\left(\dot{\mathbf{X}} \cdot \mathbf{X}_{s^{\prime}}\right)\right]=-D_{\sigma}^{-3}\left(\mathbf{X}-\mathbf{X}^{\prime}\right) \cdot \mathbf{X}_{s}\left(\dot{\mathbf{X}} \cdot \mathbf{X}_{s^{\prime}}\right)+D_{\sigma}^{-1}\left(\dot{\mathbf{X}} \cdot \mathbf{X}_{s^{\prime}}\right)
$$


for integration by parts lead to

$$
\begin{aligned}
\dot{T}=\frac{1}{4 \pi} \iiint_{r} \int_{r^{\prime}} \int_{s^{\prime}} \Gamma_{r} \Gamma_{r^{\prime}} D^{-3}\{ & {\left[\left(\mathbf{X}-\mathbf{X}^{\prime}\right) \cdot \mathbf{X}_{s}\right]\left(\dot{\mathbf{X}} \cdot \mathbf{X}_{s^{\prime}}\right)-} \\
& {\left.\left[\left(\mathbf{X}-\mathbf{X}^{\prime}\right) \cdot \dot{\mathbf{X}}\right]\left(\mathbf{X}_{s} \cdot \mathbf{X}_{s^{\prime}}\right)\right\} d r d s d r^{\prime} d s^{\prime} }
\end{aligned}
$$

Substitution of the velocity field Eq. B.3 and some manipulation with vector identities finally gives

$$
\begin{aligned}
\dot{T}= & \frac{1}{16 \pi^{2}} \iiint_{r} \int_{r^{\prime}} \int_{s^{\prime}} \int_{r^{\prime \prime}} \int_{s^{\prime \prime}} \Gamma_{r} \Gamma_{r^{\prime}} \Gamma_{r^{\prime \prime}} \mathbf{X}_{s} . \\
& \frac{\left[\left(\mathbf{X}-\mathbf{X}^{\prime}\right) \times \mathbf{X}_{s^{\prime}}\right] \times\left[\left(\mathbf{X}-\mathbf{X}^{\prime \prime}\right) \times \mathbf{X}_{s^{\prime}}\right]}{\left[\left|\mathbf{X}-\mathbf{X}^{\prime}\right|^{2}+\sigma^{2}\right]^{3 / 2}\left[\left|\mathbf{X}-\mathbf{X}^{\prime \prime}\right|^{2}+\sigma^{2}\right]^{3 / 2}} d r d s d r^{\prime} d s^{\prime} d r^{\prime \prime} d s^{\prime \prime}
\end{aligned}
$$

which vanishes due to the asymmetry in $\mathbf{X}^{\prime}$ and $\mathbf{X}^{\prime \prime}$. 


\section{Appendix C Surface Interpolant Details}

The function $X$ is defined local to a triangle with barycentric coordinates as $X=X\left(\zeta_{1}, \zeta_{2}, \zeta_{3}\right)$. Recall the coordinates are redundant with $\zeta_{1}+\zeta_{2}+\zeta_{3}=1$. However, aside from obtaining function values, we need also the first derivatives with respect to the parameter space variables, $X_{\xi}$ and $X_{\eta}$. We have

$$
\xi=\zeta_{1} \xi_{1}+\zeta_{2} \xi_{2}+\zeta_{3} \xi_{c}=\zeta_{1}\left(\xi_{1}-\xi_{3}\right)+\zeta_{2}\left(\xi_{2}-\xi_{3}\right)+\xi_{3}
$$

and a similar expression for $\eta$ which trivially lead to the Jacobian

$$
\frac{\partial(\xi, \eta)}{\partial\left(\zeta_{1}, \zeta_{2}\right)}=\left(\begin{array}{cc}
\xi_{1}-\xi_{3} & \eta_{1}-\eta_{3} \\
\xi_{2}-\xi_{3} & \eta_{2}-\eta_{3}
\end{array}\right)
$$

as depicted in Fig. C.1. Inverting the above matrix gives the necessary derivative

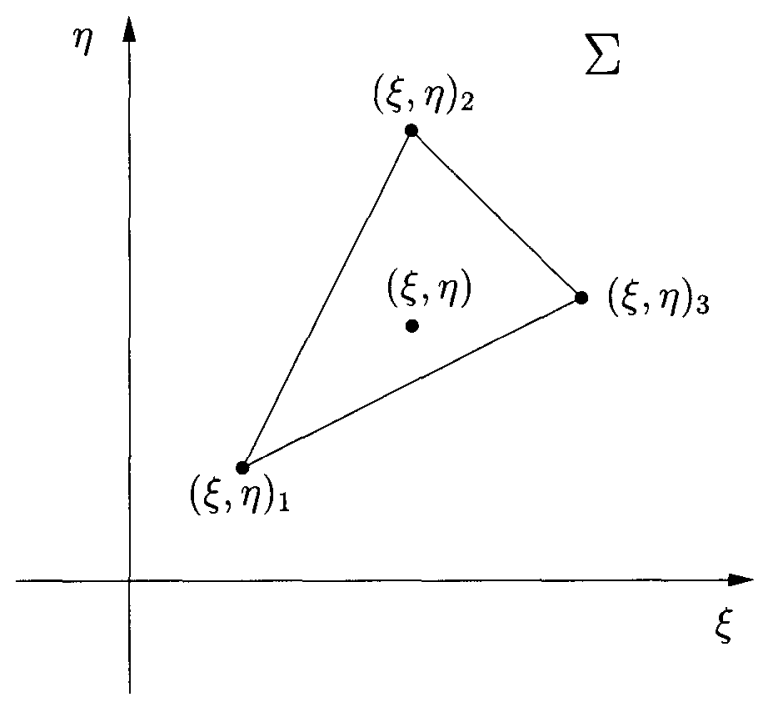

Figure C.1: Typical triangle in parameter space $(\Sigma)$. 
terms to evaluate

$$
\begin{aligned}
X_{\xi} & =\frac{\partial X}{\partial \zeta_{1}} \frac{\partial \zeta_{1}}{\partial \xi}+\frac{\partial X}{\partial \zeta_{2}} \frac{\partial \zeta_{2}}{\partial \xi}+\frac{\partial X}{\partial \zeta_{3}} \frac{\partial \zeta_{3}}{\partial \xi} \\
& =\frac{\partial \zeta_{1}}{\partial \xi}\left(\frac{\partial X}{\partial \zeta_{1}}-\frac{\partial X}{\partial \zeta_{3}}\right)+\frac{\partial \zeta_{2}}{\partial \xi}\left(\frac{\partial X}{\partial \zeta_{2}}-\frac{\partial X}{\partial \zeta_{3}}\right) \\
X_{\eta} & =\frac{\partial X}{\partial \zeta_{1}} \frac{\partial \zeta_{1}}{\partial \eta}+\frac{\partial X}{\partial \zeta_{2}} \frac{\partial \zeta_{2}}{\partial \eta}+\frac{\partial X}{\partial \zeta_{3}} \frac{\partial \zeta_{3}}{\partial \eta} \\
& =\frac{\partial \zeta_{1}}{\partial \eta}\left(\frac{\partial X}{\partial \zeta_{1}}-\frac{\partial X}{\partial \zeta_{3}}\right)+\frac{\partial \zeta_{2}}{\partial \eta}\left(\frac{\partial X}{\partial \zeta_{2}}-\frac{\partial X}{\partial \zeta_{3}}\right)
\end{aligned}
$$

The derivatives of $X$ with respect to the barycentric coordinates $\zeta_{i}$ are obtained from differentiation of Eq. 3.2. 


\section{Appendix D Regularizing the 2D, Periodic}

\section{Kernel}

It is interesting to note that when considering $2 \mathrm{D}$ periodic vortex sheets with a smooth kernel (i.e., length scale cut-off added as in Eq. 2.22), one can take two different approaches in deriving the evolution equation. Either the periodic extension of the exact (non-smoothed) kernel is derived first and the new periodic kernel smoothed, or the operations are done in the reverse order. The differences are detailed below.

The exact (non-smoothed) evolution equations for a $2 \mathrm{D}$ vortex sheet are

$$
\begin{aligned}
& \frac{\partial X}{\partial t}(\Gamma, t)=-\frac{1}{2 \pi} \int_{\text {sheet }} \frac{\hat{y}}{\hat{x}^{2}+\hat{y}^{2}} d \tilde{\Gamma}, \\
& \frac{\partial Y}{\partial t}(\Gamma, t)=\frac{1}{2 \pi} \int_{\text {sheet }} \frac{\hat{x}}{\hat{x}^{2}+\hat{y}^{2}} d \tilde{\Gamma},
\end{aligned}
$$

where $\hat{x}=X(\Gamma, t)-X(\tilde{\Gamma}, t), \hat{y}=Y(\Gamma, t)-Y(\tilde{\Gamma}, t)$, and $(X, Y)$ denote the sheet position. Eq. D.1 is simply the Birkhoff-Rott equation, without the common complex notation. If we first assume the sheet is periodic in $\Gamma$ (with unit period), then smooth the kernel by the addition of $\sigma^{2}$, the evolution equations become

$$
\begin{aligned}
& \frac{\partial X}{\partial t}(\Gamma, t)=-\frac{1}{2} \int_{0}^{1} \frac{\sinh 2 \pi \hat{y}}{\cosh 2 \pi \hat{y}-\cos 2 \pi \hat{x}+\sigma^{2}} d \tilde{\Gamma}, \\
& \frac{\partial Y}{\partial t}(\Gamma, t)=\frac{1}{2} \int_{0}^{1} \frac{\sin 2 \pi \hat{x}}{\cosh 2 \pi \hat{y}-\cos 2 \pi \hat{x}+\sigma^{2}} d \tilde{\Gamma} .
\end{aligned}
$$

These are the evolution equations used by Krasny [27].

But if the operations are done in the reverse order, i.e., the kernel is smoothed 
first and then the sheet is assumed periodic in $\Gamma$, we obtain

$$
\begin{aligned}
& \frac{\partial X}{\partial t}(\Gamma, t)=-\frac{1}{2} \int_{0}^{1} \frac{\sinh 2 \pi y_{\sigma}}{\cosh 2 \pi y_{\sigma}-\cos 2 \pi \hat{x}} \frac{\hat{y}}{y_{\sigma}} d \tilde{\Gamma} \\
& \frac{\partial Y}{\partial t}(\Gamma, t)=\frac{1}{2} \int_{0}^{1} \frac{\sin 2 \pi \hat{x}}{\cosh 2 \pi y_{\sigma}-\cos 2 \pi \hat{x}} d \tilde{\Gamma}
\end{aligned}
$$

where $y_{\sigma}=\sqrt{\hat{y}^{2}+\sigma^{2}}$. In this thesis it is appropriate to use Eq. D.3 as it is consistent with the smooth 3D Biot-Savart velocity field (Eq. 2.22). 


\section{Appendix E Evaluation of the Double}

\section{Periodic Summations}

As described in $§ 6.3$ several summations are needed to evaluate the velocity influence of the double periodic extension, $P_{2 \infty}$. In detail, we have

$$
S_{i, j}=2 \sum_{m=2}^{\infty}\left[T_{m, 0}^{i, j}+T_{0, m}^{i, j}+2 T_{m, m}^{i, j}\right]+4 \sum_{m=2}^{\infty} \sum_{n=1}^{m-1}\left[T_{m, n}^{i, j}+T_{n, m}^{i, j}\right]
$$

where the individual terms $T_{m, n}^{i, j}$ depends on $(i, j)$ as depicted in Tab. 6.1. For unit periodic wavelength in physical space we have

$$
x=m, \quad y=n, \quad D_{\sigma}=\sqrt{m^{2}+n^{2}+\sigma^{2}},
$$

so that in combination with Tab. 6.1 the terms $T_{m, n}^{i, j}$ are specified. The double summations must be calculated numerically for each desired smoothing parameter $\sigma$. However, they only need be evaluated once.

For example, with $\sigma=1 / 10$ and $i=j=1$, we have

$$
T_{m, n}^{1,1}=\frac{m^{2}}{\left(m^{2}+n^{2}+1 / 100\right)^{5 / 2}}
$$

which is substituted into Eq. E.1 to produce Tab. E.1. These numeric values are determined via a series extrapolation technique such as Levin's u-transform. 


\begin{tabular}{l|ll|l}
\hline$S_{0,0}$ & 3.61367386064313 & $S_{1,1}$ & 1.80492983701292 \\
$S_{0,1}$ & 0.381418661728640 & $S_{1,2}$ & 0.190364032377155 \\
$S_{0,2}$ & 0.0690596974330716 & $S_{1,3}$ & 0.0344581056623391 \\
$S_{0,3}$ & 0.0143486108393474 & $S_{1,4}$ & 0.00715850938870520 \\
& & & \\
$S_{2,3}$ & 0.0375092324585367 & $S_{3,3}$ & 0.152510218861995 \\
$S_{2,4}$ & 0.00581893175435931 & $S_{3,4}$ & 0.0285675888140927 \\
\hline
\end{tabular}

Table E.1: Summation values $S_{i, j}$ for $\sigma=1 / 10$. 